\title{
Energetic particle influence on the Earth's atmosphere
}

Article

Accepted Version

Mironova, I. A., Aplin, K. L., Arnold, F., Bazilevskaya, G. A., Harrison, R. G., Krivolutsky, A. A., Nicoll, K. A., Rozanov, E. V., Turunen, E. and Usoskin, I. (2015) Energetic particle influence on the Earth's atmosphere. Space Science Reviews, 194 (1-4). pp. 1-96. ISSN 0038-6308 doi:

https://doi.org/10.1007/s11214-015-0185-4 Available at https://centaur.reading.ac.uk/40966/

It is advisable to refer to the publisher's version if you intend to cite from the work. See Guidance on citing.

To link to this article DOI: http://dx.doi.org/10.1007/s11214-015-0185-4

Publisher: Springer

All outputs in CentAUR are protected by Intellectual Property Rights law, including copyright law. Copyright and IPR is retained by the creators or other copyright holders. Terms and conditions for use of this material are defined in the End User Agreement.

www.reading.ac.uk/centaur 
Central Archive at the University of Reading

Reading's research outputs online 


\title{
Energetic particle influence on the Earth's atmosphere
}

\author{
Irina A. Mironova - Karen L. Aplin · Frank \\ Arnold • Galina A. Bazilevskaya • R. Giles \\ Harrison - Alexei A. Krivolutsky - Keri A. \\ Nicoll • Eugene V. Rozanov · Esa Turunen · \\ Ilya G. Usoskin
}

Received: date / Accepted: date

\author{
I. A. Mironova \\ Earth Physics Department, Institute and Faculty of Physics, St.Petersburg State University, \\ Ulyanovskaya 1, 198504, St.Petersburg, Russia \\ Tel.: +78124284634 \\ E-mail: i.a.mironova@spbu.ru \\ K. L. Aplin \\ Physics Department, University of Oxford, Denys Wilkinson Building, Keble Road, Oxford, \\ OX1 3RH, United Kingdom \\ Tel.:+441865273491 \\ E-mail: k.aplin1@physics.ox.ac.uk \\ F. Arnold \\ Atmospheric Physics Division, Max-Planck-Institute for Nuclear Physics P.O.Box 103980, D \\ 69129 Heidelberg, Germany \\ Tel.:+496221516 \\ emailfrank.arnold@mpi-hd.mpg.de \\ G. A. Bazilevskaya \\ Lebedev Physics Institute, Russian Academy of Science, Leninskiy pr.53, Moscow, Russia \\ Tel.:+74954854263 \\ E-mail: gbaz@rambler.ru \\ R. G. Harrison \\ Department of Meteorology, University of Reading, P.O Box 243, Earley Gate, Reading Berks \\ RG6 6BB, United Kingdom \\ Tel.:+441189316690 \\ E-mail: r.g.harrison@reading.ac.uk \\ A. A. Krivolutsky \\ Laboratory for Atmospheric Chemistry and Dynamics, Central Aerological Observatory Rus- \\ sian Federal Service for Hydrometeorology and Enviromental monitoring, Pervomayskaya str. \\ 3, 141700 Dolgoprudny, Moscow, Russia \\ Tel.: +74954087706 \\ E-mail: alexei.krivolutsky@rambler.ru \\ K. A. Nicoll \\ Department of Meteorology, University of Reading, P.O Box 243, Earley Gate, Reading Berks \\ RG6 6BB, United Kingdom \\ Tel.: +441189316690 \\ E-mail: k.a.nicoll@reading.ac.uk \\ E. V. Rozanov \\ PMOD/WRC and IAC ETHZ, Dorfstrasse 33, CH-7260 Davos Dorf, Switzerland
}


Abstract This manuscript gives an up-to-date and comprehensive overview of the effects of energetic particle precipitation (EPP) onto the whole atmosphere, from the lower thermosphere/mesosphere through the stratosphere and troposphere, to the surface. The paper summarizes the different sources and energies of particles, principally galactic cosmic rays (GCRs), solar energetic particles (SEPs) and energetic electron precipitation (EEP). All the proposed mechanisms by which EPP can affect the atmosphere are discussed, including chemical changes in the upper atmosphere and lower thermosphere, chemistry-dynamics feedbacks, the global electric circuit and cloud formation. The role of energetic particles in Earth's atmosphere is a multi-disciplinary problem that requires expertise from a range of scientific backgrounds. To assist with this synergy, summary tables are provided, which are intended to evaluate the level of current knowledge of the effects of energetic particles on processes in the entire atmosphere.

Keywords Energetic Particle Precipitation (EPP), Galactic Cosmic ray (GCR), Solar Energetic Particle (SEP), Energetic Electron Precipitation (EEP), Ions, Column of the Earth Atmosphere, Atmospheric Processes, Atmospheric Chemistry, Global Electric Circuit, Aerosols and Clouds, Climate

Tel $:+41814175135$

E-mail: e.rozanov@pmodwrc.ch

E. Turunen

Sodankylä Geophysical Observatory, Tähteläntie 62, FIN-99600 Sodankylä, Finland

Tel.:+358-294-480813

E-mail: et@sgo.fi

I.G. Usoskin

Sodankylä Geophysical Observatory (Oulu unit) and ReSoLVE Centre of Excellence University of Oulu, FIN-90014 Oulu, Finland

Tel.:+35885531377

E-mail: ilya.usoskin@oulu.fi 


\section{Contents}

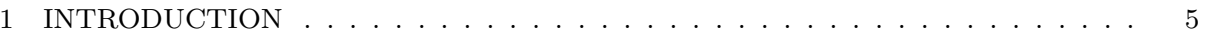

2 ENERGETIC PARTICLES . . . . . . . . . . . . . . . . . . . . . . 7

2.1 Sources of protons in the Earth atmosphere . . . . . . . . . . . . . 7

2.1.1 Cosmic rays in the near Earth space and atmosphere . . . . . . . . . 8

2.1.2 Monitoring of energetic particles with neutron monitors, balloon-borne and satellite-borne detectors . . . . . . . . . . . . . . . . . . . . . . . 12

Ground-based neutron monitors . . . . . . . . . . . . . . . . . . . . . . 12

Balloon-borne monitoring . . . . . . . . . . . . . . . . . . . . 13

Satellite-borne monitoring . . . . . . . . . . . . . . . . . . . . . . . . . . 15

2.1.3 Galactic cosmic ray variability . . . . . . . . . . . . . . . . . 16

11- and 22-year modulation . . . . . . . . . . . . . . . . . . . . . . . . . 16

Forbush decreases and 27-day recurrent variations . . . . . . . . . . . . . 18

2.1.4 Solar energetic particles . . . . . . . . . . . . . . . . . . . 21

2.1.5 Modelling energetic particles and the cosmic ray induced ionization . . . 26

Analytical model . . . . . . . . . . . . . . . . . . . . . . 26

Monte-Carlo models . . . . . . . . . . . . . . . . . . . . . . . . 28

Comparison of different approaches . . . . . . . . . . . . . . . . . . . . . . 30

2.2 Sources of the electrons in the Earth's atmosphere . . . . . . . . . . . . . 33

Radiation belt electrons . . . . . . . . . . . . . . . . . . . . . . 34

Auroral electrons . . . . . . . . . . . . . . . . . . . . . . . . 35

2.2.1 Types of precipitation . . . . . . . . . . . . . . . . . . . . . . . . . . . . . . . . . . . . . . . . . 36

2.2.2 Recent measurements in the atmosphere . . . . . . . . . . . . . . . . . . 39

3 ENERGETIC PARTICLES INFLUENCE ON ATMOSPHERIC PROCESSES . . . 43

3.1 Ions . . . . . . . . . . . . . . . . . 43

3.1.1 Introduction to atmospheric ions . . . . . . . . . . . . . . 43

3.1.2 Sources and sinks of atmospheric electrically charged species . . . . . . 44

3.1.3 Ion composition: vertical structure and observations . . . . . . . . . 46

3.1.4 Ion induced aerosol formation . . . . . . . . . . . . . . . . . 49

3.1.5 Laboratory investigations of ion induced nucleation . . . . . . . . . . . . 52

3.1.6 Model simulations of atmospheric ion induced nucleation . . . . . . . . 53

3.1.7 Growth of critical molecular clusters to CCN size . . . . . . . . . . . . . 53

3.2 Effects of energetic particles on neutral gas-phase chemistry and the

ozone layer . . . . . . . . . . . . . . . . . . . . . . . . . . . 54 54

3.2.1 Introduction to EPPs effect on chemistry of the atmosphere . . . . . . . 54

3.2.2 The processes involved . . . . . . . . . . . . . . . . 54

Direct chemical effects . . . . . . . . . . . . . . . . . . . . . . 55

Indirect effects . . . . . . . . . . . . . . . . . . . . . . . . . . . . . . 57

3.2.3 Modeling and observations of ozone changes caused by EPP . . . . . . 58

Auroral electrons . . . . . . . . . . . . . . . . . . . . . 58

Radiation belt electrons . . . . . . . . . . . . . . . . . . . . 61

Solar protons . . . . . . . . . . . . . . . . . . . . . . 64

Galactic cosmic rays . . . . . . . . . . . . . . . . . . . . . 70

3.3 Atmospheric electricity . . . . . . . . . . . . . . . . . . . 72

3.3.1 Introduction to the global electric circuit . . . . . . . . . . . . . . . 72

3.3.2 Global electric circuit variability . . . . . . . . . . . . . . . . . . . . . 74

3.3.3 Effect of energetic particles on the global electric circuit . . . . . . . . . 75

Periodic variations . . . . . . . . . . . . . . . . . . . . . . 75

Transient energetic particle effects . . . . . . . . . . . . . . . . . 77

3.3.4 Surface measurements . . . . . . . . . . . . . . . . . . . . . . . . . . . . . . . . . . . 77

3.3.5 Airborne measurements . . . . . . . . . . . . . . . . . . . . 79

3.3.6 GCR and lightning ...................... . . 81

3.4 Clouds ............................ 83

3.4.1 Mesospheric clouds . . . . . . . . . . . . . . . . . 83

3.4 .2 Stratospheric clouds . . . . . . . . . . . . . . . . . . 84

3.4 .3 Troposphere ........................ 85

Clear-air effect . . . . . . . . . . . . . . . . . . . 85

Near-cloud effect . . . . . . . . . . . . . . . . . . . . . . . . . . . . . . . . . 86

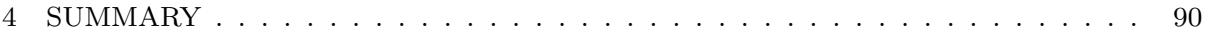


Definitions used in the SUMMARY Tables 2-7. . . . . . . . . . . . . . . . . 91

Abbreviations used in the manuscript . . . . . . . . . . . . . . . . . . . . . . . . 98

Author contributions . . . . . . . . . . 99

Acknowledgements ......................... . . . . . 99

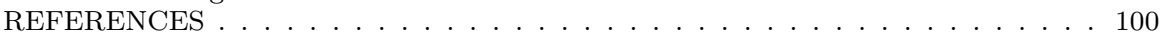

\section{List of Tables}

1 Magnitudes (in \%) of the 11-year modulation cycle as recorded by different cosmic ray detectors. . . . . . . . . . . . . . . . 18

2 SUMMARY Table. Assessment of lower thermosphere and mesosphere atmospheric responses to energetic particles and cosmic rays with possible climate relevance. . . . . . . . . . . . . . . 92

3 SUMMARY Table. Assessment of stratosphere atmospheric responses to energetic particles and cosmic rays with possible climate relevance. . 93

4 SUMMARY Table. Assessment of troposphere atmospheric responses to energetic particles and cosmic rays with possible climate relevance. . . . 94

5 SUMMARY Table. Assessment of clouds response to energetic particles and cosmic rays with possible climate relevance.Beginning. . . . . . . . 95

6 SUMMARY Table. Assessment of clouds response to energetic particles and cosmic rays with possible climate relevance. Continue. . . . . . . . 96

$7 \quad$ SUMMARY Table. Assessment of clouds response to energetic particles and cosmic rays with possible climate relevance.Continue. . . . . . . . . 97 


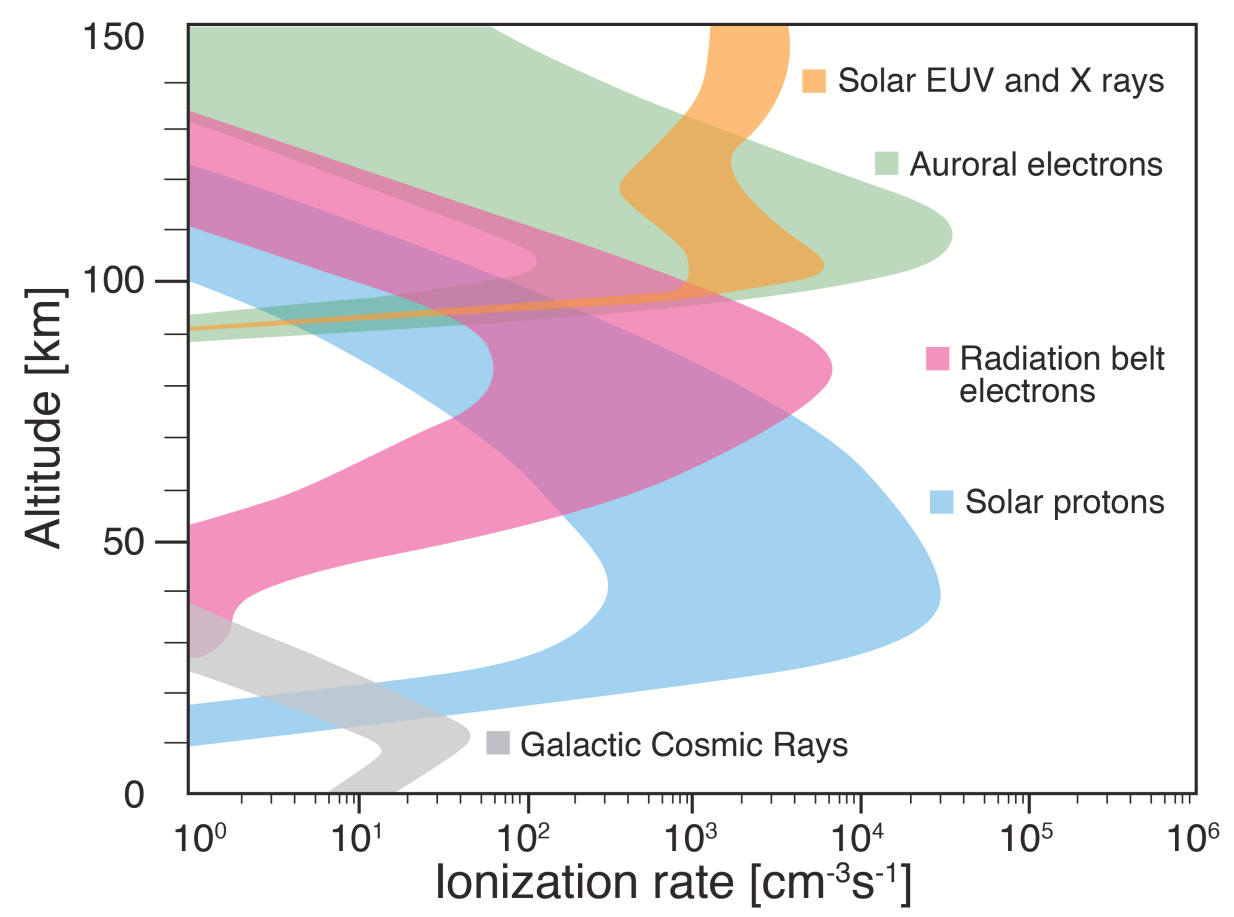

Fig. 1 Instantaneous ionization rates of EPP, Solar EUV and X-ray of the Earth's atmospheric layers. The figure is modified after Baker et al. (2012).

\section{INTRODUCTION}

Many published papers review the potential impact of energetic particle precipitation (EPP) on atmospheric chemistry and temperature (Rozanov et al. 2012; Sinnhuber et al. 2012; Krivolutsky and Repnev 2012), on global electric circuit (Rycroft et al. 2012) and cloud formation (Enghoff and Svensmark 2008; Laken et al. 2012), and dynamics from the thermosphere down to the surface. There are also a few reviews focusing on the results of concerted project activities, like, e.g., Seppälä et al. (2014). However these reviews usually present an overview of results for short-time projects period or an overview of only some aspects, for example the effects of different types of energetic particles are considered separately, as are the potential effects on the atmosphere, and possibly climate. In spite of all of these reviews, a summary of all these aspects together is still missing from the literature.

A schematic of the various types of energetic particle precipitation into Earth's atmosphere, as well as the altitude profile of the ionization they create is given in Figure 1. Here one can see that different types of energetic precipitation particles (EPPs) as well as solar EUV and X-rays are involved in the atmospheric ionization. Energetic particles (EPs) are one of the main sources of ionization below $100 \mathrm{~km}$, where solar EUV and X-rays emissions are strongly attenuated. Such EPPs can be Auroral and Radiation belt (Relativistic) Electrons, Solar (Solar Energetic Particles) and Galactic (Galactic Cosmic Rays) Protons, see Fig. 1. 
Significant improvements have been made recently in both modelling and observations of energetic particles (EPs), so that many links of the chain linking energetic particle precipitation to atmospheric processes are now better understood. Although the overall picture is still somewhat diffuse because of the many different aspects of atmospheric physics and chemistry concerned, the new level of understanding emerging gives a better perspective on the problem of energetic particle precipitation contribution to natural atmospheric variability. Investigation of the role energetic particles play in the processes affecting Earth's atmosphere is a multi-disciplinary problem that needs to be solved across several subjects, extending solar and cosmic ray physics to atmospheric physics/chemistry and dynamics. The potential role of energetic particles still remains poorly known and is often considered based on qualitative or, at best, first principles arguments and correlations. In particular, the IPCC Fifth Assessment Report (IPCC 2013), only considers the changes in the total solar irradiance and does not take into consideration changes at different wavelengths as well as the energetic particle precipitation (EPP) impact on the Earth's atmosphere. This therefore unavoidably limits the understanding of natural climate variability. Numerous efforts have been taken to account for these processes, in particular those related to the impact of energetic particles on the polar middle atmosphere. However, large uncontrolled uncertainties in some EPP data sets still lead to uncertainties in our knowledge of the effects, which can be up to an order of magnitude, still leave some results inconclusive. The goal of the present review manuscript is to summarize the current knowledge of the effect of EPP in the Earth's atmosphere. The paper aims to provide a wide and complete up to date review of the state of the art on energetic particle properties, their transport in the atmosphere, and the physics and chemistry of the upper, middle and lower parts of the atmosphere. The manuscript is organized as follows: Section 2. ENERGETIC PARTICLES, which describes the different sources and energies, measurements and variability of EPP. Section 3. ENERGETIC PARTICLES INFLUENCE ON ATMOSPHERIC PROCESSES, which includes sections on EPP effects on (a) atmospheric ion properties (Section 3.1); (b) gas phase chemistry and the ozone layer (Section 3.2); (c) atmospheric electricity and the global electrical circuit (Section 3.3); (d) cloud effects. Section 4. SUMMARY summarises the findings of the paper in terms of summary tables, which attempt to convey the level of scientific understanding of various EPP processes as well as assess the importance of EPP in terms of their potential climate impacts. 


\section{ENERGETIC PARTICLES}

The Earth is continuously bombarded by energetic charged particles coming from outer space (Grieder 2001; Vainio et al. 2009), collectively known as energetic precipitating particles (EPPs) which penetrate into the Earth's atmosphere and can influence a variety of atmospheric processes (Bazilevskaya et al. 2008). The majority of energetic particles (EPs) originate from outer space and are known as cosmic rays (CRs), consisting mostly of protons. The solar wind provides an additional source of EPP that are less energetic and mostly electrons, which are accelerated/trapped in the Earth's magnetosphere. Such particles precipitate into the atmosphere from various regions in the magnetosphere, both driven by solar wind conditions and intrinsic magnetospheric processes. The multitude of processes and their dynamical variability leads to high variations in spatial, temporal, flux and energy distributions of the precipitating particles. In the polar cusp and the polar cap region loss-cone particles precipitate directly on open magnetic field lines, whereas at auroral ovals, and subauroral latitudes precipitation on closed field lines occurs when trapped particles are pushed to the losscone, which may happen even at midlatitudes for relativistic electron precipitation from radiation belts.

\subsection{Sources of protons in the Earth atmosphere}

Sources of protons in Earth's atmosphere are CRs that may be of galactic, solar and heliospheric origin. Galactic cosmic rays (GCRs) permanently arriving from outside of the solar system consist of the most energetic particles, conventionally with energies from $\approx 10^{7} \mathrm{eV}$ up to $\approx 10^{21} \mathrm{eV}\left(1 \mathrm{eV}=1.6 \cdot 10^{-19} \mathrm{~J}\right)$. Anomalous cosmic rays (ACRs) are accelerated inside the heliosphere, consist mainly of singly ionized atoms with energies below $100 \mathrm{MeV} /$ nuc (Cummings and Stone 1998) and do not play a role in atmospheric processes, because of their low abundance and low energy. Solar cosmic rays (SCRs) or solar energetic particles (SEPs) appear in near-Earth space sporadically, in connection with explosive energy releases at the Sun and may have energy up to $\approx 10 \mathrm{GeV}$. SEPs are generally accelerated on the Sun or in the inner heliosphere on interplanetary shocks. Auroral proton precipitation at subauroral latitudes and scattering of ring current protons provides a magnetospheric source of lower energy protons, which is of interest when considering upper atmospheric light emissions and the physics of proton transport processes, but due to the low energy is not discussed for the purpose of this review.

On the other hand, CRs form the main source of ionization in the atmosphere at altitudes above about $3 \mathrm{~km}$ and below about $60 \mathrm{~km}$. Although the degree of ionization is very small (the low-middle atmosphere is essentially neutral), the presence of a small amount of mobile ions is important for atmospheric chemistry and atmospheric electricity.

The flux of EPs is subject to solar modulation and varies with solar activity, each component having its own relation. While SEPs have higher fluxes and energy during the periods of active Sun, GCRs are inversely modulated by solar activity. Magnetospheric particle precipitation is subject to a variety of drivers and is found to be more frequent either during the maximum and a few years after maxima or during the decay and minimum phases of the solar cycle, depending on the occurence of coronal mass ejections and high speed solar wind streams. The latter is generally found to be more 
geoeffective in long term variations.

In this Section we give a phenomenological description of EPs flux variations, for different EPs components of the general totality of cosmic rays. Special emphasis is given to a relationship between the apparent EPs flux changes and different manifestations of solar activity. Although the sunspot number is commonly accepted as an index of solar activity dynamics, it should be noted that sunspots per se do not directly affect the GCR flux, whose modulation is a complex process of particle transport in the magnetized solar wind with frozen-in magnetic fields, inside the heliosphere. A detailed theoretical consideration of this process is beyond the scope of this paper and can be found elsewhere (Strauss et al. 2012; Potgieter 2013).

\subsubsection{Cosmic rays in the near Earth space and atmosphere}

The dominant component of CRs is made of protons, about $10 \%$ (in particle number) comprise He nuclei ( $\alpha$-particles), and other nuclei make up less than $1 \%$. However, due to their large number of nucleons and weaker modulation by heliospheric and geomagnetic fields, the relative contribution of heavier species to atmospheric ionization can be up to 30-50\% (Usoskin and Kovaltsov 2006). Electrons form a minor constituent of CRs (about $1 \%$ or less in the same energy range). Energy spectra of various components are, to the first order, similar to each other when the energy is given per nucleon (or electron, respectively). In more detail CR composition and energy spectra are considered in the works of Simpson (1983) and Gaisser (1990). Accordingly, we describe here mostly protons as the most abundant, and other species can be very roughly considered as their scaling.

The energy spectrum of extraterrestrial protons is schematically depicted in Fig. 2. The observed range of CR energy extends by nearly 15 orders of magnitude, from 1 $\mathrm{MeV}\left(1.6 \cdot 10^{-13} \mathrm{~J}\right)$, to an enormous value of $3 \cdot 10^{20} \mathrm{eV}(48 \mathrm{~J})$. The intensity of particles of these ultra-high energies which produce the extensive air showers (EAS) are small, amounting to less than $1 / \mathrm{km}^{2}$. sr. century (e.g., Stanev 2010).

The flux of extra-terrestrial cosmic rays depends on the level of solar activity and, to a lesser extent, conditions in the magnetosphere (Vainio et al. 2009). The flux of GCRs becomes lower during periods of high solar activity and vice-versa. However SEPs, existing only sporadically during periods of high solar activity, may occasionally enhance the flux of particles with energies tens-hundreds $\mathrm{MeV}$ by several orders of magnitude for hours-days, and in rare events called GLE (ground-level enhancement of cosmic rays) up to several $\mathrm{GeV}$, for minutes-hours. Magnetospheric particle precipitation is associated either with coronal mass ejections at high solar activity or with high-speed solar wind streams from coronal holes which is most frequent during the decaying phase and near the minimum of solar activity (Baker et al. 1993; Bazilevskaya and Svirzhevskaya 1998; Sinnhuber et al. 2012).

The Earth possess it's own magnetic field, which can, at a zero approximation, be considered as a dipole. This geomagnetic field deflects charged particles and acts as a spectrometer separating the arriving $C R$ particles according to their rigidity. The magnetic rigidity is defined as $R=c P / Z e$ (here $c$ is the speed of light, $P$ is the particle's momentum, $Z$ is the particle's charge number, and $e$ is the unit charge). In nuclear physics $e$ and $c$ are conventionally equal to 1 (Rossi 1940), so for protons $R$ is 


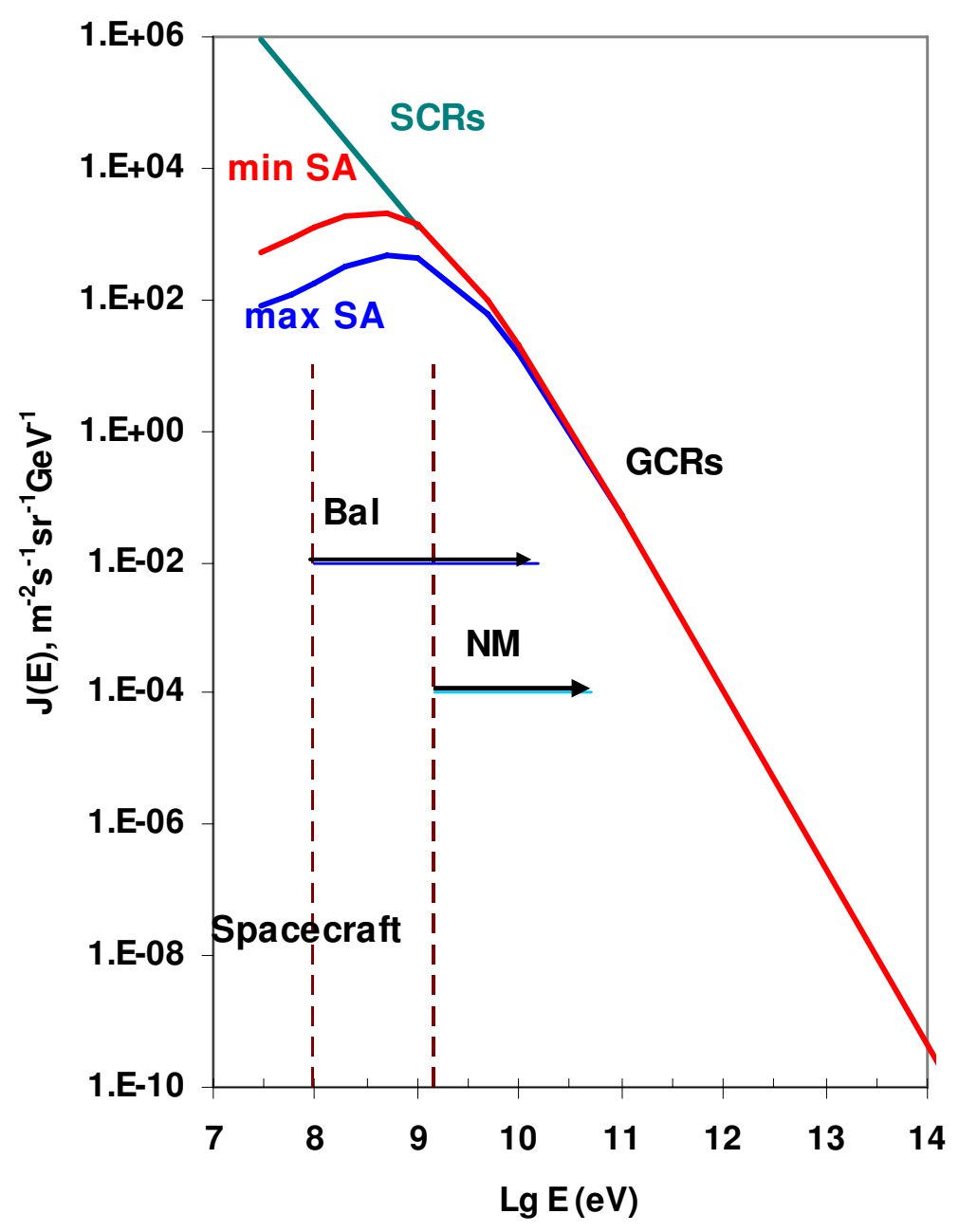

Fig. 2 Energy spectrum of protons in the near-Earth space. The GCR spectrum extends up to $n \cdot 10^{20} \mathrm{eV}$ (not shown here). A typical proton intensity for a medium-size SEP event (SCRs), of GCRs for a minimum and maximum of solar activity (SA) cycle are presented in the low-energy range. Also indicated are the domains of observations with spacecraft, balloons (Bal), and neutron monitors (NM).

numerically related to kinetic energy $T$.

$$
R=\sqrt{E^{2}+2 E_{o} T}
$$

where $E_{o}=0.938 \mathrm{GeV}$ is the proton's rest mass, $R$ is given in $\mathrm{GV}$ and $T$ in GeV. Rigidity is a key parameter for particle motion in magnetic fields. The particles with equal rigidities move in a similar way in a given magnetic field, even if their energies are different. Roughly speaking, the effect of geomagnetic shielding can be characterized by a cut-off rigidity, $R_{c}$, so that particles with lower rigidity cannot reach the given location. Although this is an oversimplification, it is proven to be a useful approach (Cooke et al. 1991; Smart et al. 2006), especially for ground -based observations (Nevalainen et al. 
2013). Most protected is the equatorial region, where charged particles must possess rigidity $R$ greater than $13-17 \mathrm{GV}$ to be able to reach the atmosphere, while there is no shielding in the polar region. We note that the geomagnetic field does not decelerate or terminate charged particles, it only bends their trajectories deflecting them away. A detailed review of geomagnetic shielding can be found elsewhere (e.g., Smart et al. 2000).

As well as the geomagnetic field, Earth's atmosphere also acts as a particle energy spectrometer, separating particles according to their energy. Primary CR particles with energies below several hundreds of $\mathrm{MeV} /$ nuc are simply stopped and absorbed in the atmosphere due to ionization losses. If, however, the energy of a primary particle is sufficiently high, it can collide with a nucleus of one of the atmospheric gases. This leads to a development of a nuclear-electromagnetic-muon cascade in the atmosphere (Particle Data Group 2004), which involves different species such as electrons, X-rays, muons, pions, kaons and nucleons. A schematic view of such a cascade is shown in Fig. 3. Due to development of the cascade (increasing the number of secondaries), the flux of ionizing particles first increases downwards in the atmosphere reaching maximum at the altitude of $17-27 \mathrm{~km}$ (depending on cut-off rigidity $R_{c}$ and the level of solar activity), but then decreases with altitude due to prevailing absorption. The effective energy of primary CRs is higher at lower latitudes and the cascades initiated by more energetic particles penetrate deeper into the atmosphere. Therefore the lower the altitude and latitude of the observational site, the higher the effective energy of primary CRs contributing to the flux of secondaries.

Atmospheric conditions also affect the observed fluxes of secondary CRs at a given location. The main atmospheric impacts on the secondary CR fluxes are known as barometric and temperature effects (Dorman 2004). Development of a cascade in the atmosphere depends on the air mass traversed by particles, which can be characterized by the barometric pressure at the location and the incident angle of the primary particle. Since the atmospheric pressure at a given location varies, the air mass above the observation level changes accordingly. The flux of particles $N_{\text {obs }}$ observed at pressure $P$ can be reduced to the flux $N_{\text {cor }}$ at the reference atmospheric pressure $P_{0}$ as $N_{\text {cor }}=N_{\text {obs }} \cdot \exp \left(\beta\left(P-P_{0}\right)\right)$, where $\beta$ is the so-called barometric coefficient with a typical value of $(7-8) \cdot 10^{-3} \mathrm{hPa}^{-1}$ for nucleonic component. This effect is significant for the nucleonic component of the secondary CRs (see Fig. 3) and is crucial for data obtained by neutron monitors. In order to properly account for the barometric effect, one needs to know only the easily measured barometric pressure, thus this correction is done straightforwardly. The temperature effect is much more difficult to correct for since it is related to the altitude distribution of the air mass, governed by the vertical profile of temperature. The effect is caused by production of very unstable particles such as $\pi$-, K-mesons, and most importantly muons, whose life-time is comparable with the time of flight in the atmosphere. The effect is two-fold. Higher temperatures cause air rarefaction (i.e. a reduction in air density), therefore mesons at higher altitude are more likely to decay rather than to suffer collision, thus increasing the number of generated muons (positive temperature effect). On the other hand, a muon produced at higher altitude has a higher probability to decay before reaching the detector (negative temperature effect). The net temperature effect depends on temperature distribution throughout the atmosphere and on the energy of the muons detected. Accordingly, in order to correct for the temperature effect one needs to know the temperature (or actually the air density) profile of the atmosphere above the observation site, which is not an easy measurement and requires additional instrumentation such as a lidar or mete- 


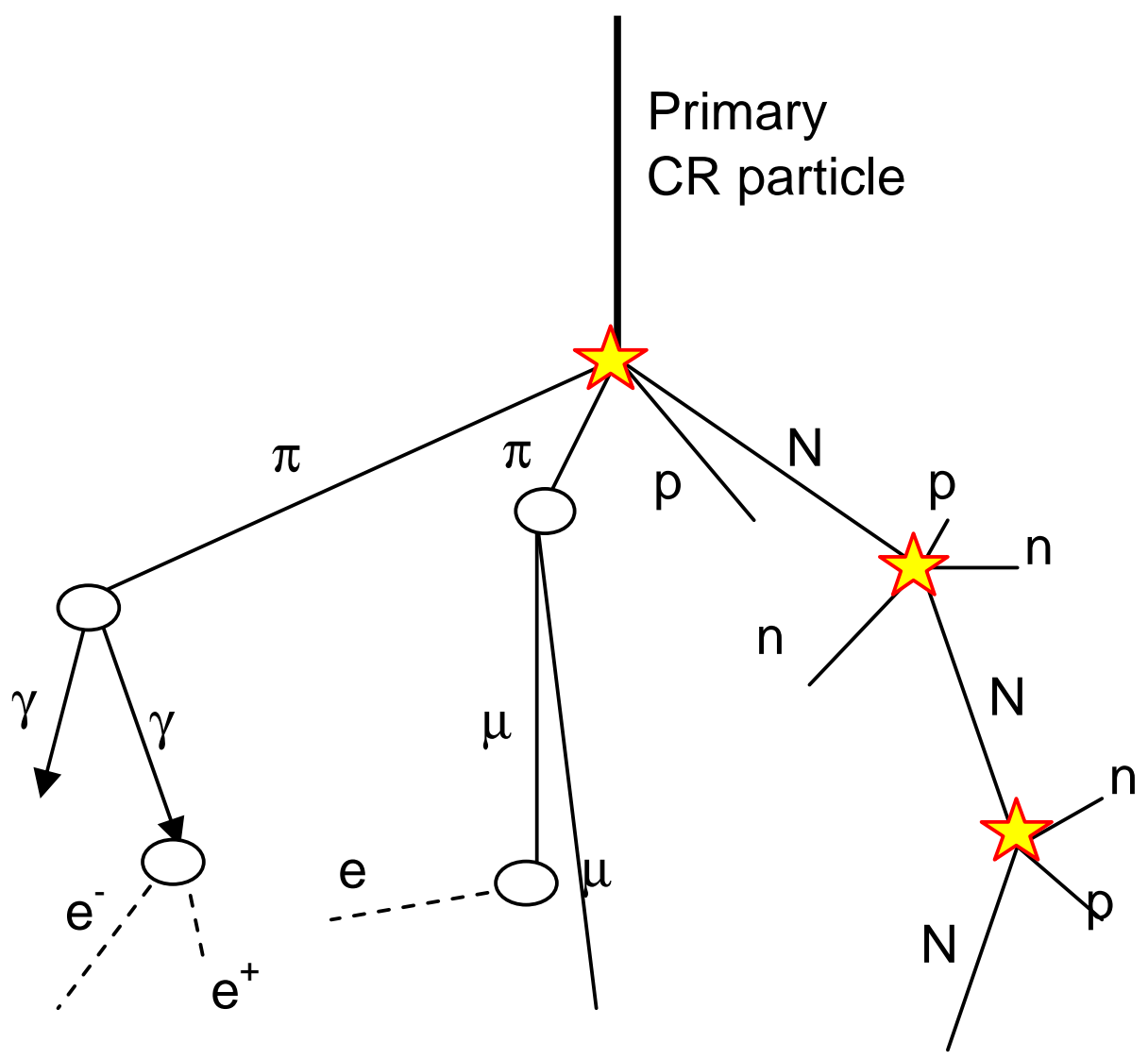

Fig. 3 Schematic view of an atmospheric cascade caused by energetic cosmic rays in the atmosphere. Left-to-right are denoted, respectively, the soft, muon and hadronic components of the cascade. Symbols "N, p, n, $\mu, \pi, \mathrm{e}^{ \pm}$and $\gamma$ " denote nuclei, protons, neutrons, muons, pions, electrons, positrons, and photons, respectively. Stars denote nuclear collisions, ovals decay processes. This sketch does not represent the full development of the cascade and serves solely as an illustration for the processes discussed in the text. After Usoskin (2011).

orological radiosonde. The temperature effect does not affect the nucleonic component of the cascade.

Thus, the CR intensity measured at Earth depends not only on the level of solar activity but also on the geomagnetic latitude and the altitude of the observational site. The low-energy fraction of CRs, which affects the upper polar atmosphere, can only be recorded at high latitudes and altitudes. This can be detected onboard spacecraft, rockets $(<100 \mathrm{MeV})$ and balloons $(>100 \mathrm{MeV})$. For the energy range above $1 \mathrm{GeV}$, the $\mathrm{CR}$ modulation is well measured by ground-based neutron monitors and muon telescopes. It should be kept in mind that the published records of the ground-based installations are typically corrected for the barometric and sometimes for the temperature effects in order to represent the primary $\mathrm{CR}$ variations, but the cosmic ray induced ionization (CRII) at a given location is influenced by the local pressure and temperature. 
2.1.2 Monitoring of energetic particles with neutron monitors, balloon-borne and satellite-borne detectors

It is important that a detector measuring variations of the CR flux provides sufficient statistical accuracy and that it is stable for many years of observations without essential uncontrolled instrumental drifts or other (e.g., seasonal) artifacts in the data. It is useful to measure the CR flux with standard instrumentation so that the data are comparable for different locations and times. The most extensive time series of homogeneous measurements of CR intensity is obtained with ground-based neutron monitors and balloon-borne detectors. Permanent monitoring of EPs and SEPs started with the Explorer 34 (IMP 4) mission in 1967, and is now continued by the GOES satellite series.

\section{Ground-based neutron monitors}

The concept of ground-based neutron monitors (NMs) was proposed by J. A. Simpson in 1948, as a standard instrument to measure cosmic ray intensities at Earth. He initiated the worldwide network of standard installations which has operated since the 1950s (Simpson 1948; Simpson et al. 1953; Hatton 1971). In total more than a hundred NM stations have participated in the neutron monitor network, with about 50 stations operating nowadays. A neutron monitor detects neutrons that are secondary products of the nucleonic branch of a cascade initiated in the atmosphere by primary cosmic rays with energies above roughly $1 \mathrm{GeV}$. Since the nucleonic cascade is attenuated with the amount of air overburden, the directly observed count rate needs to be corrected for the actual barometric pressure at the measurement site to be an index of the CR intensity. Typical time resolution of NM data is from 1 hour in the earlier years to seconds in modern instruments. Detail of the NM design and operations can be found elsewhere (Hatton 1971; Moraal et al. 2000). The NM is an energy-integrated device which measures all cosmic rays above the detection threshold. However, there is additional geomagnetic shielding (quantified in the geomagnetic cut-off rigidity $R_{\mathrm{c}}$ ) which varies over the globe from $R_{c}=0$ in polar regions (although the atmospheric shielding cut-off of $\approx 1 \mathrm{GV}$ is always present) to $R_{c}=13$ to $17 \mathrm{GV}$ in the equatorial regions. Therefore, by using data from the global NM network one can evaluate the spectrum of cosmic rays (Usoskin et al. 2005; Usoskin et al. 2011) as the effective energy of $\mathrm{CR}$ for a given NM depends on its location geomagnetic latitude (cut-off rigidity $\mathrm{R}_{c}$ ) and altitude, from about 10 to $40 \mathrm{GeV}$ (Alanko et al. 2003). Neutron monitors are often used for long term monitoring of CRs due to their reliable stability (Ahluwalia and Ygbuhay 2013).

Before NMs, there were long-term ground-based measurements performed by ionchambers (Forbush 1954, 1958; Chuprova et al. 2009) but they suffer from uncontrolled instrumental drifts and cannot be used for long-term studies without proper re-calibration (Forbush 1958; McCracken and Beer 2007; Ahluwalia 2011). Another common type of ground-based cosmic ray detector is the muon telescope, which measures the muon component of the cosmic ray induced atmospheric cascade. Because of the high penetration ability of muons, such detectors are often located at shallow underground depth to study higher energies of primary cosmic rays. The effective energy of muon detectors varies from $50-70 \mathrm{GeV}$ for a ground-based instrument to TeV energy for underground laboratories. Muon detectors should not be used to provide a stable basis for long-term studies of cosmic ray variations due to a number of complications 
which are difficult to account for: data are modulated not only by barometric pressure but also by the atmospheric density profile; there is no standard design for muon detectors; the scintillators commonly used in such detectors are known to be prone to uncontrolled aging.

\section{Balloon-borne monitoring}

Another way to measure the cosmic ray intensities on a regular basis is via the ion production rate in the atmosphere. Such measurements were conducted by H.V. Neher from 1930 up to the early 1970s (Neher 1967, 1971; Anderson 1973), using standard ionization chambers lifted by balloons. Each flight measured several hours of data. The chambers had $0.6 \mathrm{~mm}$ thick steel walls and were filled with air at $740 \mathrm{~mm} \mathrm{Hg}$ pressure. The temperature inside the chamber was kept to within $+/-10^{\circ} \mathrm{C}$ of $24^{\circ} \mathrm{C}$ during the balloon flights. The measurements of the ion production rate, $Q$ (ion pair $/ \mathrm{cm}^{3} / \mathrm{s} / \mathrm{atm}$ ), in the chamber with fixed pressure was then reduced to the ion production rate in situ (in the ambient atmosphere), $q$ (ion-pair $/ \mathrm{cm}^{3} / \mathrm{s}$ ). Although they produced some of the pioneering measurements of ionization in Earth's atmosphere, these measurements were not performed often enough to provide a robust data set for long term CR studies.

Regular monitoring of the charged particle fluxes (secondary CRs) in the atmosphere using balloon-borne detectors was initiated in the USSR by S.N. Vernov and A.N. Charakhchyan, during the International Geophysical Year in 1957 (Charakhchyan 1964; Bazilevskaya and Svirzhevskaya 1998; Stozhkov et al. 2009). This work continues to the present day under the supervision of Lebedev Physical Institute (hereafter LPI) in Moscow. Specially designed radiosondes equipped with Geiger counters are launched several times a week at several geographic sites including polar latitudes. The detectors are carefully calibrated in order to maintain the time series homogeneity. The device records the flux of protons with energies above several $\mathrm{MeV}$, electrons above hundreds $\mathrm{keV}$ and muons with energies above several $\mathrm{MeV}$ at atmospheric depths from the ground level up to $\approx 5 \mathrm{~g} / \mathrm{cm}^{2}$ (altitude $\approx 35 \mathrm{~km}$ ). While ascending in the atmosphere, the radiosonde records the secondary CR flux versus atmospheric depth, which is known as a transition curve with the Pfotzer maximum (Bazilevskaya et al. 2008). The measurements of the ion production rates by the Neher group and the LPI measurements of ionizing particle fluxes in the atmosphere overlapped during the 1960s, and can be related to each other. The upper panel of Fig. 4 shows the altitude dependence of the particle flux, $J$, and the ion pair production rate, $q$, at polar $\left(R_{c}=0 \mathrm{GV}\right)$, mid-latitude $\left(R_{c} \approx 2.5 \mathrm{GV}\right)$ and equatorial $\left(R_{c}>13 \mathrm{GV}\right)$ regions during a period of minimum solar activity. The in-situ ion production rate $q$ depends both on the flux of ionizing particles $J$ and the density of ambient air $n$, which leads to the shift of the maximum in the transition curves towards lower altitudes, as $q$ decreases with height because of the decreasing $n$. The ratio between $q$ and $J$ is shown in the lower panel of Fig. 4 (Bazilevskaya et al. 2000). While it strongly depends on the altitude, the relation to $R_{c}$ and solar activity is weak, and it can be fitted by a simple expression $q / J=A \cdot \exp (-B H)$, where $A=119.86 \mathrm{~cm}^{-1}, B=0.148$, and $H$ is the altitude in $\mathrm{km}$. This expression holds only for GCR and is not valid for SEP and electron precipitation.

The geomagnetic shielding (latitude) effect versus height is illustrated in Fig. 5 as the ratio of polar to equatorial values of $q$ and $J$ for the minimum and maximum of solar activity. This figure gives only a qualitative estimation, because there were no 


\section{CR flux AND ION PRODUCTION rate}
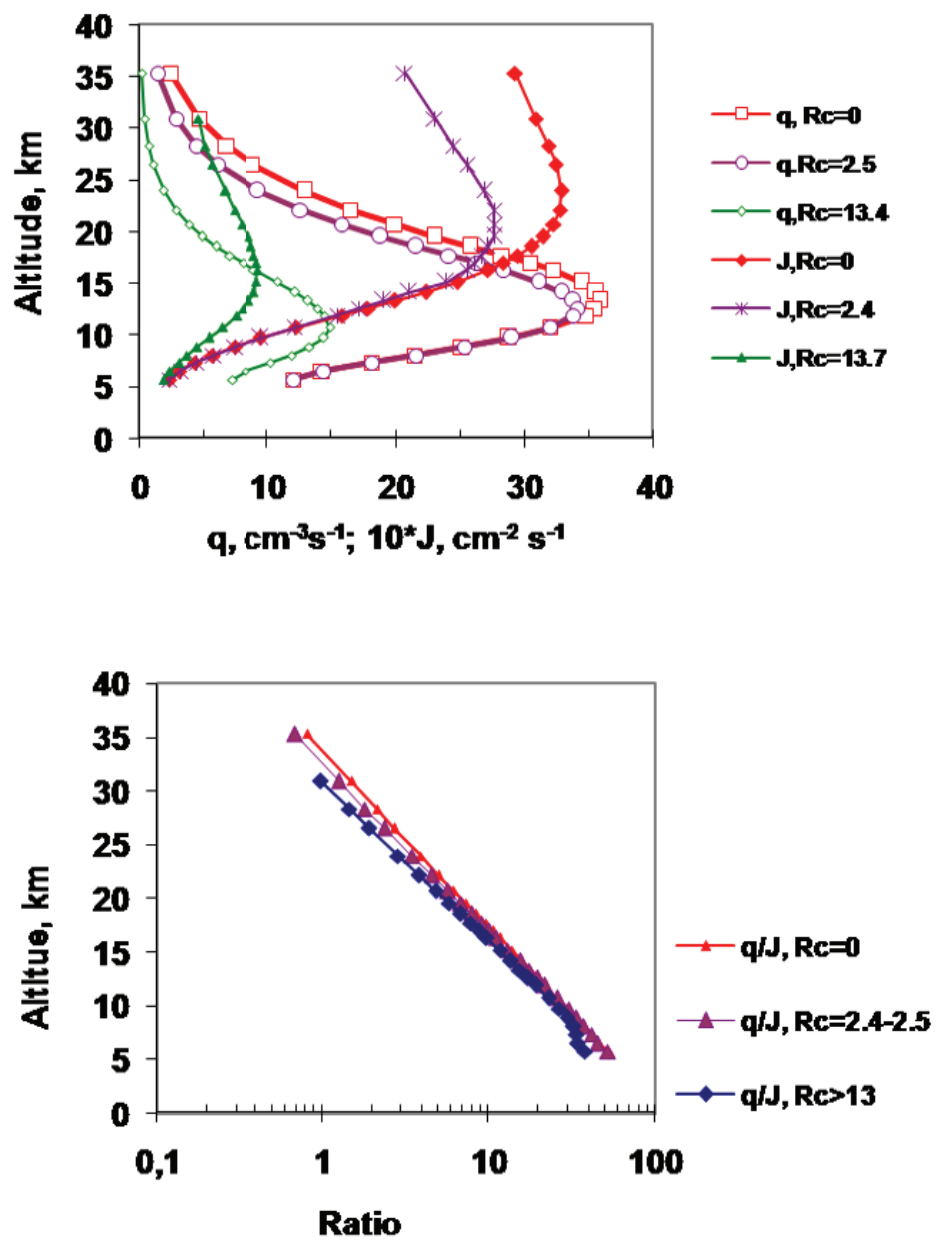

Fig. 4 Upper panel: transition curves in the atmosphere for the ion production rate $q$ (Neher 1971 ) and for the charged particle flux, $J$ (LPI observations) for polar and middle latitudes during solar minimum. Lower panel: ratio of $q / J$.

simultaneous observations at $R_{c}=0 \mathrm{GV}$ and $13 \mathrm{GV}$, and the constancy of the ionization/flux measured at equator is assumed. One can see that the $q\left(R_{c}=0\right) / q\left(R_{c}=13\right)$ ratio increases with altitude much stronger than $J\left(R_{c}=0\right) / J\left(R_{c}=13\right)$ for the particle flux. In general, the latitude effect is smaller during the maximum of solar activity, because of the reduction of low-energy GCRs. At sea level, according to neutron monitor observations (e.g., Moraal et al. 1989), the ratio of counting rates at $R_{c}=0 \mathrm{GV}$ to those at $R_{c}=13 \mathrm{GV}$ is 1.7 and 1.6 for the minimum and maximum of solar activity, 


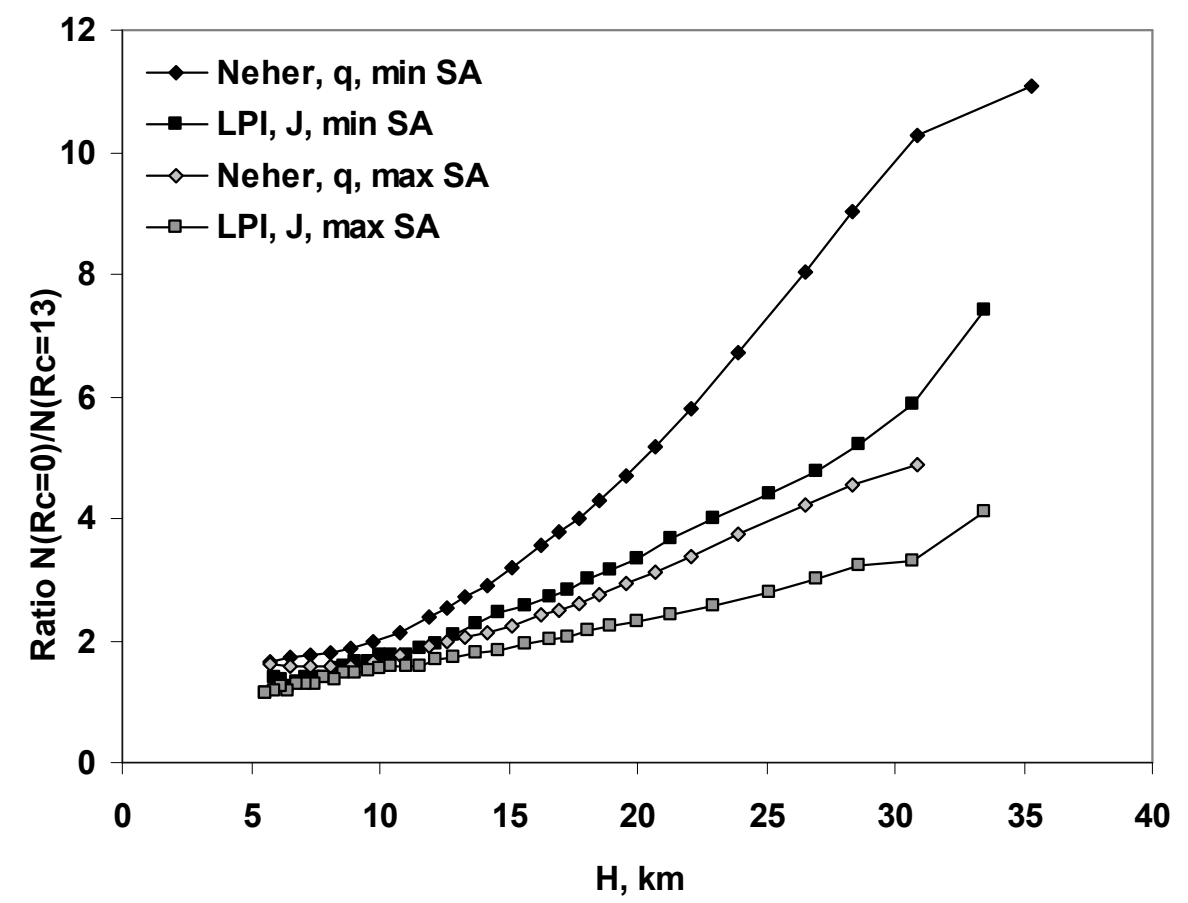

Fig. 5 Latitude effect vs. altitude in the atmosphere. Ratio of values at $R_{c}=0 \mathrm{GV}$ (pole) to those at $R_{c}=13 \mathrm{GV}$ (equator) for the ion production rate, $q$ (ion-pairs $/ \mathrm{cm}^{3} / \mathrm{s}$ ) and the charged particle flux, $J\left(\right.$ part $/ \mathrm{cm}^{2} / \mathrm{s}$ ) for the minimum and maximum of solar activity (SA). Data on ion production rate, labeled as "Neher" are taken from Neher (1971), data on charged particle fluxes, labeled as "LPI" are obtained at Lebedev Physical Institute. Reprinted from Stozhkov et al. (2009), with permission from Elsevier.

respectively.

\section{Satellite-borne monitoring}

Satellites are indispensable for SEP monitoring since the majority of SEPs have energy below $100 \mathrm{MeV}$ and do not penetrate into the atmosphere below $\approx 30 \mathrm{~km}$. However, the low-energy SEPs play a significant role in chemistry of the upper stratosphere and mesosphere (see Section 3) and should be taken into account in this context. Monitoring of SEPs have been performed routinely by the IMP (Interplanetary Monitoring Platform) Explorer satellites (1967-2006) and by the geostationary GOES (Geosynchronous Operational Environmental Satellite) satellites since 1986. Usually these satellites use different types of instrumentation, such as telescopes, spectrometers, magnetometers and charged particle detectors. In the framework of the 8th IMP mission, detection of EP was made by a telescope which measures fluxes of ions in four energy channels ranging from $0.05-0.20 \mathrm{MeV}$ to $2.1-4.5 \mathrm{MeV}$, and electrons in the ranges 30-90 and 
100-200 keV. The SEP telescope measures protons and alpha particles in the $0.5-1.8$ $\mathrm{MeV} / \mathrm{n}$ range. Each of the GOES satellites carries a suite of three detectors assembled aboard: a solid state telescope, a set of large-aperture, so-called DOME detectors, and a Cerenkov/solid-state-telescope called the HEPAD (High Energy Proton and Alpha Detector), which monitors the energetic particle population at geostationary orbit. These detectors measure the flux of energetic protons from $600 \mathrm{keV}$ to greater than $700 \mathrm{MeV}$ in 11 discrete channels as well as monitoring the electron and alpha particle populations.

There is virtually no geomagnetic shielding for SEPs at the height of $\approx 36000 \mathrm{~km}$ (the geostationary orbit). The data on solar protons and helium with energies 1-500 $\mathrm{MeV} /$ nuc are available in several energy channels with the time resolution of 1 and 5 minutes [http://goes.ngdc.noaa.gov/data/avg, http://spidr.ngdc.noaa.gov/spidr/]. Several SEP event catalogues have been made with information about the SEP energy spectra and intensity-time profiles which cover the period from 1955 onwards (see Vainio et al. 2009). It should be noted that satellite-borne detectors are usually not used for GCR monitoring because they are not suitable for measurements of fluxes of high-energy particles, except during dedicated missions such as IMP8, PAMELA or AMS (Adriani et al. 2013; Aguilar et al. 2013). The SEP fluxes should be derived from the satellite observations with proper account for the background count rates in each energy channel.

\subsubsection{Galactic cosmic ray variability}

\section{1- and 22-year modulation}

The galactic cosmic ray flux is modulated in the heliosphere by solar magnetic activity on different time scales. The dominant feature is the approximate 11-year cyclic variation which is in opposite phase to solar activity. Fig. 6 depicts monthly data from several neutron monitors and the balloon-borne detectors from LPI, compared with sunspot numbers. A strong anti-correlation between particle fluxes and solar activity is obvious, but the relation is not one-to-one but rather hysteresis-like, with $\mathrm{CR}$ being delayed against sunspot numbers depending on the polarity of the global solar magnetic field (Usoskin et al. 1998). The shape of maxima of the 11-year cycle in CR intensity (viz. solar minima) is peak-like for the $A$ - epochs (negative magnetic field in the northern hemisphere of the Sun) (1965s, 1987s, 2009s) and plateau-like for $A+$ epochs (1970s and 1990s), as defined by the drift effects of CRs on the heliospheric current sheet (HCS) (Jokipii and Kóta 1995). This feature does not imply a true 22-year cycle in CR modulation as is often naively assumed, as this effect becomes important only around solar minima when the HCS is relatively flat. The magnitude of the 11-year modulation cycle in CRs varies between a few and $60 \%$ depending on the effective energy range of the detector, which is in turn defined by the type of measured secondaries (ionizing particles, muons, nucleons), geomagnetic latitude $\left(R_{c}\right)$ and the altitude of the observation, as summarized in Table 1. Average magnitudes of the 11-year cycle in CRs were taken as $\left\langle J_{\max }\right\rangle-\left\langle J_{\min }\right\rangle$, where $\left\langle J_{\max }\right\rangle$ is the averaged value of a given instrument count rates (in \% of 1965) for Jul-Sep.1965, Nov.1976-Jan.1977, Jan-Mar.1987, May-Jul.1996, and Jul-Sep.2009 for cycles 19-23 respectively. $\left\langle J_{\min }\right\rangle$ is averaged for Nov.1958-Jan.1959, Apr-Jun.1970, Nov.1982-Jan.1983, Feb-Apr.1990, and Oct-Dec.2000. One can also see in Fig. 6 that the CR flux for the recent solar minimum 2009-2010 is 5-10\% higher than the previous solar minima in accordance 

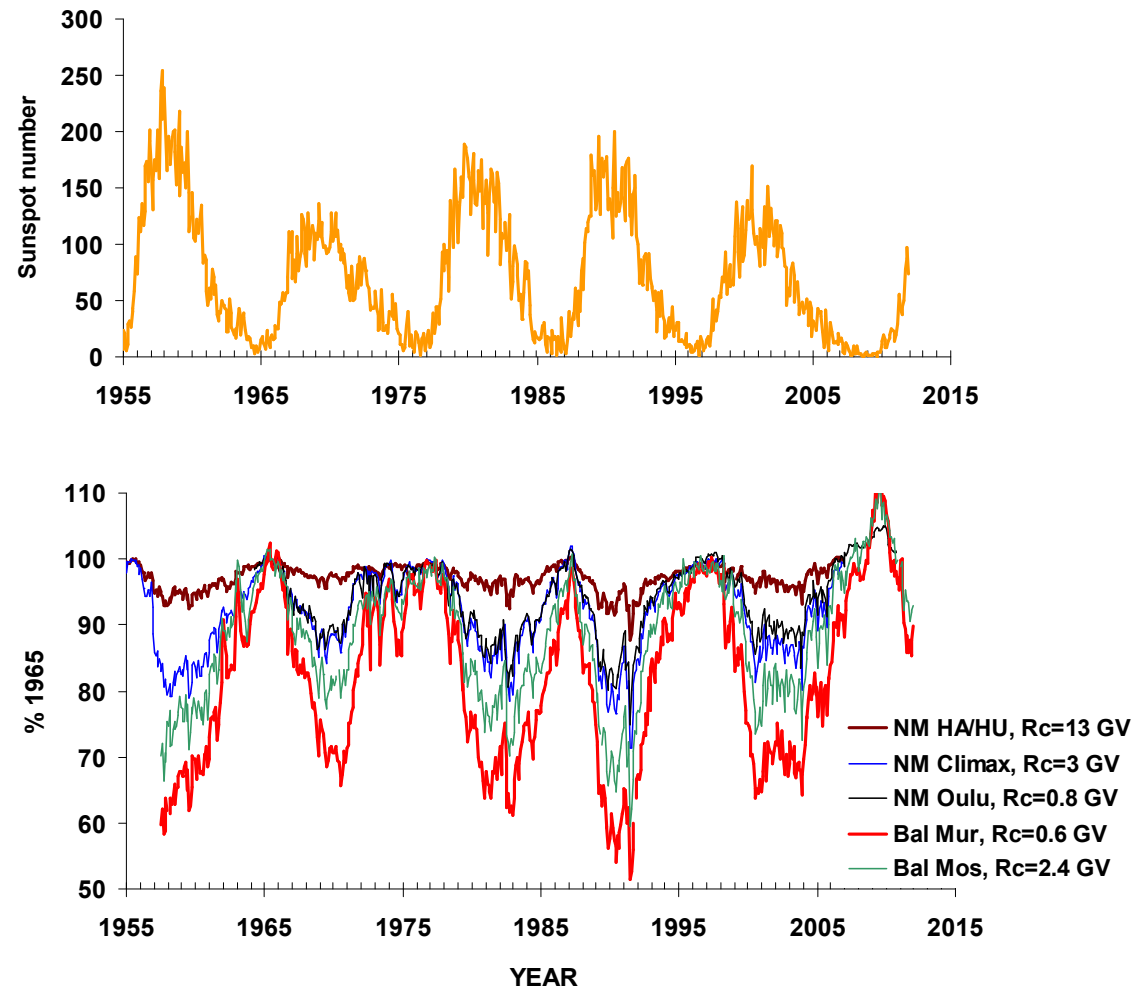

Fig. 6 Upper panel: International sunspot numbers (ftp://ftp.ngdc.noaa.gov/STP/SOLAR_DATA/SUNSPOT_NUMBERS/INTERNATIONAL/). Lower panel: $\mathrm{CR}$ fluxes as recorded by neutron monitors Huancayo/Haleakala $\left(\mathrm{R}_{c}=13\right.$ GV), Climax $\left(\mathrm{R}_{c}=3 \quad \mathrm{GV}\right)$ (http://ulysses.sr.unh.edu/NeutronMonitor/) and Oulu ( $\mathrm{R}_{c}=0.8 \mathrm{GV}$ ) (http://cosmicrays. oulu.fi), and by balloon-borne Geiger counters in the maximum of the transition curve in the atmosphere at polar (Murmansk, $\mathrm{R}_{c}=0.6 \mathrm{GV}$ ) and mid-latitude (Moscow, $\mathrm{R}_{c}=2.4 \mathrm{GV}$ ) locations. CR data are normalized to $100 \%$ in 1965.

Table 1 Magnitudes (in \%) of the 11-year modulation cycle as recorded by different cosmic ray detectors.

\begin{tabular}{l|cc|cccc}
\hline Instrument & $R_{c}(\mathrm{GV})$ & $\begin{array}{c}\text { solar cycles } \\
\text { covered }\end{array}$ & ground & $4-5 \mathrm{~km}$ & $10-12 \mathrm{~km}$ & $30-35 \mathrm{~km}$ \\
\hline Geiger (polar) & $0-0.6$ & $19-23$ & - & 9 & 16 & 45 \\
Geiger (Moscow) & 2.4 & $19-23$ & - & 10 & 15 & 30 \\
NM Oulu & 0.8 & $20-23$ & 15 & - & - & - \\
NM Moscow & 2.4 & $19-23$ & 14 & - & - & - \\
NM Huank/Haleak & 13 & $19-22$ & 4 & - & - & - \\
\hline
\end{tabular}




\section{Ion production rate at different altitudes, $R_{c}=0 \mathrm{GV}$}

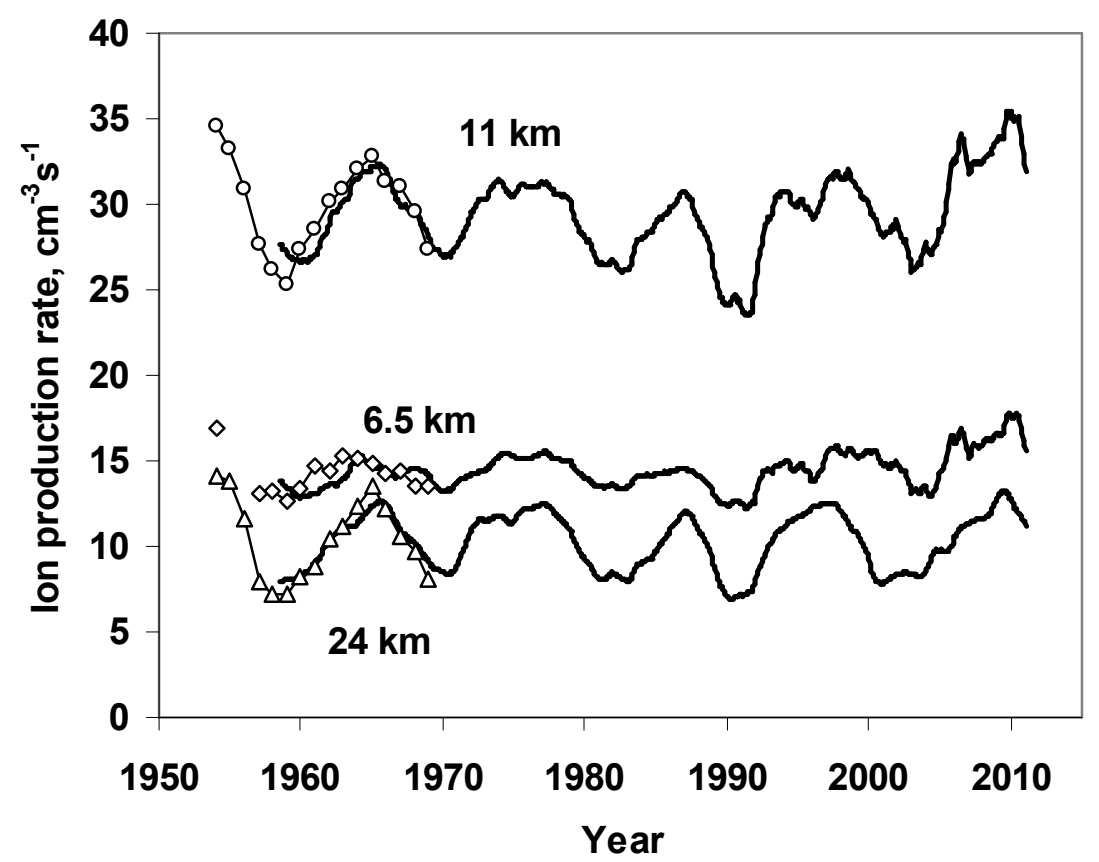

Fig. 7 Ionization rate in the atmosphere at altitudes of about 6.5, 11 and $24 \mathrm{~km}$ according to direct measurements by Neher (1971) (Thule, $R_{\mathrm{c}}=0$, open symbols), and as derived from the particle flux values measurements performed by the LPI group (Murmansk region $R_{\mathrm{c}}=0.6$ GV solid lines, 13 month smoothed).

with the unusually quiet solar conditions during that period (Gibson et al. 2011). The start of the modulation cycle after 2010 is clear in indicating the continuation of the normal solar dynamo operation.

The empirical relationship between the ionization rate $q$ and particle flux $J$ (see lower panel of Fig. 4) can be used to estimate a longer series of ionization rate at different altitudes, as shown in Fig. 7. The Lebedev Physical Institute measurements of the ionizing particle flux is consistent with fragmentary direct ionization measurements by Neher (1971). While the ionization rate is lower at the $24 \mathrm{~km}$ altitude than at $6.5 \mathrm{~km}$, the amplitude of the 11-year cycle is greater in the stratosphere. The flux of ionizing particles measured in the stratosphere is highly correlative with the ground-based NM data reflecting the modulation of GCRs (Usoskin et al. 2011). However, the correlation deteriorates in the troposphere, at altitudes below $15 \mathrm{~km}$ (Bazilevskaya et al. 2008), where enhanced variability at the inter-annual time scale is observed. The nature of this variability is not understood properly as yet and needs further work. 
Forbush decreases and 27-day recurrent variations

Sporadic releases of energy in the solar photosphere and/or corona, observed as solar flares or coronal mass ejections (CMEs) are usually accompanied by strong disturbances (interplanetary shocks), often driven by the magnetized CME ejecta, propagating through the interplanetary medium. Such disturbances effectively modulate GCRs in the heliosphere, virtually "sweeping" cosmic rays out, especially if several disturbances merge together forming a merged interaction region (MIR) or global MIR (GMIR). When such a disturbance passes near Earth, it leads to a suppression of GCR intensity known as a Forbush decrease (Cane 2000). The main feature of the Forbush decrease is a sharp decrease (duration of a few hours) in CR intensity, caused by the passage of the disturbance, followed by a gradual, nearly exponential, recovery, which may take several days or even weeks. Forbush decreases affect CR up to high energy of several tens of $\mathrm{GeV}$ as they are observed also at shallow-underground muon detectors (Usoskin et al. 2008). On top of that several smaller signatures can be found, such as pre-increase of the CR intensity (caused by the "collection" of CR particles in the shock upstream region) just before the event, the main phase may have a two-step structure, caused by the shock itself and by an ejecta passing the Earth. The exact shape of the Forbush decrease depends on the relative geometry of the interplanetary shock and Earth (Cane 2000). An example of a series of Forbush decreases is shown in Fig. 8. The CME that causes a Forbush decrease often originates in the same solar active region where a flare and/or a CME generating (SEP) occurs. Therefore, a strong CR intensity enhancement (ground level enhancement, GLE, see later) may be observed on the background and/or be followed by a Forbush decrease, as one can see in Fig. 8 for GLEs of 15th and 18th April 2001. Because of the lower energy of SEPs, GLEs are usually observed only by NMs at high and medium latitudes. The same interplanetary disturbance, which causes a Forbush decrease, may greatly distort the Earth's magnetosphere, therefore, Forbush decreases are highly correlated with geomagnetic disturbances, especially with storm sudden commencements.

Some statistics on Forbush decreases for the 23-rd solar cycle (Jordan et al. 2011) are shown in Fig. 9. The occurrence rate of Forbush decreases positively correlates with solar activity reaching its maximum around the maximum of the 11-year solar cycle. On the other hand, a noticeable increase in the occurrence rate is observed also at the declining phase of the solar cycle, due to recurrent Forbush decreases related to corotating fast streams of solar wind from long-lived coronal holes. A typical Forbush decrease has a magnitude around 4-5\% (up to 20\%) as observed by a ground-based NM and around $10 \%$ in the stratosphere as measured by a balloon-borne Geiger counter (Bazilevskaya et al. 2008).

A peculiar feature of the CR flux variation on shorter time scale is its 27-day quasiperiodic variability, observed as an intermittent 27-day quasi-periodic modulation of the cosmic ray intensity (Simpson 1998; Gil et al. 2005; Alania et al. 2011). The 27-day variation is related to the long-lived solar active formations on the Sun, such as coronal holes or large active regions. Due to the rotation of the Sun with a synodic period of about 27 days, such formations repeat their attitude regarding Earth with this period. Coronal holes form a source of fast solar wind streams, which can catch up solar wind plasma from slow streams emitted earlier, leading to their interaction and formation of a corotating region with a shock front. Such corotating regions slightly modulate the CR flux near Earth with the period of 27 days (see Fig. 10). When the coronal hole disappears, the recurrent 27-variation can also cease to appear later at the new 


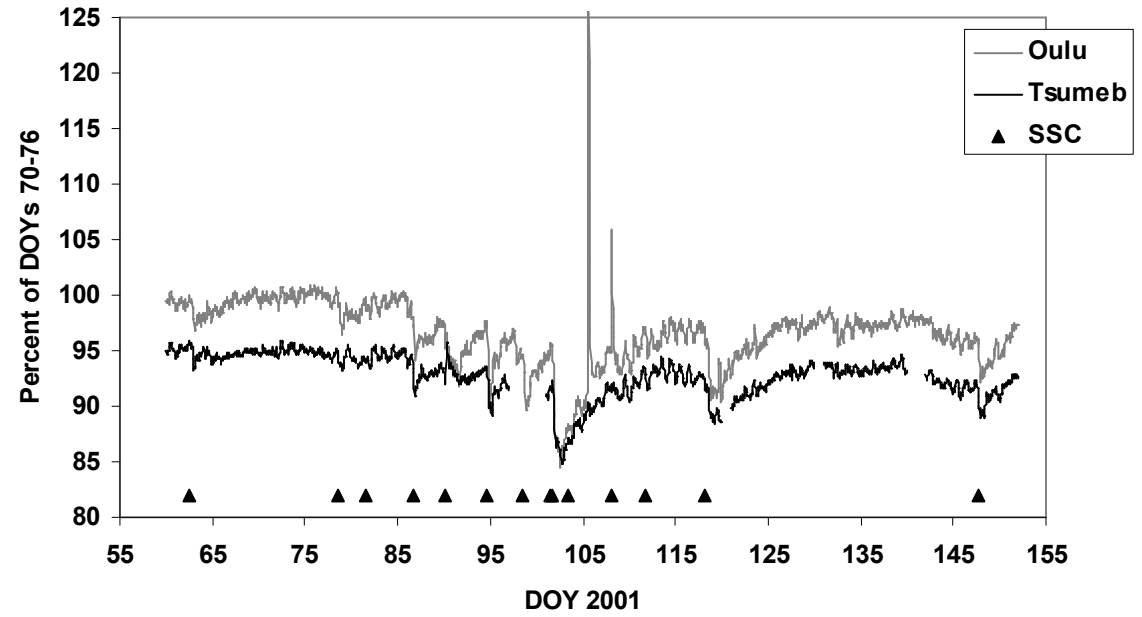

Fig. 8 Series of Forbush decreases in March - April 2001 as observed by the Oulu $\left(R_{c}=0.8 \mathrm{GV}\right)$ and Tsumeb $\left(R_{c}=9.1 \mathrm{GV}\right)$ neutron monitors (http://cosmicrays.oulu.fi/, http://www.nwu.ac.za/neutron-monitor-data). Count rates are normalized to the average value from DOYs (day of year) 70 to 76 (Oulu - 100\%, Tsumeb - 95\%). Black triangles in the bottom denote storm sudden commencements (ftp://ftp.ngdc.noaa.gov/STP/SOLAR_DATA/SUDDEN_COMMENCEMENTS).
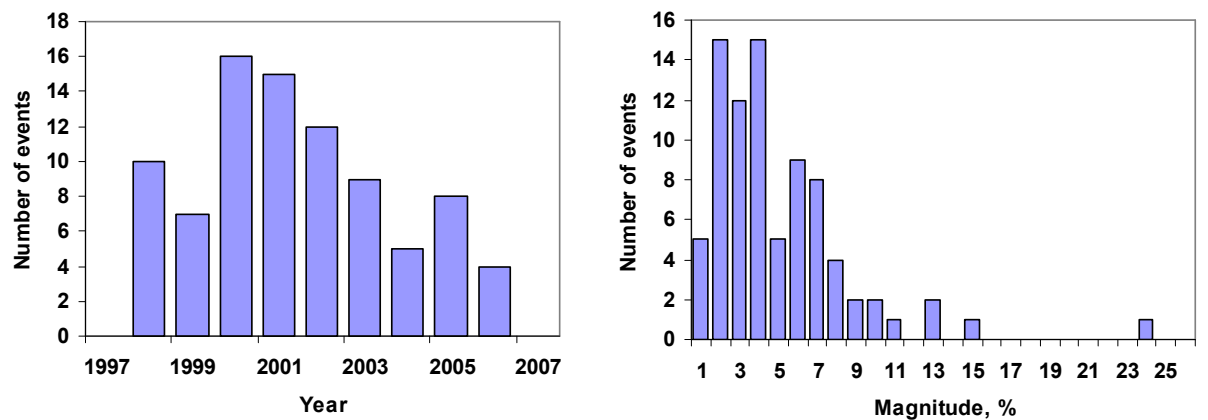

Fig. 9 Statistics of Forbush decreases during solar cycle 23 (based on Jordan et al. 2011). Left panel: The number of observed Forbush effects for years 1998 through 2006. Right panel: Histogram of the Forbush decrease magnitudes which are the maximum variations of CR intensity during the event (Belov 2009), as observed at the polar station McMurdo NM.

phase. This feature is usually more distinctive and persistent during the descending or minimum phases of the solar activity cycle. Forbush decreases also often re-occur with roughly 27-day period as the parent active region persists on the rotating Sun. 


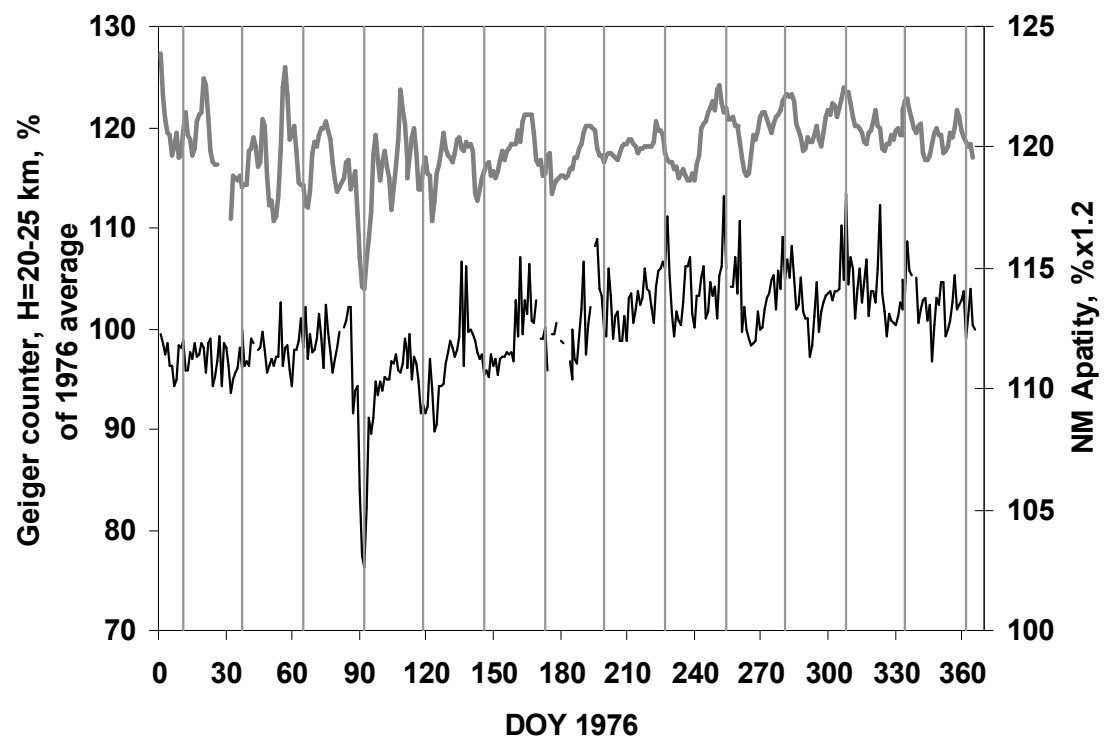

Fig. 10 An example of 27-day variations of the CR intensity as observed by the balloon-borne Geiger counter in the stratosphere (20-25 km altitude, thin black curve) and the Apatity NM (thick grey curve, http://pgia.ru/CosmicRay). Values are normalized to the average value for 1976 (Geiger counter - 100\%, Apatity NM - 120\%). Vertical lines are plotted every 27 days.

\subsubsection{Solar energetic particles}

Strong increases of the intensity of energetic particles can sporadically occur near Earth, due to the arrival of solar energetic particles (SEPs also known as Solar Cosmic Rays (SCRs), or Solar Proton Events (SPEs)). SEPs are associated with fast powerful energy release phenomena on the Sun, solar flares and/or CMEs. SEPs are particles accelerated by magnetic reconnection or by stochastic mechanisms in the parent flare region, or by a CME-driven shock in the solar corona and interplanetary space (Miroshnichenko and Perez-Peraza 2008). Although the elemental composition of SEPs changes from one event to another, it is dominated $(>90 \%)$ by protons. The energy of SEPs extends from $\approx 1 \mathrm{MeV}$ to several $\mathrm{GeV} /$ nucleon. The energy spectrum of SEPs covers more than four orders of magnitude in energy and more than 8 orders of magnitude in intensity. Accordingly, it is hardly possible to measure the entire SEP energy range with a single instrument. SEPs with energies below several hundred $\mathrm{MeV}$ are well measured onboard numerous spacecraft, while balloon-borne detectors are more suitable to detect SEPs with energies between 100 and $500 \mathrm{MeV}$. The most energetic SEPs (above 1000 $\mathrm{MeV}$ ) can initiate the nucleonic-electromagnetic cascade in the atmosphere, leading to the nucleon and muon components potentially recordable by ground-based detectors (neutron monitors) as a ground level enhancement (GLE). The time-profile, spectrum and elemental composition of a SEP event depends on many factors, most important being the acceleration mechanism and the relative Sun-Earth geometry (Vainio et al. 2009). 


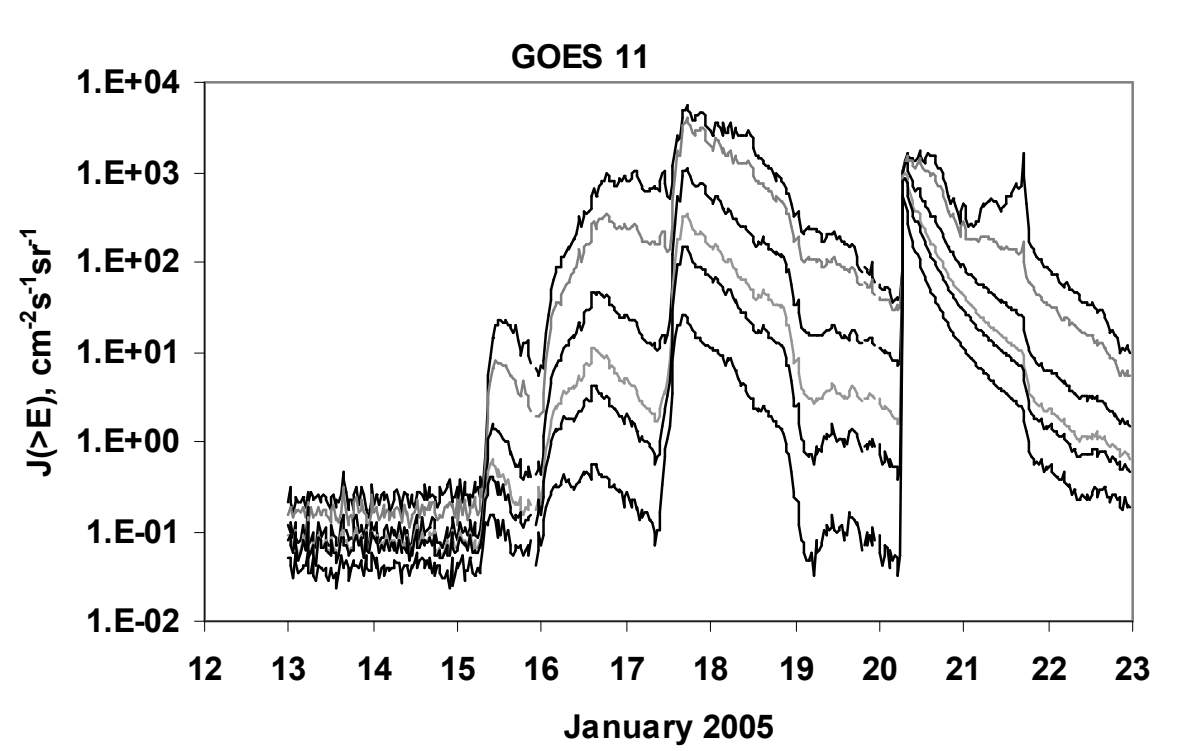

Fig. 11 Time profiles of proton intensities during the series of SEP events in January 2005 as measured onboard the GOES 11 spacecraft. Energy of protons from top to bottom: above 5, 10, 30, 50,60, and $100 \mathrm{MeV}$, respectively (http://spidr.ngdc.noaa.gov/spidr).

SEP events are fairly often observed in series being associated with the same parent active region on the Sun. An example of this is given by the burst of activity in January 2005 (Figs. 11 and 12), where several SEP events were observed to originate from the same active region (NOA AR 10720) on the Sun. One can see that the largest (within this group of events) flux of low-energy SEPs was achieved for the gradual event of 17th January, while the higher energy particles were more abundant for the impulsive event of 20th January. It should be noted that very energetic protons were produced during the event of 20th January when a strong GLE (second largest ever observed, about $3200 \%$ enhancement at the South Pole NM - Plainaki et al. (2007)) was recorded by the NM network. One can see from Fig. 12 that the energy spectrum of SEPs was very hard. On the other hand, the spectrum is flat in the low-energy range 10-100 MeV, making the low-energy fluence for this event quite modest (Mewaldt et al. 2007). We note that such a situation is typical for GLE events (Bazilevskaya and Sladkova 2003). Solar energetic particles with energies below $100 \mathrm{MeV}$ lose their energy in the atmosphere at altitudes above $30 \mathrm{~km}$, mostly due to ionization of the ambient air (Winckler 1956; Charakhchyan 1964; Bazilevskaya et al. 2008; Mishev and Usoskin 2013). For example, protons with energies 10, 30, and $100 \mathrm{MeV}$ cannot penetrate the atmosphere deeper than about 58, 45, and $32 \mathrm{~km}(0.15,1.0$, and 8.6 $\mathrm{g} / \mathrm{cm}^{2}$ of atmospheric depth), respectively. Moreover, such particles can penetrate only in the polar cap region where there is no geomagnetic shielding. Since such low-energy particles are much more abundant in the SEP spectrum (Fig. 12), the strongest effect of SEP events is ionization produced in the upper polar stratosphere and mesosphere at about 40-90 km altitude (e.g., Quack et al. 2001; Velinov et al. 2013). The impact of SEPs on the atmosphere is considered in Section 3.2.3 of this paper. 


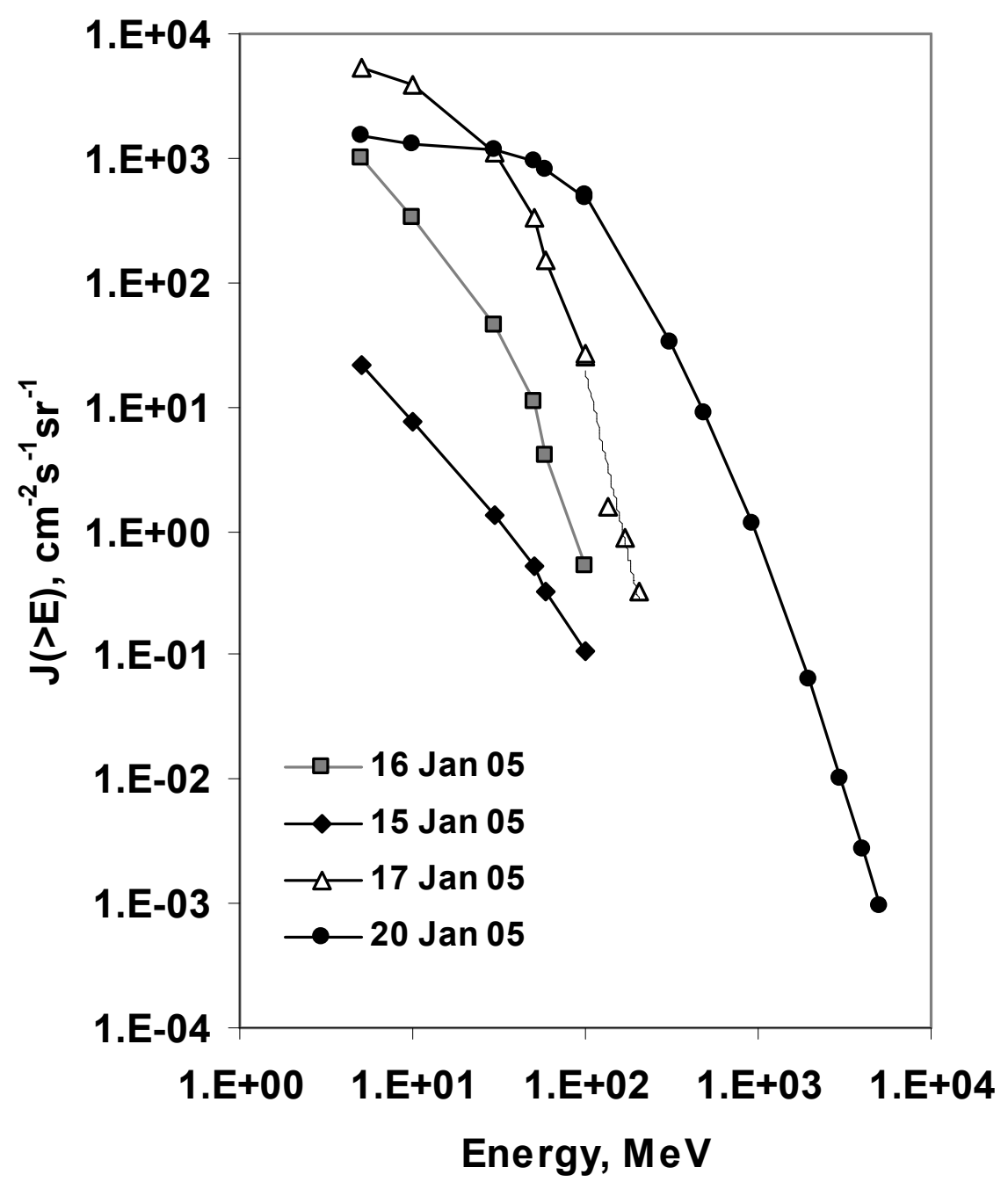

Fig. 12 Integral energy spectra of SEP events shown in Fig. 11, corresponding to the maxima of the intensity-time profiles. Points below $100 \mathrm{MeV}$ are based on the GOES 11 data. For the events of 17 th and 20 th January, protons $(>100 \mathrm{MeV})$ were recorded by the stratospheric detector of the LPI group. The event of 20th January was also recorded by the NM network as GLE 69.

Solar energetic particles with energies above $100 \mathrm{MeV}$ intrude into the polar atmosphere where balloon-borne detectors observe them at stratospheric altitudes through great changes of the transition curve, as shown in the left panel of Fig. 13 for the event of 20th January 2005. The particle intensity increases in the polar stratosphere sometimes by an order of magnitude in comparison with the quiet time. Subtracting the background caused by GCRs, one can estimate energy spectra of the incoming solar 

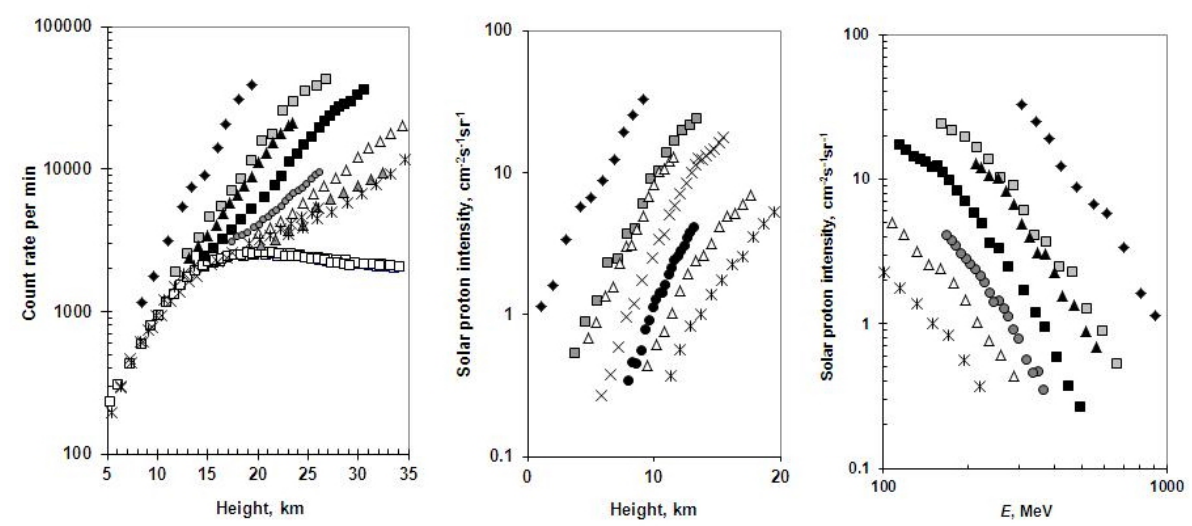

Fig. 13 Solar energetic particles in the polar atmosphere for the SEP event of 20th January 2005. Left panel: Balloon-borne measurements by LPI in the northern polar region during the SEP or GLE event of 20th January 2005. White squares are the background from GCRs, other symbols correspond to different times of observations. Middle panel: SEP intensity vs. height. Right panel: SEP energy spectra as derived from the data shown on the left panel.

protons (right panel in Fig. 13). Fig. 14 shows the intensity time profile of this SEP event. One can see that more energetic particles can penetrate deeper into the atmosphere, but the duration of an enhancement becomes shorter, as the energy spectrum softens with time. The GLE event at the ground-level lasted for a few hours at most whereas a typical SEP event in the stratosphere usually lasts several days. Strong SEP events often occur in series, so that the charged particle intensity in the stratosphere can be enhanced for several weeks, as e.g. happened in the autumn of 1989. The intensity of less energetic $<100 \mathrm{MeV}$ protons remains enhanced even longer. For example, the enhanced fluxes of $>10 \mathrm{MeV}$ protons were observed in total for more than 110 days in 2001 .

The majority of SEP events are not observed in the atmosphere as they have a soft energy spectrum, since the number of SEP events decreases with increasing the SEP energy. Generally, the peak intensity distribution for $>10 \mathrm{MeV}$ solar protons can be approximated by a power law with the index $g=-1.34$ (Bazilevskaya 2005). The occurrence rate of SEP events can be observed in Fig. 15. From solar cycle to solar cycle, the number of SEP events recorded in the atmosphere varied from 21 to 32, the number of GLEs from 12 to 16, whereas the number of events with intensity $J(>10 \mathrm{MeV}) \geq 1\left(\mathrm{~cm}^{2} \mathrm{~s} \mathrm{sr}\right)^{-1}$ varied between 120 and 150 . Data for $>10 \mathrm{MeV}$ solar protons are taken from Logachev $(1982,1990,1998)$, for the cycle 23 these data are preliminary. Data on solar protons with energies above $1 \mathrm{GeV}$ are deduced from GLE events recorded by NMs using the specific yield functions of a NM (Lockwood et al. 1974). These values should be considered as estimations as they were obtained under assumption of the SEP flux isotropy outside the Earth's magnetosphere, which is not always correct. The currently progressing 24 th solar cycle depicts a slow rise, at least to the level of moderate cycles in the 19-th century, without an indication of new Grand minimum. Until the end of 2014 only one GLE (17 May of 2012) was recorded for certain in the cycle 24 (the second possible event, not finally confirmed, occurred on 6 


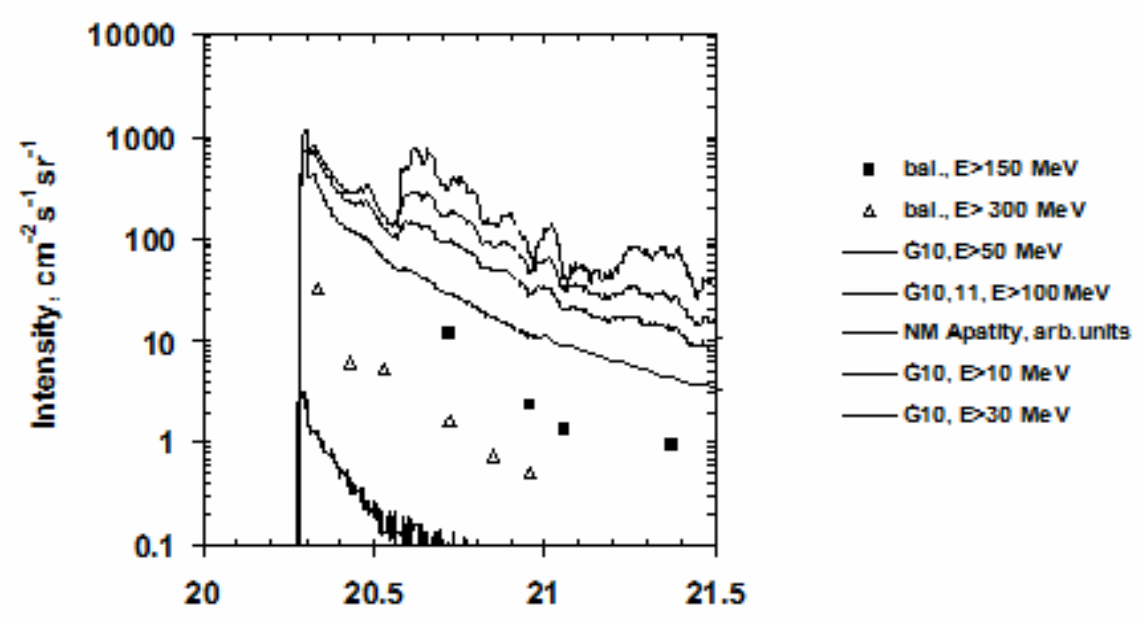

January 2005

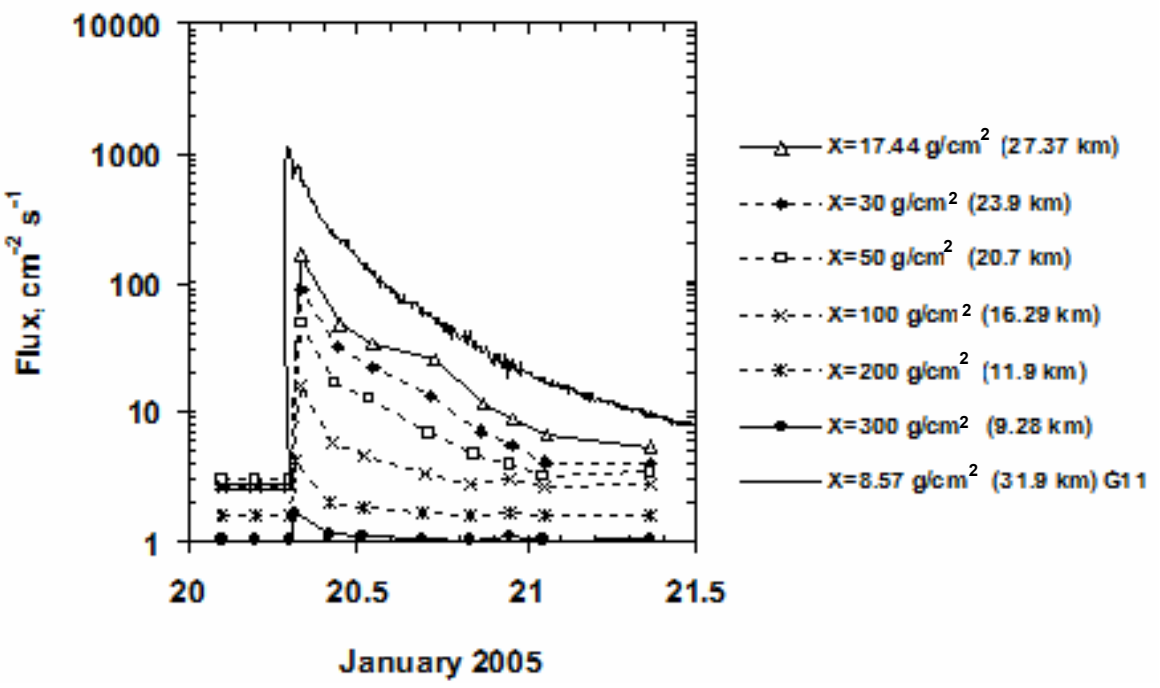

Fig. 14 Time profiles of the SEP intensities for the GLE event of 20th January 2005. Upper panel: Intensities of solar protons of different energies, as observed by the the GOES satellite (http://spidr.ngdc.noaa.gov/spidr), by balloon-borne detectors by LPI, and by the Apatity NM (http://pgia.ru/CosmicRay). Lower panel: Enhanced particle fluxes as observed at different altitudes in the atmosphere. Reprinted from Bazilevskaya et al. (2010), with permission from Elsevier. 
January of 2014). However, the number of SEP events with $J(10 \mathrm{MeV}) \geq 1\left(\mathrm{~cm}^{2} \mathrm{~s} \mathrm{sr}\right)^{-1}$ is more than 70 to the end of 2014 .

\subsubsection{Modelling energetic particles and the cosmic ray induced ionization}

When an energetic particle penetrates the atmosphere, it first traverses through the upper rarefied layer of the atmosphere, ionizing the ambient air. During this phase the energetic particles move in a straight line, losing energy for ionization. Low energy particles can be completely stopped in the upper atmosphere due to these ionization losses. This process can be modeled in a simple way using an analytical approach (see, e.g., Velinov and Mateev 1990; Vitt and Jackman 1996). However, if the primary particle possesses sufficient energy (about a hundred $\mathrm{MeV}$ ) to traverse about $100 \mathrm{~g} / \mathrm{cm}^{2}$ of matter, it accidentally collides, if its energy is still sufficient, with a nucleus of one of the atmospheric gases, where the most abundant nuclei are nitrogen and oxygen. In such a nuclear collision, different secondary products can be created, having different fates in the atmosphere (Dorman 2004). If the energy of the secondaries is still high enough, they may undergo further collisions and interactions forming the so-called atmospheric cascade. Because of the thickness of Earth's atmosphere $\left(1033 \mathrm{~g} / \mathrm{cm}^{2}\right)$ the number of subsequent interactions can be large leading to a fully developed cascade consisting of a variety of secondary particles. This process is well understood but cannot be modelled analytically and requires a full Monte-Carlo approach (Usoskin et al. 2004; Desorgher et al. 2005; Usoskin and Kovaltsov 2006; Usoskin et al. 2010). It is common to separate three main components: the "soft" or electromagnetic component consisting of electrons, positrons and photons; the "hard" or muon component consisting of muons (pions are short-lived and decay almost immediately); and the "hadronic" nucleonic component consisting mostly of super-thermal protons and neutrons. All the charged secondaries ionize air, leading to a complicated process of ionization in the low and middle atmosphere (see detail in Bazilevskaya et al. 2008).

Therefore, the process of cosmic ray induced ionization can be modelled by different approaches depending on the particle's energy, as described below. In all models, the ionization energy losses of particles (either primary or secondary of different nature) are converted into the production of ion pairs, assuming that one ion-electron pair is produced, on average, per each $35 \mathrm{eV}$ of deposited energy (Porter et al. 1976). Such models yield ionization rates, however in order to obtain the ion concentration, recombination processes need to be considered, but this is usually treated separately depending on the exact ambient conditions.

The cosmic ray induced ionization (CRII) at a given location is usually represented in numerical models via the ionization function $Y$ (Usoskin and Kovaltsov 2006)

$$
Q(h)=\sum_{\mathrm{A}} \int_{T_{\mathrm{c}, \mathrm{A}}}^{\infty} J_{\mathrm{A}}(T) \cdot Y_{\mathrm{A}}(T, h) d T,
$$

where the summation is performed over different $A$-th species of CR (protons, $\alpha$ particles, etc.), $Y_{\mathrm{A}}(h, T)$ is the ionization yield function (the number of ion pairs produced at altitude $h$ in the atmosphere by CR particles of the type $A$ with kinetic energy $T$ and unit flux of $\mathrm{C}$ ). Integration is performed above $T_{\mathrm{c}, \mathrm{A}}$, which is the kinetic energy of a particle of type $A$, corresponding to the local geomagnetic rigidity cutoff $P_{\mathrm{c}}$ (Smart et al. 2006). 

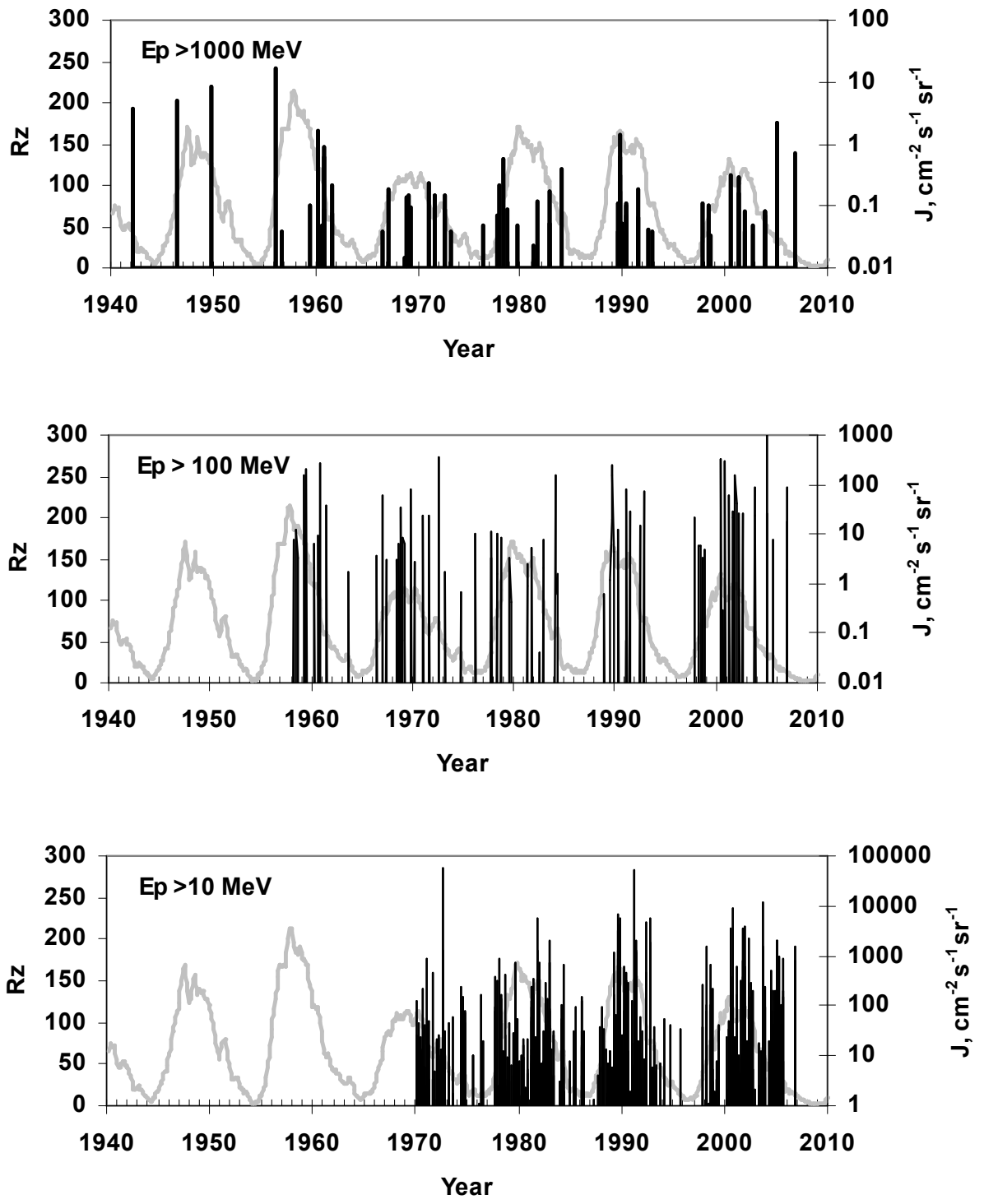

Fig. 15 Upper panel: Occurrence of SEP events as observed by ground-based neutron monitors (GLE, solar proton energy $E p>1000 \mathrm{MeV}$, number of events from solar cycle to solar cycle varies from 12 to 16, data of SEP with energies above $1 \mathrm{GeV}$ are deducted). Middle panel: Occurrence of SEP events in the stratosphere by the LPI instrument $(E p>100 \mathrm{MeV}$, number of events from solar cycle to solar cycle varies from 21 to 32). Lower panel: Occurrence of SEP events in space by satellites (lower panel, events with $J(E p>10 \mathrm{MeV}) \geq 1\left(\mathrm{~cm}^{2} \mathrm{~s} \mathrm{sr}\right)^{-1}$ - Logachev (1982, 1990, 1998), number of events from solar cycle to solar cycle varies from 120 to 150). Each vertical bar depicts an SEP event, and its length corresponds to the SEP intensity (right-hand axis) in the event's maximum. Grey curves are the sunspot number $\mathrm{R}_{z}$ (left-hand axis). 
Analytical model

A typical analytical calculation (e.g., Vitt and Jackman 1996) of the ionization rate in the upper atmospheric layer ( $\mathrm{a}$ few $\mathrm{g} / \mathrm{cm}^{2}$ of matter or a few $\mathrm{hPa}$ ), using a thin target approximation is briefly discussed here. Elastic scattering processes are neglected in the apporach, assuming that an energetic particle moves straight losing energy for ionization of the ambient air. The particle can be lost either by losing all its kinetic energy (thermalization) or by a nuclear collision (no secondaries of such a collision are considered though).

Let us consider a primary cosmic rays particle of type $A$ with the kinetic energy per nucleon $T$ penetrating to the atmosphere at the zenith angle $\theta$. Its probability to survive, against inelastic process, until its kinetic energy becomes $T^{\prime}$ is given as

$$
p\left(T, T^{\prime}, A\right)=\exp \left(-\int_{T^{\prime}}^{T} \frac{d T}{\frac{d T}{d x}(T, A) \cdot \lambda_{\text {in }}(T, A)}\right),
$$

where $\frac{d T}{d x}(T, A)$ is the stopping power due to ionization losses, and $\lambda_{\text {in }}(T, A)$ is the path-length for inelastic nuclear collisions, respectively, as tabulated, e.g., by Janni (1982). Note that many models dealing with lower energy particles neglect the nuclear collisions, implicitly assuming $p=1$. This is a reasonable assumption for energies below about $10 \mathrm{MeV}$ but leads to serious errors if more energetic particles are involved. The thickness $\mathrm{x}$ (in $\mathrm{g} / \mathrm{cm}^{2}$ ) traversed by the particle with initial energy $\mathrm{T}$ and final energy $T^{\prime}$ along its trajectory can be expressed as:

$$
x=R(T)-R\left(T^{\prime}\right) \equiv \frac{h}{\cos \theta},
$$

where $R(T)$ is the path-length of a particle with energy $T$ due to ionization losses (Janni 1982), and $h$ is the atmospheric depth in $\mathrm{g} / \mathrm{cm}^{2}$ (related to the barometric pressure). Then one can derive that the ionization rate $q$ is defined as

$$
\frac{d q}{d x}(x, T, A)=\frac{1}{35 \mathrm{eV}} \cdot \frac{d T}{d x}\left(T^{\prime}, A\right) \cdot p\left(T, T^{\prime}, A\right),
$$

where $T^{\prime}$ is defined from the equation (4).

Finally, the ionization yield function for the isotropic flux of particles impinging on the top of the atmosphere is defined as

$$
Y_{\mathrm{A}}(T, h)=2 \pi \int_{0}^{1} \frac{d q}{d x}(x, T, A) d \cos \theta
$$

Applying this result to the observed spectrum of energetic particles, one can calculate the corresponding ionization rate (Eq. 2).

\section{Monte-Carlo models}

The atmospheric cascade can be properly modelled only by a full Monte-Carlo model. Earlier analytical approximations (O'Brien 1979) attempted to model the cascade via the Boltzman equation, but such models become less reliable in the lower atmosphere. 


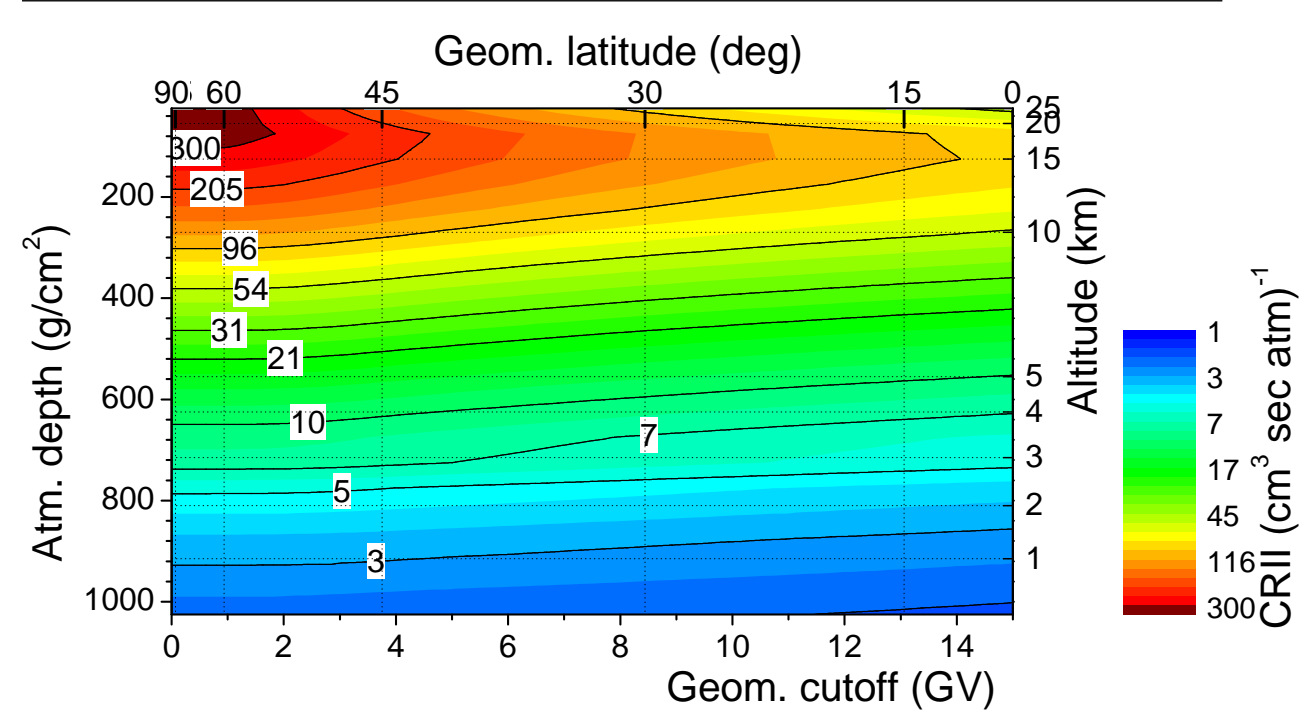

Fig. 16 Modelled CRII as a function of the atmospheric depth $h$ (left axis) or altitude (right axis) and the geomagnetic rigidity cutoff $R_{\mathrm{C}}$ (lower axis) or geomagnetic latitude (upper axis) for medium CR modulation. Modified from Bazilevskaya et al. (2008).

Note that the value of CRII (Eq. 2) is defined by three main variables: altitude (quantified via the atmospheric depth $h$ ), geographical location (via the local geomagnetic rigidity cut-off $R_{\mathrm{C}}$ in the integration limits), and time (via the variability of the integrand energy spectrum $J$ ). Since these three variables are mutually independent, they can be separated, which helps to solve the problem numerically in an efficient way by producing pre-calculated 3D look-up tables (http://cosmicrays.oulu.fi/CRII/CRII.html).

The spatial distribution of the CRII is shown in Fig. 16. The maximum ionization appears at about $18-20 \mathrm{~km}$ altitude in the polar region, where the ionization rate is several orders of magnitude greater than that at sea level. The difference in the ionization rate between the equatorial and polar regions is of the order of a factor 3-5. Variability of CRII over a solar cycle is from 10-20\% in the troposphere up to a factor of 2 in the polar stratosphere.

Presently there are two basic numerical approaches to the CRII Monte-Carlo simulation. Details of the method are given elsewhere (Usoskin and Kovaltsov 2006; Bazilevskaya et al. 2008). One is the ATMOCOSMICS/ PLANETOCOSMICS model developed by the Bern group (Desorgher et al. 2005) on the basis of the GEANT-4 simulation package. Another one is the CRAC (Cosmic Ray Atmospheric Cascade) model developed by the Oulu group (Usoskin et al. 2004; Usoskin and Kovaltsov 2006; Usoskin et al. 2010) on the basis of CORSIKA+FLUKA Monte-Carlo packages. Several clones of these codes exist (e.g., Mishev and Velinov 2011). Comparison between the ATMOCOSMICS and CRAC models vs. fragmentary direct balloon-borne measurements of the ionization rate in a high latitude region (geomagnetic cutoff rigidity $\left.P_{\mathrm{c}}<1.5 \mathrm{GV}\right)$ is shown in Fig. 17.

Modelled and measured CRII rates are in good agreement (Bazilevskaya et al. 2008). Simulations agree with each other within $10 \%$, which can be attributed to the different atmospheric models used and, to a less extent, to different cross-section approximations in CORSIKA and GEANT-4 packages (Usoskin et al. 2009). 


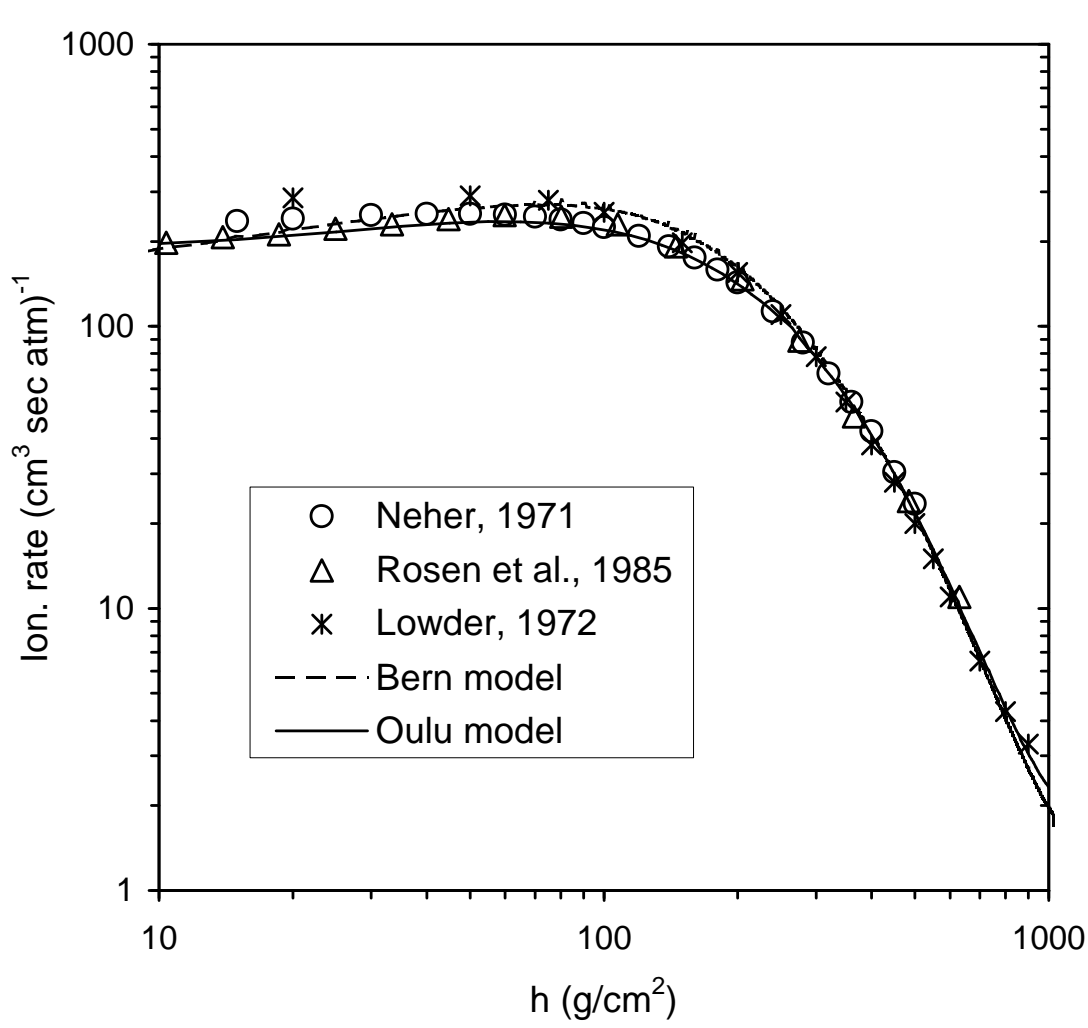

Fig. 17 Cosmic ray induced ionization rates in a high-latitude region $\left(P_{\mathrm{c}}<1.5 \mathrm{GV}\right)$ for a typical solar maximum $(\phi \approx 1000 \mathrm{MV})$. Symbols represent direct measurements as denoted in the legend, while curves correspond to the calculations using the ATMOCOSMICS (dashed) and CRAC (solid) Momte-Carlo models. Adapted from Usoskin et al. (2009).

There is another GEANT-4 based model - AIMOS (Atmospheric Ionization Model of Osnabrück, (Wissing and Kallenrode 2009)), which is commonly used to compute the effect of auroral, magnetospheric and solar particles in the middle and upper polar atmosphere. The AIMOS model does not consider particles with energies $>500 \mathrm{MeV}$ and thus cannot be applied to study the effect of GCRs (which is minor in the upper polar atmosphere but dominant in the tropo- and stratosphere). Its use for GLE events should be taken with caution because of the limited energy range. The advantage of AIMOS is it is ability to create a temporally and spatially variable 3-dimensional global ionisation source input to atmospheric models, based on POES satellite measurements for lower energy particles. Care should be taken however, due to possible inaccuracies in POES data due to contamination of the detector channels during energetic particle events (Rodger et al. 2010).

Therefore, 3D models (CRAC:CRII or PLANETOCOSMIC) allow to compute the ionization effects caused by galactic cosmic rays as well as by solar cosmic rays during SEP/GLE events at different atmospheric heights. The ionization effect of GLE events is important only in the polar atmosphere, where it can be dramatic in the middle and upper atmosphere (above $30 \mathrm{~km}$ ) during major GLE events (Usoskin et al. 2011). 


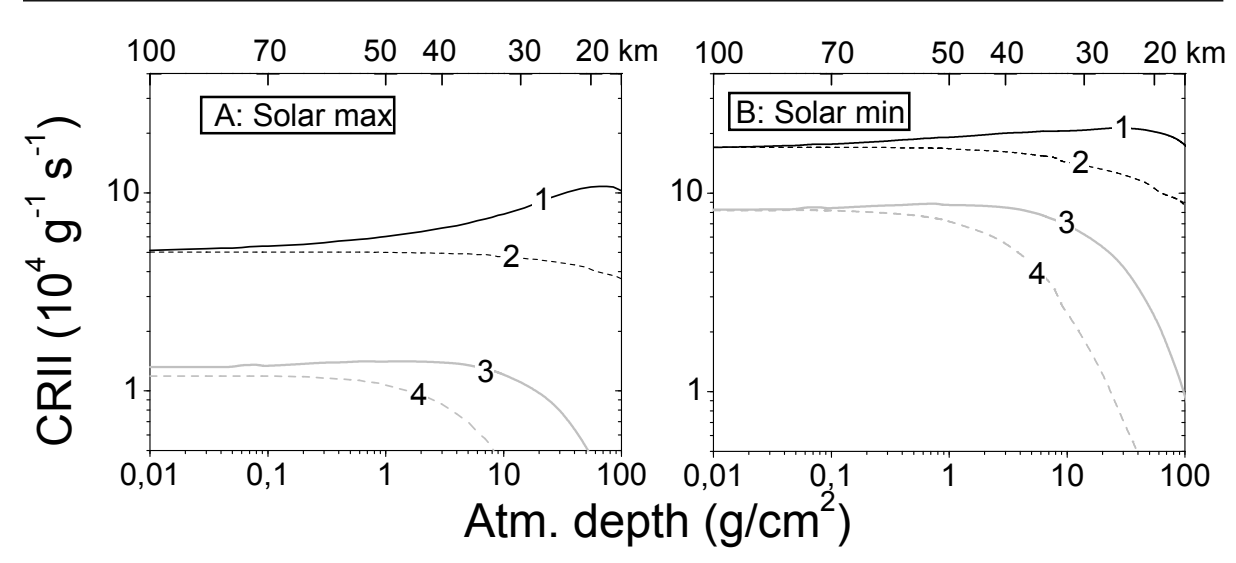

Fig. 18 Ionization rate in the polar upper atmosphere induced by galactic cosmic ray protons for solar maximum (panel A) and minimum (panel B) conditions, computed by different models. The upper scale depicts approximate height in $\mathrm{km}$ for the standard US atmosphere. The curves are denoted as: (1) Full Monte-Carlo model (e.g., Usoskin et al. 2010); (2) Full analytical thin-target model (e.g., Vitt and Jackman 1996); (3) Monte-Carlo model considering only protons with energies below $500 \mathrm{MeV}$ (e.g., Wissing and Kallenrode 2009); (4) Analytical thin-target model considering only primary cosmic rays with energies below $500 \mathrm{MeV}$. After Usoskin et al. (2010) and re-used with permission from Wiley.

\section{Comparison of different approaches}

We note that while the full Monte-Carlo models are universal in the sense that they can deal with all types and energies of primary precipitating particles, other simplified or truncated models have been developed to deal with specific types of the ionizing radiation. They have limited ranges of validity (typically altitudes above $35 \mathrm{~km}$ and particle energy below a few tens of $\mathrm{MeV}$ ) and may become invalid when used outside these ranges. However, it is quite typical that simplified parameterization models, originally intended to work with low-energy precipitating particles in the polar upper atmosphere, are incorrectly used to assess the ionization by energetic particles (SEP/GLE events or even GCR) down to the lower stratosphere (e.g., Calisto et al. 2012; Verronen et al. 2011b). Here we emphasize that this may lead to large errors.

Fig. 18 presents a comparison of the CRII rates in the upper $10 \%\left(100 \mathrm{~g} / \mathrm{cm}^{2}\right)$ of the atmosphere due to GCR protons as computed using different models. One (curve 1) is the full Monte-Carlo model (Usoskin et al. 2010) described in Section 2.1.5. One can see an increase of the ionization at a depth of around $90 \mathrm{~g} / \mathrm{cm}^{2}$ due to development of the atmospheric cascade. Another curve (2) represents the analytical model (e.g., Vitt and Jackman 1996) as described in Section 2.1.5. While the two models agree for the SEPs and in the very upper part of the atmosphere, the analytical model underestimates the ionization rate by energetic particles progressively downwards because of neglecting the cascade development. The difference can be as large as a factor of $2-3$. We note that some of the ionization models focused upon the middle and upper atmosphere consider a truncated energy range of the energetic particles, below $500 \mathrm{MeV}$. An example of such a truncated models is the AIMOS model (Wissing and Kallenrode 2009). Curve (3) in Fig. 18 corresponds to such a model. One can see that, despite the correct method applied (full Monte-Carlo), such a truncated model grossly 
underestimates the ionization effect of GCRs - both in magnitude (by a factor of 2-5) and in the penetration depth. An analytical model with the truncated energy range (curve 4) is similar to that in underestimating the ionization effect. On the other hand, truncated and simplified models can be well used for solar energetic or magnetospheric precipitating particles with very soft spectrum (Bazilevskaya et al. 2008).

Accordingly, by considering only low energy particles ( $<500 \mathrm{MeV}-$ grey curves), one can more or less correctly model the effect of solar energetic and magnetospheric particles, but grossly underestimate the ionization rate due to GCRs, even in the uppermost part of the atmosphere. Generally, a full Monte-carlo model is always recommended to be used for the thick Earth's atmosphere. Note, however that GCRs play only a minor role in the overall ionization in the upper atmosphere. 


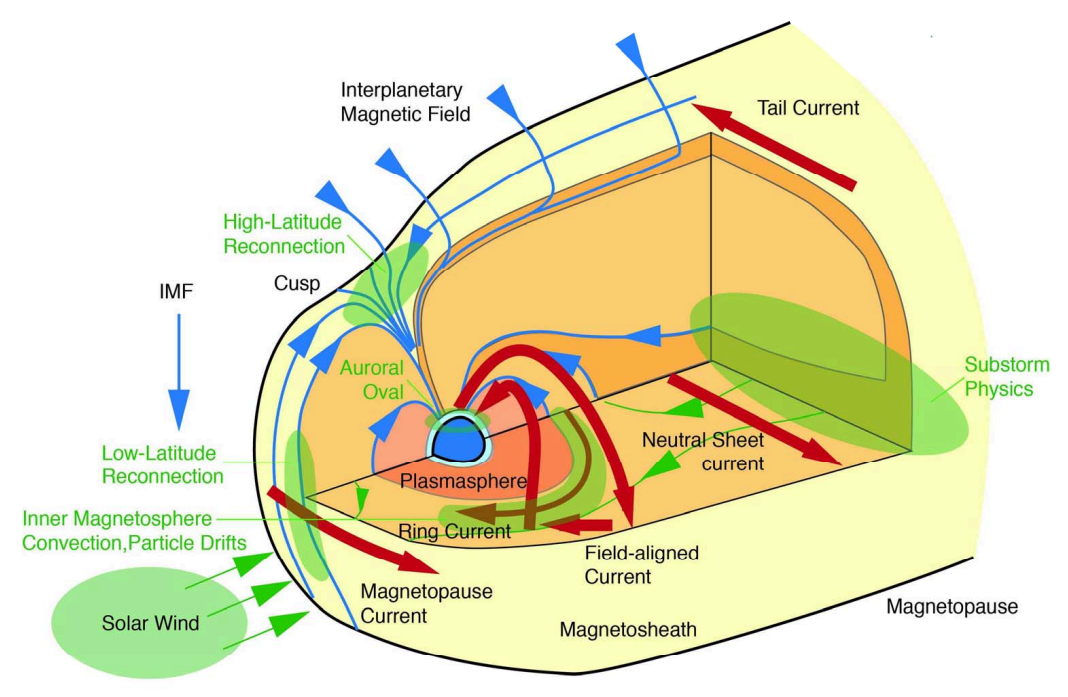

Fig. 19 Magnetosphere with its major plasma regions and physical processes (shaded in green), together with the main current systems (red arrows). Modified after Frey (2007).

\subsection{Sources of the electrons in the Earth's atmosphere}

Dynamic coupling of the solar wind with the Earth's magnetosphere (Fig. 19) results in acceleration of charged particles partly precipitating into the atmosphere. An important role in the atmospheric processes is played by precipitating populations of auroral electrons and radiation belt electrons. Auroral electrons are magnetotail particles from the plasma sheet, which are further accelerated in the magnetosphere, before precipitating into the atmosphere.

Forcing by the solar wind modifies the Earth's intrinsic geomagnetic field in nearEarth space, creating a cavity called the magnetosphere, (Fig. 19), which is filled with plasma of both solar wind and ionospheric origin. Precipitation of both electrons and ions into the atmosphere occurs from various regions of the magnetosphere, due to various mechanisms, some of which are still poorly understood, although recent satellite missions have increased knowledge on relations e.g between the diffuse precipitation features and wave-particle interactions. There are various regions in magnetosphere, (Fig. 19), where process of energy exchange exists. Magnetic reconnection may occur at low latitudes on dayside as well as at high latitudes and in distant magnetotail, which is the region of auroral substrom physics. In the magnetospheric current systems shown in Fig. 19 the field aligned currents relate to precipitation seen as the auroral oval, whereas precipitation on open field lines occurs from magnetosheath or directly from solar wind. In the inner magnetosphere, one may have precipitation of radiation belt particles for example in wave-particle interactions, or weaker precipitation from ring current scattering. Interestingly, energies of the precipitating particles often exceed 
those available from solar wind particles, so that various acceleration mechanisms must be present in the magnetosphere. For the needs of understanding the atmospheric effects, one needs a description of the fluxes and energy spectra of the precipitating particles, the geographical regions of occurrence of precipitation, and both short and long term variability of the precipitation.

The various plasma regimes of the magnetosphere, related to particle precipitation are summarized by Lyons (1997) as polar rain, direct precipitation of solar wind electrons on open polar cap field lines; polar energetic particles at energies higher than $100 \mathrm{keV}$ during solar particle events; cusp precipitation from magnetosheath plasma along recently dayside opened field lines; soft electron zone precipitation or low latitude boundary layer precipitation; high-latitude arcs within the above regimes due to field-aligned potential drops; nightside plasma sheet ion precipitation; dayside plasma sheet ion precipitation; diffuse plasma sheet electron precipitation, with medium energy spikes larger than $150 \mathrm{keV}$; discrete auroral arcs embedded within diffuse plasma sheet electron precipitation and storm-time precipitation.

\section{Radiation belt electrons}

The source of precipitating electrons is the electron radiation belt, precipitation is believed to be the main mechanism of electron losses from the Earth's radiation belt (e.g., Horne and Thorne 2003). The electron radiation belt has a structure with maximum relativistic electron intensity around $\mathrm{L} \approx 1.6$ and $\mathrm{L} \approx 4, \mathrm{~L}$ is the McIlwain parameter. The depleted region between the inner and outer electron belts is known as the slot region that is formed due to the electron loss.

The reason for precipitation is violation of the stable motion of the trapped particles, which may be caused by magnetic storms (Horne et al. 2009), lightning discharges (e.g., Voss et al. 1984, 1998; Bučík et al. 2006), earthquakes (Galper et al. 1995; Aleksandrin et al. 2003) or the man-made VLF transmitters on the ground. Schematically, processes leading to precipitation are as follows. All these types of disturbances lead to VLF wave generation. Wave-particle interactions cause both particle acceleration and pitch-angle scattering followed by losses. Thus acceleration and losses are competitive processes leading to dramatic variability of the outer electron belt especially in the $\mathrm{L}>3$ region. Various types of waves interact resonantly with electrons of various energy, therefore the energy spectrum of precipitating electrons varies both from event to event and also during one event.

Electrons do not penetrate into the atmosphere deeper than $\approx 50 \mathrm{~km}$ due to the high ionization and radiation energy losses. However, they generate X-rays via bremsstrahlung. This radiation can reach altitudes of about $20 \mathrm{~km}$ and lower. Balloon and rocket observations of X-ray precipitation were started in the 1950s and 60s (Lazutin 1965), and the results of observations till 1980s are summarized by Laštovička and Križan (2005). More recently, several comprehensive balloon experiments dedicated to X-ray measurements have been made; these will be considered in more detail in Section 2.2.2.

However, much information about electron precipitation has been retrieved from observation of subionospheric radio wave propagation (e.g., Rodger et al. 2007, 2010; Clilverd et al. 2007, 2010a,b) and onboard satellites (e.g., Imhof et al. 1991; Baker et al. 1994; Nakamura et al. 1995, 2000; Blake et al. 1996; Bučík et al. 2006; Horne et al. 2009; Vainio et al. 2009), and references therein, where the precipitating electrons can be identified from the pitch angle distribution and where particle flux dynamics can 
be observed in detail. The electron precipitation covers the energy range from tens of $\mathrm{keV}$ up to several $\mathrm{MeV}$ and lasts from a fraction of a second to several hours.

\section{Auroral electrons}

The best-known auroral features are the northern and southern auroral ovals (Akasofu 1966; Feldstein 1973; Eather 1973). Viewed from space with a global imager, they appear as diffuse, continuous, luminous bands that surround both geomagnetic poles at ionospheric altitudes. The ovals are the result of currents flowing continuously in and out of the ionosphere (Iijima and Potemra 1976a,b). These currents are driven by the continuous interaction between plasma from the Sun (solar wind) and plasma that is bound magnetically to the Earth. The currents flow along geomagnetic field lines, and the ovals coincide with the upward field-aligned current regions where electrons flow into the ionosphere. Upon collisions these electrons are energetic enough to transfer ionospheric atoms and molecules into energetically excited states that may be deexcited by emission of characteristic photons, thus creating the aurora. The global distribution of electrons and ions moving into the ionosphere and creating auroral displays can be well described with statistical models that take into account geomagnetic conditions (Hardy et al. 1985, 1989).

Auroral particles (electrons and ions) integral number flux, integral energy flux, and average energy are specified as a function of corrected geomagnetic latitude (CGL) and magnetic local time (MLT) for a range of magnetospheric conditions, parameterized by either geomagnetic index $\mathrm{K}_{p}$ or by $\mathrm{B}_{z}$ component of the interplanetary magnetic field (IMF) and the solar wind velocity (Vsw). From these statistical auroral flux maps, the number and energy fluxes precipitating over the entire auroral oval can be estimated for periods spanning from weak to strong magnetic activity.

The electron diffuse aurora is scattered mostly by waves, especially by broadband electrostatic waves. On the dayside, where such wave activity is less intense, there can be trapped plasma sheet energy electrons which do not precipitate. Solar wind pressure pulses can lead to the observation of an electron diffuse aurora equatorward of previously existing dayside precipitation, presumably because of induced pitch angle scattering.

As for diffuse aurora, Wing and Newell (1998) showed for ions, and Kletzing et al. (2003) for electrons, that the diffuse precipitation is primarily a kappa distribution (as is true of the source plasma sheet population (Christon et al. 1991)). That is, it is Maxwellian, but with a power law tail at high energies. The primary question for the diffuse aurora is how the loss cone is continually refilled, given the relative shortness of the bounce time (seconds for electrons, minutes or tens of minutes for ions). The answer is better understood for ions, for which pitch angle scattering crossing curved magnetic fields is crucial. This is because the ion gyroradius can be comparable to the radius of curvature of the magnetic field lines. On the nightside, pitch angle scattering occurs crossing the current sheet (Sergeev et al. 1983) and precipitation ceases after the curvature of the field lines is reduced moving earthward. On the dayside, curvature effects associated with the magnetopause and boundary layers can cause ion precipitation (Sergeev et al. 1997).

There have been a number of auroral precipitation models developed in order to better understand the interactions between the geomagnetic field, the solar wind, and the ionosphere, and concomitant impacts on communications (e.g., Brautigam et al. 
1991; Hardy et al. 1991; Lui et al. 2003; Zhang and Paxton 2008; Newell et al. 2010). The OVATION Prime model was developed using energetic particle measurements from the polar-orbiting DMSP (Defense Meteorological Satellite Program) satellites and considers four types of aurorae: two types of discrete electron aurorae (monoenergetic and broadband) and two types of diffuse aurorae (electron and ion) (Newell et al. 2010). The often spectacular discrete aurorae are thin (100 to several $1000 \mathrm{~m}$ ) vertical sheets and rayed arcs which generally run in the east-west direction and which may dance about with changing intensities, colors and shapes during active conditions (Prölss 2004). Discrete aurorae are produced by accelerated particles: the monoenergetic discrete aurora is due to acceleration by quasistatic electric fields, while the broadband discrete aurora is produced by dispersive Alfven waves and has the largest response to geomagnetic activity (Newell et al. 2009). In contrast, diffuse aurorae are broad (100 to $1000 \mathrm{~km}$ wide) patches which sometimes pulse slowly at about $0.1 \mathrm{~Hz}$ (Jones 1974). Although the diffuse electron aurora contains the majority of the auroral energy flux (Newell et al. 2009), its lack of structure and intensity generally makes it less apparent and less visually intriguing than the discrete aurora. The diffuse electron aurora is due to plasma sheet particles in the equatorial magnetosphere that have been scattered into the loss cone (pitch angle diffusion) mostly by chorus waves (Thorne et al. 2010). The ion diffuse aurora is weak and so it is difficult to detect visually (Jones 1974). On the nightside it is primarily due to pitch angle scattering in the equatorial current sheet (Sergeev et al. 1983).

Since most of the visible wavelengths in aurorae are generated through combinations of primary and secondary collisional processes and chemical reactions (Prölss 2004), the emission rates at various visible wavelengths are dependent on oxygennitrogen ratios and/or electron energy spectra. The violet and blue lines are dominated by emissions from ionized molecular nitrogen $\mathrm{N}_{2}^{+}$, the green line at $557.7 \mathrm{~nm}$ and the red lines near $630 \mathrm{~nm}$ by emissions from atomic oxygen $(\mathrm{O})$, and the red lines above $640 \mathrm{~nm}$ by emissions from molecular nitrogen $\left(\mathrm{N}_{2}\right)$. The auroral emissions are also height dependent because particles with higher energies can penetrate deeper into the atmosphere. The green lines are generally emitted at lower altitudes than the oxygen red lines.

During an auroral substorm, the visibility of the aurora increases as the aurora brightens and expands both in latitude and longitude (Prölss 2004). The onset location of auroral activity is usually near 2230 MLT (magnetic local time) and $67^{\circ}$ MLAT (magnetic latitude) and depends on the season and the interplanetary magnetic field (IMF) (Liou et al. 2001). During a substorm, the aurora becomes more dynamic with fluctuating shapes, colors and intensities.

\subsubsection{Types of precipitation}

One type of electron precipitation is related to relativistic electron microbursts, which include electrons with energies above $1 \mathrm{MeV}$ and lasts less than one second duration. They occur preferentially on the night-side (0200-1000 MLT) near the edge of the trapping boundary at $\mathrm{L}=4-6$. Because of this local time dependence, microbursts have been associated with VLF chorus waves (Lorentzen et al. 2001). Microbursts form an important fraction of the precipitation and are able to deplete the radiation belt during days (Thorne et al. 2005).

Long-lasting precipitation events have duration from minutes to hours and occur at $\mathrm{L}=4-7$. They are observed in the late afternoon/dusk sector. They demonstrate 
a fine temporal structure at the scale of $100 \mathrm{~ms}$ to minutes and a variable energy spectrum. These events are often connected with magnetic substorms and usually begin in the pre-midnight sector (1500-2400 MLT), typically showing decreases of around three orders of magnitude in $>2 \mathrm{MeV}$ electron flux within a few hours of the onset, followed by an extended period of low flux. The minute-hour events form the primary loss mechanism for outer zone relativistic electrons (Vainio et al. 2009) and may be produced by electromagnetic ion cyclotron (EMIC) waves (Millan et al. 2002). The energy spectrum of precipitating electrons varies from the main to recovery phase of the geomagnetic storm. Thus, the strongest precipitation of the $>300 \mathrm{keV}$ electrons occurs during the main phase while that of $>1 \mathrm{MeV}$ electrons during the recovery phase. The possible explanation is suggested by Vainio et al. (2009), who also stated that the most significant effect on atmospheric chemistry should be expected in the southern hemisphere poleward of the South Atlantic Anomaly region with a delay of 1-2 days or more with respect to the peak of the geomagnetic storm.

The rate of long-lasting precipitation events correlates with the number of geomagnetic storms, although this is not one-to one relation (Reeves et al. 2003). The population of relativistic electrons is closely related to the occurrence of high speed $(\geq 600 \mathrm{~km} / \mathrm{s})$ solar wind streams and often shows a periodicity of 27 days (Baker and Pulkkinen 1997). Since high speed solar wind can be associated with both coronal mass ejections (CMEs) and the corotating interaction regions (CIR) related to long-lived coronal holes, their occurrence is governed by the 11-year solar cycle. The maximum frequency of energetic electron precipitation (EEP) is observed during the declining phase of the sunspot cycle, when CIRs are most numerous (Bazilevskaya and Svirzhevskaya 1998; Makhmutov et al. 2005). However, very strong relativistic electron events can appear around solar maximum in association with CMEs and the related magnetic clouds interacting with the Earth's magnetosphere.

In addition to magnetic storms, electron precipitation may be caused by groundbased VLF-transmitters (e.g., Datlowe and Imhof 1990), lightning (Voss et al. 1984, 1998) or electromagnetic emission from the ground initiated by seismic activity (Galper et al. 1995; Aleksandrin et al. 2003). Lightning-induced electromagnetic disturbances escape into the magnetosphere and propagate as whistler mode waves, undergo a waveparticle interaction in the radiation belt and lead to pitch-angle scattering of electrons into the bounce loss cone and their precipitation into the atmosphere. Lightninginduced precipitation has been observed as microbursts of electrons with the energy below hundreds $\mathrm{keV}$ and the duration of $\leq 200 \mathrm{~ms}$ (Voss et al. 1984). Their spatial distribution typically correlates with regions of enhanced thunderstorm activity, but it is not studied well because of the complexity of processes involved. Electromagnetic emission can be generated during earthquakes. Emission extends upwards through the ionosphere into the magnetosphere where it propagates as Alfvén waves along the geomagnetic field lines and interacts with trapped particles, causing particle precipitation as a result of pitch-angle redistribution. The precipitation can be observed not only over the earthquake epicenter but at any longitude where a satellite crosses the disturbed L-shell (Aleksandrin et al. 2003).

Liou et al. (2001) investigated the seasonal effects on auroral acceleration and precipitation by analyzing a large number of summer and winter auroral images acquired from the ultraviolet imager (UVI) aboard the Polar spacecraft. On the basis of more than 20000 auroral images in the Lyman-Birge-Hopfield (LBH) bands from Polar UVI observations and on the basis of the numerical modeling results of Germany et al. (1998), they found the following main results: (1) In contrast to nightside auroras, 


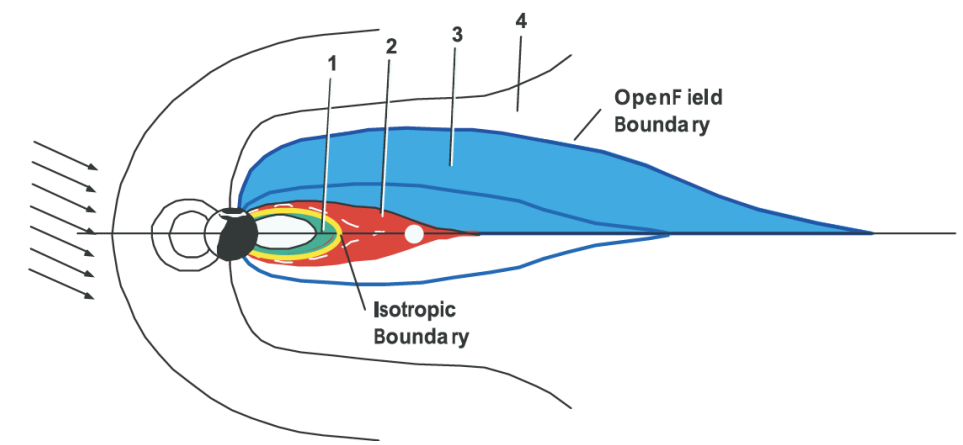

Fig. 20 The magnetospheric boundaries from precipitating particle data. From low latitudes (1) adiabatic, trapped ring current region with particles on dipole-like field lines, minimal precipitation (green), (2) the region of intense proton precipitation (red), (3) the region of diffuse and structured auroras with minimal proton precipitation (blue), and (4) the region of the polar cap. The white spot indicates the region of substorm onset. Figure is reprinted from work of Mende et al. (2003), with permission from Wiley.

which are suppressed in summer, dayside auroras are enhanced and reveal the socalled postnoon auroral bright spots in the sunlit hemisphere (2) precipitating electron energy is higher in winter than in summer; and (3) electron number flux is smaller in winter than in summer. These results indicate that the suppression of nightside aurora in summer is mainly a result of a decrease in the average energies of precipitating electrons, while the enhancement of dayside aurora in summer is mainly a result of an increase in the number flux of precipitating electrons. It is strongly suggested that ionospheric conductance and plasma density in the acceleration regions play an important role in the auroral acceleration mechanism. The day-night asymmetric response of auroras to season suggests a voltage generator for the dayside magnetosphere and a current generator for the nightside magnetosphere.

The magnetospheric proton and electron precipitation boundaries are summarized in Fig. 20. Starting from lower latitudes there is region 1, see Fig. 20, of the adiabatic, trapped ring current particles on dipole-like field lines. These ions are trapped and have no precipitating signatures and cannot be seen in the aurora. At higher latitudes there is the "isotropic boundary" between the adiabatic and stochastic particle motion (Sergeev and Malkov 1988) separating the region of proton precipitation (region 2 in Fig. 20). Further poleward, region 3, see Fig. 20, was found from comparison of the data between the FAST (Fast Auroral Snapshot Explorer) and IMAGE spacecraft (Mende et al. 2003). Poleward of this boundary in region 3, see Fig. 20, there can be weak fluxes of protons, which can only be seen by in situ particle detectors on satellites such as FAST. This region, containing diffuse and structured auroras with minimal proton precipitation, maps into the plasma sheet containing closed field lines (Feldshtein and Galperin 1993). The next region (region 4, see Fig. 20) is the polar cap, which is the region of open field lines, identified usually by the lack of energetic proton and electron precipitation and by the presence of polar rain when the conditions are favorable.

The mean intensity and probability of occurrence of precipitation as a function of magnetic latitude (ML) and magnetic local time (MLT) was established statistically from the analysis of satellite data (Hardy et al. 1987, 1991; Newell et al. 1996). Auroral regions and their boundaries have been extensively classified from DMSP (Defense 
Meteorological Satellite Program) satellite measurements (Newell et al. 1996). The region equatorward (illustrated in blue in Fig. 20) is the plasma sheet, characterized by structured and diffuse electron auroral activity. The region containing intense proton precipitation (illustrated in red in Fig. 20) was not specifically classified by Newell et al. (1996). This region presumably is the result of strongly precipitating magnetospheric protons due to proton isotropization by the highly stretched field lines in the tail. This region is separated by the isotropic boundary (IB) (Sergeev et al. 1993) or the boundary (Newell et al. 1996) from the equatorward ring current region, where there is no significant precipitation.

Newell et al. (2010) used a combination of global EUV images and DMSP particle observations to investigate how various types of aurora evolve over the course of a substorm cycle. A substorm onset results in an increase in all types of auroral activity. The diffuse electron $(80 \%)$ and ion precipitation $(28 \%)$ increase after onset. The authors (Newell et al. 2010) showed, on the basis of a superposed epoch analysis of 4861 substorms, broadband electron acceleration precipitating power jumps $182 \%$ within the first 10-16 min after an onset. This easily surpasses the $79 \%$ increase in monoenergetic aurora, or the diffuse auroral increases. It was also mentioned that the wave auroral power is more concentrated shortly after onset, with relatively little power before onset, and reverting more quickly after the post onset peak. However the diffuse auroral power is increasing until about $50 \mathrm{~min}$ after onset, perhaps as the reconfiguration of the magnetotail to a more dipolar status is gradually completed. By that time broadband acceleration is close to resuming its quite low pre onset values. Although both the number of spectra showing broadband acceleration and the characteristic energy of acceleration increase, it is the latter effect that is most responsible for the jump in precipitating power. The authors (Newell et al. 2010) concretize that the characteristic energy of broadband acceleration rises from about $500 \mathrm{eV}$ to about $1.4 \mathrm{keV}$ over about 10 min (implying electron acceleration up to several $\mathrm{keV}$ to achieve that mean).

\subsubsection{Recent measurements in the atmosphere}

Many medium-scale aurorae were discovered from space because a ground-based observer has to be at the right location at the right time in order to see those features. Some of these features were first described in the 1970s or 1980s using observations from the ISIS 2 (International Satellites for Ionospheric Studies) and Viking satellites. What has changed dramatically over the past years is the almost continuous availability of coincident solar wind data that now allow us to place recent observations into context with the large-scale perspective of the solar wind-magnetosphere-ionosphere interactions. This resulted in statistical investigations of large numbers of events and helped in determining the preferred external conditions that favored or suppressed a particular auroral display.

A limited number of dedicated balloon measurements of the energy spectrum of $\mathrm{X}$-rays generated by precipitating relativistic electrons have been conducted recently (Millan et al. 2002). The first circumpolar balloon flight over Antarctica was performed in 1990 with a hard X-ray spectrometer onboard which detected bremsstrahlung emission extending up to $\approx 300 \mathrm{keV}$ (Smith et al. 1995). In the course of a 9-day long flight six X-ray events were observed. The spectra of precipitating electrons were derived from observation under assumption of uniform emission on the sky.

Foat et al. (1998) observed X-ray bremsstrahlung from relativistic electrons precipitation (REPs) with a balloon-borne Geiger detector at L=5.7 on August 20-th, 1996 
during low geomagnetic activity. Precipitation occurred at 1532-1554 UT, consisted of seven peaks of $60-90$ s duration spaced by $100-200$ s, with quasi-periodic $10-20$ s modulation. The observed modulation may be related to scattering of $\mathrm{MeV}$ electrons by EMIC waves but this requires significantly enhanced plasma density. The precipitating electron spectrum derived from the X-ray spectrum varied in the course of the event but in general was very hard $\left(\mathrm{E}^{-1.6}\right.$, from 100 to $\left.250 \mathrm{keV}\right)$, consistent with a model of bremsstrahlung emission by monoenergetic $1.7 \mathrm{MeV}$ electrons incident at the top of the atmosphere. Foat et al. (1998) stressed that the precipitation mechanism should be highly energy selective because the trapped electron spectrum is steep.

In January 2000 the MAXIS (MeV Auroral X-ray Imaging and Spectroscopy) balloon payload performed an 18-day long flight over Antarctica (Millan et al. 2002). Twenty five events of electron precipitation in the range of $20-1300 \mathrm{keV}$ were observed. The observed precipitation events lasted from $\approx 1$ min to several hours and showed fast temporal modulation in the range 10-140 s. Nine events lasting from minutes to hours were characterized by an extremely flat spectrum $\left(\propto \mathrm{E}^{-2}\right)$, occurred at $\mathrm{L}=3.8-6.7$ but only in the late afternoon/dusk sectors (1430-0000 MLT). This is consistent with scattering by EMIC waves as a precipitation mechanism. The remaining 16 events have a soft spectrum and occurred at all longitudes.

The first quantitative comparison of precipitating and geomagnetically trapped electrons was presented by Millan et al. (2007) based upon MAXIS balloon measurement at L=4.7 on 19-20 January 2000 (Millan et al. 2002). The REP energy spectrum was estimated from observations as exponential with an e-folding energy of $290 \mathrm{keV}$ and a lower energy cut-off of $400 \mathrm{keV}$. The duration of the event implies precipitation extended over at least 3 hours of MLT, assuming a source fixed in local time. Comparison of the precipitation rate with the flux decrease measured at GPS implies that the loss cone flux was only $\approx 1 \%$ of the equatorial flux.

Simultaneous observations of $\mathrm{MeV}$ electron precipitation from multiple balloonborne spectrometers were performed in January 2005 during the MINIS balloon campaign. Six small balloons were launched (4 from Antarctica and 2 from a northern polar station) with the aim to study spatial extent and variation, north-south conjugacy, and evolution as electrons drift over several hours of local time. presented initial results from MINIS focusing on the hard-spectrum electron precipitation observed during the magnetic storm of January 21, 2005 (Dst $=-95$ ). At the beginning of the storm, two southern and one northern balloons observed precipitation although they were widely separated in local time and L shell. Also, at 1844 UT a simultaneous (within $\approx 3 \mathrm{sec}$ ) onset of rise in the high-energy flux occurred at two southern balloons despite them being $660 \mathrm{~km}$ apart. The spectral shape observed at these two southern payloads was similar to the precision of a few percent in a power-law fit from 150 to $600 \mathrm{keV}$, with the indices -2.36 and -2.44 . The derived electron energy spectra for the observed X-ray spectra were most accurately modeled by an exponential electron spectra with the efoldings of $800 \pm 50 \mathrm{keV}$ with one exception when the spectrum was harder, extended to higher energies, and was best fit with the e-folding of $\approx 1.5 \mathrm{MeV}$. The loss rate, averaged over 8 days of flight, of $>500 \mathrm{keV}$ electrons observed by MINIS balloons was 25-50 electrons $/ \mathrm{cm}^{2} / \mathrm{s}$ comparing to the MAXIS average value of electrons $/ \mathrm{cm}^{2} / \mathrm{s}$ for a non-storm-time (Millan et al. 2002, 2007). The MINIS observations show an orderof-magnitude lower average precipitation rate of $>500 \mathrm{keV}$ electrons during this observation period than was found during the January 2000 MAXIS campaign suggesting storm-to-storm variability. Analysis of precipitation spatial distribution is complicated because of balloon drift during the event. 


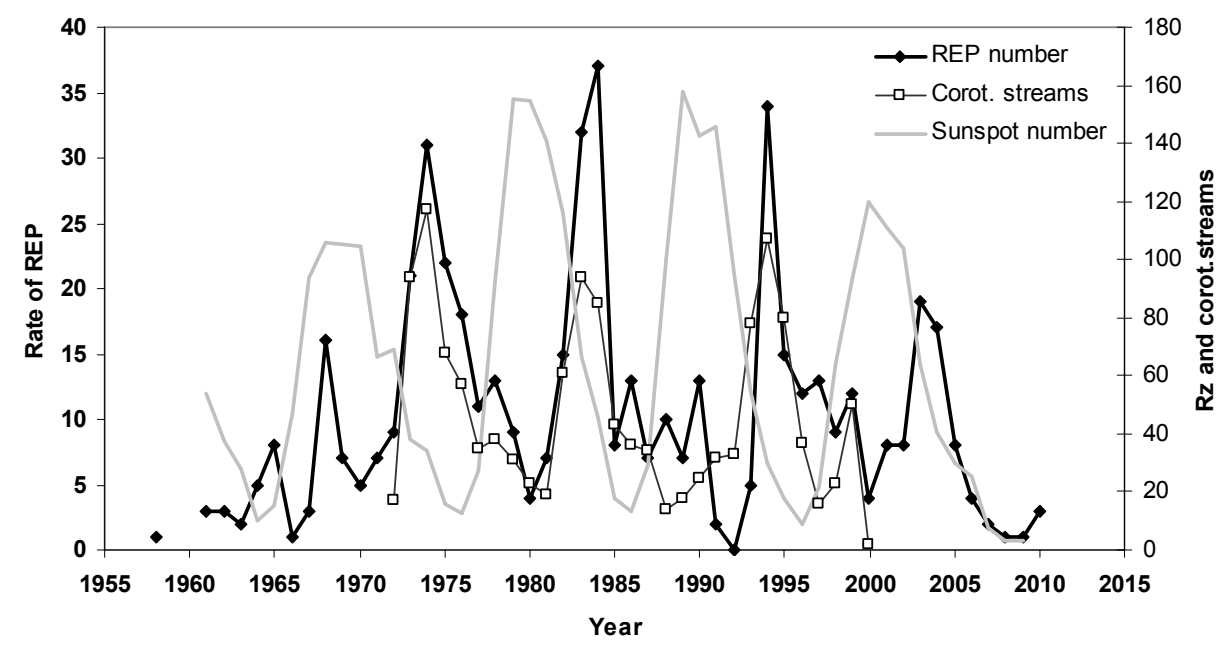

Fig. 21 Yearly numbers of REP recorded in the stratosphere alongside with sunspot numbers and numbers of geomagnetic disturbances associated with corotating solar wind structures (Richardson et al. 2001). It should be noted that some REP before early the 1970s may be omitted.

Long-term regular observations of charged particles in the atmosphere have been performed by the balloon measurements of the Lebedev Physical Institute since 1957 (Charakhchyan 1964). The main goal of the project is a study of charged particle dynamics in the atmosphere. However the X-ray bursts in the atmosphere are also recorded as the balloon-borne detector is sensitive to X-rays although with low efficiency of about 1\% (Bazilevskaya and Svirzhevskaya 1998). Observations are conducted every day at two polar latitudes: Murmansk region $(\mathrm{L}=5.6)$ and Mirny, Antarctica $(\mathrm{L}>10)$. More than 500 events of enhanced X-ray fluxes were detected over Murmansk between 1958-2006, while only 11 events over Mirny were observed.

The rates of X-ray events recorded in the atmosphere exhibit a 27-day variation and seasonal dependence with a maximum in spring and autumn (Makhmutov et al. 2003, 2005). Long-term variation of the X-ray event rates demonstrates an 11-year cycle with maximum values at the descending phase of the sunspot cycle (1973, 1984, 1994 and 2002) (see Fig. 21). Similar temporal behavior is observed for geomagnetic disturbances associated with corotating solar wind structures while the transient CME-related geomagnetic storms occur in phase with solar activity (Richardson et al. 2001). The correlation coefficient between the frequencies of X-ray events observed in the stratosphere during 1972-2000 and geomagnetic storms related to corotating solar wind streams (Richardson et al. 2001) is $0.80 \pm 0.07$. It should be noted that a certain amount of X-ray events occur during solar energetic particle intrusions into the stratosphere (Bazilevskaya and Svirzhevskaya 2002) and are associated with CME-related disturbances. However these events are not numerous. Apparently, CME-related disturbances do not contribute significantly to the temporal profile of the X-ray event rate in the stratosphere that is mostly associated with high-speed solar wind streams. It is known that relativistic electron fluxes in the outer magnetosphere also correlate with high-speed solar wind streams from coronal holes (Baker and Pulkkinen 1997). 
One of the new orbiters missions is The Van Allen Probes, formerly known as the Radiation Belt Storm Probes (RBSP), launched on 30th August, 2012. Two Earth orbiters are dedicated to study of dynamics of Earth's radiation belts and have already published several important results, concerning the behavior of relativistic electrons (Baker et al. 2014).

The problems of REP and related questions concerning the dynamics of the radiation belt are subject of intense discussion in the scientific literature. Summarizing the results of direct X-ray measurements, it is clear that more measurements are required in order to understand the physical mechanisms of precipitation of electrons from the radiation belts. Insufficient observations also mean that the most probable energy spectrum of REP and its variability is not known and the whole energy deposited in the REPs and its spatial and temporal distribution are not clear as yet. Currently, there are significant uncertainties surrounding the precipitating characteristics of medium energy electrons $(>20 \mathrm{keV})$, and even more uncertainties for relativistic electrons. In terms of understanding potential REP and X-ray effects on atmospheric processes, many more observations of these phenomena are required. 


\section{ENERGETIC PARTICLES INFLUENCE ON ATMOSPHERIC PROCESSES}

In the previous Section 2, the nature of energetic particles (EPs) which can precipitate into the atmosphere was discussed.

The next Section 3 will focus on how these particles can affect atmospheric process in the atmosphere. We will consider separately EPP effects on atmospheric ion properties (Section 3.1); gas phase chemistry and the ozone layer (Section 3.2); atmospheric electricity and the global electrical circuit, and cloud effects (Section 3.3).

\subsection{Ions}

\subsubsection{Introduction to atmospheric ions}

Ions exist in the atmosphere since the atmosphere is subject to both electromagnetic and corpuscular ionizing radiation. The existence of the ionosphere and thus free electrons in the atmosphere was originally detected by radio wave propagation experiments, radiowaves are actually reflected by gradients in the electron concentration profile of the ionosphere. At thermospheric altitudes we find positive ions and electrons, in the collisional mesosphere also negative ions appear, and in stratosphere and troposphere we do not find free electrons, since all negative charge is in the form of negative ions. The continuum extreme ultraviolet (EUV) radiation ionizes the upper part of the ionosphere. At ionospheric F region altitudes from $150 \mathrm{~km}$ to $1000 \mathrm{~km}$, the main positive ions are molecular nitric oxide and atomic oxygen ions while above $\mathrm{F}$ region the ions are lighter helium and hydrogen ions. In the E region, at $100 \mathrm{~km}$ to $150 \mathrm{~km}$ altitude, ionization is caused by X-rays, Lyman beta, EUV continuum, as well as precipitating electrons and protons (Ferguson et al. 1979). The main ions are molecular oxygen and nitric oxide positive ions. In the D region from $60 \mathrm{~km}$ to $100 \mathrm{~km}$ ionizing agents are $\mathrm{X}$-rays, Lyman alpha, galactic cosmic rays (GCRs), energetic electrons and protons and at night the scattered radiation. Main ions in the upper D region are the same as in $\mathrm{E}$ region, but at lower altitudes we have formation of heavier positive cluster ions, appearance of negative ions, as well as negative cluster ions. Below D region ionization by GCRs dominates and the ions are clusters of positive and negative ions. Subsequent ion chemistry, charge and ion charge exchange reactions, electron attachment, electron-ion and ion-ion recombination, dissociative recombination as well as electron detachment further participate in determining the chemical composition ionized air. Although ionization degree is generally very low, it turns out that ion chemistry may significantly affect the concentrations of chemically active minor neutral constituents. Therefore in detailed investigations one has to consider coupled neutral and ion chemistry. Photochemistry turns out to be important, lifetimes of constituents may vary significantly between night and day. Also aerosols do play a role at various altitudes, both in determining the charge balance and bringing in surface and heterogeneous chemistry (Ferguson et al. 1979). Both primary and secondary ionization products have important atmospheric effects. They influence atmospheric electrical properties, radio wave propagation, trace gas formation, and aerosol particle formation. In addition, atmospheric ions are also important by serving as powerful probes for the detection of certain important atmospheric trace gases. 
This section will provide an introduction to atmospheric ions, their composition, and vertical profile throughout the atmosphere. It will also summarize a potential mechanism by which atmospheric ions may influence cloud formation, through ion induced aerosol formation, see Enghoff and Svensmark (2008) for a full review.

\subsubsection{Sources and sinks of atmospheric electrically charged species}

Atmospheric ionization sources (shown schematically in Fig. 1), involve different types of energetic photons and energetic particles. These enter the atmosphere from deep space (solar EUV and X-rays, GCRs, SEPs/GLEs and energetic electrons precipitation $(\mathrm{EEP})$ ), or are formed in the atmosphere (electric discharges), or stem from ground level sources (mostly the radioactive noble gas radon). Removal of atmospheric ions and free electrons proceeds preferably via neutralization (Harrison and Carslaw 2003) by mutual recombination of positive ions and negative charged species in the gas-phase. Only in air masses having a sufficiently large aerosol load, charged species may attach (Harrison and Carslaw 2003) preferably to aerosol particles rather than experience recombination in the gas-phase.

Recombination of charged species in the gas-phase is strongly influenced by the charged species nature. The rate coefficient for positive ion recombination with free electrons increases very strongly with increasing positive ion complexity, spanning 6 orders of magnitude. For atomic ions it is only about $10^{-11} \mathrm{~cm}^{3} \mathrm{~s}^{-1}$, while for complex cluster ions it is about $10^{-5} \mathrm{~cm}^{3} \mathrm{~s}^{-1}$, see Arnold and Krankowsky (1977); Arijs and Brasseur (1986); Harrison (2000). By contrast, recombination of positive ions with negative ions depends only relatively weakly on the ion nature, but varies markedly with atmospheric gas pressure. Therefore, understanding of atmospheric charged species sinks and concentrations requires detailed knowledge of the recombining charged species nature and chemical evolution. In most atmospheric conditions, charged species concentrations $\mathrm{n}_{+}$and $\mathrm{n}_{-}$are determined by a steady-state of ionization and recombination: $Q=\alpha \cdot n_{+} \cdot n_{-}$, where $\mathrm{Q}$ is the ionization rate and $\alpha$ is the effective recombination coefficient. Due to charge neutrality one obtains $n_{+}=n_{-}=\sqrt{(Q / \alpha)}$.

An atmospheric altitude region where ionization sources and sinks of electrically charged species are particularly complex and variable extends between about 60 and $100 \mathrm{~km}$ (lower ionosphere). In this very cold region, ionization by energetic particles is important and ion induced aerosol formation may take place. Due to the influence of energetic particles on ionization, the processes of that region will be discussed in greater detail. Figure 22 depicts daytime and nighttime ionization sources for that altitude region. At daytime, around $85 \mathrm{~km}$, NO ionization by Ly-alpha radiation, ionization of excited metastable $\mathrm{O}_{2}$ ionization by EUV, and ionization of air by energetic X-rays are the mayor ioniozation sources. At night, ionization by energetic electron precipitation (EEP) from the Earth's radiation belts, NO ionization by Ly-alpha radiation and ionization by X-rays from the astronomical source Scorpio XR1 are operative in the lower ionosphere. Also in that region and in the upper stratosphere, dramatic transient ionization increases occur during sporadic SEP events, mostly between about 100 and $30 \mathrm{~km}$. Figure 23 depicts ionization rates for a major SEP/GLE event in August 1972. Normally at altitudes from the mid-thermosphere up into $90 \mathrm{~km}$, there is also a contribution to ionization from auroral electrons. The flux of these particles varies greatly with time, and can be enhanced by several orders of magnitude during strong storms. During such events ionization rates in the upper atmosphere are significantly 

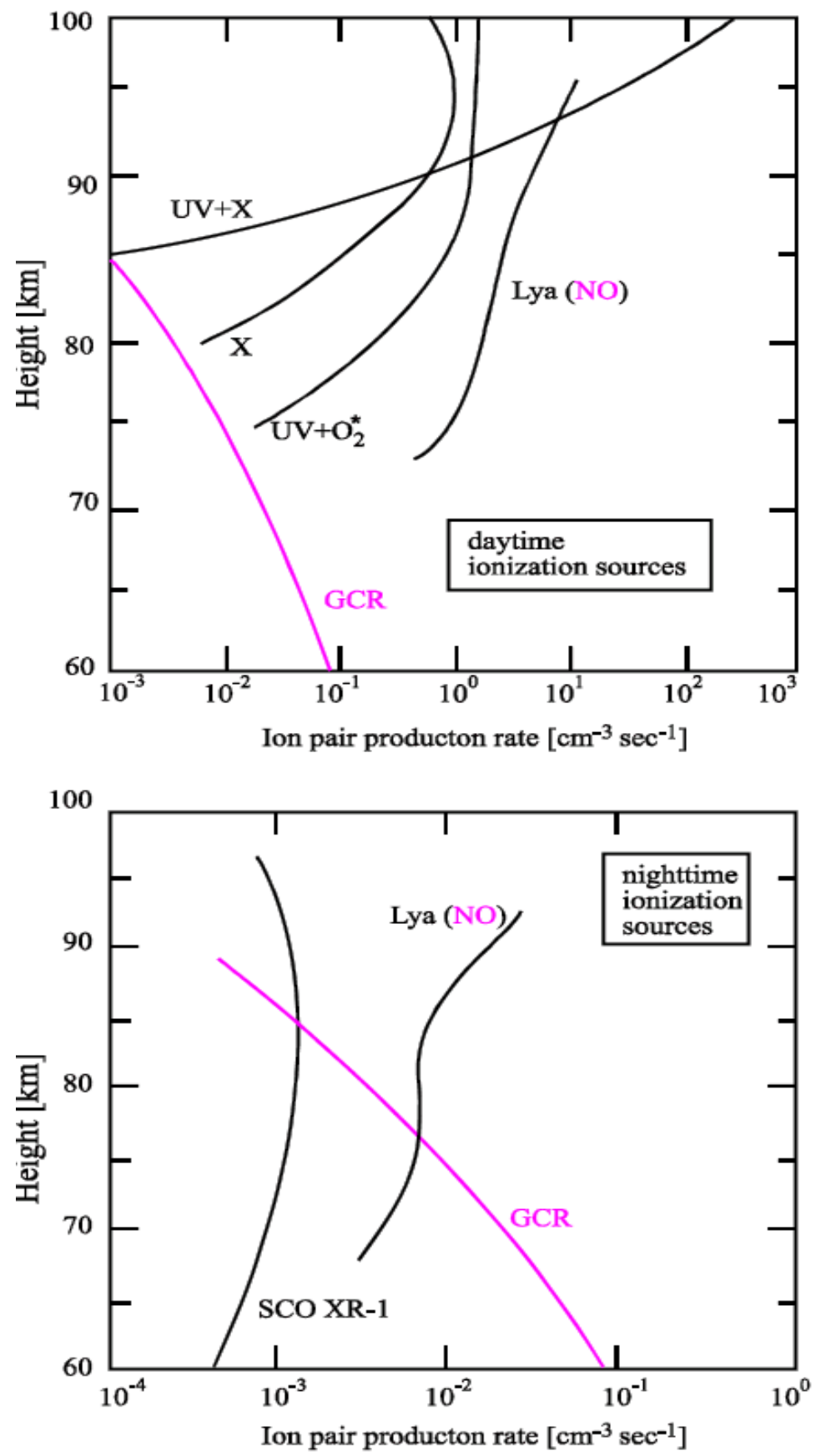

Fig. 22 Lower ionosphere ionization sources for daytime (top) and nighttime (bottom). Modified after (Arnold and Krankowsky 1977). 


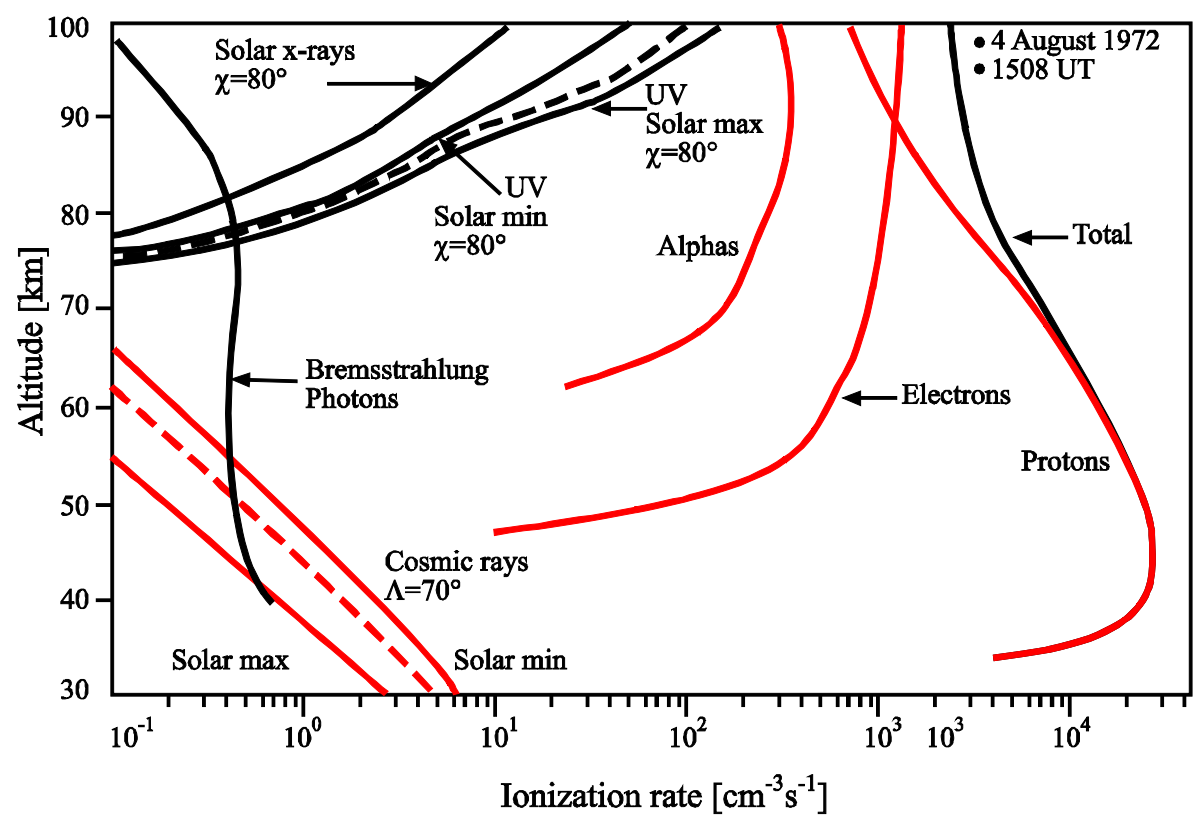

Fig. 23 Ionization rates during a major SEP event on 4th August 1972. Also shown for comparison are rates of ionization by solar UV and X rays as well as ionization by GCRs for a geomagnetic latitude $70^{\circ}$ and for minimum and maximum conditions of the 11 years solar sunspot cycle. Modified from Chapter 7 of Brasseur and Solomon (2005).

enhanced, for example, at 70-150 km. However according to current knowledge, see Figure 1, the ionization rate from electrons vary over nearly four orders of magnitude.

\subsubsection{Ion composition: vertical structure and observations}

The measured total concentration and composition of atmospheric charged species varies markedly with altitude. This is illustrated by the schematic representation shown in Fig. 24. Total daytime concentrations vary from about $4 \cdot 10^{2}$ to almost $1 \cdot 10^{6} \mathrm{~cm}^{-3}$. The maximum total concentration is observed at $200-300 \mathrm{~km}$ altitude, in the ionospheric F-region. The minimum concentration is observed at about $60 \mathrm{~km}$, at the bottom of the ionospheric D-region. Above about $60 \mathrm{~km}$, total concentrations are larger during the daytime, compared to nighttime, since daytime ionization by solar EUV and $\mathrm{X}$-rays is much stronger. Below $60 \mathrm{~km}$, day and night total concentrations are equal, since the dominant ionization source (GCRs) has no diurnal variation (Bazilevskaya et al. 2008). Positive charged species are atomic ions, molecular ions, and cluster ions. Negative charged species are mostly free electrons (above about $70-80 \mathrm{~km}$ ) and negative ions below these heights.

In the ionosphere, free electrons are present in concentrations sufficiently large to influence radio waves. Early ionospheric research probed the ionosphere using radio waves (mostly reflection and absorption). Therefore, the ionosphere may also be named the electron-sphere. It extends from about $60 \mathrm{~km}$ to about $500 \mathrm{~km}$ and is divided into three major sub-layers (Rishbeth and Garriott 1969), the F-layer (about 200 - 500 


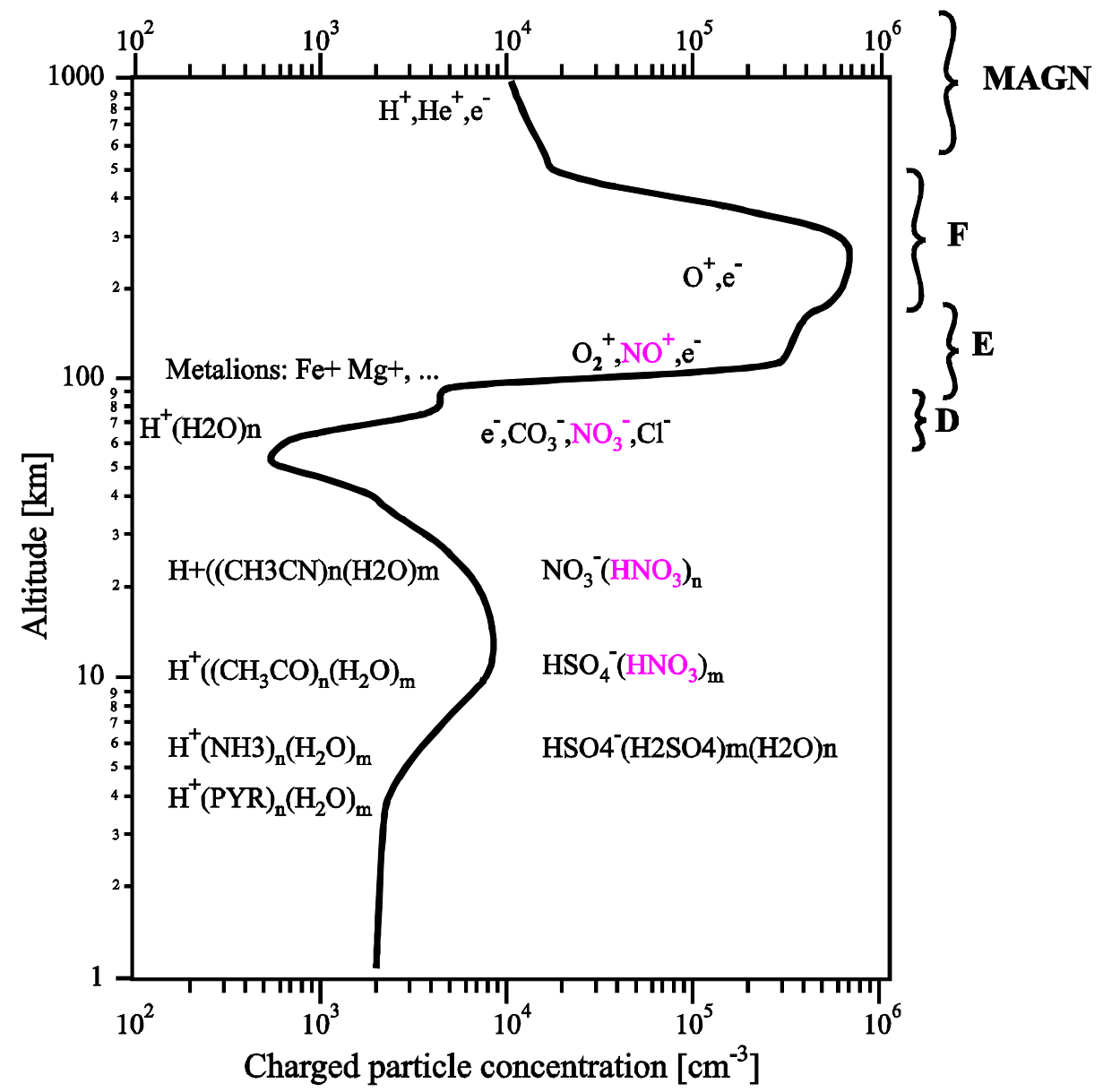

Fig. 24 Schematic representation of total concentration and major ion species in the daytime free atmosphere. Also indicated are ionospheric layers and their names (D, E, F layers and Magn = magnetosphere layer). Highlighted in red are ions whose electrically neutral molecular precursors or attached molecules are influenced by energetic particle radiations. Figure is modified from Ferguson and Arnold (1981).

$\mathrm{km}$ ), E-layer (about $85-200 \mathrm{~km}$ ), and the D-layer (about $60-85 \mathrm{~km}$ ). Below about 70 (day)-80 (night) km, the free electric charges are mostly positive and negative ions, but free electrons are still present. Radio wave absorption occurs in the D-region where the atmospheric gas density is already sufficiently large to cause free electrons, oscillating in the radio wave field, to experience collisions with atmospheric gas molecules.

Next, details of the ion composition will be discussed, starting at the top of the atmosphere and then working downwards in altitude.

At altitudes above about $400 \mathrm{~km}$, where hydrogen and helium are the most abundant gases, the dominant positive ions are the light atomic ions $\mathrm{H}^{+}$and $\mathrm{He}^{+}$. At heights between about 150 and $400 \mathrm{~km}$ the dominant positive ion is $\mathrm{O}^{+}$, reflecting the fact that the dominant gas is atomic oxygen (Ferguson and Arnold 1981). At heights 
between about 150 and $85 \mathrm{~km}$, during daytime and in most situations, the most abundant positive ions are the molecular ions $\mathrm{O}_{2}{ }^{+}$and $\mathrm{NO}^{+}$. While $\mathrm{O}_{2}{ }^{+}$is formed by ionization of the major atmospheric gas $\mathrm{O}_{2}, \mathrm{NO}^{+}$formation involves the atmospheric trace gas $\mathrm{NO}$ and ion molecule reactions of $\mathrm{N}_{2}^{+}$and $\mathrm{N}^{+}$, see Section 3.2 for further details. In addition, also ionized metal atom ions (mostly $\mathrm{Fe}^{+}$and $\mathrm{Mg}^{+}$) are present, and may occasionally be prominent even during daytime. By contrast at nighttime, metal atom ions dominate. At heights below about $85 \mathrm{~km}$, the positive ions are mostly cluster ions, of which water cluster ions of the type $\mathrm{H}^{+}\left(\mathrm{H}_{2} \mathrm{O}\right)_{n}$ are most abundant. At heights below about $70 \mathrm{~km}$ (daytime) and $80 \mathrm{~km}$ (nighttime) negative ions are present and become more abundant than free electrons. These heights define the bottom of the electron-sphere (Ferguson and Arnold 1981).

The presence of major ion species $\left(\mathrm{NO}^{+}\right.$, metal atom ions, cluster ions, negative ions), which cannot be formed from major atmospheric gases (like $\mathrm{O}_{2}^{+}, \mathrm{N}_{2}^{+}, \mathrm{O}^{+}$, and $\mathrm{N}^{+}$) clearly indicates the important role of ion chemistry.

Some more information on observations/formations of primary ions in the mesosphere/low thermosphere can be found in the review paper (Sinnhuber et al. 2012).

The mesosphere extends on average from about 50 to $85 \mathrm{~km}$, although the exact upper and lower boundaries vary with latitude and season. It contains the lowermost ionospheric layer, the so called ionospheric D-region (about $60-85 \mathrm{~km}$ ), which has been discussed already in the above section dealing with the ionosphere. The lower mesosphere free charges are positive and negative ions. Their concentrations range from about $5 \cdot 10^{2}$ to $1 \cdot 10^{3} \mathrm{~cm}^{-3}$ for each charge sign and increase with decreasing height (Ferguson and Arnold 1981). The dominant source of ionization is, in most conditions, galactic cosmic rays (GCRs) and there is also a contribution from solar protons during SEP/GLE events (Bazilevskaya et al. 2008). In addition, photoionization by Lyman-alpha occurs above $70 \mathrm{~km}$, as well as ionization from relativistic electrons. The dominant sinks of positive and negative ions are mutual ion-ion recombination.

Using a rocket borne dropsonde-ion mass spectrometer, delveloped at MPIK-Heidelberg, ion composition data were obtained at altitudes between 35 and $53 \mathrm{~km}$. The positive ion species detected in the lowermost mesosphere (above $50 \mathrm{~km}$ ) were mostly $\mathrm{H}^{+}\left(\mathrm{H}_{2} \mathrm{O}\right)_{n}$ and the negative ion species are consistent with $\mathrm{NO}_{3}^{-}$and $\mathrm{NO}_{3}^{-} \mathrm{HCl}$ (Arnold 1980b,a, 1981, 1982).

The stratosphere extends from about 10 to $50 \mathrm{~km}$ on average. In the stratosphere, free charges are positive and negative cluster ions. Total ion concentrations range from about $5 \cdot 10^{2}$ to $5 \cdot 10^{3} \mathrm{~cm}^{-3}$ (for each sign). They increase with decreasing height, reaching a maximum of almost $10^{4}$ per $\mathrm{cm}^{3}$ at about $15 \mathrm{~km}$. In the stratosphere, the dominant ionization source is galactic cosmic rays (GCRs), leading to free electrons and the primary positive ions $\mathrm{N}_{2}^{+}$(molecular nitrogen ions), $\mathrm{O}_{2}^{+}$(molecular oxygen ions), $\mathrm{N}^{+}$ (atomic nitrogen ions), and $\mathrm{O}^{+}$(atomic oxygen ions).

The major stratospheric ion sink is ion-ion recombination, whose rate coefficient varies only little with the chemical nature of the recombining ions. The ion lifetime with respect to ion-ion recombination varies with altitude, from about $10^{2}$ to $10^{4}$ seconds. During this time span stratospheric ions undergo a chemical evolution involving numerous ion molecule reactions with certain stratospheric trace gases.

Above heights of 33-30 km, the dominant ions are acidic cluster ions of the type $\mathrm{NO}_{3}^{-}\left(\mathrm{HNO}_{3}\right)_{n}$ and below these heights acidic cluster ions of the type $\mathrm{HSO}_{4}^{-}\left(\mathrm{H}_{2} \mathrm{SO}_{4}\right)_{a}\left(\mathrm{HNO}_{3}\right)_{n}$ become prominent. The transition occurs in a relatively narrow layer, extending from about $30-33 \mathrm{~km}$. The acidic cluster ions are formed from precursor ions by ion molecule reactions involving the atmospheric acidic trace gases nitric acid $\left(\mathrm{HNO}_{3}\right)$ and sulfu- 


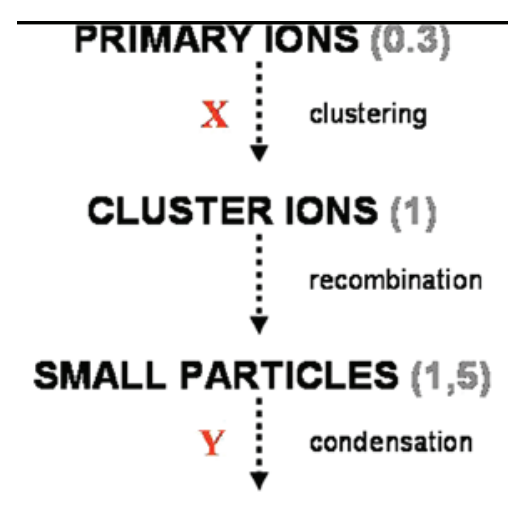

GROWN PARTICLES $(>30)$

Fig. 25 Simplified representation of atmospheric ion-induced particle formation, from particle growth through to CCN. Numbers denote approximate diameters (in $\mathrm{nm}$ ) of ions and particles. $\mathrm{X}$ denotes nucleating gas molecules and Y denotes condensing gas molecules (Arnold 2008).

ric acid $\left(\mathrm{H}_{2} \mathrm{SO}_{4}\right)$. The abrupt transition is due to the steep decrease of atmospheric gas-phase $\mathrm{H}_{2} \mathrm{SO}_{4}$ at these heights (see below).

In the troposphere, extending from the surface to about $6-18 \mathrm{~km}$ (depending on latitude and season), the dominant charged species are positive and negative cluster ions. However, in polluted tropospheric air masses with large total aerosol surface area concentrations, ion attachment to aerosol particles may become appreciable. In the free troposphere, total ion concentrations mostly are around 2000 ions per $\mathrm{cm}^{3}$. The dominant ionization source is GCRs. Over the continents at heights below about $1 \mathrm{~km}$, ionization by the radioactive noble gas radon becomes dominant (Harrison and Carslaw 2003). Radon is released from continental sources, but due to its rather limited lifetime (half life against radioactive decay: about 3.8 days), influences preferably the continents and continental outflow regions over the oceans.

At ground-level, ion composition measurements were originally been made by the Georgia Institute of Technology group of Eisele (Eisele 1989) in a few selected locations. More recently, such measurements have also been made by the University of Helsinki group (Hirsikko et al. 2011).

Ion composition measurements have been made in the free troposphere from aircraft by several groups including Ziereis and Arnold (1986) and at the surface by Eisele (1989) and Hirsikko et al. (2011), The chemical nature of ions present in ground-level in regions, where air is ionized preferably by cosmic rays (remote oceanic regions), is not well known. Hardly any measurements have so far been reported.

\subsubsection{Ion induced aerosol formation}

Atmospheric aerosol particle formation by gas to particle conversion proceeds via nucleation of certain trace gas molecules X (Fig. 25). Nucleation leads to a so called critical molecular cluster, which is just stable in the given atmospheric conditions and which represents already a stable aerosol particle. For a critical cluster the rate of attachment of nucleating molecules equals the rate of thermal detachment of clustered 
molecules. The size of a critical cluster is determined mostly by atmospheric temperature and volatility as well as partial pressure of the nucleating gas-phase molecules $\mathrm{X}$. As temperature decreases, thermal detachment of clustered molecules X from the cluster decreases strongly. As the partial pressure of clustering gas-phase molecules $\mathrm{X}$ increases, the clustering rate increases. Therefore, the diameter of a critical cluster decreases with decreasing temperature and increasing partial pressure of X. In the atmosphere, an important if not the most important, nucleating gas $\mathrm{X}$ is sulfuric acid $\left(\mathrm{H}_{2} \mathrm{SO}_{4}\right)$. It undergoes binary $\left(\mathrm{H}_{2} \mathrm{SO}_{4} / \mathrm{H}_{2} \mathrm{O}\right)$ nucleation, leading to supercooled liquid $\mathrm{H}_{2} \mathrm{SO}_{4} / \mathrm{H}_{2} \mathrm{O}$ aerosol droplets whose $\mathrm{H}_{2} \mathrm{O}$ mass fraction increases with increasing relative humidity $(\mathrm{RH})$. At low temperatures, prevailing in the upper troposphere, a critical $\mathrm{H}_{2} \mathrm{SO}_{4} / \mathrm{H}_{2} \mathrm{O}$ cluster may contain only a few $\mathrm{H}_{2} \mathrm{SO}_{4}$ molecules and may have a diameter of only about $1.5 \mathrm{~nm}$.

By contrast to $\mathrm{H}_{2} \mathrm{SO}_{4}, \mathrm{SO}_{2}$ is much less sticky and therefore can experience longrange transport in the atmosphere. At present, sources of $\mathrm{SO}_{2}$ are mostly fossil fuel combustion, volcanoes, and atmospheric conversion of oceanic plankton generated sulfurbearing gases.

Sulfuric acid nucleation may also involve additional atmospheric clustering molecules possessing much larger proton affinities than $\mathrm{H}_{2} \mathrm{O}$. Examples are ammonia $\left(\mathrm{NH}_{3}\right)$ and amines. However, these gases do not seem to be sufficiently abundant, in the free troposphere and in remote regions of the planetary boundary layer (Ziereis and Arnold 1986) ions may facilitate nucleation. If the molecular cluster carries one net electric charge, it will also become more stable against thermal detachment of clustered molecules X. In addition, the net charge also increases the rate at which molecules, particularly polar molecules, collide with the cluster. Hence, a net charge has an influence somewhat resembling the combined action of decreasing the temperature and increasing the number concentration of clustering gas-phase molecules. Both charge effects are mostly due to charge-dipole interaction forces and tend to increase the nucleation rate. Therefore, ion induced nucleation can become efficient in atmospheric conditions where nucleation via electrically neutral molecular clusters is just not yet efficient, particularly when the temperature is somewhat too high or when the concentration of the nucleating gas molecules $\mathrm{X}$ is somewhat too low for neutral nucleation. However, in typical atmospheric conditions, the rate of ion induced nucleation cannot exceed the ionization rate.

Also, ion induced nucleation is limited by the limited ion lifetime, which in most conditions is limited by charge recombination. During its limited lifetime, an ion must collide with a sufficiently large number of clustering gas-molecules X. For example in the upper troposphere, the ion lifetime against ion-ion recombination is about 200 seconds (Harrison and Carslaw 2003), which requires a gas-phase $\mathrm{H}_{2} \mathrm{SO}_{4}$ number concentration of about $2.5 \cdot 10^{6} \mathrm{~cm}^{-3}$ for an ion to collide during its lifetime with one $\mathrm{H}_{2} \mathrm{SO}_{4}$ molecule. In the upper troposphere, gas-phase $\mathrm{H}_{2} \mathrm{SO}_{4}$ concentrations of up to about $6 \cdot 10^{6} \mathrm{~cm}^{-3}$ have been measured. Hence, several $\mathrm{H}_{2} \mathrm{SO}_{4}$ molecules may cluster to an ion before it recombines with an oppositely charged ion (Arnold 2008). Atmospheric ions may also promote growth of newly formed and still small molecular clusters. Ions may grow, during their limited lifetime, to sizes sufficiently large to allow the neutral molecular cluster formed by their mutual recombination, to be larger than the critical size of a molecular cluster. An ion may also attach to a stable neutral cluster and thereby increase the rate of collision of clustering molecules with the cluster. Furthermore, ion attachment to small aerosol particles may increase coagulation of aerosol particles carrying opposite charges. An obvious and perhaps most direct way to obtain insights into 
atmospheric ion induced nucleation are measurements of the atmospheric ion composition. Additional insights can be obtained from laboratory measurements, atmospheric measurements of nucleating gases, and model simulations. Regarding atmospheric ion composition measurements, large cluster ions may serve as fingerprints of ion induced nucleation. At least, the presence of large cluster ions should mark atmospheric layers or air masses, which are primed for ion induced nucleation. In the following section, the search for large cluster ions using airborne ion mass spectrometers will be reported, in the sequence mesosphere, stratosphere, and free troposphere.

The largest atmospheric cluster ions have been measured in the coldest atmospheric layer, the summer polar mesopause layer. There, temperatures may fall to about $120 \mathrm{~K}$. Also, $\mathrm{H}_{2} \mathrm{O}$ is relatively abundant, as was originally found by rocket borne mass spectrometer measurements (Arnold and Krankowsky 1977). The large cluster ions (Eichkorn et al. 2002), were of the type $\mathrm{H}^{+}\left(\mathrm{H}_{2} \mathrm{O}\right)_{n}$ with $n$ up to 21 .

In reality, $\mathrm{n}$ may even be larger since, in the mass spectrometric measurement, some $\mathrm{H}_{2} \mathrm{O}$ molecules may detach from the cluster ion, during ion sampling by electric fields, due to electric field induced energetic collisions.

It has been suggested that these observed large $\mathrm{H}^{+}\left(\mathrm{H}_{2} \mathrm{O}\right)_{n}$ cluster ions may induce formation of aerosol particles (Arnold 1980a). These may contribute to the formation of noctilucent clouds, see Section 3.4.1 and (Arnold 1980a), which are composed of $\mathrm{H}_{2} \mathrm{O}$. However, at the cold summer mesopause, water vapour may condense on meteor smoke particles (Curtius et al. 2005).

Water vapor condensation on meteor smoke particles may prevent the built up of sufficiently large water vapour supersaturations required for ion induced nucleation. Therefore, at the cold mesopause, ion induced nucleation may take place preferably in air masses experiencing sufficiently rapid cooling induced by atmospheric waves. In such situations, the large water vapour supersaturation, required for ion induced nucleation, may build up.

In the upper stratosphere, large negative cluster ions also have been detected (Henschen and Arnold 1981; Arnold 1981, 1982; Arnold et al. 1982). These are most abundant and largest in a layer at about $30-40 \mathrm{~km}$ altitude. Large cluster ions of the type $\mathrm{HSO}_{4}{ }^{-}\left(\mathrm{H}_{2} \mathrm{SO}_{4}\right)_{a}$ ( $a$ is the number of sulphuric acid molecules) have been detected in an aged volcanic plume at about $25 \mathrm{~km}$ altitude (Arnold et al. 1982; Arnold and Buehrke 1983; Arnold et al. 1990).

In the upper troposphere, large cluster ions have also been detected (Eichkorn et al. 2002). Most likely, these ions are cluster ions containing $\mathrm{H}_{2} \mathrm{SO}_{4}$ and $\mathrm{H}_{2} \mathrm{O}$ molecules (Arnold 2008), formed in air masses containing elevated $\mathrm{SO}_{2}$ concentrations. Figure 26 depicts large cluster ion data obtained by an aircraft-based large ion-mass spectrometer (LIOMAS) in the upper troposphere (Eichkorn et al. 2002; Arnold 2008). Plotted is a time-series of LIOMAS ion composition data in cloud-free upper troposphere air during one aircraft flight (Arnold 2008). While the aircraft was cruising mostly around $8000 \mathrm{~m}$ altitude it intercepted a large air mass containing large negative and positive cluster ions with mass numbers larger than $600 \mathrm{amu}$. Negative and positive ions behave rather similarly which suggests that one or several trace gases $\mathrm{X}$ are present which cluster to negative and positive ions. From the measured ions the concentrations of $\mathrm{X}$ were inferred. These range between $1-4 \cdot 10^{6} \mathrm{~cm}^{-3}$ which is very similar to the gaseous $\mathrm{H}_{2} \mathrm{SO}_{4}$ concentrations measured in the upper troposphere around $8000 \mathrm{~m}$ altitude. This suggests at least that $\mathrm{X}$ is not more abundant than gaseous $\mathrm{H}_{2} \mathrm{SO}_{4}$ which implies that most likely $\mathrm{X}$ can be identified as $\mathrm{H}_{2} \mathrm{SO}_{4}$. These measurements demonstrate that in the cold upper troposphere GCR mediated ion induced nucleation can be very effi- 


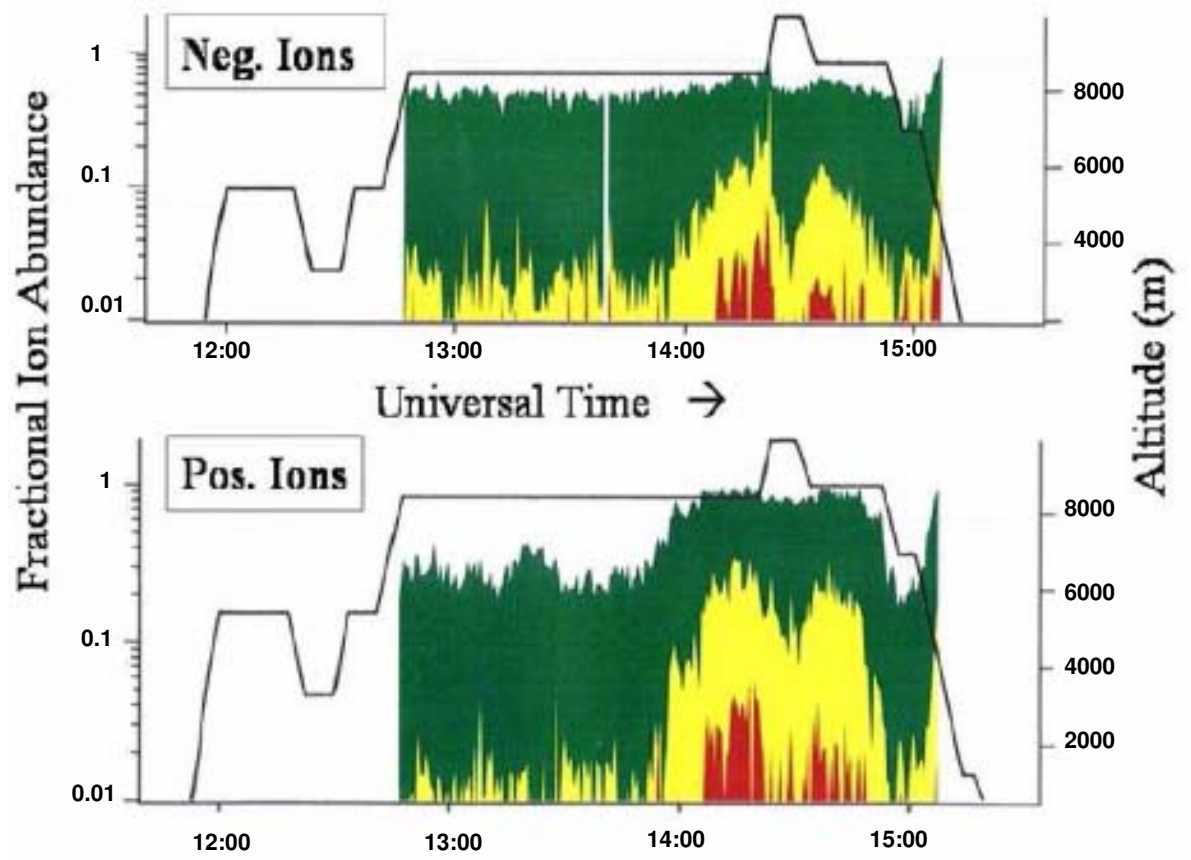

Fig. 26 Fractional abundances of negative ions (upper panel) and positive ions (lower panel) with mass numbers larger than 200 (green), 400 (yellow), and 600 (red) amu measured during a Central European aircraft campaign. Also given is the flight altitude profile (Arnold 2008).

cient. Homogeneous nucleation is also efficient, however and circumstances may exist in which it becomes even more efficient than ion induced nucleation. Charged nucleation in the upper troposphere is also supported by the observations of Lee et al. (2003); Laakso et al. (2004).

\subsubsection{Laboratory investigations of ion induced nucleation}

Laboratory investigations of $\mathrm{H}_{2} \mathrm{SO}_{4} / \mathrm{H}_{2} \mathrm{O}$ nucleation with unambiguous identification of the chemical nature of the involved ion species have been reported by several investigators and are described in Arnold et al. (2000); Lovejoy et al. (2004). For a review of recent laboratory based studies on ion induced nucleation, see Kazil et al. (2008); Enghoff and Svensmark (2008).

More recently, laboratory measurements of ion induced nucleation were extended by the CLOUD project at CERN (Kirkby et al. 2011) and the SKY experiment of the Copenhagen group (Enghoff et al. 2011). CERN extended ion induced nucleation investigations to ternary systems $\mathrm{H}_{2} \mathrm{SO}_{4} / \mathrm{H}_{2} \mathrm{O} / \mathrm{B}$, where $\mathrm{B}$ represents the high proton affinity molecule $\mathrm{NH}_{3}$ (Kirkby et al. 2011). These data suggest that, in the planetary boundary layer in most atmospheric situations, ion induced nucleation is most likely 
unimportant. Instead, nucleation proceeds by a ternary mechanism involving a large proton affinity species like $\mathrm{NH}_{3}$ or dimethyl amine, which is efficient even without a net electric charge. The Copenhagen group extended investigations further by conducting underground measurements to shield the nucleation chamber from GCRs.

\subsubsection{Model simulations of atmospheric ion induced nucleation}

Building on atmospheric measurements of ion species and nucleating trace gases, laboratory data of ion induced nucleation model simulations have been made of ion induced nucleation in the mesosphere, stratosphere, and troposphere. Recently, attempts have been made to incorporate tropospheric ion induced nucleation into a global climate model (Kazil et al. 2006; Pierce and Adams 2009; Kazil et al. 2012). These model studies show that although changes in cloud reflectivity occur from cloud condensation nuclei generated by ion induced nucleation, they are generally too small to play a significant role in current climate change. These issues are discussed in more detail in Section 3.4.3.

\subsubsection{Growth of critical molecular clusters to CCN size}

Critical clusters formed by nucleation may grow sufficiently to become water vapor condensation nuclei (often termed cloud condensation nuclei (CCN). However, in most atmospheric conditions, critical clusters have diameters of only about $1 \mathrm{~nm}$, while a $\mathrm{CCN}$ has a diameter of at least about $70 \mathrm{~nm}$. This means that very substantial growth and mutual coagulation of new aerosol particles is required. In turn, this requires sufficiently large concentrations of condensing trace gases Y. Condensing gases Y can be somewhat more volatile than nucleating gases X. If $\mathrm{Y}$ concentrations are sufficiently large to account for the required growth, the rate of neutral nucleation would be much larger than the rate of ion induced nucleation, which is limited by the ionization rate. At least in the free atmosphere, sufficiently large concentrations of $\mathrm{Y}$ gases have so far not been observed, despite intense searches (e.g., Arnold 2008). Therefore it seems that there exists a severe bottleneck for free atmosphere ion induced CCN formation. 
3.2 Effects of energetic particles on neutral gas-phase chemistry and the ozone layer

\subsubsection{Introduction to EPPs effect on chemistry of the atmosphere}

Ionization of the atmosphere by different energetic precipitating particles (EPPs) described in Section 2 can directly affect chemical composition with implications for further changes in dynamics and climate. Long term satellite observations and physically based parameterizations of the EPP effects on atmospheric chemistry have been available since 1980s and the influence of EPPs on gas chemistry has been investigated using both observational data analysis and numerical experiments with models of different complexity. However, these studies were traditionally aimed at the middle atmosphere and addressed short term events such as SEPs to be consistent with Section 2.1.4 and geomagnetic storms which are characterized by high signal to noise ratio facilitating the clear detection of the response. Therefore EP effects have not been taken into account for the modelling of either ozone (WMO 2011) or climate (IPCC $2007,2013)$ change in the past and future. In these assessments the anthropogenic factors as well as volcanic eruptions and solar ultraviolet irradiance variability have been considered as the major drivers of climate and ozone changes. It is only recently that substantial advances in chemistry-climate models have made theoretical investigations of the influence of EPPs on long term changes in atmospheric chemistry, ozone and climate (e.g., Baumgaertner et al. 2011; Semeniuk et al. 2011; Rozanov et al. 2012). The inclusion of solar protons and auroral electrons are also now recommended now for the study of long term evolution of stratospheric ozone and climate (Eyring et al. 2013). In this subsection we describe the processes responsible for the ozone layer response to different EPPs types, such as solar protons, galactic cosmic rays (GCR), auroral and relativistic electron precipitations and illustrate them using observational data and model results from available publications. Special attention is paid to the parameterization of these processes for the atmospheric models.

\subsubsection{The processes involved}

All precipitating energetic particles ionize neutral molecules (e.g., $\mathrm{N}_{2}$ and $\mathrm{O}_{2}$ ) in the Earth's atmosphere and produce chemically active radicals as N, NO, H and $\mathrm{OH}$ which can be further transformed by gas phase chemistry. The distribution of the ionization rates in space and time depends on the particle type, due to interactions with the variable heliomagnetic and geomagnetic fields. Despite this, the direct chemical effects are considered to be more or less the same consisting of additional production of reactive hydrogen $\left(\mathrm{HO}_{x}=\mathrm{H}+\mathrm{OH}+\mathrm{HO}_{2}\right)$ and nitrogen $\left(\mathrm{NO}_{x}=\mathrm{N}+\mathrm{NO}+\mathrm{NO}_{2}\right)$ oxides which are able to accelerate catalytic ozone destruction cycles. The subsequent fate of the reactive nitrogen and hydrogen species produced by particles depends on their lifetime. The response of the short-lived $\mathrm{HO}_{x}$ is highly localized in time and space and can be observed only during the particle precipitation event in the region where the ionization occurs. $\mathrm{NO}_{x}$ is more stable and can be transported by atmospheric winds. Therefore, $\mathrm{NO}_{x}$ enhancement can be observed after the event and far from the production area influencing the ozone balance with implications for the ozone layer. This chain of processes was first discussed by Nicolet (1975), investigated using a global 2-D model by Solomon et al. (1982) and was called an indirect EPP effect by Randall et al. 
(2007). In the following subsections we describe the direct and indirect effects of EPP in the stratosphere, mesosphere and lower thermosphere as well as their representation in models and observation data.

\section{Direct chemical effects}

In the lower thermosphere and upper mesosphere the heavy ion clusters do not play an important role and the humidity is small. Therefore, the production of reactive hydrogen can be neglected and the reactive nitrogen production can be mostly described by the following reactions (Nicolet 1975; Porter et al. 1976; Rusch et al. 1981; Sinnhuber et al. 2012), see also the equations $7-15$.

$$
\begin{array}{r}
N_{2}+e^{*}=>N^{+}+N\left({ }^{4} S\right)+2 e^{-}, \\
N_{2}+e^{*}=>N^{*}+N\left({ }^{4} S\right)+e^{-}, \\
N_{2}+e^{*}=>N_{2}^{+}+2 e^{-}, \\
N_{2}^{+}+O=>N O^{+}+N\left({ }^{4} S\right), \\
N_{2}+N^{+}=>N_{2}^{+}+N\left({ }^{4} S\right), \\
N_{2}^{+}+e^{-}=>N^{*}+N\left({ }^{4} S\right), \\
N O^{+}+e^{-}=>N\left({ }^{4} S\right)+O, \\
N^{+}+O=>O^{+}+N\left({ }^{4} S\right), \\
N^{*}+O_{2}=>N O+O .
\end{array}
$$

The produced reactive nitrogen can be also destroyed via the so-called "cannibalistic" reaction $\mathrm{N}\left({ }^{4} \mathrm{~S}\right)+\mathrm{NO}=>\mathrm{N}_{2}+\mathrm{O}$. In the presence of the solar light the photolysis of $\mathrm{NO}\left(\mathrm{NO}+\mathrm{h} \nu=>\mathrm{N}\left({ }^{4} \mathrm{~S}\right)+\mathrm{O}\right)$ followed by "cannibalistic" reaction also leads to removal of reactive nitrogen species from the atmosphere. In order to represent the reactive nitrogen source in the lower thermosphere the simplified subset of the reactions involving five main ions is usually applied (Marsh et al. 2007), which however is a good approximation only at altitudes above $100 \mathrm{~km}$, see Nieder et al. (2014). For the mesosphere and stratosphere all relevant reactions should be included in the model (Egorova et al. 2011). The treatment of this process in the atmosphere below $60 \mathrm{~km}$ can be also parameterized using careful consideration of the chemical balances. Porter et al. (1976) suggested that $1.27 \mathrm{~N}$ atoms are produced per ion pair, of which $55 \%$ are in exited $\left(\mathrm{N}^{*}\right)$ and $45 \%$ are in ground $\mathrm{N}\left({ }^{4} \mathrm{~S}\right)$ states. It should be noted that this parameterization is not accurate in the upper mesosphere/lower thermosphere because of the increasing impact of ion chemistry (e.g., Sinnhuber et al. 2012; Nieder et al. 2014). It is also not clear how the parameterized production rate would be changed due to neutral chemistry which tends to diminish the $\mathrm{NO}_{x}$ production via "cannibalistic" reaction.

As it was shown in Section 3.1, below $80 \mathrm{~km}$ the chemistry of the charged components becomes more complicated due to the presence of water vapor, negative ions and heavy ion clusters. The processes responsible for the reactive nitrogen production in this layer are almost the same, but the additional source of active hydrogen cannot be neglected. The main processes responsible for the $\mathrm{HO}_{x}$ formation were considered by Solomon et al. (1981), Aikin (1994) and recently reevaluated by Sinnhuber 
et al. (2012). They pointed out that $\mathrm{O}_{2}{ }^{+}$is able to directly produce hydroxyl radical via $\left(\mathrm{O}_{2}{ }^{+}+\mathrm{H}_{2} \mathrm{O}+\mathrm{H}_{2} \mathrm{O}=>\mathrm{H}+\mathrm{H}_{2} \mathrm{O}+\mathrm{OH}+\mathrm{O}_{2}\right)$ and facilitates further clustering of proton hydrates $\left(\mathrm{H}^{+}\left(\mathrm{H}_{2} \mathrm{O}\right)_{m}\right)$ followed by dissociative recombination producing atomic hydrogen $\left(\mathrm{H}^{+}\left(\mathrm{H}_{2} \mathrm{O}\right)_{m}+\mathrm{e}=>\mathrm{H}+m\left(\mathrm{H}_{2} \mathrm{O}\right)\right)$. Fast reactions of atomic hydrogen with oxygen and ozone almost immediately lead to the production of $\mathrm{OH}$ and $\mathrm{HO}_{2}$ radicals. The latter process does not play an important role below $60-70 \mathrm{~km}$, where the electron concentration is rather small. Besides these processes there are other ion reactions producing $\mathrm{HO}_{x}$ from hydrogen containing species (e.g., $\mathrm{O}_{2}{ }^{+}+\mathrm{e}=>\mathrm{O}\left({ }^{3} \mathrm{P}\right)+\mathrm{O}\left({ }^{1} \mathrm{D}\right)$ followed by $\left.\mathrm{H}_{2} \mathrm{O}+\mathrm{O}\left({ }^{1} \mathrm{D}\right)=>\mathrm{OH}+\mathrm{OH}\right)$, which can also contribute to $\mathrm{HO}_{x}$ production by energetic particles. These processes may produce up to two $\mathrm{HO}_{x}$ molecules per ion pair and this number is used for the parameterization of the ion chemistry proposed by Solomon et al. (1981), which suggested that below $60 \mathrm{~km}$ altitude two $\mathrm{HO}_{x}$ molecules are produced per ion pair formed, while above $80 \mathrm{~km}$ this number drops towards 1.2 $\mathrm{HO}_{x}$ molecules and becomes very small above $85 \mathrm{~km}$. As it was mentioned before the life time of $\mathrm{HO}_{x}$ is relatively short because it can be transformed back to $\mathrm{H}_{2} \mathrm{O}$ (e.g., $\left.\mathrm{OH}+\mathrm{HO}_{2}=>\mathrm{H}_{2} \mathrm{O}+\mathrm{O}_{2}\right)$. The recombination of heavy clusters can also complicate the parameterization leading to the production of the nitric acid via the equations 16- 17 .

$$
\begin{gathered}
H^{+}\left(\mathrm{H}_{2} \mathrm{O}\right)_{n}+\mathrm{NO}_{3}^{-}\left(\mathrm{HNO}_{3}\right)_{m}=>(m+1)\left(\mathrm{HNO}_{3}\right)+n\left(\mathrm{H}_{2} \mathrm{O}\right) \\
\mathrm{N}_{2} \mathrm{O}_{5}+\mathrm{H}^{+}\left(\mathrm{H}_{2} \mathrm{O}\right)_{n}=>\mathrm{HNO}_{3}+\mathrm{HNO}_{3}+(n-1)\left(\mathrm{H}_{2} \mathrm{O}\right) .
\end{gathered}
$$

Aikin (1997) and Verronen et al. (2008) pointed out that the first reaction plays a major role above $40 \mathrm{~km}$, while the second reaction can substantially contribute only around $30 \mathrm{~km}$. The importance of these reactions was emphasized by Verronen et al. (2011b), which showed that a proper treatment of $\mathrm{HNO}_{3}$ production is necessary to obtain realistic simulation of $\mathrm{HO}_{x}$ production and ozone response to EPP. Because the $\mathrm{HNO}_{3}$ lifetime during polar winter is rather long the application of the abovementioned parameterization of $\mathrm{HO}_{x}$ production by Solomon et al. (1981) could lead to faster removal of additional $\mathrm{HO}_{x}$ from the atmosphere and some underestimation of the ozone depletion.

The treatment of the reactive nitrogen and hydrogen production by EPP can be done using different approaches. The most straightforward way is to include a complete description of the neutral and ion chemistry in the model. Despite some uncertainties in the kinetic reaction coefficients and products of ion recombination reactions this approach is the most solid and avoids any mentioned earlier problems related to the separation of neutral and ion chemistry. On the other hand this approach is very computationally expensive. The treatment of ion chemistry requires hundreds of additional reactions and more than fifty species substantially reducing model performance and limiting its application for climate studies. The extensive representation of the ion chemistry has only been implemented in box models UBAIM (Kazil et al. 2003) and UBIC (Winkler et al. 2009) as well as in 1-D SIC (Verronen et al. 2002) and MGO (Ozolin et al. 2009) models which cannot be used for proper climate and ozone layer studies and in 3-D CCM SOCOL (Egorova et al. 2011). However, the latter model was exploited only for short term (up to 1 month) experiments due to very large consumption of computer time. The other method of EPP treatment is to use the parameterizations of the reactive nitrogen production based on Porter et al. (1976) with an extension into the upper mesosphere and lower thermosphere provided only recently by Sinnhuber et al. (2012) and Nieder et al. (2014), and parameterizations of the $\mathrm{HO}_{x}$ production rate based on Solomon et al. (1981) recommendations. The 
$\mathrm{HNO}_{3}$ production due to ion recombination can be parameterized as suggested by Verronen and Lehmann (2013). The implementation of these parameterizations is rather easy and does not slow down model computations. The study of atmospheric effects of the October 2003 SEP with a 3-D model using extensive description of ion chemistry and parameterizations by Porter et al. (1976) and Solomon et al. (1981) did not show dramatically different results (Egorova et al. 2011) which confirms that the above described parameterizations or their modifications can be a reasonable way to include ionization by solar protons to chemistry-climate models.

There are also a few observations available of a chemical impact of magnetospheric electron precipitation into the middle atmosphere: an increase in $\mathrm{OH}$ for a number of energetic electron precipiation events above $70 \mathrm{~km}$ (Verronen et al. 2011a; Andersson et al. 2012) clearly mapping into geomagnetic latitudes connecting to the radiation belts, and showing longitudinal inhomogeneities there (Andersson et al. 2014a). The observation of a weak but significant increase of $\mathrm{NO}_{2}$ related to geomagnetic activity in the upper stratosphere at $45-52 \mathrm{~km}$ altitude in Northern latitudes connecting to the radiation belts (Friederich et al. 2014).

\section{Indirect effects}

The sequence of the indirect processes responsible for the influence of EPPs on ozone consists of the chemical transformation of reactive nitrogen and hydrogen followed by the transport of the relatively long-lived species out of the production area. As was mentioned earlier the reactive nitrogen and hydrogen oxides formed by the EPP induced ionization can be deactivated through the neutral chemistry. Both $\mathrm{NO}_{x}$ and $\mathrm{HO}_{x}$ can be partially deactivated in the lower stratosphere by $\mathrm{OH}$ and $\mathrm{NO}_{2}$ recombination $\left(\mathrm{OH}+\mathrm{NO}_{2}+\mathrm{M}=>\mathrm{HNO}_{3}+\mathrm{M}\right)$. In the troposphere the removal of EPP products occurs via the wet and dry deposition of hydrogen and nitrogen containing species. The absence of the wet and dry deposition treatment in a model could lead to accumulation of the EPP products in the troposphere.

For SEP and in particular for the magnetospheric electrons the ionization takes place well above the maximum of the ozone distribution. In this case the downward transport of relatively long-lived $\mathrm{NO}_{x}$ plays a major role. This process is the most effective inside the polar vortex area where the downward air motion prevails and during the polar night when the deactivation of $\mathrm{NO}_{x}$ by solar irradiance does not operate. The downward transport of the $\mathrm{NO}_{x}$ produced by SEP and relativistic electrons in the mesosphere and upper stratosphere can be qualitatively well represented in most of the available models. The simulation of production and downward propagation of $\mathrm{NO}_{x}$ produced by low energy auroral electrons in the thermosphere does not constitute a technical problem for the models extending to the upper atmosphere and including ion chemistry, however the quantitative representation of the downward propagation is far from perfect. Additional parameterizations of the production and transport are necessary for some of the existing low-top models because the vertical domain does not cover the thermosphere.

The response of the ozone to the $\mathrm{NO}_{x}$ and $\mathrm{HO}_{x}$ enhancement caused by EPP depends on the altitude. Above the tropopause the ozone can be destroyed via the intensification of the well-known catalytical ozone destruction cycles involving $\mathrm{HO}_{x}$ and $\mathrm{NO}_{x}$ (Brasseur and Solomon 2005; Lary 1997). The ozone depletion through the $\mathrm{HO}_{x}$ related cycles should be collocated with the ionization rates in space and time, 
while the ozone response to $\mathrm{NO}_{x}$ enhancement can be observed in the remote areas and with some time delay caused by the propagation of $\mathrm{NO}_{x}$ out of the production area. In the troposphere the magnitude and even the sign of the ozone response depends on the background concentration of the ozone precursors. In the relatively clean environment with low level of $\mathrm{NO}_{x}$ concentration the ozone production can be enhanced by additional $\mathrm{NO}_{x}$ due to photosmog reactions (Wang et al. 1998). The estimation of this effect requires proper treatment of tropospheric chemistry including the removal of the nitrogen and hydrogen containing species by wet and dry deposition.

The indirect effects of energetic particle precipitation and the downward transport of $\mathrm{NO}_{x}$ from the low thermosphere, based on data from several satellite instruments, has been carefully demonstrated by Randall et al. (2007) using HALOE and POAM observations and by Funke et al. (2014) using the complete MIPAS data set.

The variation of $\mathrm{NO}_{x}$ flux formed by energetic particle precipitation over 1992-2005 was $0.1-2.6 \mathrm{Gmol} / \mathrm{yr}$ (Randall et al. 2007). The maximum values were obtained in 1994 and 2003. The interannual variability correlates well with several energetic particle precipitation indices, including auroral hemispheric power and the hemispheric power of moderate-energy electrons; the former - an energy flux in the lower thermosphere - was estimated by electron fluxes of energy from $50 \mathrm{eV}$ to $20 \mathrm{keV}$ and the latter electron precipitation to the upper mesosphere - based on measurements of electrons with energies between $35 \mathrm{keV}$ and $2.5 \mathrm{MeV}$. These results are important in exploring the interaction between the upper and lower atmosphere and assessing the effect of energetic particle precipitation on ozone.

A wide variety of atmospheric models can be successfully used to study different processes, however, only chemistry-climate models (CCM) have a potential to mimic the entire chain of processes involved in the propagation of the signal from the ionization by EPP to the resulting ozone response. The state-of-the-art CCMs (Morgenstern et al. 2010) include detailed treatment of the neutral chemistry and photolysis as well as transport of chemical species. The ability of CCM to simulate the response of the net heating rates to the ozone changes (Forster et al. 2011) and translate the net heating rate perturbation to the changes of general circulation and surface climate open a way to study the entire chain of processes connecting EPP and climate variability.

\subsubsection{Modeling and observations of ozone changes caused by EPP}

\section{Auroral electrons}

Low energy (1-30 KeV) auroral electrons precipitate from the magnetospheric plasmasheet (Brasseur and Solomon 2005). Their penetration depth is rather small and the energy deposition occurs mainly in the lower thermosphere (above $90 \mathrm{~km}$ ) and confines to the auroral ovals located over $60-75^{\circ}$ geomagnetic latitude band (Baker 2000; Barth et al. 2003). The overall intensity of the auroral electron precipitation depends on the geomagnetic activity level maximizing during the declining phase of the solar activity cycle (Emery et al. 2008; Marsh et al. 2007). The auroral electrons represent one of the main sources of nitrogen oxides in the thermosphere. This theoretically expected effect was confirmed by the analysis of observations. The high level of NO concentration in the auroral zone has been observed by the SNOE instrument (Baker 2000) and is illustrated in the Fig. 27. 


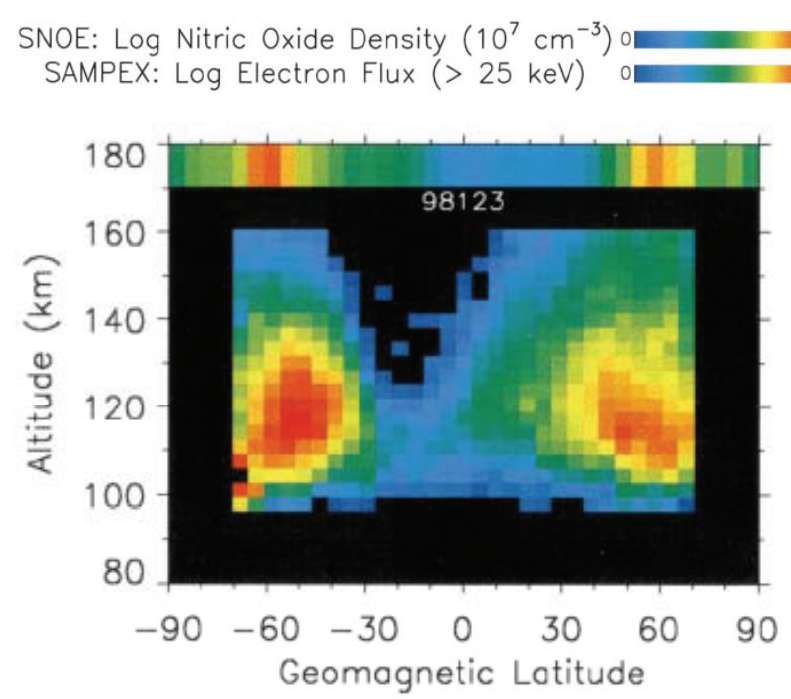

Fig. 27 A comparison of SNOE nitric oxide measurements and SAMPEX electron $(\mathrm{E}>25$ $\mathrm{keV}$ ) measurements for days 123 of 1998 (3 May). SNOE data are plotted in a color scale ranging $\sim 1 \times 10^{7} \mathrm{~cm}^{-3}$ (blue) to $\sim 3 \times 10^{7} \mathrm{~cm}^{-3}$ (red), while the SAMPEX electrons range over about a factor of 1000 in intensity for the same color range. The nitric oxide density in the auroral region is larger than the density in the equatorial region. The figure is adopted from Baker (2000), with permission from Elsevier.

The figure shows that the measured NO concentration maximizes in the lower thermosphere slightly below $110 \mathrm{~km}$ and in the location of the auroral ovals. During the polar night thermospheric $\mathrm{NO}_{x}$ can penetrate downward providing an important source of nitrogen oxides for the mesosphere and stratosphere. The downward propagation of the thermospheric $\mathrm{NO}_{x}$ continuously occurs during the polar night due to the complicated interaction between diffusive and advective transport (Smith et al. 2011), but it is most pronounced during the recovery after major stratospheric warming events (Funke et al. 2005) or during substantial geomagnetic perturbations caused by explosive solar events. As it was mentioned before, the downward propagation of $\mathrm{NO}_{y}$ has been carefully demonstrated also by satellite observations (Randall et al. 2007; Funke et al. 2014). Strong increase of $\mathrm{NO}_{x}$ and subsequent ozone depletion were analyzed by Seppälä et al. (2007) using the observation of $\mathrm{NO}_{2}$ and ozone by GOMOS and POAM-III satellite instruments. The Figure 28 from Seppälä et al. (2007) illustrates the ozone depletion caused by SEP event in November 2003 and by auroral electrons in March 2004. Contrasting one year a large indirect effect in $N O_{y}$ suggests that the low ozone values in spring of this year compared to the multi-annual mean are due to the indirect effect, but does not really prove it. It is necessary to analyse an ensemble of many years with varying $N O_{y}$ indirect effect to make a statement of the statistical significance of this effect on ozone. Therefore, the observational evidences for the ozone loss caused by auroral electrons are still very limited.

The $\mathrm{NO}_{x}$ production by auroral electrons was included by Marsh et al. (2007) in high-top CCM WACCM using the ionization rates prescribed as a function of geomagnetic $\mathrm{K}_{p}$ index. For the low-top $\left(Z_{t o p}<90 \mathrm{~km}\right)$ models this approach cannot be used due to the absence of the thermosphere. Therefore, alternative parameterization of the 
Ozone at $40 \mathrm{~km}$

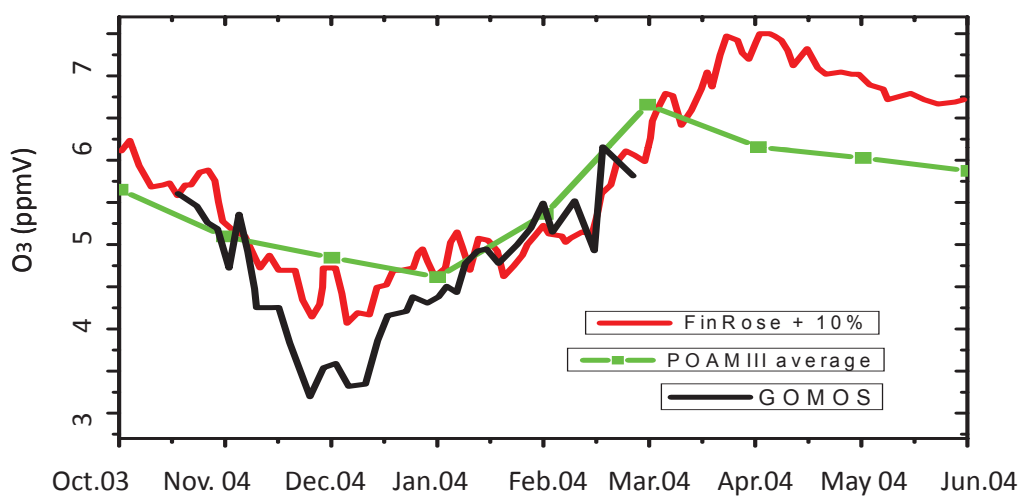

Fig. 28 Polar $\mathrm{O}_{3}$ mixing ratios in the Northern Hemisphere. The average long-term $\mathrm{O}_{3}$ mixing ratio is presented at the $40 \mathrm{~km}$ level from the POAM average (green line) and together with that from the FinROSE model (red line). These are to be contrasted with the $\mathrm{O}_{3}$ mixing ratios measured during October 2003 to June 2004 by the GOMOS (black line, nighttime) The figure is modified after Seppälä et al. (2007).

nitrogen oxides source due to auroral electron precipitation based on geomagnetic $\mathrm{A}_{p}$ index was developed by Baumgaertner et al. (2009). This parameterization is based on the HALOE data, which have a very sparse sampling in the high latitude winter, therefore the magnitude of the indirect effect could be quite considerably underestimated. Using CCM EMAC with the parameterized influx of nitrogen oxides from the thermosphere (Baumgaertner et al. 2011) studied the atmospheric response to the level of geomagnetic activity and found significant ozone depletion in the polar winter stratosphere $(\sim 15-50 \mathrm{~km})$. The ozone depletion due to auroral electrons simulated by Baumgaertner et al. (2011) is illustrated in Figure 29.

The magnitude of the simulated stratospheric ozone depletion is around 0.5-1 ppmv (or 10-20\%) in the middle and upper stratosphere mostly over the polar area. Baumgaertner et al. (2011) also found total column ozone loss of up to 35 DU. Also Reddmann et al. (2010) used an upper boundary condition based on MIPAS observations to derive the ozone loss due to the indirect effect. Similar parameterization of the $\mathrm{NO}_{x}$ production by auroral electrons was applied by Rozanov et al. (2012) to simulate the effects of different particles on the chemistry and climate. They found that the annual mean ozone depletion is the most pronounced in the mesosphere (more than 10\%) but it is also visible in the middle stratosphere reaching $3-4 \%$. The potential importance of solar activity for future climate was recently studied by Rozanov et al. (2012) using the CCM SOCOL. They assume $60 \%$ drop of $\mathrm{A}_{p}$ in the future due to a potential decline of the solar activity discussed by Abreu et al. (2008); Barnard et al. (2011). In addition to the combined effects of the solar activity decrease presented by Rozanov et al. (2012) we show here the response of $\mathrm{NO}_{y}$ and ozone to the hypothetical decrease of geomagnetic activity in the future. The simulated annual mean and zonal mean changes of $\mathrm{NO}_{y}$ are shown in Figure 30.

The annual decrease of the $\mathrm{NO}_{y}$ caused by the future decline of solar activity is evident and statistically significant in the entire stratosphere. The decrease of $\mathrm{NO}_{y}$ maximizes in the high-latitude mesosphere reaching almost $80 \%$. In the middle strato- 


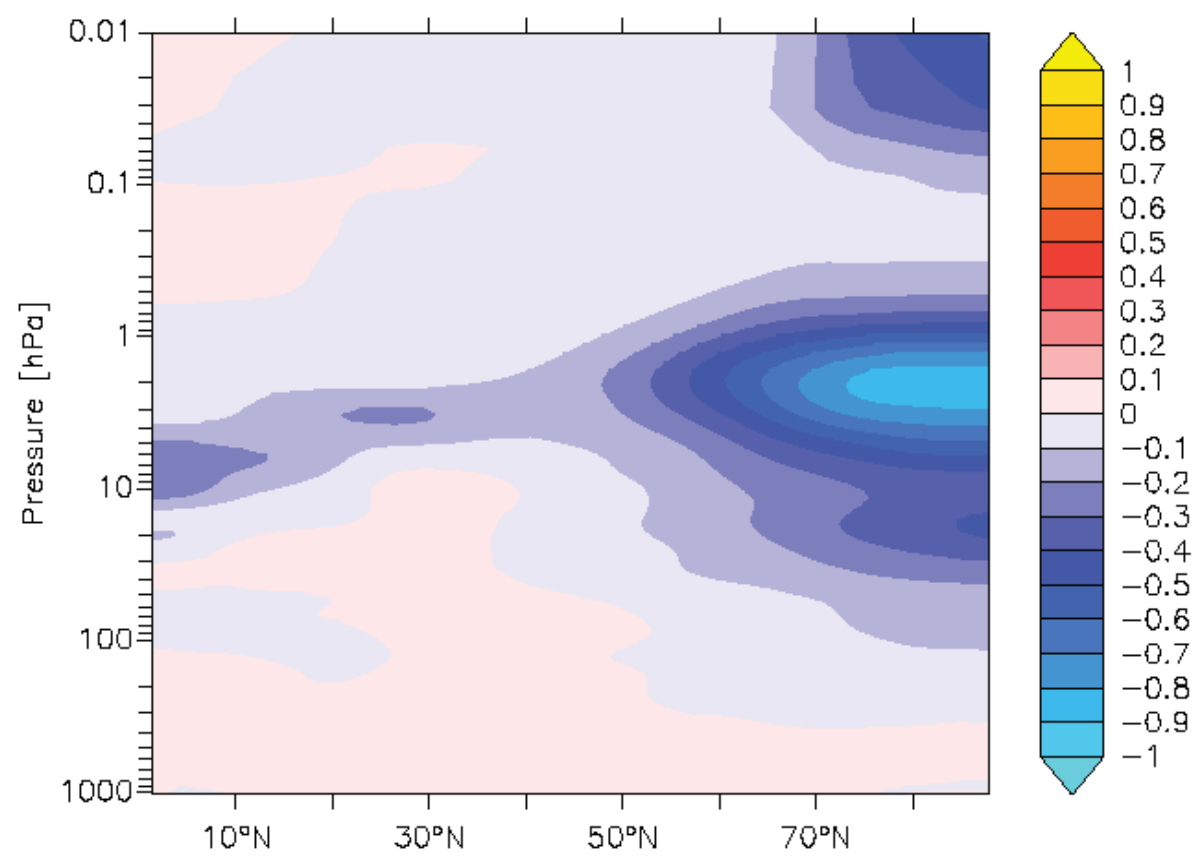

Fig. 29 Ozone mixing ratio difference (ppmv) between two 9-year long CCM EMAC model runs with strong $\left(\mathrm{A}_{p}=25\right)$ and weak $\left(\mathrm{A}_{p}=0\right)$ geomagnetic activity level. The results are averaged over boreal winter months. Red-yellow/blue colours indicate positive/ negative differences. The figure is from Baumgaertner et al. (2011).

sphere the decrease of $\mathrm{NO}_{y}$ is around 10-20\%. In agreement with the $\mathrm{NO}_{y}$ the model simulates ozone increase by up to $20 \%$ in the polar mesosphere and up to $2 \%$ in the middle stratosphere. The presented results suggest that the auroral electrons are important for the stratospheric ozone balance. The magnitude of the obtained ozone response in the high latitude stratosphere is comparable or even exceeds the ozone response to the solar EUV irradiance changes (Austin et al. 2008). It should be noted, however, that future solar activity can not be predicted therefore this can be considered only as a possible "low active Sun" scenario (Usoskin 2013).

\section{Radiation belt electrons}

High-energy electrons, from about $30 \mathrm{keV}$ up to several $\mathrm{MeV}$, are produced from the outer radiation belts, see (Bazilevskaya et al. 2008) and Section 2.2. Their precipitation to the atmosphere (Rozanov et al. 2012) is caused by complex wave-particle interactions and mostly confined to subauroral latitudes (Millan and Thorne 2007) with a penetration depth from $90 \mathrm{~km}$ for $30 \mathrm{keV}$ electrons and down to $50 \mathrm{~km}$ for $2 \mathrm{MeV}$ electrons. Meredith et al. (2011) mentioned that fluxes of radiation belt electrons can be enhanced during geomagnetic perturbations. The possibility of mid-stratospheric interannual ozone variations connected to radiation belt electrons is discussed in Sinnhuber et al. (2006). Sinnhuber et al. (2012) in their last recent review paper summarized 

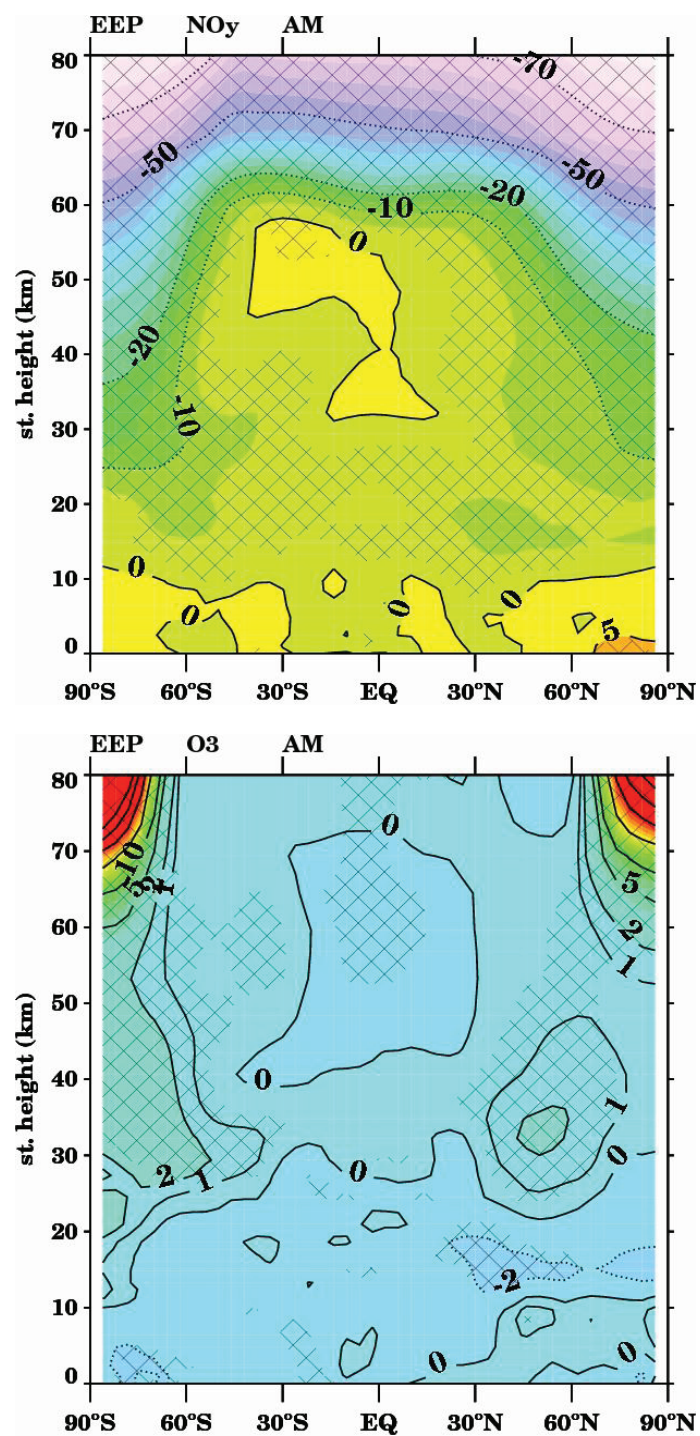

Fig. 30 The simulated annual and zonal mean difference (\%) of $\mathrm{NO}_{y}$ (upper panel) and ozone (lower panel) caused by $60 \%$ drop of $\mathrm{A}_{p}$ index. Hatching represent the areas where the statistical significance exceed $90 \%$ level.

knowledge on atmospheric chemistry effects of variations of EPP as well as relativistic electrons, from the radiation belt. Sinnhuber et al. (2012) noticed that there can be an impact of relativistic electron precipitation on ozone in the mid-stratosphere, however the coupling mechanism is still not clear.

Recent research (Andersson et al. 2014b) has shown that energetic electron precipitation (EEP), as mapped by the MEPED detector onboard NOAA/POES satellites during 2002-2012, caused up to $90 \%$ (short-time scale) destructions of mesospheric 
ozone at $60-80 \mathrm{~km}$ altitudes and up to $34 \%$ (on solar cycle time scale) destructions of mesospheric ozone at $70-80 \mathrm{~km}$, as seen by 3 satellite instruments GOMOS, MLS and SABER. Most studies so far on atmospheric effects of energetic particle precipitation concentrated on the indirect particle precipitation effect caused by the production of odd nitrogen $\left(\mathrm{NO}_{x}\right)$ in the polar upper atmosphere, its subsequent transport to lower altitudes inside the wintertime polar vortex, depletion of ozone in the stratosphere, and effects on the radiative balance of the middle atmosphere, which may further couple to atmospheric dynamics and propagate downwards by changing polar winds and atmospheric wave propagation through wave-mean flow interaction.

However in spite of some observation results (Andersson et al. 2014b) the current knowledge related to electron precipitations and its impact on the atmosphere is still an open issue, and should be discussed here.

The effects of precipitating relativistic electron fluxes have been analyzed by Callis et al. $(1991,1996,1998,2002)$, based however mainly on model studies. The model used in these papers model is driven by ionization rates which are quite uncertain in the mesosphere and stratosphere.

The results presented in the paper (Callis et al. 1991) were based on the assumption that $1 / 3$ (which may be an overestimation) of the electron fluxes reach the middle atmosphere and disturb ozone in the stratosphere and mesosphere via $\mathrm{NO}_{x}$ production (using model simulations). Because of the lack of measurements at lower altitudes during geomagnetic storms, the variability of this assumption is unclear. Such possible effects were studied by Pesnell et al. $(1999,2000)$ on the basis of observations from onboard the UARS spacecraft. Details may be found also in the reviews of Krivolutsky and Repnev (2012); Repnev and Krivolutsky (2010).

Atmospheric ionization and NO formation rates at auroral and sub-auroral latitudes from 13th June 1979 to 4th June 1988 were calculated using the data on captured electron fluxes with energies from $30 \mathrm{keV}$ to $15 \mathrm{MeV}$ (Callis et al. 1991). Maxima in the long-term $\mathrm{NO}_{y}$ variation were measured in late 1984 and early 1985 (Fig. 31). Based on 2-D modelling of $\mathrm{NO}_{y}$ with the contribution of relativistic electron precipitation allowed for, it was inferred that:

1. Relativistic electron precipitation (REP) from the magnetosphere to the middle atmosphere is a significant time-variable source of odd nitrogen accounting for about a $30-35 \%$ contribution due to $\mathrm{N}_{2} \mathrm{O}$ for 1979-1985.

2. Subsequent downward transport results in the global enhancement of stratospheric odd nitrogen with a maximum in late 1984-early 1985 reaching a globally integrated value of $35-40 \%$ of global value.

3. After 1985, the global $\mathrm{NO}_{y}$ diminishes with the decreasing REP contribution.

4. Odd nitrogen enhancement in the mid- and high-latitude lower stratosphere $\left(30^{\circ}\right.$ - $60^{\circ}$ ) in both hemispheres could have significantly contributed to the large (up to $10 \%$ ) ozone loss in an altitude range of $14-26 \mathrm{~km}$ at high latitudes of the Southern Hemisphere and somewhat smaller high-altitude loss at high latitudes of the Northern Hemisphere.

5. The results obtained suggest a clear and close connection between long-term solar variations, the magnetosphere state, and the chemical state of the middle and lower atmosphere (Callis et al. 1991). Callis et al. (1996) analyzed satellite measurements of relativistic electron precipitation (REP) events in the Northern and Southern Hemispheres). The calculated ionization and NO formation rates were compared with direct satellite measurements of NO. The NO increase at $51-120 \mathrm{~km}$ altitude by $(2.4-$ $3.5) \cdot 10^{14}$ molecule $/ \mathrm{cm}^{2}$, which is $2.5-3.4 \% \mathrm{NO}_{y}$ in a column between 22 and $120 \mathrm{~km}$, 

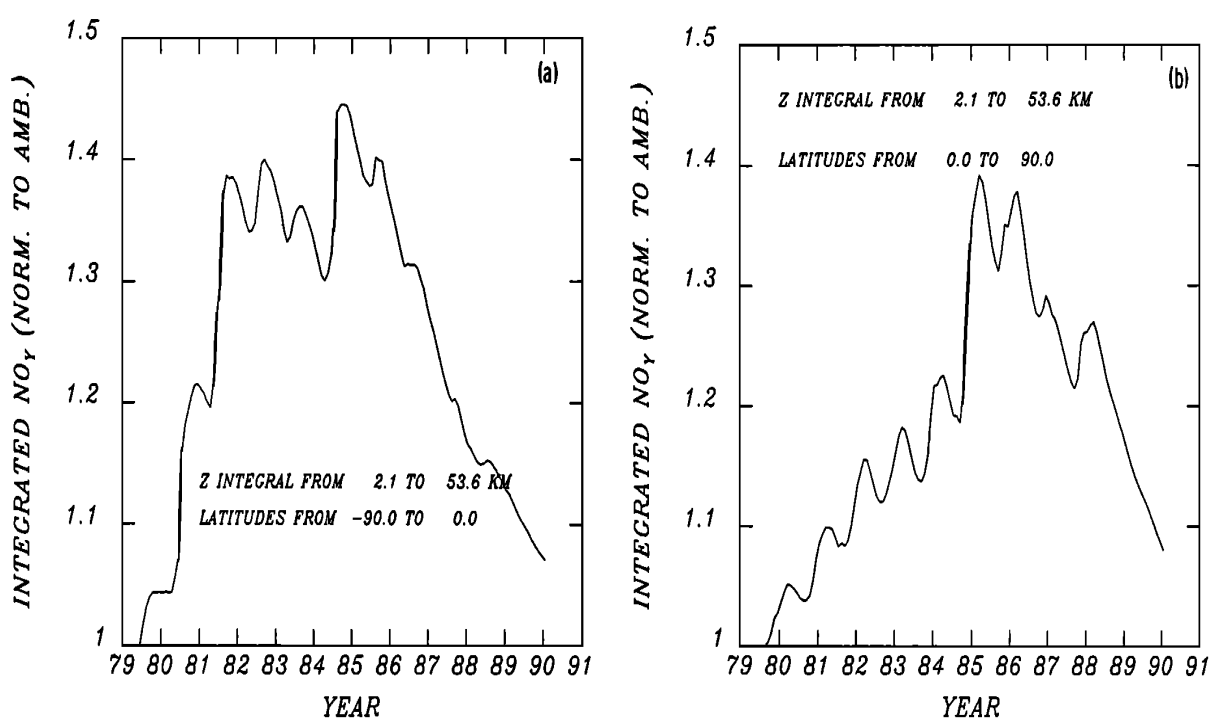

Fig. 31 Calculated hemispheric integral odd nitrogen changes normalized to values in the absence of a relativistic electron precipitation source during the period from 1979 to January 1990. (a) Southern Hemisphere: integral between 2.1 and $53.6 \mathrm{~km}, 0-90^{\circ} \mathrm{S}$; (b) Northern Hemisphere: integral between 2.1 and $53.6 \mathrm{~km}, 0-90^{\circ} \mathrm{N}$. Reprinted from Callis et al. (1991), with permission from Wiley.

is assumed to have resulted from the NO enhancement above $80-85 \mathrm{~km}$ caused by precipitating electrons of energy $\mathrm{E} \leq 1.0 \mathrm{MeV}$. Callis et al. (2002) presented a comparison between the daily HALOE mesospheric ozone values and the ones calculated with a 2-D chemistry and transport model for 1992-1997. The results agreed very well when the model allowed for the effect of energetic electron precipitation (EEP) from the outer electron capture magnetosphere region. The mesosphere largely contributed $(16 \%)$ to stratospheric $\mathrm{NO}_{y}$ above $25 \mathrm{~km}$ at latitudes between $70^{\circ} \mathrm{S}$ and $70^{\circ} \mathrm{N}$, which was basically ( $74 \%$ ) due to precipitating energetic electrons. The rest of the mesospheric contribution $(26 \%)$ was associated with the effect of auroral electron precipitation and solar EUV and solar X-rays on NO formation (via the model upper boundary condition).

In spite of the material presented above, we need to remind that the impact of radiation belt relativistic electrons on the stratosphere and mesosphere is unresolved. However the observational evidence so far suggests that relativistic electrons have an impact on the $\mathrm{NO}_{x}$ budget of the middle atmosphere below $\sim 70 \mathrm{~km}$ (Sinnhuber et al. 2012; Rozanov et al. 2012). And there is newer observational evidence for the direct impact of relativistic electrons on the $\mathrm{NO}_{x}$ budget of the lower mesosphere and stratosphere (Sinnhuber et al. 2014; Friederich et al. 2014) which show that the impact of EEP below $70 \mathrm{~km}$ must be low even at periods of very high geomagnetic activity (Sinnhuber et al. 2014), though there is a significant, but small impact maximizing around $48 \mathrm{~km}$ (Friederich et al. 2014). There is also clear evidence for an energetic electron precipitating effect above $70 \mathrm{~km}$ altitude from $\mathrm{OH}$ observations as shown in Verronen et al. (2011a); Andersson et al. (2012, 2014a). 
Solar protons

Satellite observations and rocket soundings have produced evidence that energetic particles influence ozone, other chemical species and atmospheric temperature. This section now focuses on selected SEP events, which are covered by these observations. It was found after polar ozone content analysis that ozone, measured by Nimbus-4, was reduced strongly in the stratosphere after the SEP event in August 1972 (Heath et al. 1977; Fabian et al. 1979). Following this, the results of rocket measurements of middle atmosphere parameters, launched from a ship at mid-latitudes of the Southern Hemisphere during the SEP of October 1989 (Zadorozhnyi et al. 1992) showed a strong increase in ion number density above $30 \mathrm{~km}$ and nitric oxide concentration above $40 \mathrm{~km}$. The ozone concentration above $35 \mathrm{~km}$ was reduced (maxima of depletion was around $20-25 \%$ at $50 \mathrm{~km}$ ). A corresponding temperature decrease between 40 and $60 \mathrm{~km}$ was also found in this rocket experiment (about $14 \mathrm{~K}$ near $50 \mathrm{~km}$ ). These measurements are in qualitative agreement with photochemical conceptions of the influence of SEPs listed below. Several other SEP events which are of atmospheric relevance are those of 14th July 2000 and 28th October 2003. The July 2000 SEP event was covered by observations from UARS (HALOE instrument), and the October 2003 event by ENVISAT (MIPAS and other instruments). The SPE of 14th July 2000 was studied quickly after the event (Jackman et al. 2001; Krivolutsky 2001) as it was the first chance to study $\mathrm{NO}_{x}$ response (not ozone only) and compare it with simulations, where it was found that the models overestimate $\mathrm{NO}_{x}$ increase induced by SEPs. The SEP event of 28th October 2003 was accompanied by a wide list of species measured by the MIPAS instrument. The results from MIPAS and its comparison with model simulations was the basis to organize the international HEPPA Project (Funke et al. 2011).

It should be mentioned that all the interpretations in such a comparison are based on the theory of photochemistry in the atmosphere (Crutzen 1974) and on fundamental works, which established the efficiencies of atomic oxygen and nitrogen production (Crutzen et al. 1975; Porter et al. 1976; Heaps 1978) and also $\mathrm{HO}_{x}$ production (Solomon and Crutzen 1981) by relativistic protons impact in the atmosphere.

Jackman et al. (1980) using GOES data and photochemical modelling determined rates of NO formation by solar proton flares, during SEP events, on 12th July 1961 $\left(6 \mathrm{~nJ} / \mathrm{cm}^{2} \mathrm{~s}\right), 25 \mathrm{th}$ February $1969\left(4 \mathrm{~nJ} / \mathrm{cm}^{2} \mathrm{~s}\right)$, and 4 th August $1972\left(70 \mathrm{~nJ} / \mathrm{cm}^{2} \mathrm{~s}\right)$. The period of NO formation by solar proton flares can last from several hours to a couple of days. The moderate event of 12 th July 1961, with a relatively soft spectrum, produced $\mathrm{NO}$ in the mesosphere at a rate of $200 / \mathrm{cm}^{3} \mathrm{~s}$, considerably exceeding the mean rate of $\mathrm{NO}$ formation by $\mathrm{N}_{2} \mathrm{O}$ oxidation in the stratosphere $\left(45 / \mathrm{cm}^{3} \mathrm{~s}\right)$. The event of $25 \mathrm{th}$ February 1969, though less intense, produced a larger effect on the budget of NO formed in the stratosphere, where its lifetime is longer. The SEP event of 4th August 1972 caused NO formation in the stratosphere at a rate of $6 \cdot 10^{4} / \mathrm{cm}^{3} \mathrm{~s}$, which is nearly three orders of magnitude higher than those due to any other events. This SEP event accounted for $85 \%$ of the integral flux of energetic solar protons of a total 20-year solar activity cycle. At $38 \mathrm{~km}$ altitude, the ionization rate was a factor of 30.000 greater than that due to GCRs, releasing about $50 \mathrm{~nJ} / \mathrm{cm}^{3}$ between $30-70 \mathrm{~km}$ (Reagan et al. 1981).

Satellite measurements of minor gaseous constituents at altitudes from 6 to $68 \mathrm{~km}$ with the MIPAS instrument aboard ENVISAT during and after the October-November 2003 SEP events showed perturbed chlorine chemistry (von Clarmann et al. 2005). 
$\mathrm{ClO}$ mixing ratios averaged over latitudes $70-90^{\circ} \mathrm{N}$ increased to $0.4 \mathrm{ppbv}$ at night. The amount of $\mathrm{HOCl}$ increased to over $0.3 \mathrm{ppbv}$ above $32 \mathrm{~km}$, which provides indirect evidence of $\mathrm{HO}_{x}$ increase even at low altitudes, due to the 27-31 October 2003 SEP. The simultaneous increase of $\mathrm{ClO}$ and $\mathrm{HOCl}$ is indicative of $\mathrm{HCl}$ destruction either through reaction with $\mathrm{OH}$ or due to cluster ion chemistry. This remains to be clarified in model experiments. Measurements by HALOE also revealed $\mathrm{HCl}$ decrease during SPE of 14th July 2000 (Winkler et al. 2009). $\mathrm{As} \mathrm{HOCl}$ and $\mathrm{ClO}$ mixing ratios decreased in early November (probably due to the $\mathrm{HO}_{x}$ decrease), large amounts of $\mathrm{ClONO}_{2}$ were measured (a 0.4 ppbv average over the polar cap), indicating $\mathrm{ClO}$ transfer to a chlorine reservoir. In Antarctica, above $\sim 40 \mathrm{~km}, \mathrm{ClO}$ daytime amount reached $0.2 \mathrm{ppbv}$, with the largest nighttime increase $(0.3 \mathrm{ppbv})$ observed at $35 \mathrm{~km}$ altitude. The $\mathrm{HOCl}$ increase in the stratosphere of the Southern Hemisphere was half that in the Northern Hemisphere, while being the largest between $60-70^{\circ} \mathrm{S}$. The October-November 2003 SEP events did affect $\mathrm{HNO}_{3}, \mathrm{~N}_{2} \mathrm{O}_{5}$, and $\mathrm{ClONO}_{2}$ in the polar stratosphere, though odd nitrogen formed by protons was transported downward to $30 \mathrm{~km}$ altitudes, simultaneously undergoing chemical conversion (López-Puertas et al. 2005). MIPAS observed several $\mathrm{HNO}_{3}$ increases in the northern polar stratosphere, see Figure 32. The SEP was immediately followed by a 1-2 ppbv increase accounted for by the gas-phase chemical reaction $\mathrm{NO}_{2}+\mathrm{OH}+\mathrm{M} \longrightarrow \mathrm{HNO}_{3}+\mathrm{M}$ accelerated by $\mathrm{OH}$ due to the action of the SEP. A second large increase (by 1-5 ppbv) started around 10th November 2000 and continued until late December 2000. It is attributed to odd nitrogen, $\mathrm{NO}_{x}$, formed in the mesosphere by the major SEPs in late October - early November and transported downwards during November and December. In the upper stratosphere, $\mathrm{NO}_{x}$ was partially converted to $\mathrm{N}_{2} \mathrm{O}_{5}$, which eventually formed $\mathrm{HNO}_{3}$ via ion-cluster reactions. 1-3 days after the major SEPs, $\mathrm{N}_{2} \mathrm{O}_{5}$ increased by 0.1-0.4 ppbv and descended to $30 \mathrm{~km}$ altitude. The second $\mathrm{N}_{2} \mathrm{O}_{5}$ increase up to $1.2 \mathrm{ppbv}$ at $40 \mathrm{~km}$ occurred $12-13$ days after the major SEPs. $\mathrm{ClONO}_{2}$ started increasing to reach 0.4 ppbv $(40 \%)$ at $32 \mathrm{~km}$ altitude 1-2 days after the major SEPs. $\mathrm{NO}_{x}$ enhancement in the Southern Hemisphere was less pronounced (by not more than $0.2 \mathrm{ppbv}$ at altitudes between 40 and $30 \mathrm{~km}$ ) (López-Puertas et al. 2005).

At least one of the three major mesospheric NO sources is associated with solar activity (downward transport from thermosphere, photochemical NO production via $\mathrm{N}_{2} \mathrm{O}+\mathrm{O}\left({ }^{1} \mathrm{D}\right)$, and production by particle-induced ionization). The intensity of the flux from the thermosphere varies, following changes in ionizing solar radiation. Sporadic events, such as SEP events and relativistic electron precipitation, do not seem to cause significant NO changes over a solar activity cycle. Jackman et al. (1990) in their summary paper gives the annual global odd nitrogen production (due common SEPs) in the mesosphere and stratosphere for the period of 1955-1984. Jackman et al. (1990) and Jackman et al. (2005) confirmed that SEPs cannot substantially change the total amount of $\mathrm{NO}_{y}$, as major SEPs (or GLEs, see Section 2.1.4). $\mathrm{NO}_{y}$ changes due to SEPs are observed at high latitudes over a period from several months up to a year (for proton CME of intensity comparable with the August 1972 major SEP or GLE event), and then $\mathrm{NO}_{y}$ relaxes to an unperturbed state. Jackman et al. (1995) showed that all the NO changes caused by solar protons are much less than the background changes due to $\mathrm{N}_{2} \mathrm{O}$ oxidation. It could be concluded that while globally the $\mathrm{NO}_{x}$ production due to GCRs and $\mathrm{N}_{2} \mathrm{O}$ oxidation prevails over the solar events, at high latitudes and for time periods of several months, solar proton events can be the dominating $\mathrm{NO}_{x}$ source. Figure 33 shows a comparison between the August 1972 and October 1989 solar proton events, revealing a remarkable difference in atmospheric ionization. During the SEP 


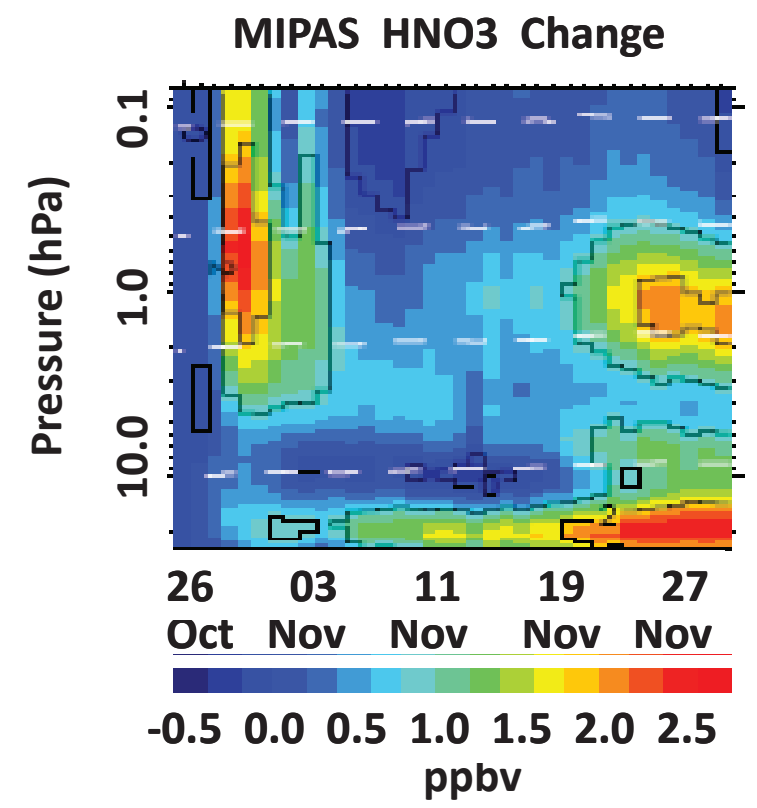

Fig. 32 The observed MIPAS temporal evolutions of the $\mathrm{HNO}_{3}$ enhancements with respect to 26th October averaged over $70 \mathrm{~N}-90 \mathrm{~N}$ (Funke et al. 2011).

event of August 1972, the maximum particle production rate was about $1.2-1.3 \cdot 10^{9}$ ion $/ \mathrm{cm}^{3}$ at altitudes around 45 and $70 \mathrm{~km}$. In October 1989, ionization maximum of about $1.8 \cdot 11^{9}$ ion $/ \mathrm{cm}^{3}$ occurred near $30 \mathrm{~km}$ altitude. $\mathrm{NO}_{x}$ formed by the proton flares continued to affect polar ozone over about one and a half years.

After the October 1989 SEP the SAGE II satellite instrument recorded a substantial increase in $\mathrm{NO}_{2}$ in March 1990, about half of which was thought to be due to the SEP itself. The other half was probably due to the inter-annual differences between the 1990 and 1987 dynamic processes (Jackman et al. 1995). The different effects of the August 1972 and October 1989 SEPs not only resulted from variations in intensity and season, but also from the different atmospheric conditions, and specifically the middle atmosphere composition, under which they occurred. Thus, the August 1972 stratosphere chlorine level was $1.2 \mathrm{ppbv}$, while in 1989, it was already 3 ppbv. The interaction between ozone-depleting nitrogen and chlorine cycles caused the October 1989 SEP effect to be less than it could be with less chlorine present. The effect of the 1972 SEP was more pronounced in the winter Southern Hemisphere due to less solar light causing NO dissociation and to predominantly descending atmospheric motions. The 1989 SEP was more effective in the Northern Hemisphere autumn due to decreasing solar irradiance and descending atmospheric motions beginning to prevail.

Observations made by the UARS HALOE instrument provided estimates of the ozone and $\mathrm{NO}_{x}$ response during the SEP events of 14th July 2000. Figures $34-36$ compare the measurements with model calculations (Krivolutsky et al. 2008). The model response presented at Fig. 34 was obtained by simulations with a $1 \mathrm{D}$ photochemical model (Krivolutsky et al. 2005). This model describes interactions between 50 chemical species in 150 photochemical reactions in the range 0-90 km. The tech- 


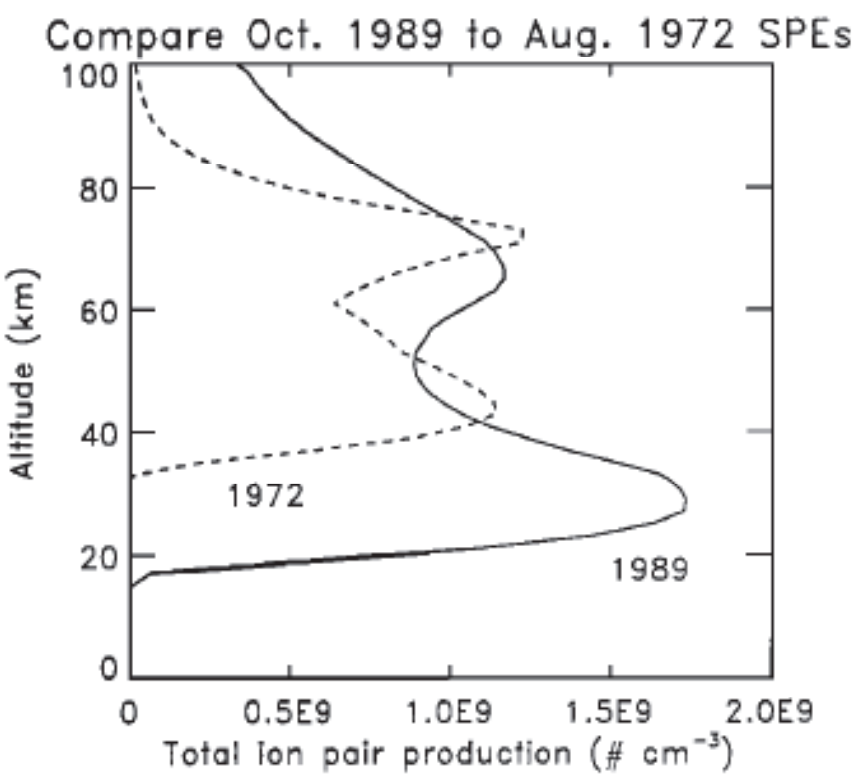

Fig. 33 Estimated ion pair totals (ion pair $/ \mathrm{cm}^{3}$ ) for the August 1972 and October 1989 SEPs. Reprinted from Jackman et al. (1995), with permission from Wiley.

nique of "chemical families" was used in the model to solve the aeronomical part of the basic equations. Turbulent transport by eddy diffusion was included at each step of integration. Current photolysis rates have been calculated for each hour of integration to describe daily variations of photolysis rates. Model simulations with the middle atmosphere GCM (General Climate Model) (Krivolutsky et al. 2006) yielded the dynamical response of temperature and zonal wind caused by SEP-induced ozone depletion. The magnitude of the zonal wind was reduced at all altitudes including lower thermosphere levels. SEP-induced cooling caused by ozone depletion was found in the middle polar atmosphere, but the temperature increased in the lower thermosphere. Such unexpected results are the manifestation of the interaction between gravity waves and the mean motions as initiated by changes of zonal wind at lower altitudes. Solar protons play an important role during SEPs in the sense of photochemistry of ozone and regime of dynamics in polar regions.

The dependence of $\mathrm{NO}_{y}$ on the season (due to changes in EUV-radiation and circulation) and the solar cycle (due to changes in EUV- and X-ray solar radiation and in auroral sources), as well as an asymmetry of polar zones in both hemispheres were revealed. A larger $\mathrm{NO}_{y}$ increase was obtained for the Southern Hemisphere $\left(50^{\circ} \mathrm{S}\right)$. An increase of $3 \%$, depending on the season and year, in the northern polar stratosphere and $8 \%$ in the southern polar stratosphere (at latitudes between $50^{\circ}$ and $90^{\circ}$ and altitudes from the tropopause to $50 \mathrm{~km}$ ) was obtained. The amount of $\mathrm{NO}_{y}$ at the South Pole in the middle of July was found to increase by $10 \%$ to altitudes as low as 30 $\mathrm{km}$, whilst at the North Pole in winter, $\mathrm{NO}_{y}$ increased only above $40 \mathrm{~km}$ altitude. The global budget of stratospheric $\mathrm{NO}_{y}$ was enhanced by thermospheric sources by 1 to 2.5\%. The August 1972 and October 1989 SEPs contributed 15\% and 11\%, respectively, 

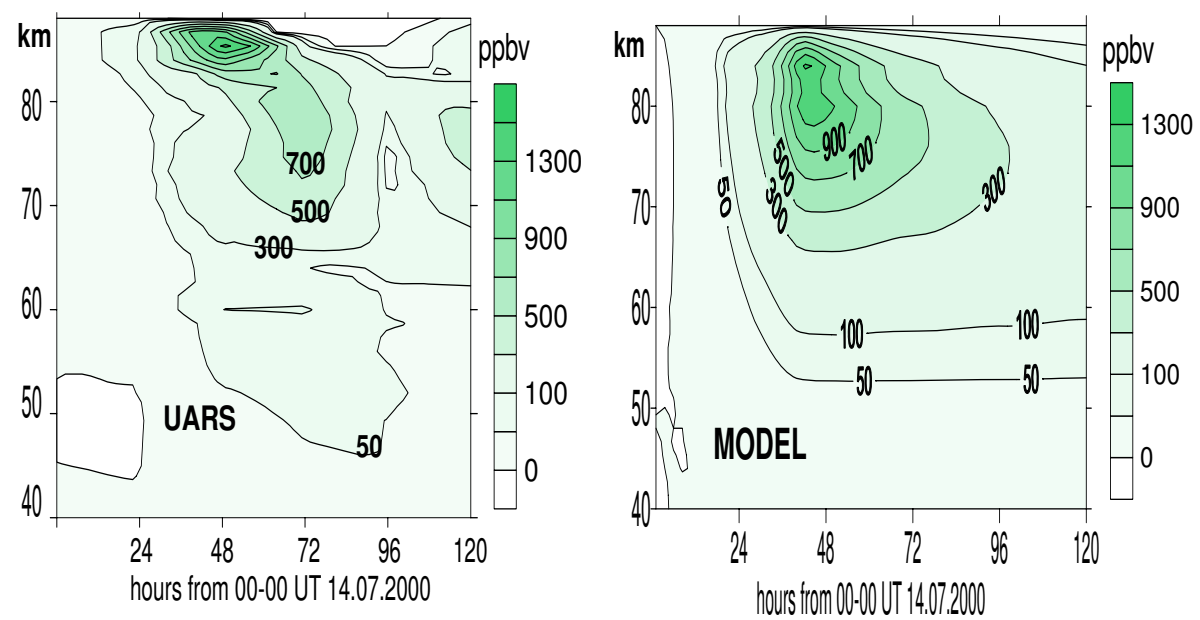

Fig. 34 Changes in $[\mathrm{NO}]$ content (ppbv) at $70^{\circ} \mathrm{N}$ in comparison to non-disturbed conditions of 13th July 2000 revealed from UARS observations (left) and obtained by photochemical simulations. Figure is modified from Krivolutsky et al. (2008).
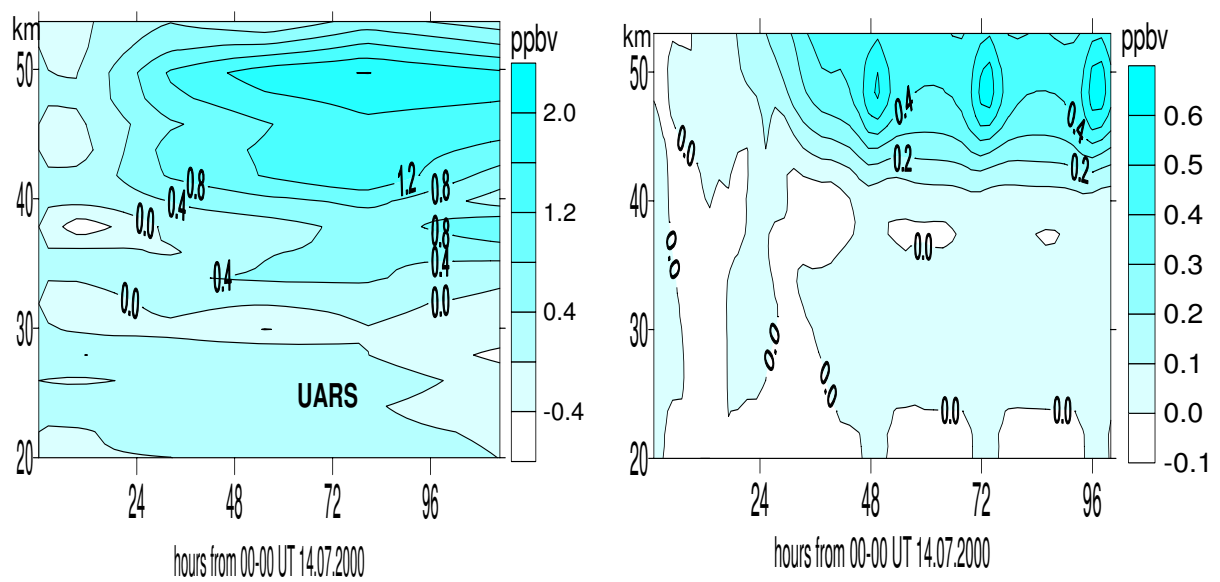

Fig. 35 Changes in $\left[\mathrm{NO}_{2}\right]$ content (ppbv) at $70^{\circ} \mathrm{N}$ in comparison to non-disturb conditions of 13 th July 2000 revealed from UARS observations (left) and obtained by photochemical simulations.

to $\mathrm{NO}_{y}$ in the southern polar region, with $6 \%$ and $11 \%$ contribution, respectively, in the northern polar region.

Some of the latest SEP events (at the time of paper preparation), for cycle 24, have taken place during 23-30th January and 7-11th March 2012. The latest of these SEPs became a GLE event, see Section 2.1.4. These two SEPs were investigated by Jackman et al. (2014). The SEP events lead to significant effects on the chemistry of the middle atmosphere with impacts on mesospheric $\mathrm{HO}_{x}, \mathrm{NO}_{x}$, as well as on $\mathrm{O}_{3}$. The Aura Microwave Limb Sounder (MLS) observations of $\mathrm{HO}_{2}$ displayed enhancements up to $1 \mathrm{ppbv}$ due to the January 2012 SEPs period and up to $0.8 \mathrm{ppbv}$ due to the March 

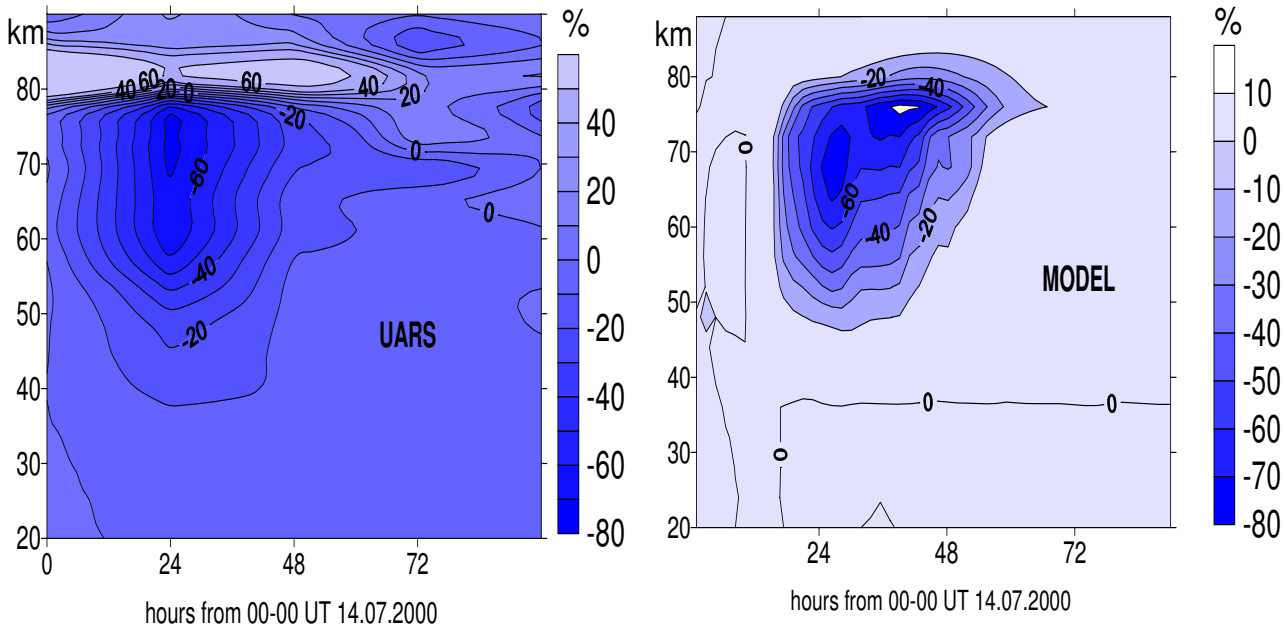

Fig. 36 Changes in $\left[\mathrm{O}_{3}\right]$ content $(\%)$ at $70^{\circ} \mathrm{N}$ in comparison to quiet conditions of 13 th July 2000 revealed from UARS observations (left) and obtained by photochemical modeling. Figure is modified from Krivolutsky et al. (2008).

2012 SEP events period in the Northern polar mesosphere. The Goddard Space Flight Center (GSFC) 2D atmospheric model and Global Modeling Initiative (GMI) threedimensional (3D) chemistry and transport model (CTM) were used to predict mediumterm impacts (months) from the January and March 2012 events. Stratospheric ozone was impacted in the polar regions by these solar events due to the transport of solar energetic particles produced $\mathrm{NO}_{y}$ to lower altitudes. The downward transport moved $\mathrm{NO}_{y}$ produced by the SEPs to lower altitudes and led to predicted modest destruction of ozone (5-13\%) in the upper stratosphere days to weeks after the March 2012 event. Polar total ozone reductions were predicted to be a maximum of $1.5 \%$ in 2012 due to these SEPs.

A large number of simulations as well as observations of atmospheric changes of $\mathrm{HO}_{x}$ and $\mathrm{NO}_{x}$ caused by SEPs have been performed using models of different complexity (e.g., Rozanov et al. 2012; Funke et al. 2011; Krivolutsky et al. 2008; Jackman et al. 2014). It should be mentioned that there are rather few direct observations of $\mathrm{HO}_{x}$ increase exist, since $\mathrm{NO}_{x}$ species and ozone are much more readily observable than $\mathrm{HO}_{x}$ species. The productions of $\mathrm{NO}_{x}$ and $\mathrm{HO}_{x}$ by GCR is still under confirmation by observation due to the small magnitude of the enhancement in the polar upper troposphere/lower stratosphere region, where the effects of GCRs should be the most pronounced (Rozanov et al. 2012). In spite of this some model studies have been already performed, see explanation below.

\section{Galactic cosmic rays}

The influence of GCRs on atmospheric ozone has been investigated mostly using models of different complexity. Recently, the influence of the ionization by GCR on the atmosphere has been quantified using the results of several numerical experiments with the 
Chemistry-Climate models CMAM (Canadian Model of the Middle Atmosphere) (Semeniuk et al. 2011), and SOCOL (Calisto et al. 2011). The ionization rates from GCR in both models were calculated using the state-of-the-art parameterization by Usoskin et al. (2010) (see also Section 2). The numerical experiments were carried out with similar transient set-up, when models were ran for about 30 years in transient mode with the ionization by GCR switched off (reference run) and on (experiment run). The results are presented in Fig. 37 and Fig. 38 as the relative difference of zonal mean $\mathrm{NO}_{x}$ and ozone concentrations between experiment and control runs.

The pattern of the $\mathrm{NO}_{x}$ response to the ionization of the atmosphere by GCR is similar in the both models and consists of the $\mathrm{NO}_{x}$ enhancement in the troposphere and lower stratosphere induced by the $\mathrm{NO}_{x}$ production by GCR. The magnitude and statistical significance of the $\mathrm{NO}_{x}$ response are substantially different. The $\mathrm{NO}_{x}$ enhancement simulated with the CCM SOCOL reaches $20 \%$ and is significant mostly in the southern troposphere and tropical upper troposphere, while CMAM results reveal statistically significant $\mathrm{NO}_{x}$ increase in the entire troposphere/lower stratosphere reaching up to $100 \%$. The reasons for such a substantial disagreement between the simulated $\mathrm{NO}_{x}$ responses are not clear yet. It may be explained by different background $\mathrm{NO}_{x}$ fields in the troposphere and lower stratosphere. The absence of anthropogenic and natural $\mathrm{NO}_{x}$ emissions together with oversimplified tropospheric chemistry in CMAM (Semeniuk et al. 2011) could lead to very small background $\mathrm{NO}_{x}$ abundance and strong impact of the GCR induced source. This hypothesis is partially supported by closer agreement in the troposphere over the southern high latitudes, where the influence of anthropogenic and natural sources of $\mathrm{NO}_{x}$ is the smallest.

The agreement of the patterns and different magnitude/significance of the ozone responses simulated with CMAM and SOCOL models is also evident. The enhancement of the $\mathrm{NO}_{x}$ concentration by GCR activates ozone production via "photo-smog" reactions (e.g., Wang et al. 1998). The effectiveness of the ozone production by additional $\mathrm{NO}_{x}$ strongly depends on the background $\mathrm{NO}_{x}$ field. In the $\mathrm{NO}_{x}$-poor environment the ozone production can be very large, while for the relatively high level of $\mathrm{NO}_{x}$ the ozone production by additional $\mathrm{NO}_{x}$ is limited. Presumably the low background $\mathrm{NO}_{x}$ mixing ratio in CMAM model is the reason of the large (up to $15 \%$ ) ozone enhancement in the entire troposphere, while in the CCM SOCOL significant ozone response is confined to the relatively clean southern hemisphere and reaches only $2-3 \%$. The ozone depletion in the polar lower stratosphere is simulated by both models with much better agreement of the magnitude and location. The ozone depletion is driven by the enhancement of the ozone oxidation cycles caused by additional $\mathrm{NO}_{x}$ and $\mathrm{HO}_{x}$ produced by GCR and similar treatment of the stratospheric chemistry by both models leads to similar pattern and magnitude of the ozone response. The presented results illustrate the upper limit of GCR effects because in the reference runs the ionization by GCR is completely eliminated. Therefore, these effects cannot be observed, however the 11-cycle variability in the GCR intensity over the polar areas (see Section 2) can reach $50 \%$, therefore it would be possible to find 11-year cycle signal in tropospheric $\mathrm{NO}_{x}$ over southern high latitudes if long term time series of proper measurements are available. Another alternative is to analyze the response of $\mathrm{NO}_{x}$ to short-term events such as Forbush decreases. 


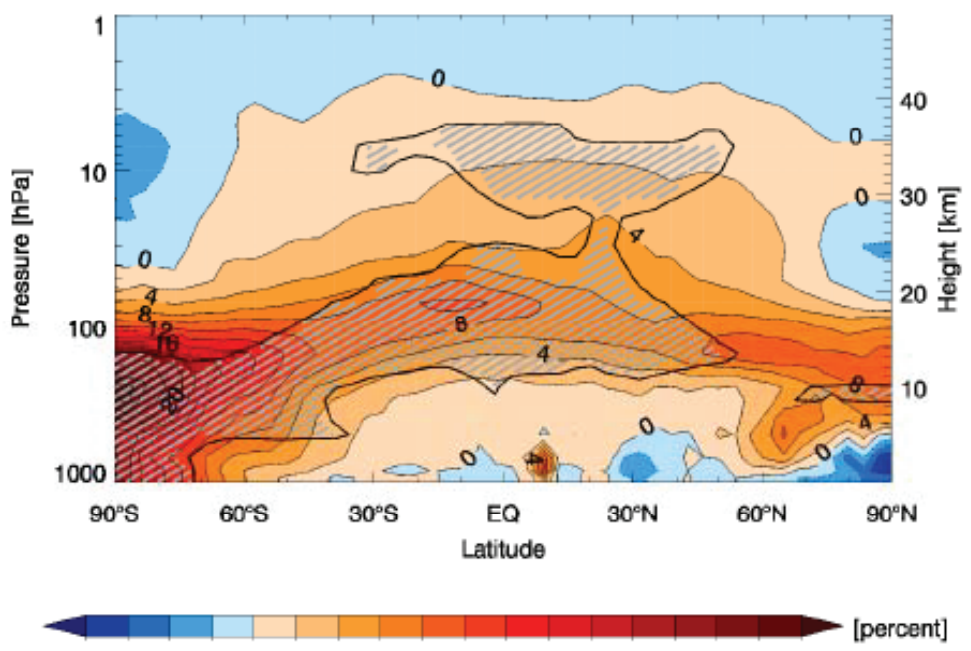

$\begin{array}{llllllllllllllllll}-8 & -6 & -4 & -2 & 0 & 2 & 4 & 6 & 8 & 10 & 12 & 14 & 16 & 18 & 20 & 22 & 24 & 26\end{array}$

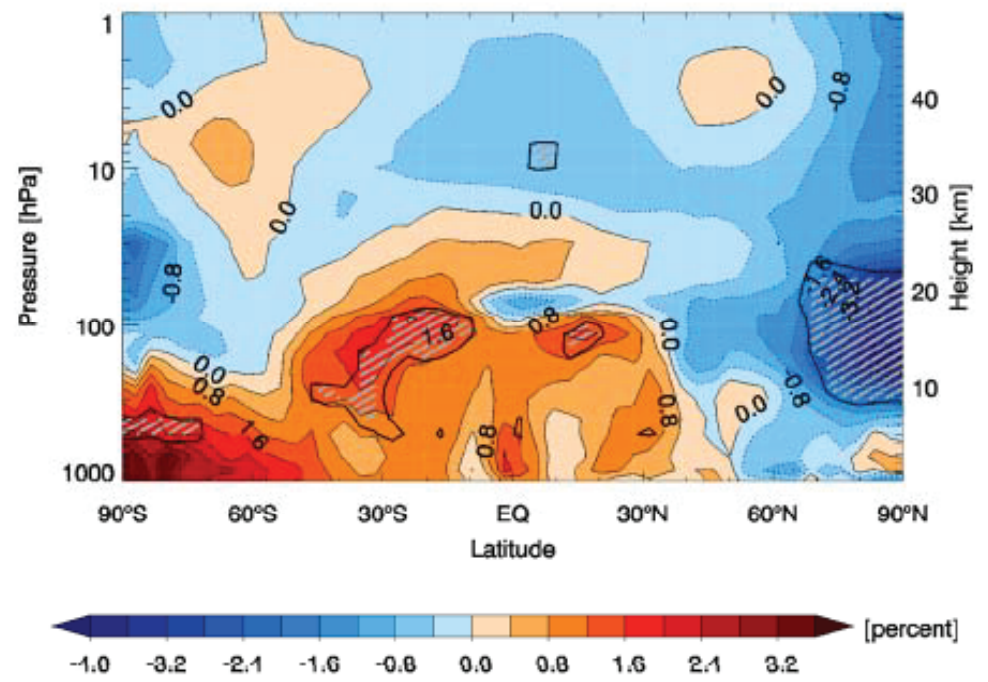

Fig. 37 The simulated annual mean zonal mean $\mathrm{NO}_{x}$ (upper panel,\%) and ozone (lower panel,\%) response to the ionization of the atmosphere by GCR. Hatched areas indicate changes with at least $95 \%$ statistical significance. The results are from Calisto et al. (2011).

\subsection{Atmospheric electricity}

\subsubsection{Introduction to the global electric circuit}

Electrification of the lower atmosphere results directly from ionization from GCRs, and near the surface, also from Earth's natural radioactivity. Ionization occurs when an electron is separated from a molecule, leaving it positively charged, e.g., $\mathrm{N}_{2}^{+}$. The 
NOx
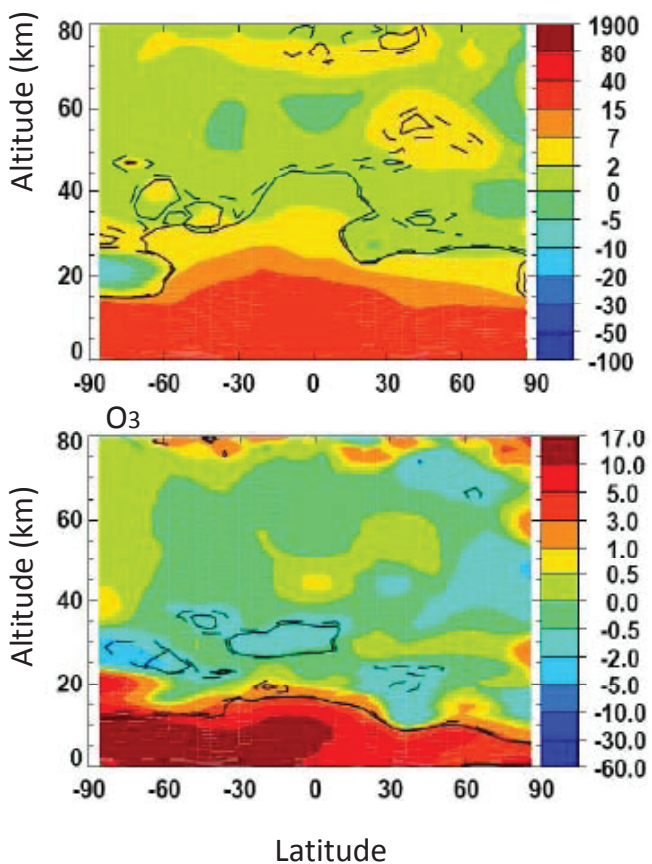

NOx

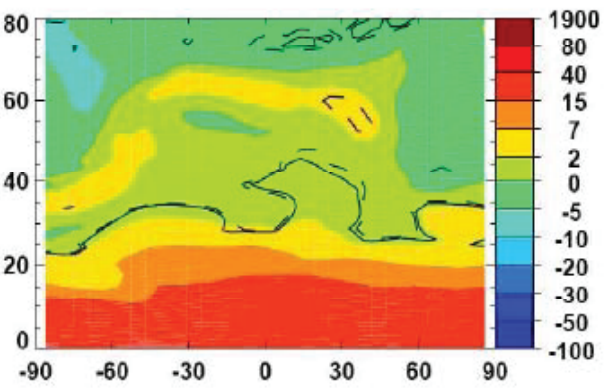

$\mathrm{O} 3$

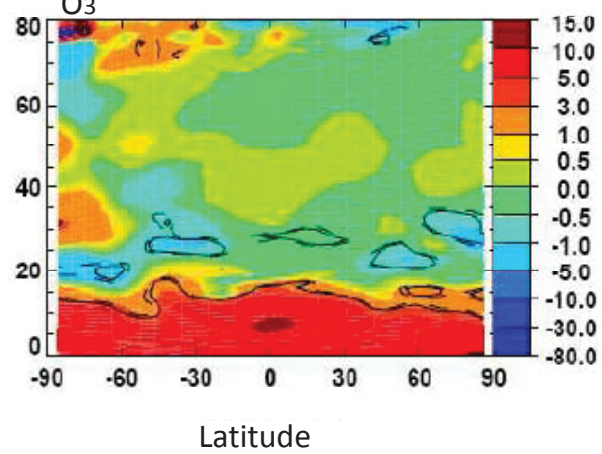

Fig. 38 The simulated zonal mean $\mathrm{NO}_{x}$ (upper panel, \%) and ozone (lower panel, \%) response to the ionization of the atmosphere by GCR. The areas inside solid lines indicate changes with at least 95\% statistical significance. The results are from Semeniuk et al. (2011) for boreal winter (left column) and boreal summer (right column).

electron is then quickly captured by electrophilic molecules, creating a negative ion, e.g., $\mathrm{O}_{2}^{-}$. The primary ions stabilize through clustering with other polar molecules such as ammonia, water, and organics, and are then known as cluster ions (Hoppel et al. 1986).

The presence of bipolar cluster ions gives rise to the electrical conductivity, $\sigma$, of the atmosphere. This finite conductivity permits a small vertical current density, $\mathrm{J}_{z}$, to flow between the conducting regions of the ionosphere and Earth's surface, due to the large potential difference, $\mathrm{V}_{I}(250 \mathrm{kV})$ between them. This potential difference occurs due to charge transfer mainly from 1000 thunderstorms active around the globe at any instant, in a framework known as the global electric circuit (GEC) (e.g., Wilson 1920; Roble 1986). Fig. 39 shows the various processes involved in generation (i.e. thunderstorms and electrified shower clouds) and maintenance (ionization processes) of the circuit.

A fair weather vertical electric field is developed from the currents flowing in the ionospheric potential difference. This electric field is usually referred to as the potential gradient $(\mathrm{PG})$ so that it is positive in fair weather. The vertical conduction current density is related to the ionospheric potential by the columnar resistance, $\mathrm{R}_{c}$, the total resistance of a unit area air column from the ionosphere to the surface

$$
J_{z}=\frac{V_{I}}{R_{c}},
$$




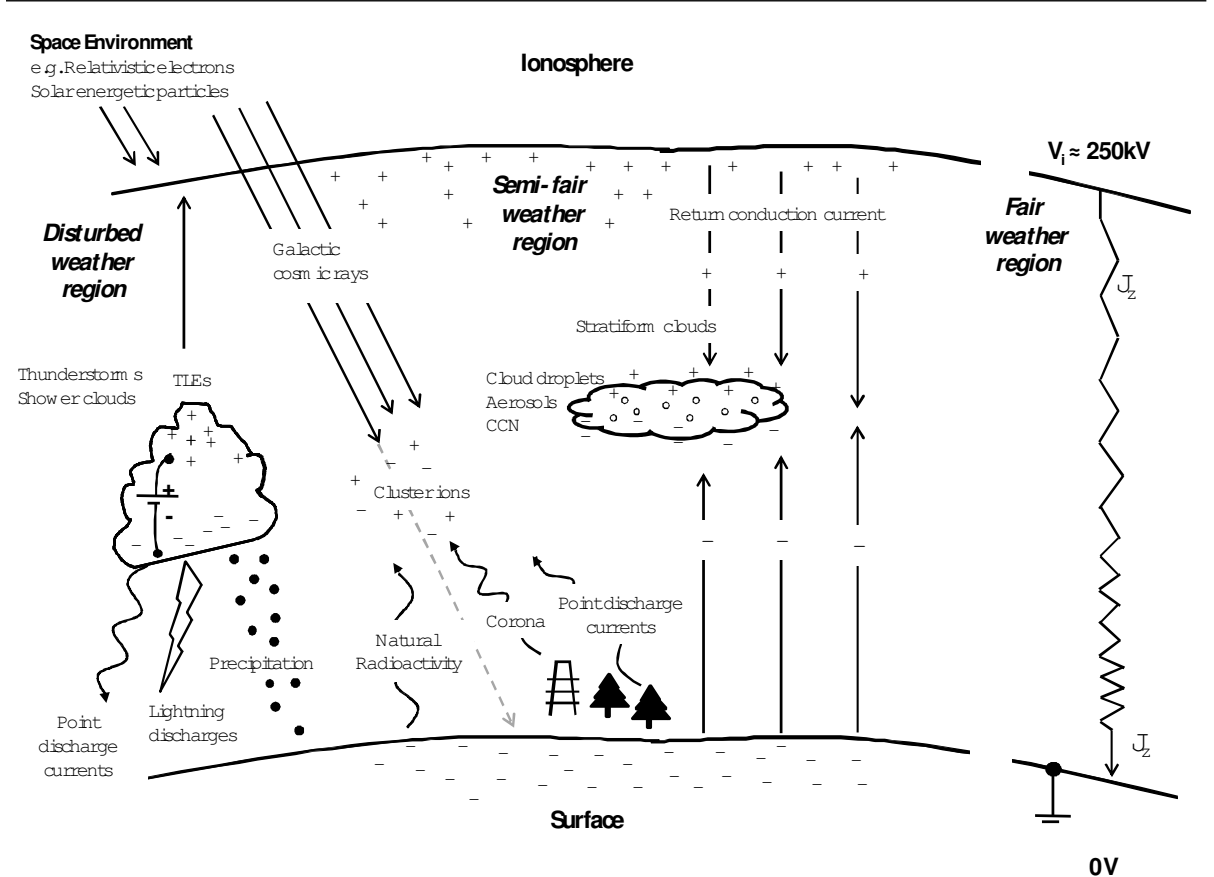

Fig. 39 Atmospheric charge generation and dispersal, including important processes in the global electric circuit, after Rycroft et al. (2012), with permission from Elsevier. Charge separation in thunderstorms creates a substantial potential difference between the highly conducting regions of the ionosphere and the surface. The positive potential of the ionosphere (with respect to the Earth's surface) is distributed to fair weather and semi-fair weather regions, where a small current (with density $\mathrm{J}_{z}$ ) flows vertically. When this current flows through clouds it generates charge near the upper and lower cloud edges, which can influence cloud microphysical processes. (TLEs are Transient Luminous Events (e.g. sprites and jets), cloud condensation nuclei are shown as $\mathrm{CCN}$ ).

$\mathrm{R}_{c}$ can be found by integrating the resistance of atmospheric layers from the surface to the conductive the ionosphere, and is given by

$$
R_{c}=\int_{0}^{\infty} \frac{d z}{\sigma(z)}
$$

where $\sigma(z)$ is the air conductivity at altitude $z$ (Gish 1944). Most of the contribution to $\mathrm{R}_{c}$ is from the lowest $5 \mathrm{~km}$ of the atmosphere, where the air conductivity is reduced by ion loss to aerosol.

\subsubsection{Global electric circuit variability}

Variability in the GEC can result from modulation of any of the parameters in Eq. 18. The major effects are from changes in the global charging current to the ionosphere, but changes in the global conductivity/columnar resistance profiles can also be significant. These processes act over timescales from seconds to years. One of the most widely studied variations in the GEC is the daily variation (Harrison 2013), which is due 
to a diurnal variation in the global charging current to the ionosphere, caused by thunderstorm and electrified shower cloud activity. This variation manifests itself as a minimum at $0400 \mathrm{UT}$ and maximum at $1800 \mathrm{UT}$, and is evident in $\mathrm{V}_{I}$ and $\mathrm{J}_{z}$ data, as well as surface measurements of electric field. This diurnal variation, often referred to as the Carnegie curve, was first detected from measurements made by the geophysical survey ship Carnegie in the early 20th century, the PG measurements from which showed the same diurnal variation irrespective of the location of the ship, providing support for the global nature of the electric circuit (Torreson et al.).

Near the geomagnetic poles there is an additional diurnal variation in GEC parameters, caused by interaction of the solar magnetic field and the geomagnetic field. This generates an electric field, creating an additional potential distribution which is superimposed on $\mathrm{V}_{I}$. During geomagnetically quiet periods, the potentials on the dawn side of the polar cap are 20-30 kV higher than on the dusk side, which increases to a difference of 150-200 kV during geomagnetically disturbed periods (Boyle et al. 1997). This effect generally only extends to within 30 degrees of the north and south geomagnetic poles.

The Carnegie and dawn-dusk polar cap diurnal variations in GEC parameters result from a change in the global charging current to the ionosphere, however, changes can also occur due to variations in the conductivity profiles. Conductivity increases with height from the surface, due to the increase in GCR ionization with height (up to a maximum at $\sim 15 \mathrm{~km}$ ). Since GCRs are deflected by the geomagnetic field, the GCR ionization rate, and therefore conductivity varies with magnetic latitude, with maximum $\sigma$ near the geomagnetic poles, and minimum near the equator ( $\mathrm{Hu}$ and Holzworth 1996). Changes in the vertical profile of conductivity result from variations in the ionization rate (i.e. from GCR flux, SEPs, relativistic electrons), and the presence of aerosol particles (e.g. from volcanic eruptions) and clouds, which decrease conductivity by ion-aerosol attachment.

The global electric circuit acts to couple the space environment with the lower atmosphere, as discussed in Roble (1985) and Rycroft et al. (2012). Energetic particle (EP) effects on the global circuit can therefore indirectly impact the atmosphere. In particular, $\mathrm{J}_{z}$ can have effects on clouds as it flows through and around them (see Section 3.4). The effect of energetic particles on the fair weather part of the circuit will be discussed in detail below, as well as the possibility of energetic particles modulating the charging part of the circuit by triggering lightning.

\subsubsection{Effect of energetic particles on the global electric circuit}

The global atmospheric electric circuit is driven by different atmospheric processes as well as by energetic particles of extraterrestrial origin. There are some evidences that magnetospheric electrons can indirectly affect electrical processes in atmosphere (Rycroft et al. 2012), however the main driver is CRs of galactic and solar origins. As GCRs entering the Earth's atmosphere are the main source of ionization above the surface, both periodic and transient GCR variations are expected to modulate the strength of the global circuit, as outlined below. 


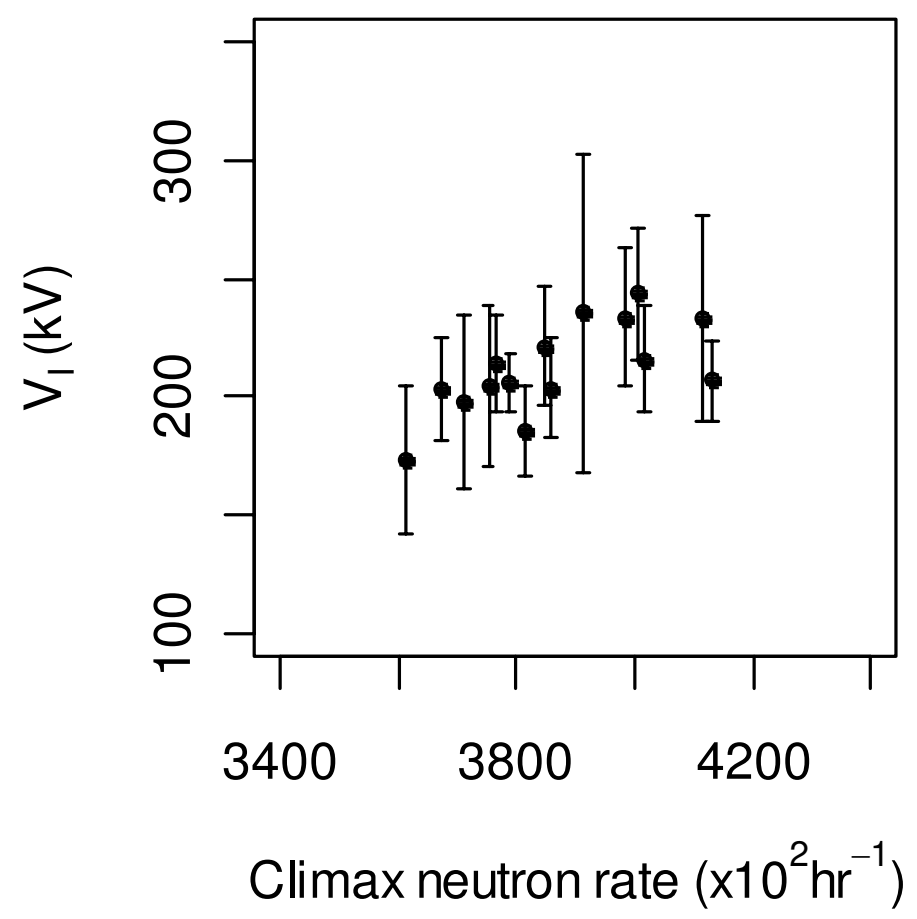

Fig. 40 Comparison of Climax neutron counter data with (a) ionospheric potential $\left(\mathrm{V}_{I}\right)$ measured from 1966-1972 by Mülheisen (Mühleisen 1977), for months having 4 or more $\mathrm{V}_{I}$ measurements available (error bars represent two standard errors). Reprinted from Rycroft et al. (2012), with permission from Elsevier.

\section{Periodic variations}

Markson (1978) suggested that GCRs would reduce the columnar resistance over thunderclouds, increasing the global circuit charging current from lightning. Positive correlations between ionospheric potential and neutron counter measurements of CRs during the late 1960s and early 1970s Mühleisen (1977); Markson and Muir (1980) were used to support this argument. The data (Mühleisen 1977) is summarised in Fig. 40 which shows a positive sensitivity of $15 \%$ in $\mathrm{V}_{I}$ (measured by Mühleisen (1977)) to a $10 \%$ increase in neutron count rate measured from Climax, Colorado. Similarly, Markson (1981) found a $22 \%$ increase in $\mathrm{V}_{I}$ for a $10 \%$ increase in the Mt. Washington neutron rate.

In addition to the observed solar cycle variation in $\mathrm{V}_{I}$, there is evidence of solar modulation of $\mathrm{J}_{z}$. Markson and Muir (1980) reported a $30 \%$ solar cycle variation in $\mathrm{J}_{z}$ in phase with the GCR variation, whilst Harrison and Usoskin (2010) found $\mathrm{J}_{z}$ at Lerwick, Shetland, to be $17 \%$ lower, and the PG to be $12 \%$ lower during solar maximum.

As almost all of the columnar resistance originates from lower tropospheric aerosol, it is difficult to see how GCRs could have any appreciable effect on the upper troposphere columnar resistance. Harrison and Usoskin (2010) argue that GCRs reduce the columnar resistance everywhere, rather than just over thunderclouds. The reduced 
columnar resistance therefore affects the conduction current density and potential gradient via the "fair weather" (non-thunderstorm) part of the global atmospheric electric circuit. Combined with the geomagnetic change in cosmic ray ionization, it is likely that the combination of source term (thunderstorm current generation), load term (columnar resistance), and load term spatial variation (geomagnetism) all contribute to the observed changes.

\section{Transient energetic particle effects}

Short term irregular variations in the solar output also perturb the GEC. Solar flares, which release large amounts of energy from the sun, accelerate solar energetic particles (SEPs) to high speeds, which interact with Earth's atmosphere via the geomagnetic field. SEPs access to the atmosphere is restricted by magnetic rigidity cutoff, in a similar way to GCRs, see Bazilevskaya (2005) and Section 2, whereby SEPs are generally restricted to polar regions. SEPs create an additional source of ionization in the stratosphere, perturbing the vertical conductivity profile and local electric field at high geomagnetic latitude (e.g., Holzworth and Mozer 1979). The largest SEP emissions produce ground level event enhancements (GLE) where the secondary energetic particles produced by $\mathrm{GeV}$ protons can be detected by surface cosmic ray detectors away from the poles.

Coronal Mass Ejections (CMEs) are usually associated with solar flares, which transport the sun's magnetic field, and often arrive at Earth several days after the parent flare. CME's create magnetic disturbances, as well as often intensifying the magnetic barrier to GCRs, causing a decrease in the incoming GCR flux, known as a Forbush decrease. Forbush decreases usually have an onset time of a day or so, with a slow recovery lasting about a week. They generally result in a decrease in the cosmic ray induces ionization (CRII) rate measured by neutron monitors of $10 \%$ (although larger events, up to $30 \%$ have occurred). Their effects can reach down to the mid latitudes.

The time difference between the arrival at Earth of SEPs from a solar flare and the CME shock impact can be several days, potentially allowing attribution of certain GEC changes to particular processes, however the additional influence of geomagnetic storms, which can occur throughout the duration of the event, and alter the geomagnetic cutoff energies for GCR penetration complicate matters somewhat. Such magnetic variability has been shown to perturb the ionospheric potential, $\mathrm{V}_{I}$ at high latitudes as well as the surface electric field, even down to mid latitude locations (e.g., Olson 1971; Kleimenova et al. 2008).

\subsubsection{Surface measurements}

Evidence of short term solar effects on the GEC can be seen in surface measurements of atmospheric electric parameters, but only when measurements are made during fair weather conditions, from sites that are not polluted. This is essential as local influences such as adverse weather conditions for observations (e.g. fog, cloud, precipitation, high winds) can mask the subtle signals generated by solar induced changes in the GEC. Such observations have been made by Cobb (1967) $\left(20^{\circ} \mathrm{N}\right.$ geomagnetic latitude) and Reiter (1969) (40 $\mathrm{N}$ geomagnetic latitude) who showed electric field and/or $\mathrm{J}_{z}$ changes at mountain observatories associated with solar flares. Using data for 28 SEPs and a 

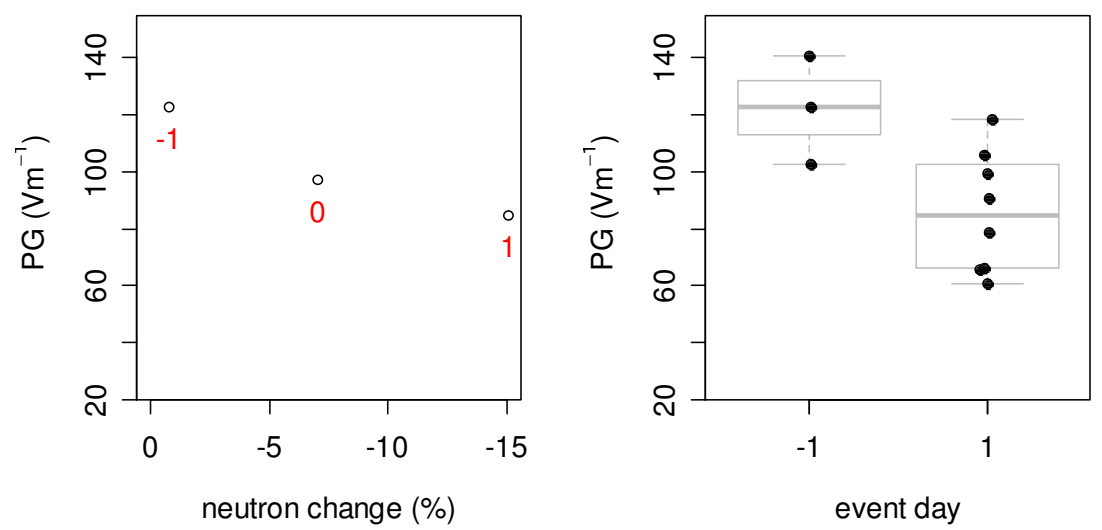

Fig. 41 (a) Nagycenk mean PG (values 1 UT to 5 UT only, with at least 3 hourly values between 40 and $200 \mathrm{~V} / \mathrm{m}$ ), composited on Forbush changes $10 \%$ or greater (inter-event gap at least 5 days) at the Oulu neutron monitor, for the day before ("day -1") or after ("day 1") the event day (day 0).(b) The measurements used in (a) are presented as individual values, but with a summarising box plot overlain, in which the thick line represents the median PG and the boxes give the inter-quartile range.

superposed epoch analysis, Cobb (1967) found an increase in $\mathrm{J}_{z}$ of up to $11 \%$ on the first day following the flare. Similar results were found by Reiter (1969) from the Zugspitze in Germany, who observed increases in $\mathrm{J}_{z}$ of about $20 \%$ following large solar flares. Although an effect on atmospheric electrical parameters is evident from these data sets, the responses are to a mixture of events including SEP events, Forbush decreases and geomagnetic activity.

Changes in surface electric field due to short term solar influences are also evident from the measurements of Märcz (1997) who demonstrated a decrease in PG in Hungary (geomagnetic latitude $47^{\circ} \mathrm{N}$ ) following Forbush events, using a superposed epoch analysis technique. This is illustrated in Fig. 41.

Conversely, measurements of $\mathrm{PG}$ and $\mathrm{J}_{z}$ from several mid latitude stations compiled by Sheftel et al. (1994) during six Forbush events both showed an increase immediately following the onset of the Forbush decrease. The largest increase in PG and $\mathrm{J}_{z}$ occurred at the minimum point in the Forbush event, when the GCR ionization rate was lowest. Provided Eq. 18 holds, the simultaneous increase in PG and $\mathrm{J}_{z}$ in the presence of the expected decrease in $\sigma$ appears to indicate an increase in $\mathrm{V}_{I}$ during these measurements.

The inconsistent results discussed above demonstrate the difficulty of detecting a small solar signal in atmospheric electric field data, which can be readily dominated by local effects. Another approach would be to analyse the effects of GLEs from surface-based instruments, but the number of events available for analysis is small (Bazilevskaya 2005), before exclusion of meteorological effects and quasi-coincident Forbush decreases. 


\subsubsection{Airborne measurements}

Although evidence of solar influences on atmospheric electric parameters can be seen from the surface, detection is easier at higher altitudes where signals are larger, well above cloud systems or other sources of complicating variability. Such measurements have been made from a high altitude balloon flight (Holzworth et al. 1987) during an SEP event, which detected a decrease in the magnitude of electric field and increase in $\sigma$, where both polarities of conductivity were enhanced by a factor of two (see Fig. 42). Ionization was also measured during the same balloon flight (altitude $26 \mathrm{~km}, 44.6^{\circ} \mathrm{S}$, $142.7^{\circ} \mathrm{E}$ ), with count rates increasing from $\approx 30$ to 100 counts per minute, coincident with the SEP event. A second balloon at similar altitude but different geomagnetic latitude $\left(38.7^{\circ} \mathrm{S}, 65.7^{\circ} \mathrm{E}\right)$ showed no detectable change in electric field and $\sigma$, allowing the spatial extent of the SEP event on the electrical environment to be determined. Thus the rigidity cutoff at the poleward balloon enabled SEPs to perturb the electrical environment, but not at lower latitudes. Similar measurements at high altitudes during SEP events have also been made by Holzworth and Mozer (1979), whose observations during the August 1972 SEP event agreed well with model calculations of the same event by Reagan et al. (1983) and Kokorowski et al. (2006), who also observed increases in local $\sigma$ and reductions in electric field.

The high altitude balloon flights described above provide long duration measurements at constant altitude, however, free balloons are required to obtain a vertical profile of conditions throughout the atmosphere, such as the balloon measurements by Gringel (1978) which demonstrate how the vertical conductivity profile changes from the surface up to $30 \mathrm{~km}$ in the days following a solar flare. Figure 43(a) shows measurements made on the 8th August 1972 from Germany after a period of intense solar flares on the 2nd, 4th and 7th of August. The observed reduction in conductivity compared to normal background levels was most likely due to the intense Forbush decrease in the GCR flux (20\% at Oulu, Finland), which began on 4th August and was still in the recovery phase during the period of balloon measurement. A similar reduction in conductivity was also observed during a balloon flight from Germany on 14th April 1978 following a Forbush decrease on 10th April (Fig. 43(b)). Such balloon measurements have been instrumental in demonstrating that local conductivity can be both increased (due to extra ionization from SEPs), as well as decreased (from reduction in the GCR flux during a Forbush event), depending on geomagnetic latitude and altitude.

Changes in $\mathrm{J}_{z}$ have also been observed to occur during solar flare events, as found by the balloon measurements of Cobb (Cobb 1978) from the Antarctic. From a series of regular balloon ascents (one a day for five consecutive days), Cobb observed an increase in $\mathrm{J}_{z}$ above altitudes of $25 \mathrm{~km}$ approximately 17 hours after a solar flare event. During the following day, the entire $\mathrm{J}_{z}$ profile was enhanced by $70 \%$ above pre-flare conditions, with a return to pre-flare conditions three days after the flare onset. These measurements are in agreement with model calculations by Reagan et al. (1983) who found that following a large SEP event, the current density carried by solar protons and electrons can greatly exceed that carried by $\mathrm{J}_{z}$ at high altitudes $(>15 \mathrm{~km})$, but only within the polar caps. Thus it is likely that the increase in $\mathrm{J}_{z}$ observed by Cobb (1978) was a response to the additional locally generated current created by enhanced ionization at high altitudes.

Although the effects of SEP's on atmospheric electrical parameters can be significant at high altitudes and at high latitudes, the magnitude of their effects on the global electric circuit are questionable. As suggested by Holzworth and Mozer (1979) 


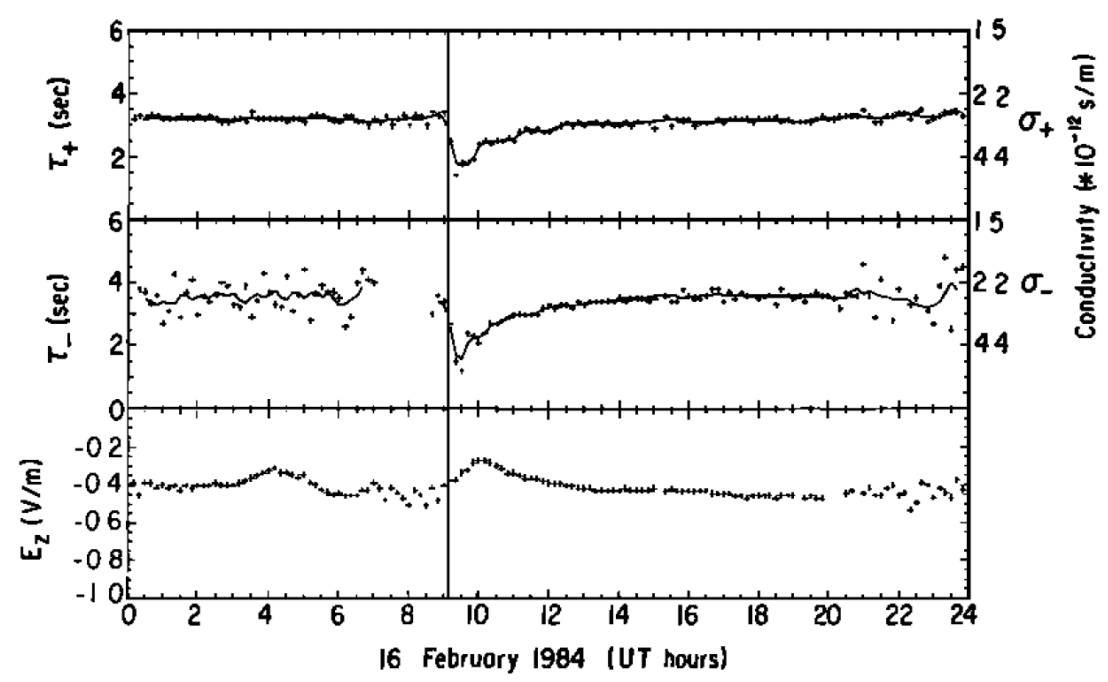

Fig. 42 Measurements of atmospheric electrical parameters from a high altitude balloon at $26 \mathrm{~km}$ during a SEP event on 16 th February $\left.1984,44.6^{\circ} \mathrm{S}, 142.7^{\circ} \mathrm{E}\right)$. Positive and negative relaxation time constants $(\tau+$ and $\tau$-) are shown on the upper left-hand axis, with corresponding polar conductivities on the upper right-hand axis. Vertical electric field, $\mathrm{E}_{z}$ is shown on the lower left-hand axis. The vertical line at 0910UT denotes the start of the solar energetic particle event. Reprinted from Holzworth et al. (1987), with permission from Wiley.
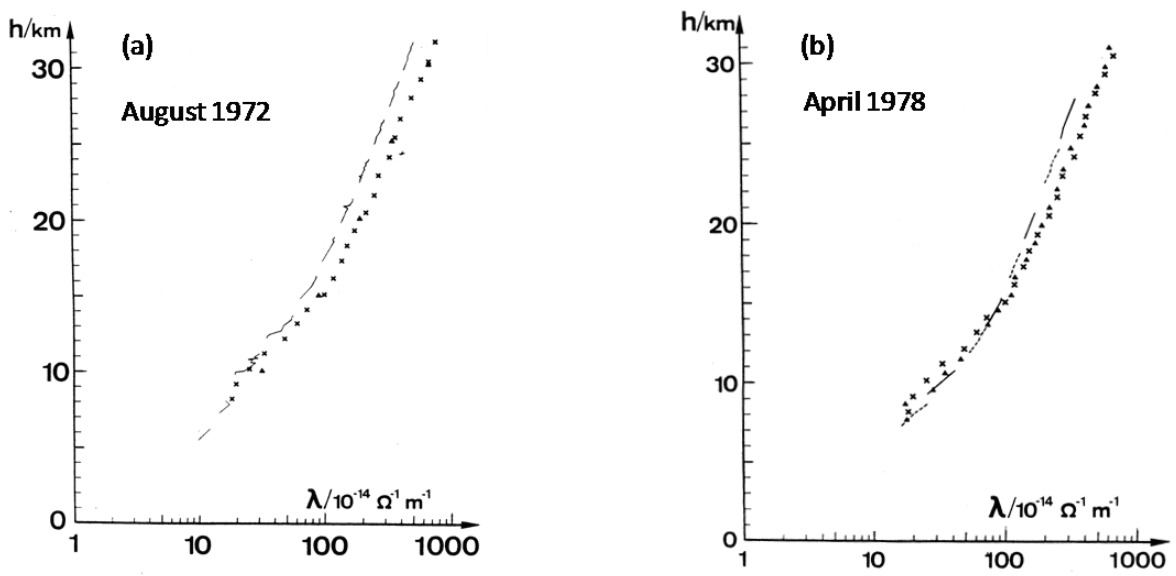

Fig. 43 (a) Balloon profile of positive conductivity measured on 8th August 1972 (solid line) after solar flares on 2nd, 4th and 7th August 1972, compared to profiles measured during quite sun conditions (crosses and triangles). (b) Balloon profiles of positive and negative conductivity measured during quite and active solar conditions. Lines represent active solar conditions, measured on 14th April 1978 after a series of solar flares on the 10th and 11th April 1978 (solid line positive conductivity, dashed line negative conductivity), whilst shapes represent quiet solar conditions (crosses positive and triangles negative conductivity). All profiles were measured over Germany. Taken from Gringel (1978). 
and supported by model simulations of the August 1972 SEP event by Reagan et al. (1983) and Tzur et al. (1983), the largest changes in GEC parameters (10\% in $\mathrm{V}_{I}, \mathrm{~J}_{z}$ and $\mathrm{PG}$ ) are related to the change in GCR flux during the Forbush decrease, with little effect of the SEP event on the GEC.

One coupling mechanism that could be influenced by SEP events is the response of the thunderstorm generator part of the GEC. The GEC current is dependent on stratospheric/mesospheric resistance above a thunderstorm, which varies with ionization rate (Markson 1978). Increases in ionization rate from SEPs penetrating to low latitudes ( $>$ several hundred $\mathrm{MeVs}$ ) can potentially decrease the resistance above thunderstorms sufficiently to increase the upward charging current to the ionosphere. Model calculations by Farrell and Desch (2002) find that during large SEP events, this can occur, in turn affecting $\mathrm{J}_{z}$ as well as the surface electric field, however, changes in PG for even the biggest SEP events are only expected to be of the order of $5 \%$.

\subsubsection{GCR and lightning}

Energetic GCR may directly trigger lightning through a mechanism known as "runaway breakdown", when electrons are accelerated to relativistic energies by a high electric field, and lose some of their energy by ionization (and also Bremsstrahlung, when gamma-rays are emitted which are detected as "Terrestrial Gamma Ray Flashes"). If the electrons generated are energetic enough, then an electron avalanche develops leading to breakdown (Roussel-Dupré et al. 2008). This mechanism needs a single electron of energy $1-10 \mathrm{keV}$ to start the breakdown, which can be provided by GCR. The electric fields needed to start runaway breakdown are an order of magnitude lower than the $2 \mathrm{MVm}^{-1}$ needed to initiate conventional breakdown. This, and the paucity of observations of adequate electric fields in thunderclouds for conventional breakdown, has led to the suggestion that lightning is triggered by electrons from GCR initiating runaway breakdown (Roussel-Dupré et al. 2008). Further evidence for the existence of runaway breakdown is provided by the numerous recent observations of energetic photons from thunderstorms, which are predicted by runaway breakdown theory (Gurevich and Zybin 2005).

If this mechanism is a significant source of lightning, there should be a positive relationship between GCRs and lightning. However, studies investigating this effect are difficult and often inconclusive due to the high levels of natural variability in thunderstorm occurrence and lightning flash rates (Schlegel et al. 2001). There are also difficulties with obtaining reliable and consistently calibrated lightning data. In a study using 16 years of lightning data over the USA, Chronis (2009) found that lightning activity dropped 4-5 days after a Forbush decrease in GCRs. He also identified a positive correlation between lightning and GCRs during the winter. Although these effects are of the right sign to support a GCR-lightning triggering mechanism, clearly more work is needed, in particular to understand the timescales involved.

In summary, although evidence of a solar influence on atmospheric electrical parameters exists, the solar-electrical coupling mechanisms are not well understood. Over longer timescales such as the 11-year solar cycle, the sign of the relationship between cosmic ray ionization, $\mathrm{R}_{c}$ and $\mathrm{J}_{z}$ is as expected from theory. The magnitude of the effect and the exact mechanisms acting are not as well understood. GCRs ionization changes by $10 \%$ over a solar cycle, which, based on a simple consideration of Eq. 18, 
would lead to a $5 \%$ drop in columnar resistance. The work reported in Section 3.3 above shows that the response is several times larger than expected, implying the GEC is acting as an amplifier. More data is needed at different geomagnetic latitudes to corroborate this finding, but if it is global, one explanation for the amplification could be that GCR affect both the "fair weather" and "disturbed weather" parts of the global circuit. In the first case this is through generation of conduction currents, and in the other, through direct triggering of lightning.

On shorter timescales, EPs generate a multitude of effects, of which the atmospheric electrical responses can be divided into several components:

1. Response to increased SEP flux. This increases ionization, usually only at high latitudes. GLEs are a subset of the strongest SEP events, that precipitate particles down to the surface.

2. Response to Forbush decrease in GCRs flux. This decreases ionization globally.

3. Response to precipitation of particles from the radiation belts during geomagnetic storms.

4. Change in GCRs cutoff rigidity during geomagnetic storms. This may only be a locally detectable effect.

5. Downward mapping of ionospheric electric fields associated with solar wind/magnetosphere dynamo, generally only at high latitudes.

Each of these components has its own distinctive features, and every event is a complicated superposition of these disturbances. Local effects add further variability, although they can be averaged or minimised with careful data analysis. To more fully understand the effects of solar influences on the GEC, more measurements of atmospheric electrical responses to short term solar perturbations are required, with high temporal resolution measurements, over a wide range of geomagnetic latitudes. Further work is required to understand the role of changes in the source term (thunderstorm current generation), load term (columnar resistance), and load term spatial variation (geomagnetism) all of which are likely to contribute to the observed changes in atmospheric electrical parameters. 


\subsection{Clouds}

\subsubsection{Mesospheric clouds}

The clouds occurring at the greatest heights in the atmosphere are mesospheric clouds, at about $80 \mathrm{~km}$. They consist of ice, and their presence depends on the water vapour present despite the ultra-low humidity environment of the mesosphere. For the ice particles to nucleate, the atmospheric conditions must be very cold, which means they are most common in polar regions, and when the mesosphere is coldest. Some mesospheric clouds become visible from the surface at mid- to high latitudes after sunset, when they are illuminated by sunlight from below the horizon, and are known as noctilucent clouds (NLC). NLC nucleate initially in the summer mesopause, sedimenting until they dissipate by sublimation. NLC were probably first observed from the late eighteenth century (Fogle and Haurwitz 1966) perhaps in 1885 or 1886 (Dalin et al. 2012), which, despite changes in observing practices, therefore provides some long term information on their occurrence. Satellite observations for up to about the past 30 years have also become available (Shettle et al. 2009) which utilise ultraviolet backscatter for detection of polar mesospheric clouds.

Figure 44 shows a 43 year time series of the number of nights during which NLC were observed from the UK and Denmark, as tabulated by Kirkwood et al. (2008). For both the total NLC incidence and that of the brightest nights, an approximately 11-year cycle is evident. This cycle is in antiphase with the solar cycle, as observed using the $10.7 \mathrm{~cm}$ wavelength solar radio noise (Fig. 44b). This variation has been explained as resulting from breakdown of water molecules by the ultraviolet (EUV) component of the solar radiation. This is supported by the anti-correlation between polar mesospheric clouds and the solar Lyman-alpha irradiance (DeLand et al. 2003). Hence, during solar maximum when EUV flux is greatest, much reduced molecular water is available and NLC become rarer. Kirkwood et al. (2008) report a lag of 13-17 months between NLC occurrence maxima and the $10.7 \mathrm{~cm}$ solar radio flux, and find that, by allowing for this and the duration of the summer conditions, that $40 \%$ of the year to year variation in NLC numbers can be explained. The study of satellite data from 1978-1992 of DeLand et al. (2003) found a 0.5 year phase lag for Northern Hemisphere polar mesospheric cloud data, but no lag in the Southern Hemisphere.

In the context of energetic particle effects, however, the anti-phase variation of NLC occurrence with the solar cycle indicates an in-phase variation with galactic cosmic rays. Figure 44 accordingly shows the Oulu neutron counter variation during the same time interval. The possibility of aerosol formation from cosmogenic ions at $90 \mathrm{~km}$ has previously been suggested (Witt 1969), which has some support from the short-timescale of NLC disappearance associated with geomagnetic disturbance (Arnold 1980a). Distinguishing between cosmic ray and solar ultra-violet effects within atmospheric variability is a general problem confronting the understanding solar-influenced processes in the troposphere (Harrison and Tammet 2008). This may be aided by the new range of parameters encountered from the decrease in solar activity currently occurring (Lockwood et al. 2011). A more direct effect of energetic particles is now understood to arise from solar proton events (SPEs), during which the NLC occurrence rate decreases. For example, a reduction in the NLC occurrence rate exceeding the background variability was observed during SPE of January 2005 (von Savigny et al. 2007). The NLC reduction is now understood to result from an associated increase in mesospheric temperatures (Winkler et al. 2012). 
(a)

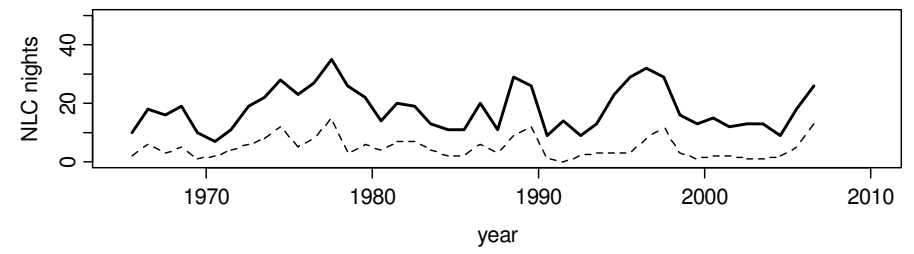

(b)

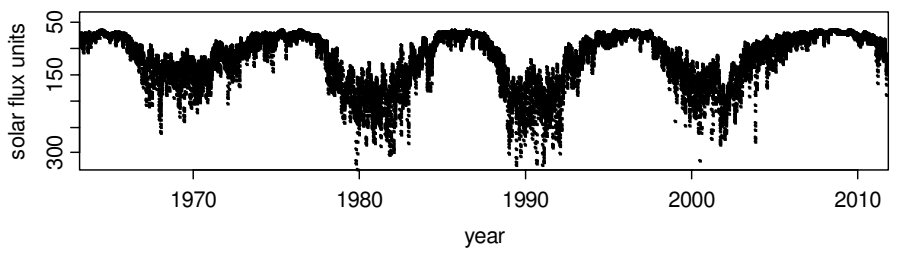

(c)

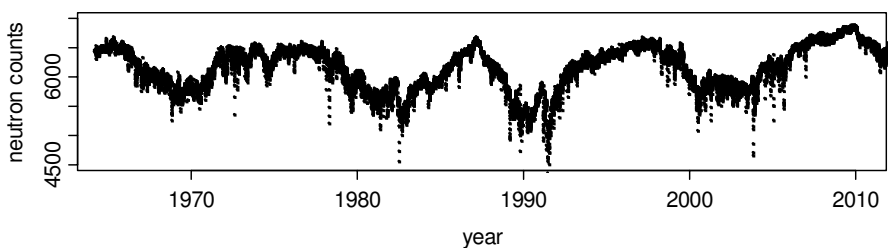

Fig. 44 (a) Number of nights annually on which noctilucent clouds (NLC) were observed over the UK (excluding northern Ireland) or Denmark (Kirkwood et al. 2008), with the number of nights classified as "bright" marked with the dashed line. (b) Solar radio output at $10.7 \mathrm{~cm}$ (in solar flux units, $1 \mathrm{SFU}=10^{-22} \mathrm{~m}^{-2} \mathrm{~Hz}^{-1}$ ), and (c) Oulu neutron counts, for the same interval.

\subsubsection{Stratospheric clouds}

The absence of appreciable water vapour in the stratosphere largely prevents cloud formation, but thin, nacreous clouds, which are almost invisible from the surface do form as a result of local cooling from upward-propagating gravity waves or flow over mountains. The synoptic-scale of polar stratospheric clouds can fill essentially the whole polar vortex area. Stratospheric clouds at the poles, however, receive close attention, because of their role in generating active chlorine which leads to ozone depletion. Polar stratospheric clouds (PSC) are classified according to their composition: PSC Type 1 contain nitric acid and in some cases sulphuric acid as well as water or ice, and PSC Type 2 consist solely of ice. Cosmic ray ionization has been suggested to initiate the freezing which forms large nitric acid trihydrate (NAT) particles (Yu 2004), through the reorientation of polar solution molecules into the crystalline configuration with strong electric fields. Polar stratospheric aerosols may also be affected by major solar proton events. It was found that major SEP/GLE events during January 2005 (Mironova et al. 2008, 2012) and during September 1989 (Mironova and Usoskin 2013) may have led to formation of new particles and/or growth of preexisting ultrafine particles in the polar stratospheric region. The extra aerosol mass formed under the decreasing temperature 
allows attributing most of the changes. The continuation of study of SEP/GLE events of July 2000, April 2001 and October 2003 suggests that an enhancement of ionization rate by a factor of about two in the polar region with night/cold/winter conditions can lead to the formation/growing of aerosol particles in the altitude range of 10-25 km (Mironova and Usoskin 2014).

\subsubsection{Troposphere}

Any responses in clouds to energetic particles within the lower troposphere are, in principle, of great interest because of their importance to the planetary radiative balance. Gray et al. (2010) review some of the observational studies which argue for and against this possibility of a CR effect on clouds. In general, various different methods of cloud observation (global satellite, surface radiometers, manual observation or cloud proxies such as the diurnal temperature range) have been compared with neutron counter time series, and statistical relationships extracted (e.g., Marsh and Svensmark 2000). Because solar irradiance and GCRs vary closely together, attention has been focussed on specific signatures unique to cosmic rays, in particular Forbush decreases, in an attempt to study changes dominated by cosmic ray changes. A range of results have been found, such as no global cloud effect (Calogovic et al. 2010), a combination of aerosol and cloud transient responses in response to selected Forbush events (Svensmark et al. 2009) and weak cloud property responses in limited areas (Kristjánsson et al. 2008). As Forbush decreases are rare, many events must be averaged together if their effects are to overcome natural variability in clouds (Harrison and Ambaum 2010). Accompanying some Forbush decreases however, there are also precursory changes in total solar irradiance (Laken et al. 2011), which potentially complicate their conventional interpretation as events unique to cosmic rays. Although on average these changes in irradiance are proportionally small $(\sim 0.03 \%)$ compared with the Forbush decreases, see Fig. 45, some irradiance changes can, individually, be greater, especially for large Forbush decreases. As direct ("Wilson") nucleation of droplets cannot occur at the water super-saturation levels of the troposphere, and a spontaneous freezing response of supercooled water droplets to CRs is not observed under laboratory conditions (Seeley et al. 2001), two physical frameworks have instead been suggested which do offer potentially plausible mechanisms linking cosmic rays and clouds (Carslaw et al. 2002). They consider non-thunderstorm clouds, as thunderstorms are intensively electrified, but only occur over a small fraction of the planet's surface. Smaller effects on the more abundant clouds have been considered to be more likely to generate a global effect. In the first of these mechanisms, (the "clear-air") effect, cosmic ray induced ionization (CRII) is considered to generate new ultrafine aerosol particles, which are assumed to contribute to the formation of clouds as cloud condensation nuclei (CCN), and in the second (the "near-cloud") effect, charging at cloud edges influences cloud microphysical processes and, in turn, cloud properties.

\section{Clear-air effect}

Experimental work continues to investigate the role of ions in the formation of new atmospheric particles at low ionization rates and trace gas concentrations. For example, Enghoff et al. (2011) have used an electron beam to generate ions for nucleation of 
sulphuric acid aerosol, and Kirkby et al. (2011) have shown that surface cosmic ray ionization could substantially increase the nucleation rate of sulphuric acid and sulphuric acid ammonia particles, by two to ten times in some circumstances. Model calculations have not, however, demonstrated an appreciable effect on climate through this mechanism. Kazil et al. (2006) applied the Lovejoy model (Lovejoy et al. 2004) to average ambient conditions appropriate to the troposphere over the oceans. These simulations predicted negligible charged and neutral nucleation of $\mathrm{H}_{2} \mathrm{SO}_{4}$ and $\mathrm{H}_{2} \mathrm{O}$ in the tropical lower troposphere, even in the absence of pre-existing aerosol, but, at mid-latitudes, the charged nucleation exceeded neutral nucleation as long as the pre-existing aerosol concentration was depleted, e.g. following precipitation. Kazil et al. (2006) estimated an upper limit for the change in daily mean shortwave radiative forcing between solar maximum and minimum from charged nucleation cloud cover changes, of $0.24 \mathrm{Wm}^{-2}$. Independently, Pierce and Adams (2009) found less than $0.1 \%$ change in CCN concentrations despite a $15 \%$ change in galactic cosmic rays during a solar cycle, which is found to be equivalent to a change of $5 \mathrm{mWm}^{-2}$ in solar radiation reflected from clouds. Subsequently, Kazil et al. (2012) found asymmetry in hemispheric responses to solar modulation of ions with peak values of $0.14 \mathrm{Wm}^{2}$ in the southern and 0.06 $\mathrm{Wm}^{-2}$ in the northern mid-latitudes, and globally $-0.05 \mathrm{Wm}^{-2}$.

\section{Near-cloud effect}

The small radiative changes predicted from the clear air effect have increased attention on the near-cloud effect, through which droplets on the edges of horizontal layer clouds become electrified from the conduction current flowing in the global atmospheric electric circuit. The amount of charging is proportional to the gradient of droplets concentration and the conduction current density, $\mathrm{J}_{c}$. Observations show that $\mathrm{J}_{c}$ varies with CRII, as increased conductivity from increased cosmic ray ionization facilitates increased current flow; in turn a modulation with cloud edge charging is expected. Measurements support the existence of modest droplet charges, and even within strong updrafts which are expected to reduce the peak charge through collisions, mean droplet charges of $\sim 100$ e (elementary charges) have been observed (Beard et al. 2004). Recently, cloud edge measurements have been made directly using radiosondes (Nicoll and Harrison 2010) and, using such techniques charging is apparent (Rycroft et al. 2012), yielding 10e per droplet. The charge effects on the cloud microphysics are still being investigated, but they divide into effects concerned with particle (or droplet) collection by other droplets, and droplet formation or evaporation. These effects may also be combined, such as the evaporation of highly-charged droplets leaving highlycharged particles with greatly enhanced collision efficiencies with other liquid droplets (Tinsley 2000). In general, charge effects are likely only modifying the behaviour of the smallest droplets and particles, because of the smaller proportional inertial effects. For the first group of mechanisms, theory and experiments show that aerosol charging considerably enhances the collection of aerosol by water droplets (Wang et al. 1978; Tripathi et al. 2006), which, in the case of supercooled water clouds, could increase freezing by enhancing the rate of contact nucleation (Tinsley et al. 2000). For the second mechanism, Harrison and Tammet (2008) suggest that droplet charging facilitates droplet formation by inhibiting evaporation.

Figure 46 summarizes these different mechanisms schematically, and provides an indication of the charge levels required to affect the cloud process concerned, each of 
(a)

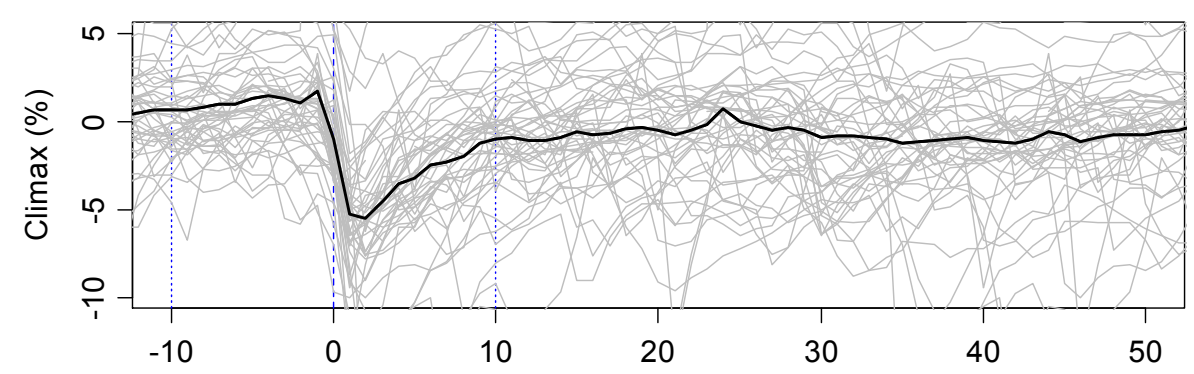

(b)

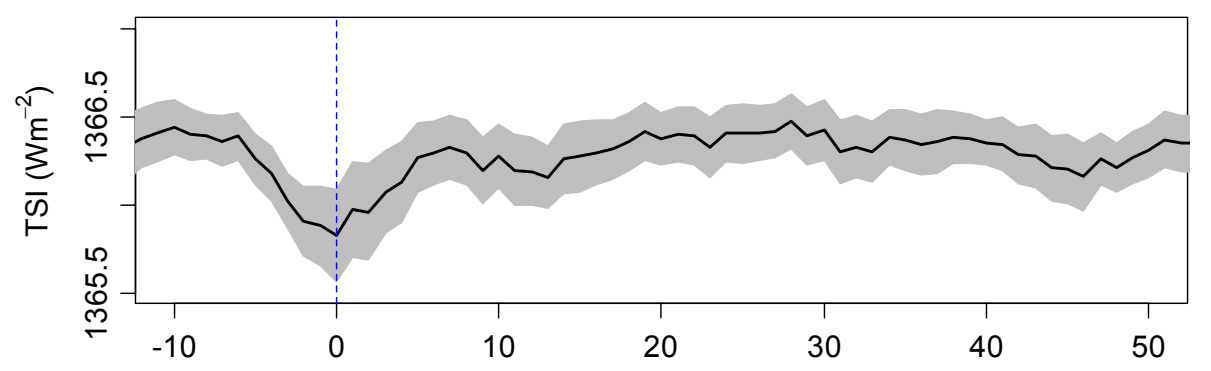

Fig. 45 Composites (thick lines) of changes in Climax neutron counter (a) and (b) the Total Solar Irradiance (TSI) formed on changes of $5 \%$ or greater in the neutron counter data, separated by at least 10 days. In (a) the individual events are shown as grey lines, with the thick line the mean; in (b) the thick line is again the mean, but with the grey band representing 95\% confidence limits on the mean. The TSI data in the figure are from PMOD/WRC, Davos, Switzerland (version 24, (Fröhlich and Lean 1998)) include unpublished data from the VIRGO Experiment on the cooperative ESA/NASA Mission SOHO. The Climax neutron counter was supported by National Science Foundation Grant ATM-9912341.

which is effectively considered in isolation. It should be noted that, in a real cloud, these and many other processes occur simultaneously, in competition, hence this approach is for comparison purposes only. In Fig. 46(a), the charge effect on the droplet evaporation is evaluated. This is presented in terms of critical supersaturation, which expresses the local water vapour concentration needed for a droplet to grow rather than evaporate. If the critical supersaturation is reduced, such as through charging, the droplet becomes stable with a smaller critical supersaturation. It is apparent that, even for the pure droplets considered to maximize the effect, charging of at least 100 e per droplet is required for an effect.

Particle and droplet collection can be appreciably influenced by small amounts of charge as the electrostatic force can dominate over inertial forces at short ranges. Collision efficiency quantifies how readily a collision occurs between a drop (or particle) as it moves in the flow field around another object, by measuring how far off axis the moving particle can begin moving, yet still be captured. Deriving this quantity requires repeated trajectory calculations (Wang et al. 1978; Klimin et al. 1994; Tinsley et al. 2000) to determine the boundary between capture and loss. For collection of charged particles by droplets (Fig. 46b), the collision efficiency with submicron particles can 
(a) Droplet formation

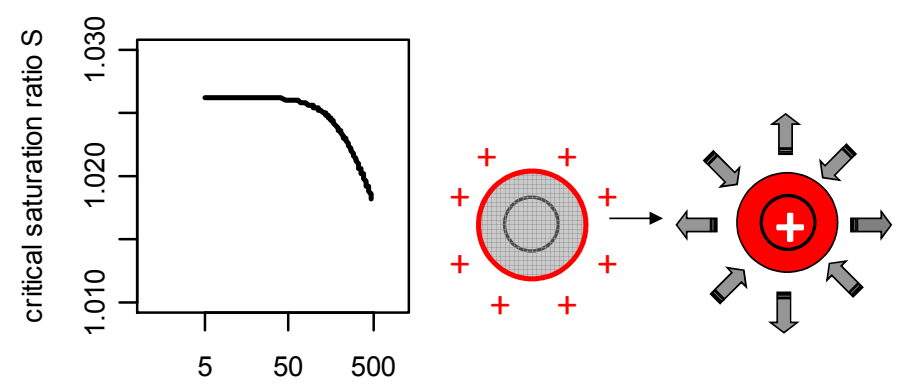

droplet charges (e)

(b) Particle collection

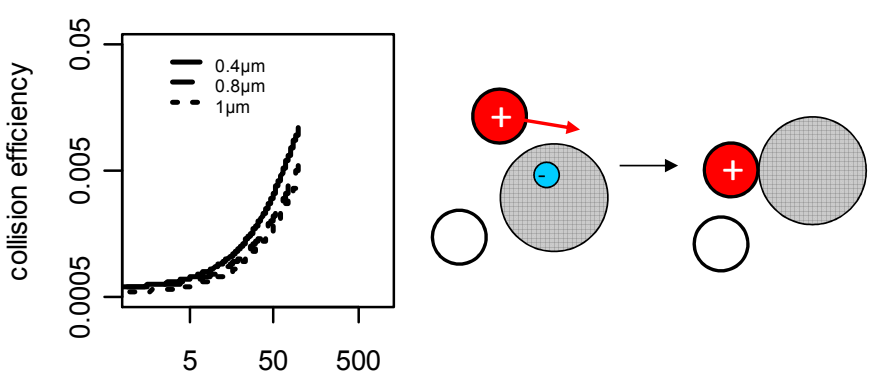

particle charges (e)

(c) Droplet collection

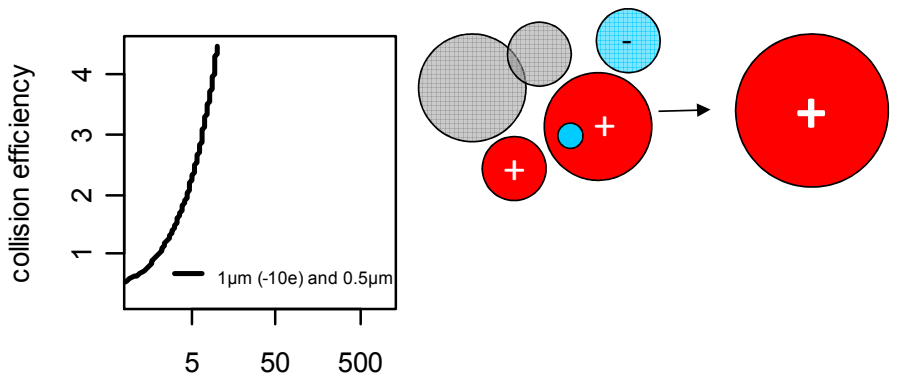

small drop charge $(e)$

Fig. 46 Comparison of selected cloud microphysics processes influenced by small quantities of charge (adapted from Rycroft et al. (2012)), with schematic interactions of neutral droplets (grey), charged droplets (larger circles, blue and red), and charged aerosol (smaller circles, blue and red). (a) Droplet formation. Plot shows variation of critical supersaturation (the supersaturation at which a droplet becomes activated, and grows) with droplet charge, for a pure water droplet containing $2.5 \cdot 10^{-21} \mathrm{~kg}$ of sodium chloride (from Harrison and Ambaum (2009)). (b) Particle collection. Plot shows collision efficiency between a neutral $50 \mathrm{~m}$ radius water drop and a charged particle (density $1.5 \mathrm{gm}^{-3}$ ) for three radii of aerosol carrying variable charge (Tripathi et al. 2006). (c) Droplet collection. Plot shows collision efficiency between opposite polarity water droplets of radii $1 \mu \mathrm{m}$ and $0.5 \mu \mathrm{m}$, the larger carrying 10 elementary charges and the smaller variable charge, in an electric field of magnitude $100 \mathrm{Vm}^{-1}$ (Klimin et al. 1994). 
be increased by about an order of magnitude for $\sim 50$ e per particle. But for dropletdroplet interactions, when both droplets are small ( $\sim$ micron size or smaller $)$, only a few elementary charges are needed. Their collision efficiency is greatly enhanced if the charges on interacting droplets are of opposite signs; the very large collision efficiency values shown result from the formulation of collision efficiency, when droplets are deflected well away from their otherwise independent motions by the electrostatic attraction. These effects fall off rapidly with increasing droplet size. A cloud droplet population model is needed for the electrostatic effects to be evaluated simultaneously with other droplet and particle interactions rather than in isolation, and thereafter to allow the radiative effect on the cloud to be evaluated. Even so, it is clear from these considerations that there are processes which, in principle, can be affected by modest amounts of charge, associated with cosmic influences on atmospheric ionization. 


\section{SUMMARY}

In this section we distil the discussions of the previous sections into tables to summarise the state of our knowledge. These tables attempt to represent the state of knowledge of the different effects of energetic particles on processes throughout the atmosphere. The definitions used in the tables are also summarised after the tables.

SUMMARY Table 2 considers the energetic particles effect on the thermospheric and mesospheric layers of the atmosphere. Here we summarize effects of electrons and SEPs on the chemistry of these layers. The role of auroral electrons as well as SEPs in the chemistry of these layers is well established. However, understanding the role of radiation belt electrons remains unresolved. In the thermosphere, precipitating auroral electrons generate reactive nitrogen, as confirmed by observations and models. Production of $\mathrm{NO}_{x}$ and $\mathrm{HO}_{x}$ by solar proton events is also well-established theoretically and observationally. However production of $\mathrm{HO}_{x}$ is observed only indirectly by the subsequent ozone loss during the event. However, this ozone loss is generally very well reproduced by models including the $\mathrm{HO}_{x}$ production parameterized due to Solomon et al. (1981). The chemical role of radiation belt electrons in the mesosphere is rarely observed and there is also no realistic model study of these effects because the ionization rates required are unknown. Downward propagation of NO produced from energetic particles is observed, but not well understood. Models do not reproduce this propagation quantitatively and the dynamical coupling is not well understood, especially that following a large stratospheric warming. The quantitative amount of stratospheric ozone loss is therefore still uncertain. Downward propagation of NO produced by energetic particles may nevertheless be important for climate, with a potential effect on the polar surface temperature.

SUMMARY Table 3 concentrates on the energetic particle effect on the stratospheric layer of the atmosphere. Effects of electrons, SEPs as well as GCRs on the polar vortex are summarised, together with effects on stratospheric aerosol, polar stratospheric clouds and chemical effects of cosmic rays. The ionization effect of energetic particles mostly occur for regions where ozone destruction leads to cooling of the polar vortex, with the possibility of an effect on the Arctic Oscillation. There may also be an indirect link to climate because of an associated effect on polar surface temperature. Stratospheric aerosol and polar stratospheric clouds can also be affected by ionization generated from major SEPs or GLEs, with associated effects on temperature. In the stratosphere ionization from GCRs is a source of chemical changes. Nitric acid production during polar winters can affect polar clouds and lead to indirect influences on climate.

SUMMARY Tables 4 to 7 provide an assessment of troposphere responses, and specifically that of tropospheric clouds to cosmic rays and cosmogenic ions.

SUMMARY Table 4 concentrates on energetic particles' effects on the tropospheric atmosphere through the direct effect infrared influence on cluster ions. This may generate a radiative effect which is global in extent but currently is unquantified. Some models studies (Calisto et al. 2012; Semeniuk et al. 2011) also show NO production arising from GCRs ionization, which may impact on climate.

Effects of the EPs on atmospheric electricity can occur through cosmic ray triggering of lightning and conductivity changes which affect the global atmospheric electric circuit.

SUMMARY Tables 5 to 7 concentrate on the GCRs and global atmospheric electric circuit effect on clouds, which principally concerns the troposphere, considered 
at length in Section 3.4.3. The theory of GCRs triggering of lightning is well-established, see Section 3.3.6, suggesting a positive relationship between cosmic rays and lightning. However there is not yet robust evidence for this effect - in part because of the short length of the data record from stable lightning detection networks - and some studies are contradictory. A GCR effect on the global atmospheric electric circuit is suggested from evidence on solar cycle and daily timescales. Taken together, the atmospheric electrical pathways may lead to an impact on global production of $\mathrm{NO}_{x}, \mathrm{O}_{3}$ from lightning and through an indirect radiative effect by modulation of clouds. All these effects are, however, mostly contentious, or even controversial, and of unknown magnitude.

\section{Definitions used in the Tables below}

\section{(See SUMMARY Tables 2- 7)}

Well established - theory and observations confirm the presence of the effect quantitatively; Observed - observations support the existence of an effect; Expected from theory - accepted theory predicts the effect; Probable - Theory or physical reasoning is likely to explain a hypothesized or observed effect; Possible - Theory or physical reasoning indicates there should be an effect, without confirmatory observations; Suggested - postulated concept which lacks detailed theory or confirmatory observations; Contentious - constructively discussed effect under active debate; Controversial - strongly differing opinions held which are yet to be resolved; Unlikely - theory or physical reasoning yields a low likelihood of an effect occurring; Not known - investigations from basic theory or observations remain to be made. 


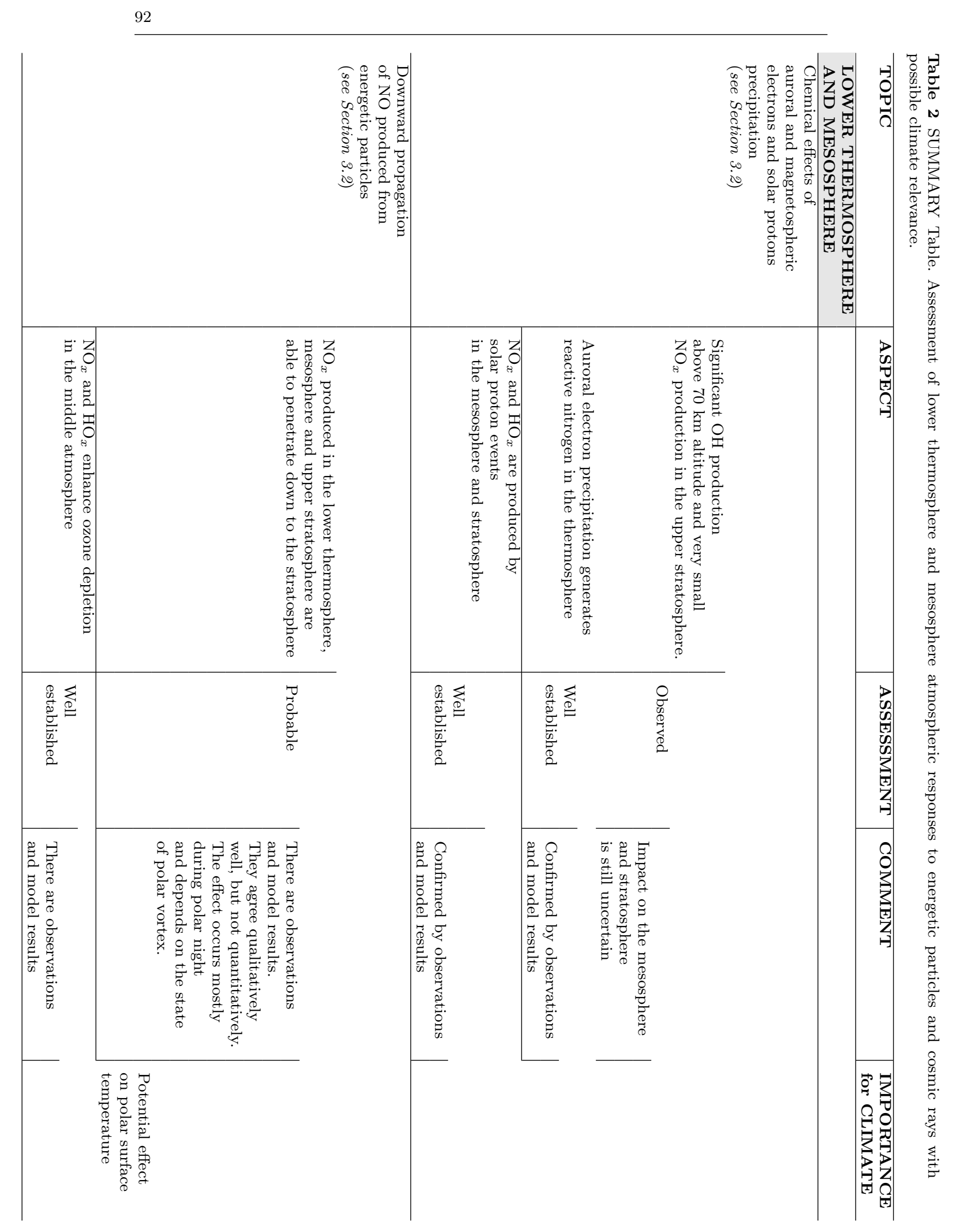




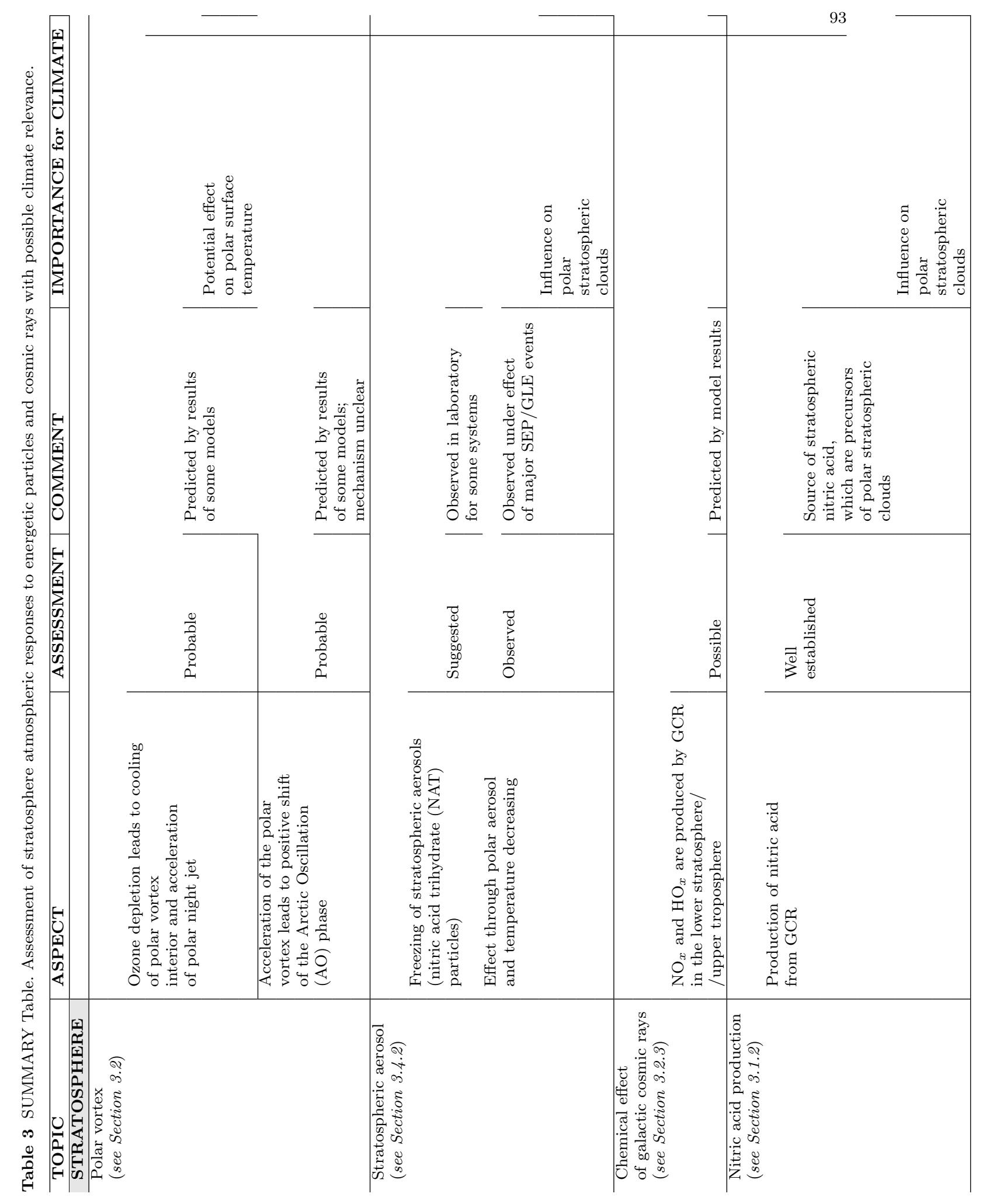




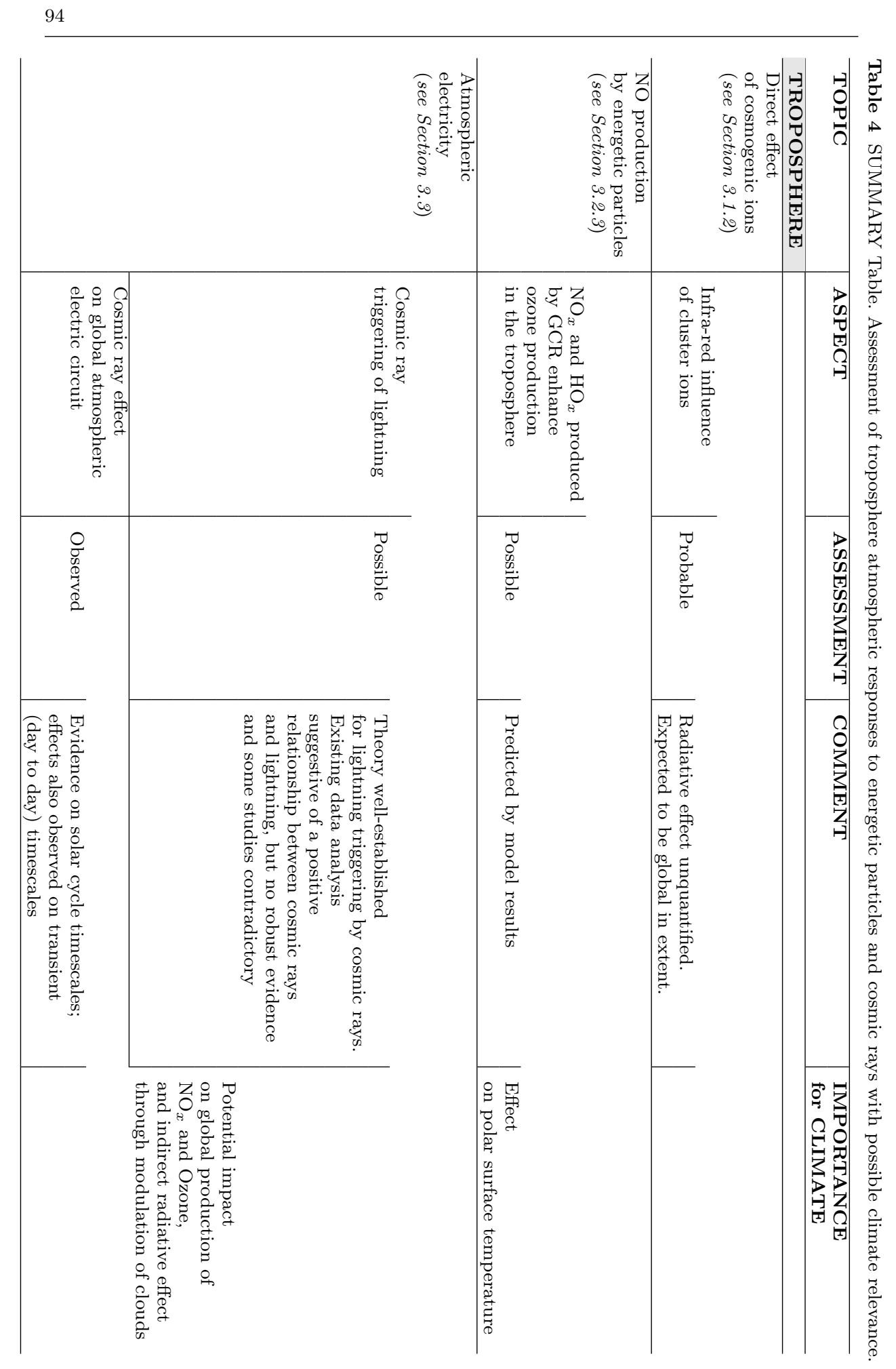




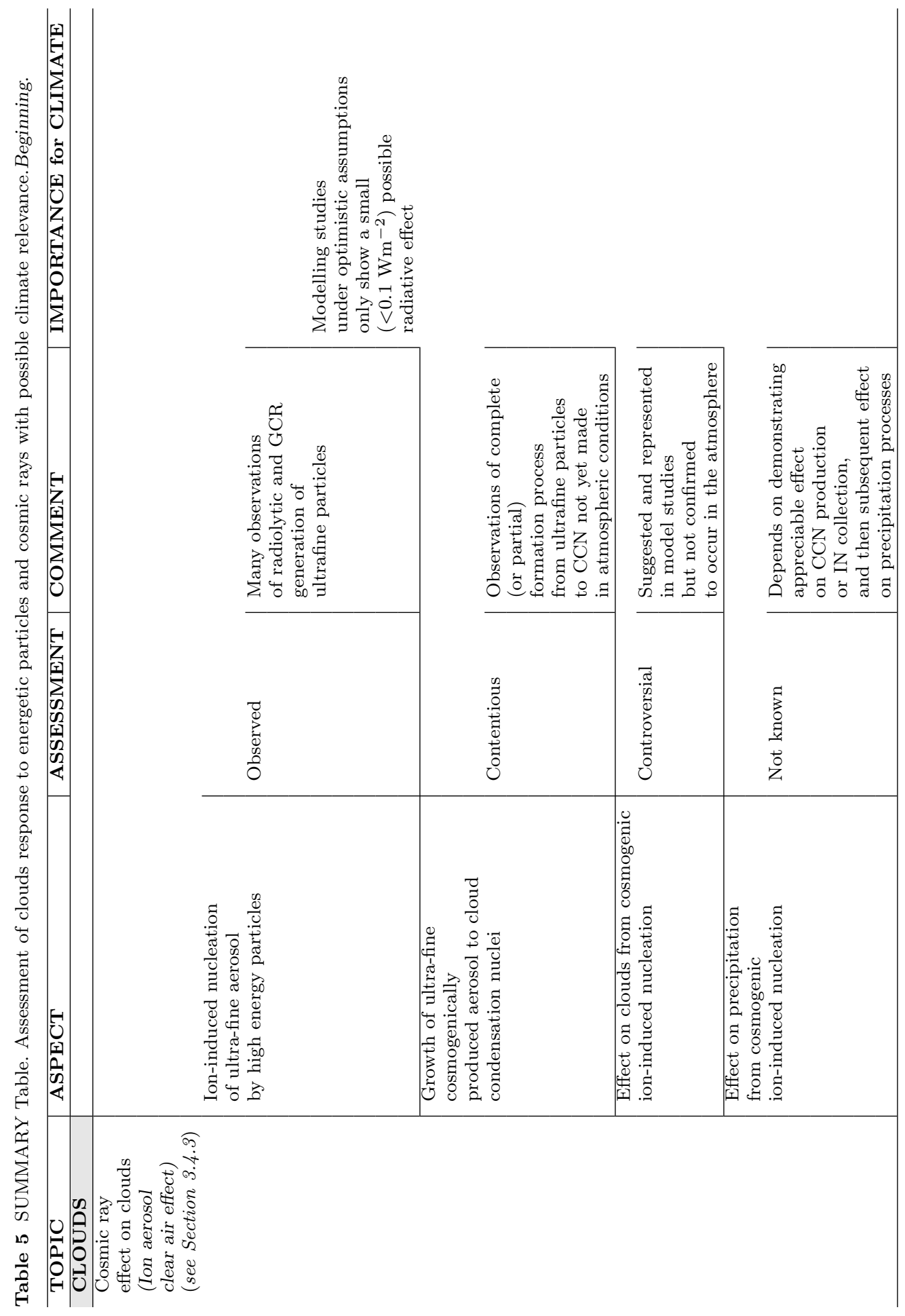




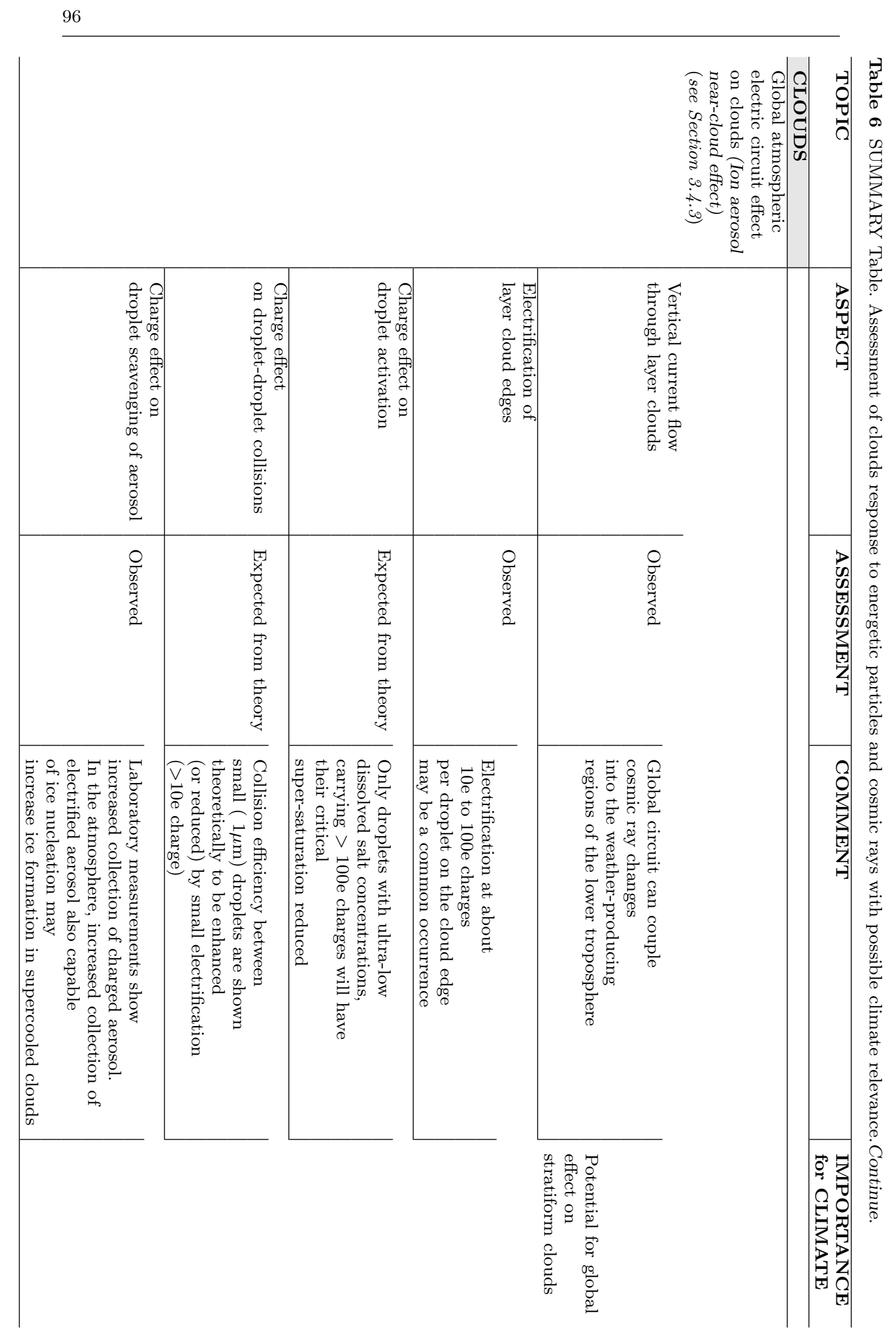




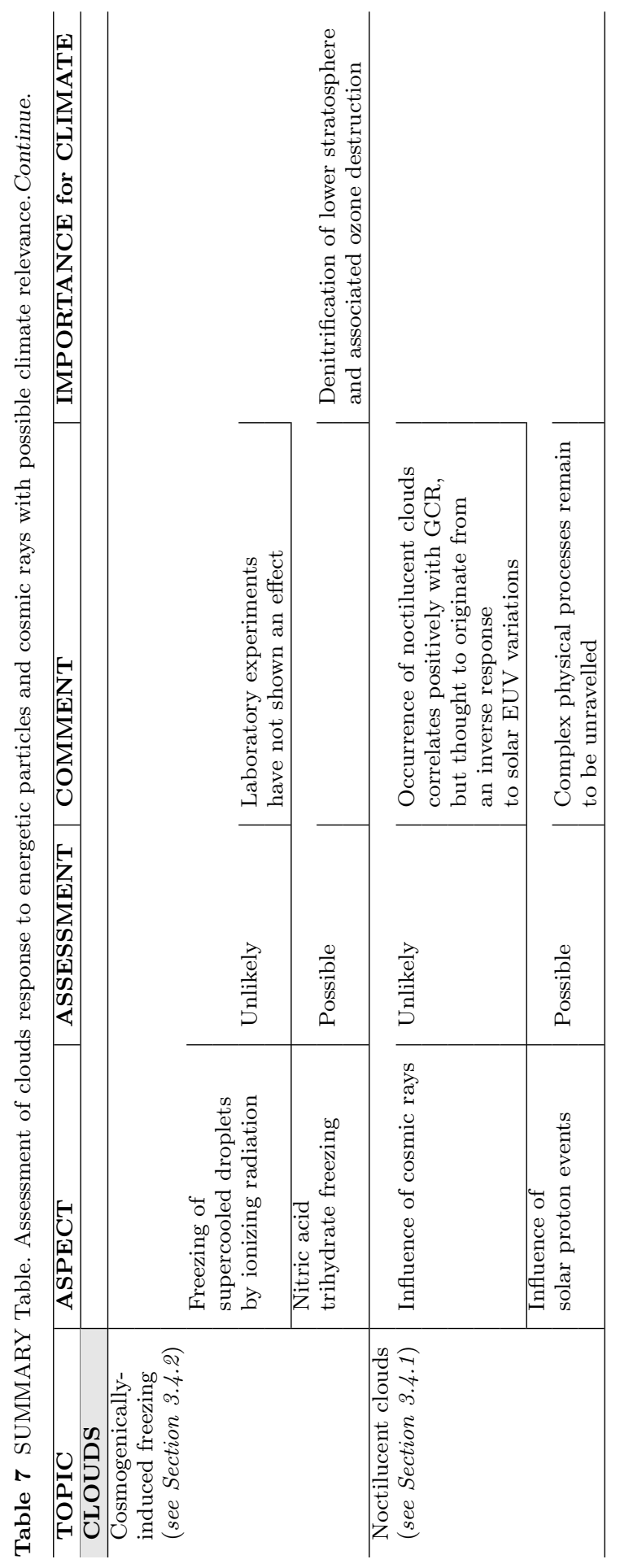


Abbreviations used in the manuscript

ACR - anomalous cosmic ray

$\mathrm{CR}$ - cosmic ray

CRAC - cosmic ray atmospheric cascade

CME - coronal mass ejection

CRII - cosmic rays induced ionization

CIR - corotating interaction region

CCN - cloud condensation nucleair

CCM - chemistry climate model

CGL - corrected geomagnetic latitude

EAS - extensive air showers

EPP - energetic particle precipitation

EPPs - energetic precipitating particles

EP - energetic particle

EEP - energetic electron precipitation

EMIC - electromagnetic ion cyclotron

EUV - extreme ultraviolet

GCR - galactic cosmic ray

GLE - ground level enhancement

GMIR - global merged interaction region

GEC - global electrical circuit

HCS - heliospheric current sheet

IMF - interplanetary magnetic field

$\mathrm{NM}$ - neutron monitor

NLC - noctilucent clouds

PSC - Polar stratospheric clouds

REP - relativistic electron precipitation

SA - solar activity

SCR - solar cosmic ray

SEP - solar energetic partcile

SPE - solar proton event

(SEPs event $\equiv \mathrm{SPE} \equiv \mathrm{SCRs}$ ) 


\section{Author contributions}

All co-authors contributed the Introduction and Summary.

IU, GB, ER, ET and IM performed the Section 2.

ER, FA, AK, IM, GH, KA and KAN performed the Section 3.

\section{Acknowledgements}

The development of this article has resulted from work carried out by an international team of the International Space Science Institute (ISSI), Bern, Switzerland, on "Study of Cosmic Ray Influence upon Atmospheric Processes" and the fruitful discussion of this article was continued with an international team of the ISSI on "Specification of Ionization Sources Affecting Atmospheric Processes". The collaboration in the frame of COST Action ES1005 TOSCA is grateful acknowledgements. KAN acknowledges the support of an Early Career Fellowship from the Leverhulme Trust. IU acknowledges ReSoLVE Centre of Excellence (Academy of Finland Project No. 272157). KA, GH and KAN are grateful to Dr F.Marcz of the Hungarian Institute of Sciences provides the PG data for Fig. 42. The solar flux data used in Fig. 45 were provided by NOAA's National Geophysical Data Center.

The authors thank the Editor for assistance and are grateful to anonymous Reviewers for their efforts and valuable comments that helped us to improve the manuscript. A special thank is due to one Reviewer helped us a lot with many rounds of the manuscript processing, that is particularly valuable for us. 


\section{References}

J.A. Abreu, J. Beer, F. Steinhilber, S.M. Tobias, N.O. Weiss, For how long will the current grand maximum of solar activity persist? Geophys. Res. Lett. 352, L20109 (2008). doi:10.1029/2008GL035442

O. Adriani, G.C. Barbarino, G.A. Bazilevskaya, R. Bellotti, M. Boezio, E.A. Bogomolov, M. Bongi, V. Bonvicini, S. Borisov, S. Bottai, A. Bruno, F. Cafagna, D. Campana, R. Carbone, P. Carlson, M. Casolino, G. Castellini, M.P. De Pascale, C. De Santis, N. De Simone, V. Di Felice, V. Formato, A.M. Galper, L. Grishantseva, A.V. Karelin, S.V. Koldashov, S. Koldobskiy, S.Y. Krutkov, A.N. Kvashnin, A. Leonov, V. Malakhov, L. Marcelli, A.G. Mayorov, W. Menn, V.V. Mikhailov, E. Mocchiutti, A. Monaco, N. Mori, N. Nikonov, G. Osteria, F. Palma, P. Papini, M. Pearce, P. Picozza, C. Pizzolotto, M. Ricci, S.B. Ricciarini, L. Rossetto, R. Sarkar, M. Simon, R. Sparvoli, P. Spillantini, Y.I. Stozhkov, A. Vacchi, E. Vannuccini, G. Vasilyev, S.A. Voronov, Y.T. Yurkin, J. Wu, G. Zampa, N. Zampa, V.G. Zverev, M.S. Potgieter, E.E. Vos, Time Dependence of the Proton Flux Measured by PAMELA during the 2006 July-2009 December Solar Minimum. Astrophys. J. 765, 91 (2013). doi:10.1088/0004-637X/765/2/91

M. Aguilar, G. Alberti, B. Alpat, A. Alvino, G. Ambrosi, K. Andeen, H. Anderhub, L. Arruda, P. Azzarello, A. Bachlechner, et al., First Result from the Alpha Magnetic Spectrometer on the International Space Station: Precision Measurement of the Positron Fraction in Primary Cosmic Rays of 0.5-350 GeV. Phys. Rev. Lett. 110(14), 141102 (2013). doi:10.1103/PhysRevLett.110.141102

H.S. Ahluwalia, Timelines of cosmic ray intensity, Ap, IMF, and sunspot numbers since 1937. J. Geophys. Res. 116, 12106 (2011). doi:10.1029/2011JA017021

H.S. Ahluwalia, R.C. Ygbuhay, Testing baseline stability of some neutron monitors in Europe, Africa, and Asia. Adv. Space Res. 51, 1990-1995 (2013). doi:10.1016/j.asr.2013.01.014

A.C. Aikin, Energetic particle-induced enhancements of stratospheric nitric acid. Geophys. Res. Lett. 21, 859-862 (1994). doi:10.1029/94GL00914

A.C. Aikin, Production of stratospheric $\mathrm{HNO}_{3}$ by different ion-molecule reaction mechanisms. J. Geophys. Res. 102, 12921-12926 (1997). doi:10.1029/97JD00419

S.-I. Akasofu, The auroral oval, the auroral substorm, and their relations with the internal structure of the magnetosphere. Planet. Space Sci. 14, 587-595 (1966). doi:10.1016/00320633(66)90043-2

M.V. Alania, R. Modzelewska, A. Wawrzynczak, On the Relationship of the 27-day Variations of the Solar Wind Velocity and Galactic Cosmic Ray Intensity in Minimum Epoch of Solar Activity. Solar Phys. 270, 629-641 (2011). doi:10.1007/s11207-011-9778-6

K. Alanko, I.G. Usoskin, K. Mursula, G.A. Kovaltsov, Heliospheric modulation strength: Effective neutron monitor energy. Adv. Space Res. 32, 615-620 (2003). doi:10.1016/S02731177(03)00348-X

S.Y. Aleksandrin, A.M. Galper, L.A. Grishantzeva, S.V. Koldashov, L.V. Maslennikov, A.M. Murashov, P. Picozza, V. Sgrigna, S.A. Voronov, High-energy charged particle bursts in the near-Earth space as earthquake precursors. Ann. Geophys. 21, 597-602 (2003). doi:10.5194/angeo-21-597-2003

H.R. Anderson, Cosmic Ray Total Ionization, 1970-1972. J. Geophys. Res. 78, 3958-3960 (1973). doi:10.1029/JA078i019p03958

M.E. Andersson, P.T. Verronen, S. Wang, C.J. Rodger, M.A. Clilverd, B.R. Carson, Precipitating radiation belt electrons and enhancements of mesospheric hydroxyl during 2004-2009. J. Geophys. Res. 117, D09304 (2012). doi:10.1029/2011JD017246

M.E. Andersson, P.T. Verronen, C.J. Rodger, M.A. Clilverd, S. Wang, Longitudinal hotspots in the mesospheric $\mathrm{OH}$ variations due to energetic electron precipitation. Atmos. Chem. Phys. 14, 1095-1105 (2014a). doi:10.5194/acp-14-1095-2014

M.E. Andersson, P.T. Verronen, C.J. Rodger, M.A. Clilverd, A. Seppälä, Missing driver in the Sun-Earth connection from energetic electron precipitation impacts mesospheric ozone. Nature Communications 5, 5197 (2014b). doi:10.1038/ncomms6197

E. Arijs, G. Brasseur, Acetonitrile in the stratosphere and implications for positive ion composition. J. Geophys. Res. 91, 4003-4016 (1986). doi:10.1029/JD091iD03p04003

F. Arnold, Ion-induced nucleation of atmospheric water vapor at the mesopause. Planet Space Sci. 28, 1003-1009 (1980a). doi:10.1016/0032-0633(80)90061-6

F. Arnold, Multi-ion complexes in the stratosphere - Implications for trace gases and aerosol. Nature 284, 610 (1980b). doi:10.1038/284610a0 
F. Arnold, Solvated electrons in the upper atmosphere. Nature 294, 732 (1981). doi:10.1038/294732a0

F. Arnold, Ion nucleation - A potential source for statospheric aerosols. Nature 299, 134-137 (1982). doi:10.1038/299134a0

F. Arnold, Atmospheric Ions and Aerosol Formation. Space Sci. Rev. 137, 225-239 (2008). doi:10.1007/s11214-008-9390-8

F. Arnold, T. Buehrke, New H2SO4 and HSO3 vapour measurements in the stratosphere Evidence for a volcanic influence. Nature 301, 293-295 (1983). doi:10.1038/301293a0

F. Arnold, D. Krankowsky, Ion Composition and Electron- and Ion-loss Processes in the Earth's Atmosphere., in Dynamical and Chemical Coupling between the Neutral and Ionized Atmosphere, ed. by B. Grandal, A. Holtet, 1977, pp. 93-127

F. Arnold, T. Buehrke, S. Qiu, Evidence for stratospheric ozone-depleting heterogeneous chemistry on volcanic aerosols from El Chichon. Nature 348, 49 (1990). doi:10.1038/348049a0

F. Arnold, A.A. Viggiano, H. Schlager, Implications for trace gases and aerosols of large negative ion clusters in the stratosphere. Nature 297, 371-376 (1982). doi:10.1038/297371a0

F. Arnold, A. Kiendler, V. Wiedemer, S. Aberle, T. Stilp, R. Busen, Chemiion concentration measurements in jet engine exhaust at the ground: Implications for ion chemistry and aerosol formation in the wake of a jet aircraft. Geophys. Res. Lett. 27, 1723-1726 (2000). doi:10.1029/1999GL011096

J. Austin, K. Tourpali, E. Rozanov, H. Akiyoshi, S. Bekki, G. Bodeker, C. Brühl, N. Butchart, M. Chipperfield, M. Deushi, V.I. Fomichev, M.A. Giorgetta, L. Gray, K. Kodera, F. Lott, E. Manzini, D. Marsh, K. Matthes, T. Nagashima, K. Shibata, R.S. Stolarski, H. Struthers, W. Tian, Coupled chemistry climate model simulations of the solar cycle in ozone and temperature. J. Geophys. Res. 113, D11306 (2008). doi:10.1029/2007JD009391

D.N. Baker, T.I. Pulkkinen, Solar Disturbances and Correlated Geospace Responses: Relativistic Magnetospheric Electron Acceleration, in Correlated Phenomena at the Sun, in the Heliosphere and in Geospace, ed. by A. Wilson ESA Special Publication, vol. 415, 1997, p. 199

D.N. Baker, G.M. Mason, J.E. Mazur, A small spacecraft mission with large accomplishments. EOS Trans. 93, 325-326 (2012). doi:10.1029/2012EO340001

D.N. Baker, Effects of the Sun on the Earth's environment. J. Atmos. Sol.-Terr. Phys. 62, 1669-1681 (2000). doi:10.1016/S1364-6826(00)00119-X

D.N. Baker, R.A. Goldberg, F.A. Herrero, J.B. Blake, L.B. Callis, Satellite and rocket studies of relativistic electrons and their influence on the middle atmosphere. J. Atmos. Sol.-Terr. Phys. 55, 1619-1628 (1993). doi:10.1016/0021-9169(93)90167-W

D.N. Baker, J.B. Blake, L.B. Callis, J.R. Cummings, D. Hovestadt, S. Kanekal, B. Klecker, R.A. Mewaldt, R.D. Zwickl, Relativistic electron acceleration and decay time scales in the inner and outer radiation belts: SAMPEX. Geophys. Res. Lett. 21, (1994). doi:10.1029/93GL03532

D.N. Baker, A.N. Jaynes, X. Li, M.G. Henderson, S.G. Kanekal, G.D. Reeves, H.E. Spence, S.G. Claudepierre, J.F. Fennell, M.K. Hudson, R.M. Thorne, J.C. Foster, P.J. Erickson, D.M. Malaspina, J.R. Wygant, A. Boyd, C.A. Kletzing, A. Drozdov, Y.Y. Shprits, Gradual diffusion and punctuated phase space density enhancements of highly relativistic electrons: Van Allen Probes observations. Geophys. Res. Lett. 41, 1351-1358 (2014). doi:10.1002/2013GL058942

L. Barnard, M. Lockwood, M.A. Hapgood, M.J. Owens, C.J. Davis, F. Steinhilber, Predicting space climate change. Geophys. Res. Lett. 381, L16103 (2011). doi:10.1029/2011GL048489

C. Barth, K. Mankoff, S. Bailey, S. Solomon, Global observations of nitric oxide in the thermosphere. J. Geophys. Res. 108 (A1) (2003). doi:10.1029/2002JA009458

A.J.G. Baumgaertner, P. Jöckel, C. Brühl, Energetic particle precipitation in ECHAM5/MESSy1 - Part 1: Downward transport of upper atmospheric $\mathrm{NO}_{x}$ produced by low energy electrons. Atmos. Chem. Phys. 9, 2729-2740 (2009)

A.J.G. Baumgaertner, A. Seppälä, P. Jöckel, M.A. Clilverd, Geomagnetic activity related $\mathrm{NO}_{x}$ enhancements and polar surface air temperature variability in a chemistry climate model: modulation of the NAM index. Atmos. Chem. Phys. 11, 4521-4531 (2011). doi:10.5194/acp-11-4521-2011

G. Bazilevskaya, A. Svirzhevskaya, Solar Cosmic Rays In The Earth's Atmosphere As Measured In The Long-term Lpi Balloon Experiment, in EGS General Assembly Conference Abstracts. EGS General Assembly Conference Abstracts, vol. 27, 2002, p. 2827

G.A. Bazilevskaya, Solar cosmic rays in the near Earth space and the atmosphere. Adv. Space 
Res. 35, 458-464 (2005). doi:10.1016/j.asr.2004.11.019

G.A. Bazilevskaya, A.I. Sladkova, On Certain Features of the Energy Spectrum of Solar Events with Relativistic Protons. Izv. Akad. Nauk, Ser. Fiz. 67, 1431-1434 (2003)

G.A. Bazilevskaya, A.K. Svirzhevskaya, On the stratospheric measurements of cosmic rays. Space Sci. Rev. 85, 431-521 (1998)

G.A. Bazilevskaya, M.B. Krainev, V.S. Makhmutov, Effects of cosmic rays on the Earth's environment. J. Atmos. Solar-Terr. Phys. 62, 1577-1586 (2000). doi:10.1016/S13646826(00)00113-9

G.A. Bazilevskaya, I.G. Usoskin, E.O. Flückiger, R.G. Harrison, L. Desorgher, R. Bütikofer, M.B. Krainev, V.S. Makhmutov, Y.I. Stozhkov, A.K. Svirzhevskaya, N.S. Svirzhevsky, G.A. Kovaltsov, Cosmic ray induced ion production in the atmosphere. Space Sci. Rev. 137, 149-173 (2008). doi:10.1007/s11214-008-9339-y

G.A. Bazilevskaya, V.S. Makhmutov, Y.I. Stozhkov, A.K. Svirzhevskaya, N.S. Svirzhevsky, Solar proton events recorded in the stratosphere during cosmic ray balloon observations in 1957-2008. Adv. Space Res. 45, 603-613 (2010). doi:10.1016/j.asr.2009.11.009

K.V. Beard, H.T. Ochs, C.H. Twohy, Aircraft measurements of high average charges on cloud drops in layer clouds. Geophys. Res. Lett. 31, L14111 (2004). doi:10.1029/2004GL020465

A.V. Belov, Forbush Effects and Their Connection with Solar, Interplanetary and Geomagnetic Phenomena, Proc IAU Symp. 257, 439-450 (2009). doi:10.1017/S1743921309029676

J.B. Blake, M.D. Looper, D.N. Baker, R. Nakamura, B. Klecker, D. Hovestadt, New high temporal and spatial resolution measurements by SAMPEX of the precipitation of relativistic electrons. Adv. Space Res. 18, 171-186 (1996)

C.B. Boyle, P.H. Reiff, M.R. Hairston, Empirical polar cap potentials. J. Geophys. Res. 102, 111-126 (1997). doi:10.1029/96JA01742

G.P. Brasseur, S. Solomon, Aeronomy of the Middle Atmosphere: Chemistry and Physics of the Stratosphere and Mesosphere 2005

D.H. Brautigam, M.S. Gussenhoven, D.A. Hardy, A statistical study on the effects of IMF $\mathrm{B}(\mathrm{z})$ and solar wind speed on auroral ion and electron precipitation. J. Geophys. Res. 96, 5525-5538 (1991). doi:10.1029/91JA00157

R. Bučík, K. Kudela, S.N. Kuznetsov, Satellite observations of lightning-induced hard Xray flux enhancements in the conjugate region. Ann. Geophys. 24, 1969-1976 (2006). doi:10.5194/angeo-24-1969-2006

M. Calisto, I. Usoskin, E. Rozanov, T. Peter, Influence of Galactic Cosmic Rays on atmospheric composition and dynamics. Atmos. Chem. Phys. 11, 4547-4556 (2011). doi:10.5194/acp11-4547-2011

M. Calisto, P.T. Verronen, E. Rozanov, T. Peter, Influence of a carrington-like event on the atmospheric chemistry, temperature and dynamics. Atmos. Chem. Phys. 12, 8679-8686 (2012). doi:10.5194/acp-12-8679-2012.

L.B. Callis, M. Natarajan, J.D. Lambeth, Observed and calculated mesospheric NO, 1992-1997. Geophys. Res. Lett. 29 (2), D1030 (2002). doi:10.1029/2001GL013995

L.B. Callis, R.E. Boughner, D.N. Baker, J.B. Blake, J.D. Lambeth, Precipitating relativistic electrons - Their long-term effect on stratospheric odd nitrogen levels. J. Geophys. Res. 96, 2939-2976 (1991). doi:10.1029/90JD02184

L.B. Callis, R.E. Boughner, D.N. Baker, R.A. Mewaldt, J. Bernard Blake, R.S. Selesnick, J.R. Cummings, M. Natarajan, G.M. Mason, J.E. Mazur, Precipitating electrons: Evidence for effects on mesospheric odd nitrogen. Geophys. Res. Lett. 23, 1901-1904 (1996). doi:10.1029/96GL01787

L.B. Callis, M. Natarajan, J.D. Lambeth, D.N. Baker, Solar atmospheric coupling by electrons (SOLACE) 2. Calculated stratospheric effects of precipitating electrons, 1979-1988. J. Geophys. Res. 103, 28421-28438 (1998). doi:10.1029/98JD02407

J. Calogovic, C. Albert, F. Arnold, J. Beer, L. Desorgher, E.O. Flueckiger, Sudden cosmic ray decreases: No change of global cloud cover. Geophys. Res. Lett. 370, 03802 (2010). doi:10.1029/2009GL041327

H.V. Cane, Coronal Mass Ejections and Forbush Decreases. Space Sci. Rev. 93, 55-77 (2000). doi:10.1023/A:1026532125747

K.S. Carslaw, R.G. Harrison, J. Kirkby, Cosmic Rays, Clouds, and Climate. Science 298, 1732-1737 (2002). doi:10.1126/science.1076964

A.N. Charakhchyan, Reviews of topical problems: Investigation of stratosphere cosmic ray intensity fluctuations induced by processes on the Sun. Soviet Physics Uspekhi 7, 358-374 (1964) 
S.P. Christon, D.J. Williams, D.G. Mitchell, C.Y. Huang, L.A. Frank, Spectral characteristics of plasma sheet ion and electron populations during disturbed geomagnetic conditions. J. Geophys. Res. 96, 1-22 (1991). doi:10.1029/90JA01633

T.G. Chronis, Investigating Possible Links between Incoming Cosmic Ray Fluxes and Lightning Activity over the United States. J. Clim. 22, 5748 (2009). doi:10.1175/2009JCLI2912.1

V.P. Chuprova, S.K. Gerasimova, V.G. Grigoryev, P.A. Krivoshapkin, G.F. Krymsky, V.P. Mamrukova, V.M. Migunov, A.N. Prikhodko, G.V. Shafer, G.V. Skripin, Y.Y. Sorokin, S.A. Starodubtsev, V.E. Timofeev, The brief history of experimental research of cosmic ray variations in Yakutia. Adv. Space Res. 44, 1200-1206 (2009). doi:10.1016/j.asr.2008.12.024

M.A. Clilverd, C.J. Rodger, R.M. Millan, J.G. Sample, M. Kokorowski, M.P. McCarthy, T. Ulich, T. Raita, A.J. Kavanagh, E. Spanswick, Energetic particle precipitation into the middle atmosphere triggered by a coronal mass ejection. J. Geophys. Res. 112, A12206 (2007). doi:10.1029/2007JA012395

M.A. Clilverd, C.J. Rodger, T. Moffat-Griffin, E. Spanswick, P. Breen, F.W. Menk, R.S. Grew, K. Hayashi, I.R. Mann, Energetic outer radiation belt electron precipitation during recurrent solar activity. J. Geophys. Res. 115, A8323 (2010a). doi:10.1029/2009JA015204

M.A. Clilverd, C.J. Rodger, R.J. Gamble, T. Ulich, T. Raita, A. SeppäLä, J.C. Green, N.R. Thomson, J.-A. Sauvaud, M. Parrot, Ground-based estimates of outer radiation belt energetic electron precipitation fluxes into the atmosphere.J. Geophys. Res. 115, A12304 (2010b). doi:10.1029/2010JA015638

W.E. Cobb, Evidence of a solar influence on the atmospheric electric elements at mauna loa observatory. Monthly Weather Review 95, 905 (1967)

W.E. Cobb, Balloon Measurements of the Air-earth Current Density at the South Pole Before and After a Solar Flare, in Conference on Cloud Physics and Atmospheric Electricity, 1978, pp. 552-554

D.J. Cooke, J.E. Humble, M.A. Shea, D.F. Smart, N. Lund, I.L. Rasmussen, B. Byrnak, P. Goret, N. Petrou, On cosmic-ray cut-off terminology. Nuovo Cimento C 14, 213-234 (1991)

P.J. Crutzen, Photochemical reactions initiated by and influencing ozone in the troposphere. Tellus 26, 47 (1974)

P.J. Crutzen, I.S.A. Isaksen, G.C. Reid, Solar proton events - Stratospheric sources of nitric oxide. Science 189, 457-459 (1975). doi:10.1126/science.189.4201.457

A.C. Cummings, E.C. Stone, Anomalous Cosmic Rays and Solar Modulation. Space Sci. Rev. 83, 51-62 (1998)

J. Curtius, R. Weigel, H.-J. Vössing, H. Wernli, A. Werner, C.-M. Volk, P. Konopka, M. Krebsbach, C. Schiller, A. Roiger, H. Schlager, V. Dreiling, S. Borrmann, Observations of meteoric material and implications for aerosol nucleation in the winter Arctic lower stratosphere derived from in situ particle measurements. Atmos. Chem. Phys. 5, 30533069 (2005)

P. Dalin, N. Pertsev, V. Romejko, Notes on historical aspects on the earliest known observations of noctilucent clouds. History of Geo- and Space Sciences 3, 87-97 (2012). doi:10.5194/hgss3-87-2012

D.W. Datlowe, W.L. Imhof, Cyclotron resonance precipitation of energetic electrons from the inner magnetosphere. J. Geophys. Res. 95, 6477-6491 (1990). doi:10.1029/JA095iA05p06477

M.T. DeLand, E.P. Shettle, G.E. Thomas, J.J. Olivero, Solar backscattered ultraviolet (SBUV) observations of polar mesospheric clouds (PMCs) over two solar cycles. J. Geophys. Res. 108, D8445 (2003). doi:10.1029/2002JD002398

L. Desorgher, E.O. Flückiger, M. Gurtner, M.R. Moser, R. Bütikofer, Atmocosmics: a Geant 4 Code for Computing the Interaction of Cosmic Rays with the Earth's Atmosphere. Intern. J. Modern Phys. A 20, 6802-6804 (2005). doi:10.1142/S0217751X05030132

L.I. Dorman, Cosmic Rays in the Earth's Atmosphere and Underground (Kluwer Academic Publishers, Dordrecht, Netherlands, 2004)

R.H. Eather, The auroral oval. - A reevaluation. Rev. Geophys. Space Phys. 11, 155-167 (1973). doi:10.1029/RG011i001p00155

T. Egorova, E. Rozanov, Y. Ozolin, A. Shapiro, M. Calisto, T. Peter, W. Schmutz, The atmospheric effects of October 2003 solar proton event simulated with the chemistry-climate model SOCOL using complete and parameterized ion chemistry. J. Atmos. Solar-Terr. Phys. 73, 356-365 (2011). doi:10.1016/j.jastp.2010.01.009

S. Eichkorn, S. Wilhelm, H. Aufmhoff, K.H. Wohlfrom, F. Arnold, Cosmic ray-induced aerosol-formation: First observational evidence from aircraft-based ion mass spectrome- 
ter measurements in the upper troposphere. Geophys. Res. Lett. 29(14), D1698 (2002). doi:10.1029/2002GL015044

F.L. Eisele, Natural and transmission line produced positive ions. J. Geophys. Res. 94, 63096318 (1989). doi:10.1029/JD094iD05p06309

B.A. Emery, V. Coumans, D.S. Evans, G.A. Germany, M.S. Greer, E. Holeman, K. KadinskyCade, F.J. Rich, W. Xu, Seasonal, Kp, solar wind, and solar flux variations in long-term singlepass satellite estimates of electron and ion auroral hemispheric power. J. Geophys. Res. 113, A6311 (2008). doi:10.1029/2007JA012866

M.B. Enghoff, H. Svensmark, The role of atmospheric ions in aerosol nucleation a review. Atmos. Chem. Phys. 8, 4911-4923 (2008)

M.B. Enghoff, J.O.P. Pedersen, U.I. Uggerhøj, S.M. Paling, H. Svensmark, Aerosol nucleation induced by a high energy particle beam. Geophys. Res. Lett. 38, L09805 (2011). doi:10.1029/2011GL047036

V. Eyring, J.-F. Lamarque, P. Hess, F. Arfeuille, K. Bowman, M. Chipperfield, B. Duncan, A. Fiore, A. Gettelman, M. Giorgetta, C. Granier, M. Hegglin1, D. Kinnison, M. Kunze1, U. Langematz, B.-P. Luo, M. Randall, K. Matthes, P. Newman, T. Peter, A. Robock, T. Ryerson, A. Saiz-Lopez, R. Salawitch, M. Schultz, T. Shepherd, D. Shindell, J. Staehelin, S. Tegtmeier, L. Thomason, S. Tilmes, J.-P. Vernier, D. Waugh, Y. P., Overview of IGAC/SPARC Chemistry-Climate Model Initiative (CCMI) Community Simulations in support of upcoming Ozone and Climate Assessments. SPARC newsletter, 48-66 (2013)

P. Fabian, J.A. Pyle, R.J. Wells, The August 1972 solar proton event and the atmospheric ozone layer. Nature 277, 458-460 (1979). doi:10.1038/277458a0

W.M. Farrell, M.D. Desch, Solar proton events and the fair weather electric field at ground. Geophys. Res. Lett. 29(9), 1323 (2002). doi:10.1029/2001GL013908

I.I. Feldshtein, I.I. Galperin, An alternative interpretation of auroral precipitation and luminosity observations from the DE, DMSP, AUREOL, and Viking satellites in terms of their mapping to the nightside magnetosphere. J. Atmos. Terr. Phys. 55, 105-121 (1993)

Y.I. Feldstein, Auroral oval. J. Geophys. Res. 78, 1210 (1973). doi:10.1029/JA078i007p01210

E.E. Ferguson, F. Arnold, Ion Chemistry of the Stratosphere. Account of Chem. Res. 14, 327-334 (1981)

E.E. Ferguson, F.C. Fehsenfeld, D.L. Albritton, Chapter 2 - Ion Chemistry of the Earth's Atmosphere, in Gas Phase Ion Chemistry, ed. by M.T. BOWERS (Academic Press, New York, 1979), pp. 45-82. ISBN 978-0-12-120801-1. doi:http://dx.doi.org/10.1016/B978-0-12-120801-1.50008-9. http://www.sciencedirect.com/science/article/pii/B9780121208011500089

J.E. Foat, R.P. Lin, D.M. Smith, F. Fenrich, R. Millan, I. Roth, K.R. Lorentzen, M.P. McCarthy, G.K. Parks, J.P. Treilhou, First detection of a terrestrial MeV X-ray burst. J. Geophys. Res. 25, 4109-4112 (1998). doi:10.1029/1998GL900134

B. Fogle, B. Haurwitz, Noctilucent Clouds. Space Sci. Rev. 6, 279-340 (1966). doi:10.1007/BF00173768

S.E. Forbush, World-Wide Cosmic-Ray Variations, 1937-1952. J. Geophys. Res. 59, 525-542 (1954)

S.E. Forbush, Cosmic-Ray Intensity Variations during Two Solar Cycles. J. Geophys. Res. 63, 651-669 (1958). doi:10.1029/JZ063i004p00651

P.M. Forster, V.I. Fomichev, E. Rozanov, C. Cagnazzo, A.I. Jonsson, U. Langematz, B. Fomin, M.J. Iacono, B. Mayer, E. Mlawer, G. Myhre, R.W. Portmann, H. Akiyoshi, V. Falaleeva, N. Gillett, A. Karpechko, J. Li, P. Lemennais, O. Morgenstern, S. Oberländer, M. Sigmond, K. Shibata, Evaluation of radiation scheme performance within chemistry climate models. J. Geophys. Res. 116, D10302 (2011). doi:10.1029/2010JD015361

H.U. Frey, Localized aurora beyond the auroral oval. Reviews of Geophysics 45, 1003 (2007). doi:10.1029/2005RG000174

F. Friederich, M. Sinnhuber, B. Funke, T. von Clarmann, J. Orphal, Local impact of solar variation on $\mathrm{NO}_{2}$ in the lower mesosphere and upper stratosphere from 2007 to 2012. Atmos. Chem. Phys. 14, 4055-4064 (2014). doi:10.5194/acp-14-4055-2014

C. Fröhlich, J. Lean, The Sun's total irradiance: Cycles, trends and related climate change uncertainties since 1976. Geophys. Res. Lett. 25, 4377-4380 (1998). doi:10.1029/1998GL900157

B. Funke, M. López-Puertas, S. Gil-López, T. von Clarmann, G.P. Stiller, H. Fischer, S. Kellmann, Downward transport of upper atmospheric $\mathrm{NO}_{x}$ into the polar stratosphere and lower mesosphere during the Antarctic 2003 and Arctic 2002/2003 winters. J. Geophys. 
Res. 110, D24308 (2005). doi:10.1029/2005JD006463

B. Funke, A. Baumgaertner, M. Calisto, T. Egorova, C.H. Jackman, J. Kieser, A. Krivolutsky, M. López-Puertas, D.R. Marsh, T. Reddmann, E. Rozanov, S.-M. Salmi, M. Sinnhuber, G.P. Stiller, P.T. Verronen, S. Versick, T. von Clarmann, T.Y. Vyushkova, N. Wieters, J.M. Wissing, Composition changes after the Halloween solar proton event: the High Energy Particle Precipitation in the Atmosphere (HEPPA) model versus MIPAS data intercomparison study. Atmos. Chem. Phys. 11, 9089-9139 (2011). doi:10.5194/acp-11-9089-2011

B. Funke, M. López-Puertas, G.P. Stiller, T. Clarmann, Mesospheric and stratospheric $\mathrm{NO}_{y}$ produced by energetic particle precipitation during 2002-2012. J. Geophys. Res. 119, 44294446 (2014). doi:10.1002/2013JD021404

T. Gaisser, Cosmic Rays and Particle Physics 1990

A.M. Galper, S.V. Koldashov, S.A. Voronov, High energy particle flux variations as earthquake predictors. Adv. Space Res. 15, 131-134 (1995). doi:10.1016/0273-1177(95)00085-S

G.A. Germany, G.K. Parks, H. Ranganath, R. Elsen, P.G. Richards, W. Swift, J.F. Spann, M. Brittnacher, Analysis of auroral morphology: Substorm precursor and onset on January 10, 1997. J. Geophys. Res. 25, 3043-3046 (1998). doi:10.1029/98GL01220

S.E. Gibson, G. de Toma, B. Emery, P. Riley, L. Zhao, Y. Elsworth, R.J. Leamon, J. Lei, S. McIntosh, R.A. Mewaldt, B.J. Thompson, D. Webb, The Whole Heliosphere Interval in the Context of a Long and Structured Solar Minimum: An Overview from Sun to Earth. Solar Phys. 274, 5-27 (2011). doi:10.1007/s11207-011-9921-4

A. Gil, K. Iskra, R. Modzelewska, M.V. Alania, On the 27-day variations of the galactic cosmic ray anisotropy and intensity for different periods of solar magnetic cycle. Adv. Space. Res. 35, 687-690 (2005). doi:10.1016/j.asr.2005.03.018

O.H. Gish, Evaluation and interpretation of the columnar resistance of the atmosphere. Terr. Magn. Atmos. Electr. 49, 159-168 (1944)

L.J. Gray, J. Beer, M. Geller, J.D. Haigh, M. Lockwood, K. Matthes, U. Cubasch, D. Fleitmann, G. Harrison, L. Hood, J. Luterbacher, G.A. Meehl, D. Shindell, B. van Geel, W. White, Solar influence on climate. Rev. Geophys. 48, 4001 (2010). doi:10.1029/2009RG000282

P.K.F. Grieder, Cosmic Rays at Earth (Elsevier Science, Amsterdam, 2001)

W. Gringel, Examinations of Electrical Conductivity of Air Taking Into Account Solar Activity and the Aerosol Particle Concentration up to Heights of $35 \mathrm{Km}$, PhD thesis, Tuebingen, Universitaet, Fachbereich Physik, Doktor der Naturwissenschaften Dissertation, 1978. 108 p. In German., 1978

A.V. Gurevich, K.P. Zybin, Runaway Breakdown and the Mysteries of Lightning. Physics Today 58(5), 050000-43 (2005). doi:10.1063/1.1995746

D.A. Hardy, M.S. Gussenhoven, D. Brautigam, A statistical model of auroral ion precipitation. J. Geophys. Res. 94, 370-392 (1989). doi:10.1029/JA094iA01p00370

D.A. Hardy, M.S. Gussenhoven, E. Holeman, A statistical model of auroral electron precipitation. J. Geophys. Res. 90, 4229-4248 (1985). doi:10.1029/JA090iA05p04229

D.A. Hardy, M.S. Gussenhoven, R. Raistrick, W.J. McNeil, Statistical and functional representations of the pattern of auroral energy flux, number flux, and conductivity. J. Geophys. Res. 92, 12275-12294 (1987). doi:10.1029/JA092iA11p12275

D.A. Hardy, M.S. Gussenhoven, D. Brautigam, W. McNeil, A statistical model of auroral ion precipitation. II - Functional representation of the average patterns. J. Geophys. Res. 96, 5539-5547 (1991). doi:10.1029/90JA02451

R.G. Harrison, Cloud Formation and the Possible Significance of Charge for Atmospheric Condensation and Ice Nuclei. Space Sci.Rev. 94, 381-396 (2000)

R.G. Harrison, The Carnegie Curve. Surv. Geophys. 34, 209-232 (2013). doi:10.1007/s10712012-9210-2

R.G. Harrison, M.H.P. Ambaum, Observed atmospheric electricity effect on clouds. Envir. Res. Lett. 4(1), 014003 (2009). doi:10.1088/1748-9326/4/1/014003

R.G. Harrison, M.H.P. Ambaum, Observing Forbush decreases in cloud atShetland. J. Atmos. Solar-Terr. Phys. 72, 1408-1414 (2010). doi:10.1016/j.jastp.2010.09.025

R.G. Harrison, K.S. Carslaw, Ion-aerosol-cloud processes in the lower atmosphere. Rev. Geophys. 41, 1012 (2003). doi:10.1029/2002RG000114

R.G. Harrison, H. Tammet, Ions in the Terrestrial Atmosphere and Other Solar System Atmospheres. Space Sci. Rev. 137, 107-118 (2008). doi:10.1007/s11214-008-9356-x

R.G. Harrison, I. Usoskin, Solar modulation in surface atmospheric electricity. J. Atmos. SolarTerr. Phys. 72, 176-182 (2010). doi:10.1016/j.jastp.2009.11.006 
C.J. Hatton, The Neutron Monitor, in Processes in Elementary Particle and Cosmic Ray Physics, ed. by Wilson, J.G. and Wouthuysen, S.A (North-Holland Publ. Comp., Amsterdam-London, 1971), pp. 3-100

M.G. Heaps, Parametrization of the cosmic ray ion-pair production rate above $18 \mathrm{~km}$. Planet. Space Sci. 26, 513-517 (1978). doi:10.1016/0032-0633(78)90041-7

D.F. Heath, A.J. Krueger, P.J. Crutzen, Solar proton event - Influence on stratospheric ozone. Science 197, 886-889 (1977). doi:10.1126/science.197.4306.886

G. Henschen, F. Arnold, New positive ion species in the stratosphere. Nature 291, 211-213 (1981). doi:10.1038/291211a0

A. Hirsikko, T. Nieminen, S. Gagné, K. Lehtipalo, H.E. Manninen, M. Ehn, U. Hõrrak, V.M. Kerminen, L. Laakso, P.H. McMurry, A. Mirme, S. Mirme, T. Petäjä, H. Tammet, V. Vakkari, M. Vana, M. Kulmala, Atmospheric ions and nucleation: a review of observations. Atm. Chem. Phys. 11, 767-798 (2011). doi:10.5194/acp-11-767-2011

R.H. Holzworth, F.S. Mozer, Direct evidence of solar flare modification of stratospheric electric fields. J. Geophys. Res. 84, 363-367 (1979). doi:10.1029/JC084iC01p00363

R.H. Holzworth, K.W. Norville, P.R. Williamson, Solar flare perturbations in stratospheric current systems. Geophys. Res. Lett. 14, 852-855 (1987). doi:10.1029/GL014i008p00852

W.A. Hoppel, A.R. V., J.C. Willett, Atmospheric Electricity in the Planetary Boundary Layer. (The National Academies Press, Washington, 1986). ISBN 9780309036801

R.B. Horne, R.M. Thorne, Relativistic electron acceleration and precipitation during resonant interactions with whistler-mode chorus. Geophys. Res. Lett. 30, L1527 (2003). doi:10.1029/2003GL016973

R.B. Horne, M.M. Lam, J.C. Green, Energetic electron precipitation from the outer radiation belt during geomagnetic storms. Geophys. Res. Lett. 36, L19104 (2009). doi:10.1029/2009GL040236

H. Hu, R.H. Holzworth, Observations and parameterization of the stratospheric electrical conductivity. J. Geophys. Res. 101, 29539-29552 (1996). doi:10.1029/96JD01060

T. Iijima, T.A. Potemra, Field-aligned currents in the dayside cusp observed by Triad. J. Geophys. Res. 81, 5971-5979 (1976a). doi:10.1029/JA081i034p05971

T. Iijima, T.A. Potemra, The amplitude distribution of field-aligned currents at northern high latitudes observed by Triad. J. Geophys. Res. 81, 2165-2174 (1976b). doi:10.1029/JA081i013p02165

W.L. Imhof, H.D. Voss, J. Mobilia, D.W. Datlowe, E.E. Gaines, The precipitation of relativistic electrons near the trapping boundary. J. Geophys. Res. 96, 5619-5629 (1991). doi:10.1029/90JA02343

IPCC, 2007: Climate Change 2007: The Scientific Basis. Contribution of Working Group I to the Fourth Assessment Report of the Intergovernmental Panel on Climate Change, edited by: Solomon, S., Qin, D., Manning, M., Chen, Z., Marquis, M., Averyt, K. B., Tignor, M., and Miller, H. L., Cambridge University Press, Cambridge, United Kingdom and New York, NY, USA, 996 (2007)

IPCC, 2013: Climate Change 2013: The Scientific Basis. Contribution of Working Group I to the Fifth Assessment Report of the Intergovernmental Panel on Climate Change, edited by: Stocker, T.F., D. Qin, G.-K. Plattner, M. Tignor, S.K. Allen, J. Boschung, A. Nauels, Y. Xia, V. Bex and P.M. Midgley, Cambridge University Press, Cambridge, United Kingdom and New York, NY, USA, 1029 (2013)

C.H. Jackman, J.E. Frederick, R.S. Stolarski, Production of odd nitrogen in the stratosphere and mesosphere - An intercomparison of source strengths. J. Geophys. Res. 85, 7495-7505 (1980). doi:10.1029/JC085iC12p07495

C.H. Jackman, M.C. Cerniglia, J.E. Nielsen, D.J. Allen, J.M. Zawodny, R.D. McPeters, A.R. Douglass, J.E. Rosenfield, R.B. Rood, Two-dimensional and three-dimensional model simulations, measurements, and interpretation of the influence of the October 1989 solar proton events on the middle atmosphere. J. Geophys. Res. 100, 11641-11660 (1995). doi:10.1029/95JD00369

C.H. Jackman, R.D. McPeters, G.J. Labow, E.L. Fleming, C.J. Praderas, J.M. Russell, Northern hemisphere atmospheric effects due to the July 2000 Solar Proton Event. Geophys. Res. Lett. 28, 2883-2886 (2001). doi:10.1029/2001GL013221

C.H. Jackman, M.T. Deland, G.J. Labow, E.L. Fleming, D.K. Weisenstein, M.K.W. Ko, M. Sinnhuber, J.M. Russell, Neutral atmospheric influences of the solar proton events in October-November 2003. J. Geophys. Res. 110, A09S27 (2005). doi:10.1029/2004JA010888 
C.H. Jackman, C.E. Randall, V.L. Harvey, S. Wang, E.L. Fleming, M. López-Puertas, B. Funke, P.F. Bernath, Middle atmospheric changes caused by the January and March 2012 solar proton events. Atmos. Chem. Phys. 14, 1025-1038 (2014). doi:10.5194/acp-14-10252014

C.H. Jackman, A.R. Douglass, R.B. Rood, R.D. McPeters, P.E. Meade, Effect of solar proton events on the middle atmosphere during the past two solar cycles as computed using a two-dimensional model. J. Geophys. Res. 95, 7417-7428 (1990)

J.F. Janni, Proton Range-Energy Tables, 1 keV-10 GeV, Energy Loss, Range, Path Length, Time-of-Flight, Straggling, Multiple Scattering, and Nuclear Interaction Probability. Part I. For 63 Compounds. Atom. Data Nuc. Data Tables 27, 147-339 (1982)

J.R. Jokipii, J. Kóta, Three-DImensional Cosmic-Ray Simulations: Heliographic Latitude and Current-Sheet Tilt. Space Sci. Rev. 72, 379-384 (1995). doi:10.1007/BF00768808

F.W. Jones, Comparison of two Solutions of a Geomagnetic Problem. Geophysical Journal International 39, 623-624 (1974). doi:10.1111/j.1365-246X.1974.tb05478.x

A.P. Jordan, H.E. Spence, J.B. Blake, D.N.A. Shaul, Revisiting two-step Forbush decreases. J. Geophys. Res. 116, 11103 (2011). doi:10.1029/2011JA016791

J. Kazil, R.G. Harrison, E.R. Lovejoy, Tropospheric New Particle Formation and the Role of Ions. Space Sci. Rev. 137, 241-255 (2008). doi:10.1007/s11214-008-9388-2

J. Kazil, E. Kopp, S. Chabrillat, J. Bishop, The University of Bern Atmospheric Ion Model: Time-dependent modeling of the ions in the mesosphere and lower thermosphere. J. Geophys. Res. 108, D4432 (2003). doi:10.1029/2002JD003024

J. Kazil, E.R. Lovejoy, M.C. Barth, K. O'Brien, Aerosol nucleation over oceans and the role of galactic cosmic rays. Atmosph. Chem. Phys. 6, 4905-4924 (2006)

J. Kazil, K. Zhang, P. Stier, J. Feichter, U. Lohmann, K. O'Brien, The present-day decadal solar cycle modulation of Earth's radiative forcing via charged $\mathrm{H}_{2} \mathrm{SO}_{4} / \mathrm{H}_{2} \mathrm{O}$ aerosol nucleation. Geophys. Res. Lett. 39, L2805 (2012). doi:10.1029/2011GL050058

J. Kirkby, J. Curtius, J. Almeida, E. Dunne, J. Duplissy, S. Ehrhart, A. Franchin, S. Gagne, L. Ickes, A. Kurten, A. Kupc, A. Metzger, F. Riccobono, L. Rondo, S. Schobesberger, G. Tsagkogeorgas, D. Wimmer, A. Amorim, F. Bianchi, M. Breitenlechner, A. David, J. Dommen, A. Downard, M. Ehn, R.C. Flagan, S. Haider, A. Hansel, D. Hauser, W. Jud, H. Junninen, F. Kreissl, A. Kvashin, A. Laaksonen, K. Lehtipalo, J. Lima, E.R. Lovejoy, V. Makhmutov, S. Mathot, J. Mikkila, P. Minginette, S. Mogo, T. Nieminen, A. Onnela, P. Pereira, T. Petaja, R. Schnitzhofer, J.H. Seinfeld, M. Sipila, Y. Stozhkov, F. Stratmann, A. Tome, J. Vanhanen, Y. Viisanen, A. Vrtala, P.E. Wagner, H. Walther, E. Weingartner, H. Wex, P.M. Winkler, K.S. Carslaw, D.R. Worsnop, U. Baltensperger, M. Kulmala, Role of sulphuric acid, ammonia and galactic cosmic rays in atmospheric aerosol nucleation. Nature 476(7361), 429-433 (2011). doi:10.1038/nature10343. http://dx.doi.org/10.1038/nature10343

S. Kirkwood, P. Dalin, A. Réchou, Noctilucent clouds observed from the UK and Denmark - trends and variations over 43 years. Ann. Geophys. 26, 1243-1254 (2008). doi:10.5194/angeo-26-1243-2008

N.G. Kleimenova, O.V. Kozyreva, S. Michnowski, M. Kubicki, Effect of magnetic storms in variations in the atmospheric electric field at midlatitudes. Geomag. Aeronom. 48, 622-630 (2008). doi:10.1134/S0016793208050071

C.A. Kletzing, J.D. Scudder, E.E. Dors, C. Curto, Auroral source region: Plasma properties of the high-latitude plasma sheet. J. Geophys. Res. 108, A1360 (2003). doi:10.1029/2002JA009678

N.N. Klimin, V.Y. Rivkind, V.A. Pachin, Collision efficiency calculation model as a software tool for microphysics of electrified clouds. Meteorol. Atmosph. Phys. 53, 111-120 (1994). doi:10.1007/BF01031908

M. Kokorowski, J.G. Sample, R.H. Holzworth, E.A. Bering, S.D. Bale, J.B. Blake, A.B. Collier, A.R.W. Hughes, E. Lay, R.P. Lin, M.P. McCarthy, R.M. Millan, H. Moraal, T.P. O'Brien, G.K. Parks, M. Pulupa, B.D. Reddell, D.M. Smith, P.H. Stoker, L. Woodger, Rapid fluctuations of stratospheric electric field following a solar energetic particle event. Geophys. Res. Lett. 33, L20105 (2006). doi:10.1029/2006GL027718

J.E. Kristjánsson, C.W. Stjern, F. Stordal, A.M. Fjæraa, G. Myhre, K. Jónasson, Cosmic rays, cloud condensation nuclei and clouds - a reassessment using MODIS data. Atmos. Chem. Phys. 8, 7373-7387 (2008)

A. Krivolutsky, A. Kuminov, T. Vyushkova, Ionization of the atmosphere caused by solar protons and its influence on ozonosphere of the Earth during 1994-2003. J. Atmos. Solar- 
Terrest. Phys. 67, 105-117 (2005). doi:10.1016/j.jastp.2004.08.004

A.A. Krivolutsky, Cosmic ray influence on chemical composition of the atmosphere of the earth. Adv. Space Res. 27, 1993-2002 (2001). doi:10.1016/S0273-1177(01)00296-4

A.A. Krivolutsky, A.I. Repnev, Impact of space energetic particles on the Earth's atmosphere (a review). Geomag. Aeronom. 52, 685-716 (2012). doi:10.1134/S0016793212060060

A.A. Krivolutsky, A.V. Klyuchnikova, G.R. Zakharov, T.Y. Vyushkova, A.A. Kuminov, Dynamical response of the middle atmosphere to solar proton event of July 2000: Three-dimensional model simulations. Adv. Space Res. 37, 1602-1613 (2006). doi:10.1016/j.asr.2005.05.115

A.A. Krivolutsky, A.A. Kuminov, A.A. Kukoleva, A.I. Repnev, N.K. Pereyaslova, M.N. Nazarova, Solar proton activity during cycle 23 and changes in the ozonosphere: Numerical simulation and analysis of observational data. Geomagn. Aeronom. 48, 432-445 (2008). doi:10.1134/S0016793208040038

L. Laakso, T. Anttila, K.E.J. Lehtinen, P.P. Aalto, M. Kulmala, U. Hõrrak, J. Paatero, M. Hanke, F. Arnold, Kinetic nucleation and ions in boreal forest particle formation events. Atmos. Chem. Phys. 4, 2353-2366 (2004)

B. Laken, D. Kniveton, A. Wolfendale, Forbush decreases, solar irradiance variations, and anomalous cloud changes. J. Geophys. Res. 116, D9201 (2011). doi:10.1029/2010JD014900

B.A. Laken, E. Pallé, J. Calogović, E.M. Dunne, A cosmic ray-climate link and cloud observations. J. Space Weather Space Clim. 2(26), 260000 (2012). doi:10.1051/swsc/2012018

D.J. Lary, Catalytic destruction of stratospheric ozone. J. Geophys. Res. 102, 21515 (1997). doi:10.1029/97JD00912

J. Laštovička, P. Križan, Geomagnetic storms, Forbush decreases of cosmic rays and total ozone at northern higher middle latitudes. J. Atmos. Solar-Terrest. Phys. 67, 119-124 (2005). doi:10.1016/j.jastp.2004.07.021

L.L. Lazutin, Solar Cosmic Rays Recorded in the Stratosphere in September 1963., in International Cosmic Ray Conference. International Cosmic Ray Conference, vol. 1, 1965, p. 271

S.-H. Lee, J.M. Reeves, J.C. Wilson, D.E. Hunton, A.A. Viggiano, T.M. Miller, J.O. Ballenthin, L.R. Lait, Particle Formation by Ion Nucleation in the Upper Troposphere and Lower Stratosphere. Science 301, 1886-1889 (2003). doi:10.1126/science.1087236

K. Liou, P.T. Newell, D.G. Sibeck, C.-I. Meng, M. Brittnacher, G. Parks, Observation of IMF and seasonal effects in the location of auroral substorm onset. J. Geophys. Res. 106, 5799-5810 (2001). doi:10.1029/2000JA003001

J.A. Lockwood, W.R. Webber, L. Hsieh, Solar flare proton rigidity spectra deduced from cosmic ray neutron monitor observations. J. Geophys. Res. 79, 4149-4155 (1974). doi:10.1029/JA079i028p04149

M. Lockwood, M.J. Owens, L. Barnard, C.J. Davis, F. Steinhilber, The persistence of solar activity indicators and the descent of the Sun into Maunder Minimum conditions. Geophys. Res. Lett. 38, L22105 (2011). doi:10.1029/2011GL049811

Y. Logachev, Catalogues of Solar Proton Events (IZMIRAN, Moscow, 1982)

Y. Logachev, Catalogues of Solar Proton Events (WDC B, Moscow, 1990)

Y. Logachev, Catalogues of Solar Proton Events (Moscow University Press, Moscow, 1998)

M. López-Puertas, B. Funke, S. Gil-López, T. von Clarmann, G.P. Stiller, M. Höpfner, S. Kellmann, G. Mengistu Tsidu, H. Fischer, C.H. Jackman, $\mathrm{HNO}_{3}, \mathrm{~N}_{2} \mathrm{O}_{5}$, and $\mathrm{ClONO}_{2}$ enhancements after the October-November 2003 solar proton events. J. Geophys. Res. 110, A9 (2005). doi:10.1029/2005JA011051

K.R. Lorentzen, M.D. Looper, J.B. Blake, Relativistic electron microbursts during the GEM storms. J. Geophys. Res. 28, 2573-2576 (2001). doi:10.1029/2001GL012926

E.R. Lovejoy, J. Curtius, K.D. Froyd, Atmospheric ion-induced nucleation of sulfuric acid and water. J. Geophys. Res. 109, D8204 (2004). doi:10.1029/2003JD004460

A.T.Y. Lui, W.W. Lai, K. Liou, C.I. Meng, A new technique for short-term forecast of auroral activity. Geophys. Res. Lett. 30, L1258 (2003). doi:10.1029/2002GL016505

L.R. Lyons, Magnetospheric processes leading to precipitation. Space Sci. Rev. 80(1-2), 109132 (1997). doi:10.1023/A:1004977704864

V.S. Makhmutov, G.A. Bazilevskaya, M.B. Krainev, Characteristics of energetic electron precipitation into the earth's polar atmosphere and geomagnetic conditions. Adv. Space Res. 31, 1087-1092 (2003). doi:10.1016/S0273-1177(02)00814-1

V.S. Makhmutov, G.A. Bazilevskaya, Y.I. Stozhkov, A.K. Svirzhevskaya, N.S. Svirzhevsky, Long-Term Balloon Cosmic Ray Experiment:. Results of Analysis of Energetic 
Electron Precipitation Events. Intern-. J. Modern Phys. A 20, 6843-6845 (2005). doi:10.1142/S0217751X05030260

F. Märcz, Short-term changes in atmospheric electricity associated with Forbush decreases. J. Atmos. Solar-Terrest. Phys. 59, 975-982 (1997). doi:10.1016/S1364-6826(96)00076-4

R. Markson, Solar modulation of atmospheric electrification and possible implications for the Sun-weather relationship. Nature 273, 103-109 (1978). doi:10.1038/273103a0

R. Markson, Modulation of the earth's electric field by cosmic radiation. Nature 291, 304-308 (1981). doi:10.1038/291304a0

R. Markson, M. Muir, Solar wind control of the earth's electric field. Science 208, 979-990 (1980). doi:10.1126/science.208.4447.979

D.R. Marsh, R.R. Garcia, D.E. Kinnison, B.A. Boville, F. Sassi, S.C. Solomon, K. Matthes, Modeling the whole atmosphere response to solar cycle changes in radiative and geomagnetic forcing. J. Geophys. Res. 112 D23306 (2007). doi:10.1029/2006JD008306

N. Marsh, H. Svensmark, Cosmic Rays, Clouds, and Climate. Space Sci. Rev. 94, 215-230 (2000)

K.G. McCracken, J. Beer, Long-term changes in the cosmic ray intensity at Earth, 1428-2005. J. Geophys. Res. 112, A10101 (2007). doi:10.1029/2006JA012117

S.B. Mende, H.U. Frey, T.J. Immel, J.-C. Gerard, B. Hubert, S.A. Fuselier, Global Imaging of Proton and Electron Aurorae in the far Ultraviolet. Space Sci. Rev. 109, 211-254 (2003). doi:10.1023/B:SPAC.0000007520.23689.08

N.P. Meredith, R.B. Horne, M.M. Lam, M.H. Denton, J.E. Borovsky, J.C. Green, Energetic electron precipitation during high-speed solar wind stream driven storms. J. Geophys. Res. 116, A5223 (2011). doi:10.1029/2010JA016293

R.A. Mewaldt, C.M.S. Cohen, G.M. Mason, D.K. Haggerty, M.I. Desai, Long-Term Fluences of Solar Energetic Particles from H to Fe. Space Sci. Rev. 130, 323-328 (2007). doi:10.1007/s11214-007-9200-8

R.M. Millan, R.M. Thorne, Review of radiation belt relativistic electron losses. J. Atmos. Sol.-Terr. Phys. 69, 362-377 (2007). doi:10.1016/j.jastp.2006.06.019

R.M. Millan, R.P. Lin, D.M. Smith, K.R. Lorentzen, M.P. McCarthy, X-ray observations of $\mathrm{MeV}$ electron precipitation with a balloon-borne germanium spectrometer. Geophys. Res. Lett. 29, L2194 (2002). doi:10.1029/2002GL015922

R.M. Millan, R.P. Lin, D.M. Smith, M.P. McCarthy, Observation of relativistic electron precipitation during a rapid decrease of trapped relativistic electron flux. Geophys. Res. Lett. 34, 10101 (2007). doi:10.1029/2006GL028653

I.A. Mironova, I.G. Usoskin, Possible effect of extreme solar energetic particle events of September-October 1989 on polar stratospheric aerosols: a case study. Atmos. Chem. Phys. 13, 8543-8550 (2013). doi:10.5194/acp-13-8543-2013

I.A. Mironova, I.G. Usoskin, Possible effect of strong solar energetic particle events on polar stratospheric aerosol: a summary of observational results. Env. Res. Lett. 9(1), 015002 (2014). doi:10.1088/1748-9326/9/1/015002

I.A. Mironova, L. Desorgher, I.G. Usoskin, E.O. Flückiger, R. Bütikofer, Variations of aerosol optical properties during the extreme solar event in January 2005. Geophys. Res. Lett. 35, L18610 (2008). doi:10.1029/2008GL035120

I.A. Mironova, I.G. Usoskin, G.A. Kovaltsov, S.V. Petelina, Possible effect of extreme solar energetic particle event of 20 January 2005 on polar stratospheric aerosols: direct observational evidence. Atmos. Chem. Phys. 12, 769-778 (2012). doi:10.5194/acp-12-769-2012

L.I. Miroshnichenko, J.A. Perez-Peraza, Astrophysical Aspects in the Studies of Solar Cosmic Rays, 2008, pp. 1-141. doi:10.1142/S0217751X08037312

A. Mishev, I. Usoskin, Computations of cosmic ray propagation in the Earth's atmosphere, towards a GLE analysis. Journal of Physics Conference Series 409(1), 012152 (2013). doi:10.1088/1742-6596/409/1/012152

A.L. Mishev, P.I.Y. Velinov, Normalized ionization yield function for various nuclei obtained with full Monte Carlo simulations. Adv. Space Res. 48, 19-24 (2011). doi:10.1016/j.asr.2011.02.008

H. Moraal, A. Belov, J.M. Clem, Design and co-Ordination of Multi-Station International Neutron Monitor Networks. Space Sci. Rev. 93, 285-303 (2000). doi:10.1023/A:1026504814360

H. Moraal, M.S. Potgieter, P.H. Stoker, A.J. van der Walt, Neutron monitor latitude survey of cosmic ray intensity during the 1986/1987 solar minimum. J. Geophys. Res. 94, 1459-1464 (1989). doi:10.1029/JA094iA02p01459

O. Morgenstern, M.A. Giorgetta, K. Shibata, V. Eyring, D.W. Waugh, T.G. Shepherd, H. 
Akiyoshi, J. Austin, A.J.G. Baumgaertner, S. Bekki, P. Braesicke, C. Brühl, M.P. Chipperfield, D. Cugnet, M. Dameris, S. Dhomse, S.M. Frith, H. Garny, A. Gettelman, S.C. Hardiman, M.I. Hegglin, P. Jöckel, D.E. Kinnison, J.-F. Lamarque, E. Mancini, E. Manzini, M. Marchand, M. Michou, T. Nakamura, J.E. Nielsen, D. Olivié, G. Pitari, D.A. Plummer, E. Rozanov, J.F. Scinocca, D. Smale, H. Teyssèdre, M. Toohey, W. Tian, Y. Yamashita, Review of the formulation of present-generation stratospheric chemistryclimate models and associated external forcings. J. Geophys. Res. 115, D00M02 (2010). doi:10.1029/2009JD013728

R.P. Mühleisen, The Global Circuit and Its Parameters (Dietrich Steinkopff Verlag, Darmstadt, 1977), pp. 467-476. ISBN 3798504350

R. Nakamura, N.D. Baker, J.B. Blake, S. Kanekal, B. Klecker, D. Hovestadt, Relativistic electron precipitation enhancements near the outer edge of the radiation belt. Geophys. Res. Lett. 22, 1129-1132 (1995). doi:10.1029/95GL00378

R. Nakamura, M. Isowa, Y. Kamide, D.N. Baker, J.B. Blake, M. Looper, SAMPEX observations of precipitation bursts in the outer radiation belt. Geophys. Res. Lett. 105, 1587515886 (2000). doi:10.1029/2000JA900018

H.V. Neher, Cosmic-Ray Particles That Changed from 1954 to 1958 to 1965. J. Geophys. Res. 72, 1527 (1967). doi:10.1029/JZ072i005p01527

H.V. Neher, Cosmic rays at high latitudes and altitudes covering four solar maxima. J. Geophys. Res. 76, 1637-1651 (1971). doi:10.1029/JA076i007p01637

J. Nevalainen, I.G. Usoskin, A. Mishev, Eccentric dipole approximation of the geomagnetic field: Application to cosmic ray computations. Adv. Space Res. 52, 22-29 (2013). doi:10.1016/j.asr.2013.02.020

P.T. Newell, C.-I. Meng, K.M. Lyons, Suppression of discrete aurorae by sunlight. Nature 381, 766-767 (1996). doi:10.1038/381766a0

P.T. Newell, T. Sotirelis, S. Wing, Seasonal Variations in Diffuse, Monoenergetic, and Broadband Aurora. AGU Fall Meeting Abstracts, 1511 (2009)

P.T. Newell, T. Sotirelis, S. Wing, Seasonal variations in diffuse, monoenergetic, and broadband aurora. J. Geophys. Res. 115, A3216 (2010). doi:10.1029/2009JA014805

P.T. Newell, Y.I. Feldstein, Y.I. Galperin, C.-I. Meng, Morphology of nightside precipitation. J. Geophys. Res. 101, 10737-10748 (1996). doi:10.1029/95JA03516

P.T. Newell, T. Sotirelis, K. Liou, A.R. Lee, S. Wing, J. Green, R. Redmon, Predictive ability of four auroral precipitation models as evaluated using Polar UVI global images. Space Weather 8, 12004 (2010). doi:10.1029/2010SW000604

M. Nicolet, On the production of nitric oxide by cosmic rays in the mesosphere and stratosphere. Planet. Space Sci. 23, 637-649 (1975). doi:10.1016/0032-0633(75)90104-X

K.A. Nicoll, R.G. Harrison, Experimental determination of layer cloud edge charging from cosmic ray ionisation. Geophys. Res. Lett. 37, L13802 (2010). doi:10.1029/2010GL043605

H. Nieder, H. Winkler, D.R. Marsh, M. Sinnhuber, $\mathrm{NO}_{x}$ production due to energetic particle precipitation in the MLT region: Results from ion chemistry model studies. J. Geophys. Res. 119, 2137-2148 (2014). doi:10.1002/2013JA019044

K. O'Brien, Secular variations in the production of cosmogenic isotopes in the earth's atmosphere. J. Geophys. Res. 84, 423-431 (1979)

D.E. Olson, The evidence for auroral effects on atmospheric electricity. Pure Appl.Geophys. 84, 118-138 (1971). doi:10.1007/BF00875461

Y.E. Ozolin, I.L. Karol', E.V. Rozanov, T.A. Egorova, A model of the impact of solar proton events on the ionic and gaseous composition of the mesosphere. Izv. Atmosph. Ocean. Phys. 45, 737-750 (2009). doi:10.1134/S0001433809060073

Particle Data Group, Review of Particle Physics. Phys. Lett. B 592, 228-234 (2004). doi:10.1016/j.physletb.2004.06.001

W.D. Pesnell, R.A. Goldberg, C.H. Jackman, D.L. Chenette, E.E. Gaines, A search of UARS data for ozone depletions caused by the highly relativistic electron precipitation events of May 1992. J. Geophys. Res. 104, 165-176 (1999). doi:10.1029/1998JA900030

W.D. Pesnell, R.A. Goldberg, C.H. Jackman, D.L. Chenette, E.E. Gaines, Variation of mesospheric ozone during the highly relativistic electron event in May 1992 as measured by the High Resolution Doppler Imager instrument on UARS. J. Geophys. Res. 105, 22943-22954 (2000). doi:10.1029/2000JA000091

J.R. Pierce, P.J. Adams, Can cosmic rays affect cloud condensation nuclei by altering new particle formation rates? Geophys. Res. Lett. 36, L9820 (2009). doi:10.1029/2009GL037946

C. Plainaki, A. Belov, E. Eroshenko, H. Mavromichalaki, V. Yanke, Modeling ground 
level enhancements: Event of 20 January 2005. J. Geophys. Res. 112 A4102 (2007). doi:10.1029/2006JA011926

H.S. Porter, C.H. Jackman, A.E.S. Green, Efficiencies for production of atomic nitrogen and oxygen by relativistic proton impact in air. J. Chem. Phys. 65, 154-167 (1976). doi:10.1063/1.432812

M. Potgieter, Solar Modulation of Cosmic Rays. Living Reviews in Solar Physics 10, 3 (2013). doi:10.12942/lrsp-2013-3

G.W. Prölss, Space Weather Effects in the Upper Atmosphere: Low and Middle Latitudes, in Lecture Notes in Physics, Berlin Springer Verlag, ed. by K. Scherer, H. Fichter, B. Herber Lecture Notes in Physics, Berlin Springer Verlag, vol. 656, 2004, p. 193. doi: $10.1007 / \mathrm{b} 100037$

M. Quack, M.-B. Kallenrode, M. von Koenig, K. Kuenzi, J. Burrows, B. Heber, E. Wolff, Ground Level Events and Consequences for Stratospheric Chemistry, in International Cosmic Ray Conference. International Cosmic Ray Conference, vol. 10, 2001, p. 4023

C.E. Randall, V.L. Harvey, C.S. Singleton, S.M. Bailey, P.F. Bernath, M. Codrescu, H. Nakajima, J.M. Russell, Energetic particle precipitation effects on the Southern Hemisphere stratosphere in 1992-2005. J. Geophys. Res. 112, D8308 (2007). doi:10.1029/2006JD007696

J.B. Reagan, R.E. Meyerott, R.W. Nightingale, R.C. Gunton, R.G. Johnson, J.E. Evans, W.L. Imhof, D.F. Heath, A.J. Krueger, Effects of the August 1972 solar particle events on stratospheric ozone. J. Geophys. Res. 86, 1473-1494 (1981). doi:10.1029/JA086iA03p01473

J.B. Reagan, R.E. Meyerott, J.E. Evans, W.L. Imhof, R.G. Joiner, The effects of energetic particle precipitation on the atmospheric electric circuit. J. Geophys. Res. 88, 3869-3878 (1983). doi:10.1029/JC088iC06p03869

T. Reddmann, R. Ruhnke, S. Versick, W. Kouker, Modeling disturbed stratospheric chemistry during solar-induced $\mathrm{NO}_{x}$ enhancements observed with MIPAS/ENVISAT. J. Geophys. Res. 115, 0 (2010). doi:10.1029/2009JD012569

G.D. Reeves, M.G. Taylor, R.H. Friedel, Y. Chen, Relativistic Electron Equatorial Phase Space Densities Gradients from 4 to 20 Re. AGU Fall Meeting Abstracts, 488 (2003)

R. Reiter, Solar flares and their impact on potential gradient and air-earth current characteristics at high mountain stations. Pure Appl. Geophys. 72, 259-267 (1969). doi:10.1007/BF00875709

A.I. Repnev, A.A. Krivolutsky, Variations in the chemical composition of the atmosphere from satellite measurements and their relation to fluxes of energetic particles of cosmic origin (Review). Izv. Atmosph. Ocean. Phys. 46, 535-562 (2010). doi:10.1134/S0001433810050014

J.D. Richardson, J.C. Kasper, A.J. Lazarus, K.I. Paularena, A. Wallace, Solar Cycle Variation of Shocks in the Heliosphere. AGU Fall Meeting Abstracts, 1 (2001)

H. Rishbeth, O.K. Garriott, Introduction to Ionospheric Physics 1969

I.T. Roble R. G., The Global Atmospheric-electrical Circuit (The National Academies Press, Washington DC, 1986), pp. 206-231. ISBN 9780309036801

R.G. Roble, On solar-terrestrial relationships in atmospheric electricity. J. Geophys. Res. 90, 6000-6012 (1985). doi:10.1029/JD090iD04p06000

C.J. Rodger, M.A. Clilverd, D. Nunn, P.T. Verronen, J. Bortnik, E. Turunen, Storm time, short-lived bursts of relativistic electron precipitation detected by subionospheric radio wave propagation. J. Geophys. Res. 112, A7301 (2007). doi:10.1029/2007JA012347

C.J. Rodger, M.A. Clilverd, J.C. Green, M.M. Lam, Use of POES SEM-2 observations to examine radiation belt dynamics and energetic electron precipitation into the atmosphere. J. Geophys. Res. 115, A4202 (2010). doi:10.1029/2008JA014023

B. Rossi, System of Units for Nuclear and Cosmic-Ray Phenomena. Phys. Rev. 57, 660-660 (1940). doi:10.1103/PhysRev.57.660

R. Roussel-Dupré, J.J. Colman, E. Symbalisty, D. Sentman, V.P. Pasko, Physical Processes Related to Discharges in Planetary Atmospheres. Space Sci. Revs. 137, 51-82 (2008). doi:10.1007/s11214-008-9385-5

E. Rozanov, M. Calisto, T. Egorova, T. Peter, W. Schmutz, Influence of the Precipitating Energetic Particles on Atmospheric Chemistry and Climate. Surv. Geophys. 33, 483-501 (2012). doi:10.1007/s10712-012-9192-0

E.V. Rozanov, T.A. Egorova, A.I. Shapiro, W.K. Schmutz, Modeling of the Atmospheric Response to a Strong Decrease of the Solar Activity, in Comparative magnetic minima: characterizing quite times in the Sun and stars, ed. by Mandrini, CH and Webb, DF IAU 
Symposium Proceedings Series, vol. 286, Int Astronom Union; Agencia Nacl Promoc Cientif \& Technol; Consejo Nacl Investigac Cientif \& Tecn; Air Force Off Sci Res, So Off Aerospace Res \& Dev; Sci Comm Solar Terrestrial Phys; Comm Space Res; Comis Nacl Actividades Espaciales; Ctr Latinoamericano Fis, 2012, pp. 215-224. Int Astronom Union; Agencia Nacl Promoc Cientif \& Technol; Consejo Nacl Investigac Cientif \& Tecn; Air Force Off Sci Res, So Off Aerospace Res \& Dev; Sci Comm Solar Terrestrial Phys; Comm Space Res; Comis Nacl Actividades Espaciales; Ctr Latinoamericano Fis. 286th Symposium of the International-Astronomical-Union, Mendoza, Argentina, Oct. 03-07, 2011. ISBN 9781-107-01986-7. doi:10.1017/S1743921312004863

D.W. Rusch, J.-C. Gerard, S. Solomon, P.J. Crutzen, G.C. Reid, The effect of particle precipitation events on the neutral and ion chemistry of the middle atmosphere. I - Odd nitrogen. Planet. Space Sci. 29, 767-774 (1981). doi:10.1016/0032-0633(81)90048-9

M.J. Rycroft, K.A. Nicoll, K.L. Aplin, R.G. Harrison, Recent advances in global electric circuit coupling between the space environment and the troposphere. J. Atmos. Sol.-Terr. Phys., (2012). doi:10.1016/j.jastp.2012.03.015

K. Schlegel, G. Diendorfer, S. Thern, M. Schmidt, Thunderstorms, lightning and solar activityMiddle Europe. J. Atmos. Sol.-Terr. Phys. 63, 1705-1713 (2001). doi:10.1016/S13646826(01)00053-0

L.H. Seeley, G.T. Seidler, J.G. Dash, Laboratory investigation of possible ice nucleation by ionizing radiation in pure water at tropospheric temperatures. J. Geophys. Res. 106, 30333036 (2001). doi:10.1029/2000JD900670

K. Semeniuk, V.I. Fomichev, J.C. McConnell, C. Fu, S.M.L. Melo, I.G. Usoskin, Middle atmosphere response to the solar cycle in irradiance and ionizing particle precipitation. Atmos. Chem. Phys. 11, 5045-5077 (2011). doi:10.5194/acp-11-5045-2011

A. Seppälä, M.A. Clilverd, C.J. Rodger, NOx enhancements in the middle atmosphere during 2003-2004 polar winter: Relative significance of solar proton events and the aurora as a source. J. Geophys. Res. 112, D23303 (2007). doi:10.1029/2006JD008326

A. Seppälä, K. Matthes, C. E. Rondall, I. A. Mironova What is the solar influence on climate? Overview of activities during CAWSES-II. Progress in Earth and Planetary Science. 1, 24 (2014). doi:10.1186/s40645-014-0024-3

V.A. Sergeev, M.V. Malkov, Diagnostics of the magnetic configuration of the plasma sheet according to measurements of energetic electrons above the ionosphere. Geomag. Aeronom. 28, 649-654 (1988)

V.A. Sergeev, G.R. Bikkuzina, P.T. Newell, Dayside isotropic precipitation of energetic protons. Ann. Geophys. 15, 1233-1245 (1997). doi:10.1007/s00585-997-1233-5

V.A. Sergeev, A.G. Iakhnin, R. Pellinen, Mutual location and magnetospheric sources of the penetration of energetic electrons and diffuse and discrete auroras at a preliminary substorm phase. Geomag. Aeronom. 23, 972-978 (1983)

V.A. Sergeev, M. Malkov, K. Mursula, Testing the isotropic boundary algorithm method to evaluate the magnetic field configuration in the tail. J. Geophys. Res. 98, 7609-7620 (1993). doi:10.1029/92JA02587

V.M. Sheftel, O.I. Bandilet, A.N. Yaroshenko, A.K. Chernyshev, Space-time structure and reasons of global, regional, and local variations of atmospheric electricity. J. Geophys. Res. 99, D10797 (1994). doi:10.1029/93JD02857

E.P. Shettle, M.T. DeLand, G.E. Thomas, J.J. Olivero, Long term variations in the frequency of polar mesospheric clouds in the Northern Hemisphere from SBUV. Geophys. Res. Lett. 36, L2803 (2009). doi:10.1029/2008GL036048

J.A. Simpson, The Latitude Dependence of Neutron Densities in the Atmosphere as a Function of Altitude. Phys. Rev. 73, 1389-1391 (1948). doi:10.1103/PhysRev.73.1389

J.A. Simpson, Elemental and Isotopic Composition of the Galactic Cosmic Rays. Annyal Rev. Nuc. Part. Sci. 33, 323-382 (1983). doi:10.1146/annurev.ns.33.120183.001543

J.A. Simpson, A Brief History of Recurrent Solar Modulation of the Galactic Cosmic Rays (1937-1990). Space Sci. Rev. 83, 169-176 (1998)

J.A. Simpson, W. Fonger, S.B. Treiman, Cosmic Radiation Intensity-Time Variations and Their Origin. I. Neutron Intensity Variation Method and Meteorological Factors. Phys. Rev. 90, 934-950 (1953). doi:10.1103/PhysRev.90.934

B.-M. Sinnhuber, P. von der Gathen, M. Sinnhuber, M. Rex, G. König-Langlo, S.J. Oltmans, Large decadal scale changes of polar ozone suggest solar influence. Atmos. Chem. Phys. 6(7), 1835-1841 (2006). doi:10.5194/acp-6-1835-2006.

M. Sinnhuber, H. Nieder, N. Wieters, Energetic Particle Precipitation and the Chem- 
istry of the Mesosphere/Lower Thermosphere. Surv. Geophys. 33, 1281-1334 (2012). doi:10.1007/s10712-012-9201-3

M. Sinnhuber, B. Funke, T. von Clarmann, M. Lopez-Puertas, G.P. Stiller, A. Seppälä, Variability of $\mathrm{NO}_{x}$ in the polar middle atmosphere from October 2003 to March 2004: vertical transport vs. local production by energetic particles. Atmos. Chem. Phys. 14, 7681-7692 (2014). doi:10.5194/acp-14-7681-2014

D.F. Smart, M.A. Shea, E.O. Flückiger, Magnetospheric Models and Trajectory Computations. Space Sci. Rev. 93, 305-333 (2000). doi:10.1023/A:1026556831199

D.F. Smart, M.A. Shea, A.J. Tylka, P.R. Boberg, A geomagnetic cutoff rigidity interpolation tool: Accuracy verification and application to space weather. Adv. Space Res. 37, 12061217 (2006). doi:10.1016/j.asr.2006.02.011

A.K. Smith, R.R. Garcia, D.R. Marsh, J.H. Richter, WACCM simulations of the mean circulation and trace species transport in the winter mesosphere. J. Geophys. Res. 116, D20115 (2011). doi:10.1029/2011JD016083

D.M. Smith, R.P. Lin, K.A. Anderson, K. Hurley, C.M. Johns, High-resolution spectra of 20$300 \mathrm{keV}$ hard X-rays from electron precipitation over Antarctica. J. Geophys. Res. 100, 19675-19686 (1995). doi:10.1029/95JA01472

S. Solomon, P.J. Crutzen, Analysis of the August 1972 solar proton event including chlorine chemistry. J. Geophys. Res. 86, 1140-1146 (1981). doi:10.1029/JC086iC02p01140

S. Solomon, R.G. Roble, P.J. Crutzen, Photochemical coupling between the thermosphere and the lower atmosphere. I - Odd nitrogen from 50 to 120 km. J. Geophys. Res. 87, 7206-7220 (1982). doi:10.1029/JC087iC09p07206

S. Solomon, D.W. Rusch, J.-C. Gerard, G.C. Reid, P.J. Crutzen, The effect of particle precipitation events on the neutral and ion chemistry of the middle atmosphere. II - Odd hydrogen. Planet. Space Sci. 29, 885-893 (1981). doi:10.1016/0032-0633(81)90078-7

T. Stanev, High Energy Cosmic Rays 2010. doi:10.1007/978-3-540-85148-6

Y.I. Stozhkov, N.S. Svirzhevsky, G.A. Bazilevskaya, A.N. Kvashnin, V.S. Makhmutov, A.K. Svirzhevskaya, Long-term (50 years) measurements of cosmic ray fluxes in the atmosphere. Adv. Space Res. 44, 1124-1137 (2009). doi:10.1016/j.asr.2008.10.038

R.D. Strauss, M.S. Potgieter, S.E.S. Ferreira, Modeling ground and space based cosmic ray observations. Adv. Space Res. 49, 392-407 (2012). doi:10.1016/j.asr.2011.10.006

H. Svensmark, T. Bondo, J. Svensmark, Cosmic ray decreases affect atmospheric aerosols and clouds. Geophys. Res. Lett. 361, L15101 (2009). doi:10.1029/2009GL038429

R.M. Thorne, T.P. O'Brien, Y.Y. Shprits, D. Summers, R.B. Horne, Timescale for MeV electron microburst loss during geomagnetic storms. J. Geophys. Res. 110, A9202 (2005). doi:10.1029/2004JA010882

R.M. Thorne, B. Ni, X. Tao, R.B. Horne, N.P. Meredith, Scattering by chorus waves as the dominant cause of diffuse auroral precipitation. Nature 467, 943-946 (2010). doi:10.1038/nature09467

B.A. Tinsley, Influence of Solar Wind on the Global Electric Circuit, and Inferred Effects on Cloud Microphysics, Temperature, and Dynamics in the Troposphere. Space Sci. Rev. 94, $231-258(2000)$

B.A. Tinsley, R.P. Rohrbaugh, M. Hei, K.V. Beard, Effects of Image Charges on the Scavenging of Aerosol Particles by Cloud Droplets and on Droplet Charging and Possible Ice Nucleation Processes. J. Atm. Sci. 57, 2118-2134 (2000)

O.W. Torreson, W.C. Parkinson, O.H. Gish, G.R. Wait, Ocean Atmospheric-electric Results, in Oceanography III: Scientific Results of Cruise VII During 1928-1929 under Command of Captain J.P. Ault (Carnegie Institution of Washington, Researches of the Department of Terrestrial Magnetism)

S.N. Tripathi, S. Vishnoi, S. Kumar, R.G. Harrison, Computationally-efficient Expressions for the Collision Efficiency Between Electrically Charged Aerosol Particles and Cloud Droplets. ArXiv Physics e-prints (2006)

I. Tzur, R.G. Roble, C.C. Reid, H.C. Zhuang, The Response of the Earth's Global Electrical Circuit to a Solar Proton Event, in Weather and Climate Responses to Solar Variations, ed. by B.M. McCormac, 1983, pp. 427-435

I.G. Usoskin, Cosmic rays and climate forcing . Memor. della Soc. Astron. Ital. 82, 937-942 (2011)

I.G. Usoskin, A History of Solar Activity over Millennia. Living Rev. Solar Phys. 10, 1 (2013). doi:10.12942/lrsp-2013-1

I.G. Usoskin, G.A. Kovaltsov, Cosmic ray induced ionization in the atmosphere: 
Full modeling and practical applications. J. Geophys. Res. 111, D21206 (2006). doi:10.1029/2006JD007150

I.G. Usoskin, G.A. Bazilevskaya, G.A. Kovaltsov, Solar modulation parameter for cosmic rays since 1936 reconstructed from ground-based neutron monitors and ionization chambers. J. Geophys. Res. 116, A02104 (2011). doi:10.1029/2010JA016105

I.G. Usoskin, O.G. Gladysheva, G.A. Kovaltsov, Cosmic ray-induced ionization in the atmosphere: spatial and temporal changes. J. Atmos. Solar-Terr. Phys. 66, 1791-1796 (2004). doi:10.1016/j.jastp.2004.07.037

I.G. Usoskin, G.A. Kovaltsov, I.A. Mironova, Cosmic ray induced ionization model CRAC:CRII: An extension to the upper atmosphere. J. Geophys. Res. 115, D10302 (2010). doi:10.1029/2009JD013142

I.G. Usoskin, H. Kananen, K. Mursula, P. Tanskanen, G.A. Kovaltsov, Correlative study of solar activity and cosmic ray intensity. J. Geophys. Res. 103, 9567-9574 (1998). doi:10.1029/97JA03782

I.G. Usoskin, K. Alanko-Huotari, G.A. Kovaltsov, K. Mursula, Heliospheric modulation of cosmic rays: Monthly reconstruction for 1951-2004. J. Geophys. Res. 110, A12108 (2005). doi:10.1029/2005JA011250

I.G. Usoskin, I. Braun, O.G. Gladysheva, J.R. Hörandel, T. Jämsén, G.A. Kovaltsov, S.A. Starodubtsev, Forbush decreases of cosmic rays: Energy dependence of the recovery phase. J. Geophys. Res. 113, A07102 (2008). doi:10.1029/2007JA012955

I.G. Usoskin, L. Desorgher, P. Velinov, M. Storini, E.O. Flückiger, R. Bütikofer, G.A. Kovaltsov, Ionization of the earth's atmosphere by solar and galactic cosmic rays. Acta Geophys. 57, 88-101 (2009). doi:10.2478/s11600-008-0019-9

I.G. Usoskin, G.A. Kovaltsov, I.A. Mironova, A.J. Tylka, W.F. Dietrich, Ionization effect of solar particle GLE events in low and middle atmosphere. Atmos. Chem. Phys. 11, 19791988 (2011). doi:10.5194/acp-11-1979-2011

R. Vainio, L. Desorgher, D. Heynderickx, M. Storini, E. Flückiger, R.B. Horne, G.A. Kovaltsov, K. Kudela, M. Laurenza, S. McKenna-Lawlor, H. Rothkaehl, I.G. Usoskin, Dynamics of the Earth's particle radiation environment. Space Sci. Rev. 147, 187-231 (2009). doi:10.1007/s11214-009-9496-7

P.I. Velinov, L.N. Mateev, Response of the middle atmosphere to Galactic cosmic rays. Geomagn. Aeronom. 30, 593-598 (1990)

P.I.Y. Velinov, S. Asenovski, K. Kudela, J. Lastovicka, L. Mateev, A. Mishev, P. Tonev, Impact of cosmic rays and solar energetic particles on the Earth's ionosphere and atmosphere. J. Space Weather Space Clim. 3(26), 260000 (2013). doi:10.1051/swsc/2013036

P.T. Verronen, R. Lehmann, Analysis and of ionic reactions affecting middle atmospheric $\mathrm{HOx}$ and NOx during proton events. Ann. Geophys. 31, 909-956 (2013). doi:110.5194/angeo31-909-2013

P.T. Verronen, E. Turunen, T. Ulich, E. Kyrölä, Modelling the effects of the October 1989 solar proton event on mesospheric odd nitrogen using a detailed ion and neutral chemistry model. Ann. Geophys. 20, 1967-1976 (2002). doi:10.5194/angeo-20-1967-2002

P.T. Verronen, B. Funke, M. López-Puertas, G.P. Stiller, T. von Clarmann, N. Glatthor, C. Enell, E. Turunen, J. Tamminen, About the increase of $\mathrm{HNO}_{3}$ in the stratopause region during the Halloween 2003 solar proton event. Geophys. Res. Lett. 35, L20809 (2008). doi:10.1029/2008GL035312

P.T. Verronen, C.J. Rodger, M.A. Clilverd, S. Wang, First evidence of mesospheric hydroxyl response to electron precipitation from the radiation belts. J. Geophys. Res. 116, D7307 (2011a). doi:10.1029/2010JD014965

P.T. Verronen, M.L. Santee, G.L. Manney, R. Lehmann, S.-M. Salmi, A. Seppälä, Nitric acid enhancements in the mesosphere during the January 2005 and December 2006 solar proton events. J. Geophys. Res. 116, D17301 (2011b). doi:10.1029/2011JD016075

F.M. Vitt, C.H. Jackman, A comparison of sources of odd nitrogen production from 1974 through 1993 in the Earth's middle atmosphere as calculated using a two-dimensional model. J. Geophys. Res. 101, 6729-6740 (1996). doi:10.1029/95JD03386

T. von Clarmann, N. Glatthor, M. Höpfner, S. Kellmann, R. Ruhnke, G.P. Stiller, H. Fischer, B. Funke, S. Gil-López, M. López-Puertas, Experimental evidence of perturbed odd hydrogen and chlorine chemistry after the October 2003 solar proton events. J. Geophys. Res. 110, A09S45 (2005). doi:10.1029/2005JA011053

C. von Savigny, M. Sinnhuber, H. Bovensmann, J.P. Burrows, M.-B. Kallenrode, M. Schwartz, On the disappearance of noctilucent clouds during the January 2005 solar proton events. 
Geophys. Res. Lett. 34, L2805 (2007). doi:10.1029/2006GL028106

H.D. Voss, W.L. Imhof, M. Walt, J. Mobilia, E.E. Gaines, J.B. Reagan, U.S. Inan, R.A. Helliwell, D.L. Carpenter, J.P. Katsufrakis, Lightning-induced electron precipitation. Nature 312, 740-742 (1984). doi:10.1038/312740a0

H.D. Voss, M. Walt, W.L. Imhof, J. Mobilia, U.S. Inan, Satellite observations of lightning-induced electron precipitation. J. Geophys. Res. 103, 11725-11744 (1998). doi:10.1029/97JA02878

P.K. Wang, S.N. Grover, H.R. Pruppacher, On the Effect of Electric Charges on the Scavenging of Aerosol Particles by Clouds and Small Raindrops. J. Atmos. Sci. 35, 1735-1743 (1978)

Y. Wang, D.J. Jacob, J.A. Logan, Global simulation of tropospheric $\mathrm{O}_{3}-\mathrm{NO}_{x}$-hydrocarbon chemistry 3. Origin of tropospheric ozone and effects of nonmethane hydrocarbons. J. Geophys. Res. 103, 10757-10768 (1998). doi:10.1029/98JD00156

C.T.R. Wilson, Investigation on lightning discharges and on the electric field of thunderstorms. Phil. Trans. Roy. Soc. Lond. A221, 73-115 (1920)

J.R. Winckler, Cosmic-Ray Increase at High Altitude on February 23, 1956. Phys. Rev. 104, 220-220 (1956). doi:10.1103/PhysRev.104.220

S. Wing, P.T. Newell, Central plasma sheet ion properties as inferred from ionospheric observations. J. Geophys. Res. 103, 6785-6800 (1998). doi:10.1029/97JA02994

H. Winkler, S. Kazeminejad, M. Sinnhuber, M.-B. Kallenrode, J. Notholt, Conversion of mesospheric $\mathrm{HCl}$ into active chlorine during the solar proton event in July 2000 in the northern polar region. J. Geophys. Res. 114, 0 (2009). doi:10.1029/2008JD011587

H. Winkler, C. von Savigny, J.P. Burrows, J.M. Wissing, M.J. Schwartz, A. Lambert, M. García-Comas, Impacts of the January 2005 solar particle event on noctilucent clouds and water at the polar summer mesopause. Atmos. Chem. Phys. 12, 5633-5646 (2012). doi:10.5194/acp-12-5633-2012

J.M. Wissing, M.-B. Kallenrode, Atmospheric Ionization Module Osnabrück (AIMOS): A 3-D model to determine atmospheric ionization by energetic charged particles from different populations. J. Geophys. Res. 114, A06104 (2009). doi:10.1029/2008JA013884

G. Witt, The Nature of Noctilucent Clouds., in Space Res. IX, 1969, pp. 157-169

WMO, Scientific Assessment of Ozone Depletion: 2010, Global Ozone Research and Monitoring Project, Report 52, WMO, Geneva, Switzerland, 442 (2011)

F. Yu, Formation of large NAT particles and denitrification in polar stratosphere: possible role of cosmic rays and effect of solar activity. Atmos. Chem. Phys. 4, 2273-2283 (2004)

A.M. Zadorozhnyi, G.A. Tuchkov, V.N. Kikhtenko, J. Lastovicka, J. Boska, A. Novak, Nitric oxide and lower ionosphere quantities during solar particle events of October 1989 after rocket and ground-based measurements. J. Atmos. Sol.-Terr. Phys. 54, 183-192 (1992)

Y. Zhang, L.J. Paxton, An empirical Kp-dependent global auroral model based on TIMED/GUVI FUV data. J. Atmos. Sol.-Terr. Phys. 70, 1231-1242 (2008). doi:10.1016/j.jastp.2008.03.008

H. Ziereis, F. Arnold, Gaseous ammonia and ammonium ions in the free troposphere. Nature 321, 503-505 (1986). doi:10.1038/321503a0 